\title{
EXPERIMENTAL INVESTIGATION OF SECONDARY FLOWS IN A FAMILY OF THREE HIGHLY LOADED LOW-PRESSURE TURBINE CASCADES
}

\author{
by
}

\section{Tatjana Zorić}

A thesis submitted to the Faculty of Graduate Studies and Research in partial fulfillment of the requirements for the degree of

\section{Master of Applied Science}

in Aerospace Engineering

Ottawa-Carleton Institute for Mechanical \& Aerospace Engineering

\author{
Department of Mechanical \& Aerospace Engineering \\ Carleton University \\ Ottawa, Ontario, Canada
}

(c) Tatjana Zorić

April 2006 


$\begin{array}{ll}\begin{array}{l}\text { Library and } \\ \text { Archives Canada }\end{array} & \begin{array}{l}\text { Bibliothèque et } \\ \text { Archives Canada }\end{array} \\ \begin{array}{l}\text { Published Heritage } \\ \text { Branch }\end{array} & \begin{array}{l}\text { Direction du } \\ \text { Patrimoine de l'édition }\end{array} \\ \begin{array}{l}\text { 395 Wellington Street } \\ \text { Ottawa ON K1A ON4 }\end{array} & \begin{array}{l}\text { 395, rue Wellington } \\ \text { Ottawa ON K1A ON4 } \\ \text { Canada }\end{array}\end{array}$

Your file Votre référence ISBN: 978-0-494-16472-3 Our file Notre référence ISBN: 978-0-494-16472-3

NOTICE:

The author has granted a nonexclusive license allowing Library and Archives Canada to reproduce, publish, archive, preserve, conserve, communicate to the public by telecommunication or on the Internet, loan, distribute and sell theses worldwide, for commercial or noncommercial purposes, in microform, paper, electronic and/or any other formats.

The author retains copyright ownership and moral rights in this thesis. Neither the thesis nor substantial extracts from it may be printed or otherwise reproduced without the author's permission.
AVIS:

L'auteur a accordé une licence non exclusive permettant à la Bibliothèque et Archives Canada de reproduire, publier, archiver, sauvegarder, conserver, transmettre au public par télécommunication ou par l'Internet, prêter, distribuer et vendre des thèses partout dans le monde, à des fins commerciales ou autres, sur support microforme, papier, électronique et/ou autres formats.

L'auteur conserve la propriété du droit d'auteur et des droits moraux qui protège cette thèse. $\mathrm{Ni}$ la thèse ni des extraits substantiels de celle-ci ne doivent être imprimés ou autrement reproduits sans son autorisation.
In compliance with the Canadian

Privacy Act some supporting forms may have been removed from this thesis.

While these forms may be included in the document page count, their removal does not represent any loss of content from the thesis.
Conformément à la loi canadienne sur la protection de la vie privée, quelques formulaires secondaires ont été enlevés de cette thèse.

Bien que ces formulaires aient inclus dans la pagination, il n'y aura aucun contenu manquant.

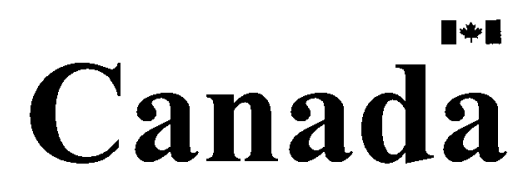


Dedicated to the memory of my dad 


\section{Abstract}

The secondary flows have been investigated in a low speed linear cascade for a family of three low-pressure turbine airfoils designed for the same inlet and outlet flow angles. The baseline airfoil of the family is representative of the midspan section of a vane in a Pratt \& Whitney Aircraft (PWA) engine currently in operation. The other two airfoils, one front- and the other aft-loaded, have $25 \%$ higher loading than the baseline. All three airfoils were designed by PWA.

Measurements were made using a seven-hole pressure probe at a constant Reynolds number of 80,000 (based on the axial chord and the inlet velocity) for three values of incidence, $-5^{\circ}, 0^{\circ}$ (design incidence) and $+5^{\circ}$. The effect of freestream turbulence intensity was also investigated. Two values of turbulence intensity (1.5\% and 4.0\%) were examined at the design and positive incidence. An increase in the freestream turbulence levels resulted in the reduced strength of the secondary flows, and thus the losses.

For all cases examined, the front-loaded airfoil exhibited the highest secondary losses. This was expected based on previous research. The two other airfoils, the baseline and the more aft-loaded one, generated comparable losses. It was suggested that the separation bubbles present on the airfoil surfaces and their interaction with the secondary flows may have been responsible for this unexpected finding. Compared to the empirical loss predictions, a higher sensitivity to the changes in incidence was observed for the airfoils in the present measurements. 


\section{Acknowledgements}

Every journey has its beginning and its end. This thesis marks the end of one such journey, which would never be the same if there were not for a number of people who have left an important trace in my life. Therefore, I would like to take this opportunity to acknowledge at least some of them.

First, I would like to thank my thesis supervisor, Professor Steen Sjolander for his support, advice and guidance. I would also like to express my gratitude and appreciation to Dr. Michael Benner and Ms. Tanya Ellison for their unselfish help. No battle can be won without the comrades in trenches: Mr. Ivan Popović, Dr. Ali Mahallati, Dr. Junqiang Zhu, and Mr. Daniel Knezevici and their support, dedication and patience during numerous sleepless nights and coffee breaks.

There is a number of people who were not directly connected to the project but have often made my life less complicated and more cheerful: Mrs. Nancy Powell, Mrs. Christie Egbert, Mrs. Marlene Groves, Mr. Alex Proctor and Mr. Steve Truttmann.

I would also like to extend my gratitude to Professor Metin Yaras, who many years ago supported my application for transfer to Aerospace Engineering, thus enabling me to pursue my dreams.

Special thanks go to my family: my mother for her unconditional support in all 
my endeavors and my brother for his witty humor which kept me laughing all these years.

Finally, all this would not have been possible without the financial assistance of the Pratt and Whitney Aircraft, which is gratefully acknowledged. 


\section{Table of Contents}

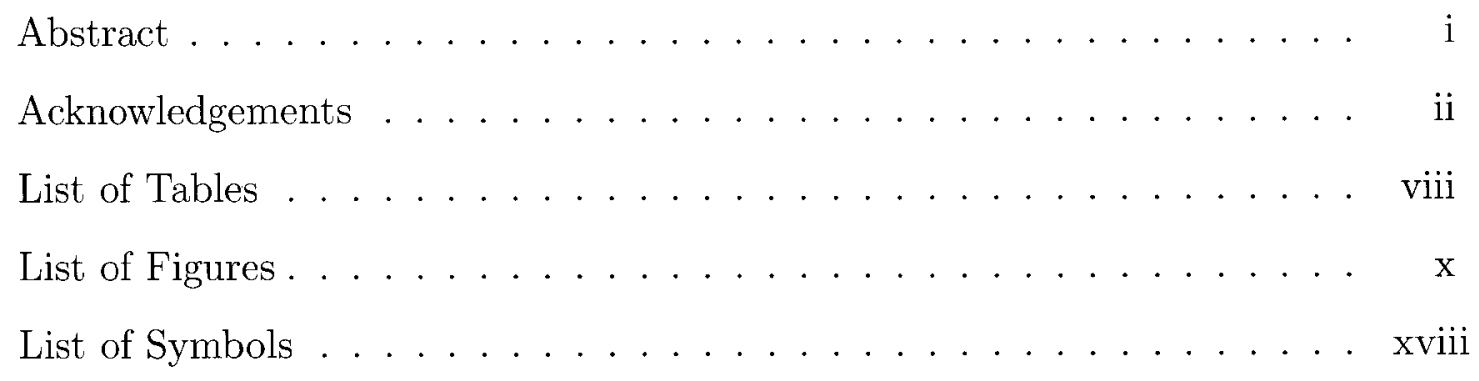

1 Introduction 1

1.1 Motivation ...................... 1

1.2 Thesis Objectives . . . . . . . . . . . . . . 4

1.3 Thesis Overview . . . . . . . . . . . . . . 5

2 Literature Review 6

2.1 Introduction . . . . . . . . . . . . . . . 6

2.2 Nomenclature and Terminology . . . . . . . . . . . . 7

2.2.1 Blade Nomenclature .............. 7

2.2.2 Loss Terminology . . . . . . . . . . . . . 8

2.3 Loss Breakdown Schemes . . . . . . . . . . . . . . . . . . . 10

2.4 Secondary Flows in Turbine Blade Passage . . . . . . . . . . . . . 13

2.5 Experimental Secondary Loss Investigations . . . . . . . . . . 21

2.6 Concluding Remarks . . . . . . . . . . . . . . 26 
3 Experimental Set-up and Measurement Methods $\quad 27$

3.1 Introduction . . . . . . . . . . . . . . 27

3.2 Low-Speed Wind Tunnel . . . . . . . . . . . . . . . . . . . 28

3.3 Test Section . . . . . . . . . . . . . . . . . . 29

3.4 Test Cascades . . . . . . . . . . . . . . . . . . . 31

3.5 Experimental Instrumentation . . . . . . . . . . . . . . . 34

3.5.1 Pressure Transducers . . . . . . . . . . . . . . 34

3.5.2 Temperature Monitor ............... 35

3.5.3 Digital Barometer . . . . . . . . . . . . 35

3.5.4 Data Acquisition System . . . . . . . . . . 36

3.5.5 Probe Calibration Rig ............... 36

3.6 Pressure Probes . . . . . . . . . . . . . . . . . . . . . . 38

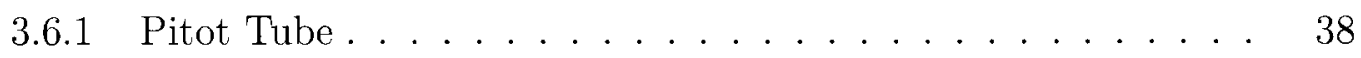

3.6 .2 Three-Hole Probe . . . . . . . . . . . . . . 38

3.6.3 Seven-Hole Probe . . . . . . . . . . . . . . . . 40

3.7 Cascade Testing Procedures . . . . . . . . . . . . . . 41

3.7.1 Wind Tunnel Operating Conditions ............ 41

3.7.2 Inlet Flow Uniformity and Outlet Flow Periodicity . . . . . . 42

3.7.3 Seven-Hole Probe Area Traverses . . . . . . . . . . . . 45

3.8 Data Reduction . . . . . . . . . . . . . . . 47

3.8.1 Measured and Mixed-Out Loss Coefficients . . . . . . . . . 47

3.8 .2 Streamwise Vorticity . . . . . . . . . . . . 49

3.8.3 Axial Velocity Ratio (AVR) ............. 51

4 Secondary Flows at Design Incidence $\quad 53$

4.1 Introduction . . . . . . . . . . . . . . . 53

4.2 Blade Loading . . . . . . . . . . . . . . . 54

V 
4.3 Inlet Endwall Boundary Layer . . . . . . . . . . . . . . . 58

4.4 Detailed Discussion of the Baseline Airfoil . . . . . . . . . . . 62

4.4.1 Total Pressure and Vorticity Distributions . . . . . . . . . 62

4.4.2 Axial Velocity Ratio (AVR) Distribution . . . . . . . . . 64

4.4.3 The Effect of Varying Freestream Turbulence Intensity . . . . 67

4.5 Comparison of Results for All Three Cascades . . . . . . . . . . . 71

4.5.1 Total Pressure and Vorticity Distributions . . . . . . . . 71

4.5.2 Axial Velocity Distribution (AVR) . . . . . . . 77

4.5.3 The Effect of Varying Freestream Turbulence Intensity . . . . 78

4.6 Summary of Results and Conclusions . . . . . . . . . . . . 85

5 Secondary Flows at Off-Design Incidence $\quad 88$

5.1 Introduction . . . . . . . . . . . . . . 88

5.2 Blade Loadings . . . . . . . . . . . . . . . . 89

5.3 Inlet Endwall Boundary Layers . . . . . . . . . . . . . . 93

5.4 Discussion of the Results for the Baseline Cascade . . . . . . . . . . . 94

5.4.1 Total Pressure and Vorticity Distributions . . . . . . . . . . 94

5.4.2 Axial Velocity Distributions (AVR) . . . . . . . . . . . 99

5.4.3 The Effect of Varying FSTI . . . . . . . . . . . . . . . 99

5.5 Comparison of Results for All Three Cascades . . . . . . . . . . . . . 102

5.5.1 Total Pressure and Vorticity Distributions . . . . . . . . . 102

5.5.2 Axial Velocity Ratio (AVR) Distributions . . . . . . . . 109

5.5.3 The Effect of Varying FSTI . . . . . . . . . . . . . . . 109

5.6 Summary of Conclusions . . . . . . . . . . . . . . . 110

6 Comparison of Measured Secondary Losses with Empirical Predictions

6.1 Introduction . . . . . . . . . . . . . . . 114 
6.2 Review of Correlations . . . . . . . . . . . . . . 115

6.3 Evaluation of Correlations . . . . . . . . . . . . . . . . 121

6.3 .1 Introduction . . . . . . . . . . . . . . . . . . 121

6.3.2 New Loss Breakdown Scheme . . . . . . . . . . . . . . . . 121

6.3.3 Evaluation at Design Incidence . . . . . . . . . . . . . 124

6.3.4 Evaluation at Off-Design Incidence . . . . . . . . . . 126

6.4 Concluding Remarks . . . . . . . . . . . . . . . . . 128

7 Conclusions and Recommendations for Future Research $\quad 130$

7.1 Conclusions . . . . . . . . . . . . . . . . . . . . 130

7.2 Recommendations for Future Work . . . . . . . . . . . 135

$\begin{array}{ll}\text { References } & 137\end{array}$

A Calibration Coefficients for Pressure Transducers 147

B Three-Hole Probe Calibration Curves $\quad 149$

C Seven-Hole Probe Calibration Curves 152

D Summary of Experimental Results at Design Incidence 165

E Summary of Experimental Results at Off-Design Incidence 168

F Total Pressure Distribution $\quad 172$

G Positive Vorticity 184

$\begin{array}{ll}\text { H Negative Vorticity } & 196\end{array}$

I Axial Velocity Distribution 208

vii 


\section{List of Tables}

2.1 Summary of Previous Secondary Loss Investigations . . . . . . . . 24

3.1 Summary of Cascade Data . . . . . . . . . . . . . . . . 34

3.2 Pressure Transducers . . . . . . . . . . . . . . . . . 34

4.1 Quality Factor Summary for the Three Cascades . . . . . . . 57

4.2 Inlet Total Pressure Coefficients at Design Incidence for FSTI of $1.5 \%$ and $4.0 \% \ldots \ldots \ldots$. . . . . . . . . . . . . . 61

4.3 Summary of Inlet Boundary Layer Parameters at Design Incidence for FSTI of $1.5 \%$ and $4.0 \% \ldots \ldots 61$

4.4 PAKB: Secondary Losses and Circulation . . . . . . . . . . . . 69

4.5 Comparison of Secondary Losses and Secondary Circulation for the Three Airfoils . . . . . . . . . . . . . . . . . . 76

4.6 Comparison of Secondary Losses and Secondary Circulation for the Three Airfoils at FSTI of $1.5 \%$ and $4.0 \% \ldots \ldots \ldots$

5.1 Summary of the Secondary Loss Measurements . . . . . . . . . 89

5.2 Summary of Integrated Inlet Total Pressure Coefficients at Design and Off-Design Incidence . . . . . . . . . . . . .

5.3 Summary of Inlet Boundary Layer Parameters at Design and OffDesign Incidence . . . . . . . . . . . . . . . . . . . . 94

5.4 PAKB: Secondary Losses and Circulation Variation with Incidence 95 
5.5 PAKB: Secondary Losses and Circulation Variation with FSTI at $+5^{\circ}$ Incidence . . . . . . . . . . . . . . . . 101

5.6 Summary of Mass-Averaged Secondary Losses at Mixed-Out Plane 105

6.1 Summary of Measured and Correlated Secondary Losses for the Three Cascades at Design Incidence . . . . . . . . . . . . . . . . 125

A.1 Pressure Transducers Calibration Coefficients . . . . . . . . . . 148

D.1 Summary of Results at Design Incidence, FSTI $=1.5 \% \ldots \ldots$

D.2 Summary of Results at Design Incidence, FSTI $=4.0 \% \quad \ldots \ldots$

E.1 Summary of Results at $-5^{\circ}$, FSTI $=1.5 \% \ldots \ldots \ldots$

E.2 Summary of Results at $+5^{\circ}$, FSTI $=1.5 \% \ldots \ldots \ldots$

E.3 Summary of Results at $+5^{\circ}$, FSTI $=4.0 \% \ldots \ldots \ldots 171$ 


\section{List of Figures}

1.1 PW4000-112 Engine Cutaway . . . . . . . . . . . . . 3

2.1 Airfoil Geometry Terminology . . . . . . . . . . . . . 8

2.2 Schematic of the Flow Through a Turbine Blade Passage . . . . . 11

2.3 Classification of Flow Regions and Penetration Depth . . . . . . . 12

2.4 Turning of the Endwall Boundary Layer inside the Blade Passage 14

2.5 Secondary Flow Structures According to Hawthorne . . . . . . 16

2.6 Secondary Flow Structures According to Langston et al. . . . . . 16

2.7 Secondary Flow Structures According to Sharma and Butler . . 19

2.8 Secondary Flow Structures According to Wang et al. . . . . . . 19

2.9 Limiting Endwall Flow Patterns . . . . . . . . . . . . . . . . 20

3.1 Carleton University Low-Speed Wind Tunnel . . . . . . . . . . 28

3.2 Carleton University Low-Speed Wind Tunnel Test Section . . . 30

3.3 Grid Mounting Positions and Traverse Planes . . . . . . . . . . . 32

3.4 Airfoil Comparison . . . . . . . . . . . . . . . 33

3.5 Calibration Rig . . . . . . . . . . . . . . . . . . . . 37

3.6 Three-Hole Probe . . . . . . . . . . . . . . . . . . . . . . . 39

3.7 Seven-Hole Probe . . . . . . . . . . . . . . . . . . . . . 40 
3.8 Inlet Flow Uniformity and Outlet Flow Periodicity . . . . . . . . 43

3.9 Inlet Flow Uniformity and Outlet Flow Periodicity . . . . . . . 44

3.10 Measurement Plane . . . . . . . . . . . . . . . . . . . . . 46

3.11 Measurement Matrix . . . . . . . . . . . . . 46

4.1 Blade Loadings at Design Incidence . . . . . . . . . . . . . . 55

4.2 Inlet Velocity Profile - No Grid Installed, FSTI=0.4\% . . . . . 58

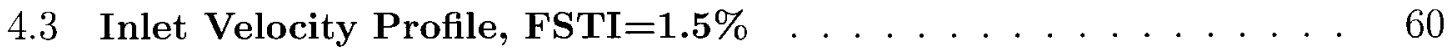

4.4 Inlet Velocity Profile, FSTI=4.0\% f . . . . . . . . . 60

4.5 PAKB - Measurement Plane and Total Pressure Contours at FSTI $=1.5 \% \ldots \ldots \ldots \ldots$. . . . . . . . . . . . 64

4.6 PAKB - Line Contours of Total Pressure Coefficient Superimposed on Flood Contours of Positive Vorticity at FSTI=1.5\% . . . . . . 65

4.7 PAKB - Line Contours of Total Pressure Coefficient Superimposed on Flood Contours of Negative Vorticity at FSTI=1.5\% . . . . 65

4.8 PAKB - Line Contours of Total Pressure Coefficient Superimposed on Flood Contours of Axial Velocity Ratio at FSTI=1.5\% . . . . 66

4.9 PAKB - Total Pressure Contours at FSTI=1.5\% and 4.0\% . . . 68

4.10 PAKB - Line Contours of Total Pressure Coefficient Superimposed on Flood Contours of Positive Vorticity at FSTI $=1.5 \%$ and $4.0 \%$

4.11 PAKB - Line Contours of Total Pressure Coefficient Superimposed on Flood Contours of Negative Vorticity at FSTI $=1.5 \%$ and $4.0 \% \quad 70$

4.12 Total Pressure Loss Contours - Comparison of the Three Airfoils

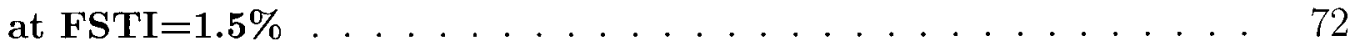

4.13 Positive Vorticity - Comparison of the Three Airfoils at FSTI=1.5\% 74

4.14 Negative Vorticity - Comparison of the Three Airfoils at FSTI=1.5\% 75

4.15 AVR Contours - Comparison of the Three Airfoils at FSTI=1.5\% 79

4.16 Total Pressure Loss Contours - Comparison of the Three Airfoils at $\mathrm{FSTI}=4.0 \% \ldots \ldots \ldots$. . . . . . . . . . . . . . 81

4.17 Positive Vorticity - Comparison of the Three Airfoils at FSTI=4.0\% 82 
4.18 Negative Vorticity - Comparison of the Three Airfoils at FSTI=4.0\% 83

4.19 AVR Contours - Comparison of the Three Airfoils at FSTI=4.0\% 86

5.1 Blade Loadings at Off-Design Incidence at FSTI=1.5\% . . . . . . 91

5.2 Blade Loadings at Off-Design Incidence at FSTI $=1.5 \%$ and $\mathbf{4 \%} \quad . \quad 92$

5.3 PAKB: Total Pressure Loss Contours at Design and Off-Design Incidence at $\mathrm{FSTI}=1.5 \% \ldots \ldots . \ldots 96$

5.4 PAKB: Positive Vorticity at Design and Off-Design Incidence at

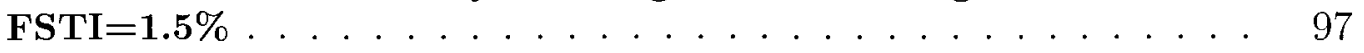

5.5 PAKB: Negative Vorticity at Design and Off-Design Incidence at FSTI $=1.5 \% \ldots \ldots \ldots . \ldots \ldots$

5.6 PAKB: AVR Distribution at Design and Off-Design Incidence at FSTI $=1.5 \% \ldots \ldots \ldots \ldots 10 \ldots$

5.7 PAKB: Total Pressure Contours at $+5^{\circ}$ Incidence and FSTI of $1.5 \%$ and $4.0 \% \ldots \ldots \ldots$. . . . . . . . . . . . . . . 102

5.8 PAKB: Positive Vorticity at $+5^{\circ}$ Incidence and FSTI of $1.5 \%$ and $4.0 \% \ldots \ldots \ldots \ldots$. . . . . . . . . . . . . . . . . . . . . .

5.9 PAKB: Negative Vorticity at $+5^{\circ}$ Incidence and FSTI of $1.5 \%$ and 4.0\% . . . . . . . . . . . . . . . . 103

5.10 Mass-Averaged Secondary Losses at Mixed-Out Plane . . . . . . 104

5.11 PAKD-A: Total Pressure Loss Contours at Design and Off-Design Incidence . . . . . . . . . . . . . . . 106

5.12 PAKD-A: Positive Vorticity Variation With Incidence at FSTI of $1.5 \% \ldots \ldots \ldots \ldots$. . . . . . . . . . . . . . . . . . . . . . .

5.13 PAKD-A : Negative Vorticity Variation With Incidence at FSTI of $1.5 \% \ldots \ldots \ldots \ldots \ldots$

5.14 PAKD-A: Total Pressure Loss Contours at $+5^{\circ}$ Incidence and FSTI of $1.5 \%$ and $4.0 \% \ldots \ldots \ldots 110$

5.15 PAKD-A: Positive Vorticity Variation With FSTI at $+5^{\circ}$ Incidence 111

5.16 PAKD-A : Negative Vorticity Variation With FSTI at $+\mathbf{5}^{\circ}$ Incidence 11 
6.1 Comparison of Secondary Losses for the Three Cascades Using Conventional and New Loss Breakdown Schemes at FSTI=1.5\%

6.2 Comparison of Secondary Losses for the Three Cascades Using Conventional and New Loss Breakdown Schemes at FSTI=4.0\%

6.3 Evaluation of Correlation due to Moustapha et al. (1990) . . . . 127

6.4 Evaluation of Correlation due to Benner et al. (2005b) . . . . . 127

A.1 Sample Calibration Curves . . . . . . . . . . . . . . . . . 148

B.1 Three-Hole Probe Calibration Curves . . . . . . . . . . . . . . 151

B.2 Three-Hole Probe Calibration Curves . . . . . . . . . . . 151

C.1 $C_{\alpha}$ Tangential Coordinate System for Low-Angle Regime . . . . . 154

C.2 $C_{\alpha}$ Seven-Hole Probe Numbering Scheme . . . . . . . . . . 154

C.3 $C_{\alpha}$ Calibration Data for Sector $7 \ldots \ldots \ldots \ldots$

C.4 $C_{\beta}$ Calibration Data for Sector $7 \ldots \ldots \ldots \ldots$

C.5 $C_{0}$ Calibration Data for Sector $7 \ldots \ldots \ldots \ldots$

C.6 $C_{Q}$ Calibration Data for Sector $7 \ldots \ldots \ldots$

C.7 $C_{\alpha}$ Polar Coordinates System Used for High-Angle Regime . . . . 160

C.8 $C_{\alpha}$ Division of Angular Space . . . . . . . . . . . . . . 160

C.9 $C_{\phi}$ Calibration Data for Sector $4 \ldots \ldots \ldots 1$

C.10 $C_{\theta}$ Calibration Data for Sector $4 \ldots \ldots \ldots 2$

C.11 $C_{0}$ Calibration Data for Sector $4 \ldots \ldots \ldots 3$

C.12 $C_{Q}$ Calibration Data for Sector $4 \ldots \ldots \ldots$. . . . . . 164

F.1 PAKB: Total Pressure Contours at Design and Off-Design Incidence at FSTI $=1.5 \% \ldots \ldots \ldots \ldots$. . . . . . . . . . . . . . . . . . . .

F.2 PAKB: Total Pressure Contours at Design Incidence at FSTI $=1.5 \%$

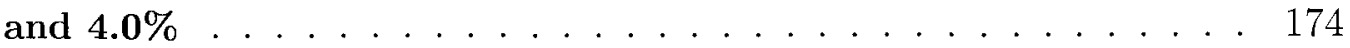


F.3 PAKB: Total Pressure Contours at $+5^{\circ}$ Incidence at FSTI $=1.5 \%$ and $4.0 \% \ldots \ldots \ldots \ldots \ldots \ldots$

F.4 PAKD-A: Total Pressure Contours at Design and Off-Design Incidence at FSTI $=1.5 \% \ldots \ldots \ldots$. . . . . . . . . . 175

F.5 PAKD-A: Total Pressure Contours at Design Incidence at FSTI $=1.5 \%$ and $4.0 \% \ldots \ldots \ldots \ldots$. . . . . . . . . . . . . . . . 176

F.6 PAKD-A: Total Pressure Contours at $+5^{\circ}$ Incidence at FSTI $=1.5 \%$ and $4.0 \% \ldots \ldots \ldots \ldots$. . . . . . . . . . . . . . . 176

F.7 PAKD-F: Total Pressure Contours at Design and Off-Design Incidence at FSTI=1.5\% . . . . . . . . . . . . . . 177

F.8 PAKD-F: Total Pressure Contours at Design Incidence at FSTI $=1.5 \%$

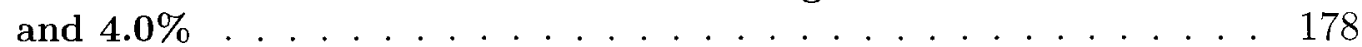

F.9 PAKD-F: Total Pressure Contours at $+5^{\circ}$ Incidence at FSTI $=1.5 \%$

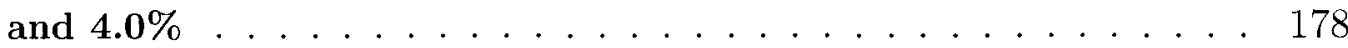

F.10 Total Pressure Contours at $-5^{\circ}$ Incidence at FSTI=1.5\% . . . . . 179

F.11 Total Pressure Contours at $0^{\circ}$ Incidence at FSTI=1.5\% . . . . . 180

F.12 Total Pressure Contours at $+5^{\circ}$ Incidence at FSTI=1.5\% . . . . . 181

F.13 Total Pressure Contours at $0^{\circ}$ Incidence at FSTI=4.0\% . . . . . . 182

F.14 Total Pressure Contours at $+5^{\circ}$ Incidence at FSTI=4.0\% $\ldots \ldots .183$

G.1 PAKB - Line Contours of Total Pressure Coefficient Superimposed on Flood Contours of Positive Vorticity at Design and Off-Design Conditions at FSTI $=1.5 \%$

G.2 PAKB - Line Contours of Total Pressure Coefficient Superimposed on Flood Contours of Positive Vorticity at Design Incidence at FSTI $=1.5 \%$ and $4.0 \% \ldots \ldots \ldots \ldots$

G.3 PAKB - Line Contours of Total Pressure Coefficient Superimposed on Flood Contours of Positive Vorticity at $+5^{\circ}$ Incidence at FSTI $=1.5 \%$ and $4.0 \%$. . . . . . . . .

G.4 PAKDA - Line Contours of Total Pressure Coefficient Superimposed on Flood Contours of Positive Vorticity at Design and OffDesign Conditions at FSTI $=1.5 \%$ 
G.5 PAKDA - Line Contours of Total Pressure Coefficient Superimposed on Flood Contours of Positive Vorticity at Design Incidence at $\mathrm{FSTI}=1.5 \%$ and $4.0 \% \ldots \ldots \ldots$

G.6 PAKDA - Line Contours of Total Pressure Coefficient Superimposed on Flood Contours of Positive Vorticity at $+5^{\circ}$ Incidence at FSTI $=1.5 \%$ and $4.0 \% \ldots \ldots \ldots$

G.7 PAKDF - Line Contours of Total Pressure Coefficient Superimposed on Flood Contours of Positive Vorticity at Design and OffDesign Conditions at FSTI $=1.5 \% \ldots \ldots . . . \ldots$

G.8 PAKDF - Line Contours of Total Pressure Coefficient Superimposed on Flood Contours of Positive Vorticity at Design Incidence at $\mathrm{FSTI}=1.5 \%$ and $4.0 \% \ldots \ldots \ldots$

G.9 PAKDF - Line Contours of Total Pressure Coefficient Superimposed on Flood Contours of Positive Vorticity at $+5^{\circ}$ Incidence at FSTI $=1.5 \%$ and $4.0 \% \ldots \ldots \ldots$

G.10 Line Contours of Total Pressure Coefficient Superimposed on Flood Contours of Positive Vorticity at $-5^{\circ}$ Incidence at FSTI $=1.5 \% 191$

G.11 Line Contours of Total Pressure Coefficient Superimposed on Flood Contours of Positive Vorticity at $0^{\circ}$ Incidence at FSTI $=1.5 \% 192$

G.12 Line Contours of Total Pressure Coefficient Superimposed on Flood Contours of Positive Vorticity at $+5^{\circ}$ Incidence at FSTI $=1.5 \% 193$

G.13 Line Contours of Total Pressure Coefficient Superimposed on Flood Contours of Positive Vorticity at $0^{\circ}$ Incidence at FSTI $=4.0 \% 194$

G.14 Line Contours of Total Pressure Coefficient Superimposed on Flood Contours of Positive Vorticity at $+5^{\circ}$ Incidence at FSTI $=4.0 \% 195$

H.1 PAKB - Line Contours of Total Pressure Coefficient Superimposed on Flood Contours of Negative Vorticity at Design and Off-Design Conditions at FSTI $=1.5 \% \ldots \ldots \ldots$

H.2 PAKB - Line Contours of Total Pressure Coefficient Superimposed on Flood Contours of Negative Vorticity at Design Incidence at FSTI $=1.5 \%$ and $4.0 \% \ldots \ldots \ldots$. . . . . . . . . . . . . . . .

H.3 PAKB - Line Contours of Total Pressure Coefficient Superimposed on Flood Contours of Negative Vorticity at $+5^{\circ}$ Incidence at FSTI $=1.5 \%$ and $4.0 \%$ 
H.4 PAKDA - Line Contours of Total Pressure Coefficient Superimposed on Flood Contours of Negative Vorticity at Design and Off-Design Conditions at FSTI $=1.5 \% \ldots \ldots \ldots$

H.5 PAKDA - Line Contours of Total Pressure Coefficient Superimposed on Flood Contours of Negative Vorticity at Design Incidence at FSTI $=1.5 \%$ and $4.0 \%$

H.6 PAKDA - Line Contours of Total Pressure Coefficient Superimposed on Flood Contours of Negative Vorticity at $+5^{\circ}$ Incidence at $\mathrm{FSTI}=1.5 \%$ and $4.0 \% \ldots \ldots$

H.7 PAKDF - Line Contours of Total Pressure Coefficient Superimposed on Flood Contours of Negative Vorticity at Design and Off-Design Conditions at FSTI $=1.5 \% \ldots \ldots \ldots$

H.8 PAKDF - Line Contours of Total Pressure Coefficient Superimposed on Flood Contours of Negative Vorticity at Design Incidence at FSTI $=1.5 \%$ and $4.0 \%$

H.9 PAKDF - Line Contours of Total Pressure Coefficient Superimposed on Flood Contours of Negative Vorticity at $+5^{\circ}$ Incidence at $\mathrm{FSTI}=1.5 \%$ and $4.0 \% \ldots \ldots \ldots 202$

H.10 Line Contours of Total Pressure Coefficient Superimposed on Flood Contours of Negative Vorticity at $-5^{\circ}$ Incidence at FSTI $=\mathbf{1 . 5 \%} 203$

H.11 Line Contours of Total Pressure Coefficient Superimposed on Flood Contours of Negative Vorticity at $0^{\circ}$ Incidence at FSTI $=1.5 \% 204$

H.12 Line Contours of Total Pressure Coefficient Superimposed on Flood Contours of Negative Vorticity at $+5^{\circ}$ Incidence at FSTI $=\mathbf{1 . 5} \% 205$

H.13 Line Contours of Total Pressure Coefficient Superimposed on Flood Contours of Negative Vorticity at $0^{\circ}$ Incidence at FSTI $=4.0 \% 206$

H.14 Line Contours of Total Pressure Coefficient Superimposed on Flood Contours of Negative Vorticity at $+5^{\circ}$ Incidence at FSTI $=\mathbf{4 . 0} \% 207$

I.1 PAKB - Line Contours of Total Pressure Coefficient Superimposed on Flood Contours of AVR at Design and Off-Design Conditions at $\mathrm{FSTI}=1.5 \% \ldots \ldots \ldots \ldots$. . . . . . . . . . . . . . . . . . . . . .

I.2 PAKB - Line Contours of Total Pressure Coefficient Superimposed on Flood Contours of AVR at Design Incidence at FSTI $=1.5 \%$ and $4.0 \%$ 
I.3 PAKB - Line Contours of Total Pressure Coefficient Superimposed on Flood Contours of AVR at $+5^{\circ}$ Incidence at FSTI $=1.5 \%$ and $4.0 \% \ldots \ldots \ldots \ldots \ldots \ldots$

I.4 PAKDA - Line Contours of Total Pressure Coefficient Superimposed on Flood Contours of AVR at Design and Off-Design Conditions at FSTI $=1.5 \%$

I.5 PAKDA - Line Contours of Total Pressure Coefficient Superimposed on Flood Contours of AVR at Design Incidence at FSTI $=1.5 \%$ and $4.0 \% \ldots \ldots \ldots \ldots$

I.6 PAKDA - Line Contours of Total Pressure Coefficient Superimposed on Flood Contours of AVR at $+5^{\circ}$ Incidence at FSTI $=1.5 \%$ and $4.0 \% \ldots \ldots \ldots \ldots \ldots$

I.7 PAKDF - Line Contours of Total Pressure Coefficient Superimposed on Flood Contours of AVR at Design and Off-Design Conditions at FSTI $=1.5 \%$

I.8 PAKDF - Line Contours of Total Pressure Coefficient Superimposed on Flood Contours of AVR at Design Incidence at FSTI $=1.5 \%$ and $4.0 \% \ldots \ldots \ldots . \ldots \ldots 214$

I.9 PAKDF - Line Contours of Total Pressure Coefficient Superimposed on Flood Contours of AVR at $+5^{\circ}$ Incidence at FSTI $=1.5 \%$ and $4.0 \% \ldots \ldots \ldots \ldots \ldots . \ldots \ldots . \ldots \ldots$

I.10 Line Contours of Total Pressure Coefficient Superimposed on Flood Contours of AVR at $-5^{\circ}$ Incidence at FSTI $=1.5 \%$. . . . 215

I.11 Line Contours of Total Pressure Coefficient Superimposed on Flood Contours of AVR at $0^{\circ}$ Incidence at FSTI=1.5\% . . . . . . 216

I.12 Line Contours of Total Pressure Coefficient Superimposed on Flood Contours of AVR at $+5^{\circ}$ Incidence at FSTI=1.5\% . . . . 217

I.13 Line Contours of Total Pressure Coefficient Superimposed on Flood Contours of AVR at $0^{\circ}$ Incidence at FSTI=4.0\% . . . . . . 218

I.14 Line Contours of Total Pressure Coefficient Superimposed on Flood Contours of AVR at $+5^{\circ}$ Incidence at FSTI $=4.0 \% \ldots$ 


\section{List of Symbols}

$\begin{array}{ll}\text { A } & \text { Area } \\ \text { c } & \text { Axial Velocity Ratio defined in Section } 3.8 .3 \\ C_{L} & \text { Blade chord length } \\ C_{P l} & \text { Lift coefficient based on vector mean velocity defined in Section } 6.2 \\ C_{P 0} & \text { Total pressure coefficient } \\ C_{P S} & \text { Static pressure coefficient } \\ C_{Q} & \text { Dynamic pressure coefficient } \\ C_{Q W T} & \text { Wind tunnel calibration coefficient defined in Equation } 3.3 \\ \text { CR } & \text { Convergence ratio defined in Section } 6.2 \\ C_{S K E} & \text { Non-dimensional secondary kinetic energy coefficient defined in Equation } 3.17 \\ C_{\Gamma_{s}} & \text { Non-dimensional secondary circulation defined in Equation } 3.15 \\ C_{\omega_{s}} & \text { Non-dimensional streamwise vorticity defined in Equation } 3.13 \\ \mathrm{~d} & \text { Leading edge diameter } \\ \mathrm{E} & \text { Transducer voltage } \\ \mathrm{h} & \text { Blade span } \\ H_{S F} & \text { Shape factor }=\delta^{*} / \theta \\ i & \text { Inlet flow incidence }=\alpha+\alpha_{1 \text { des }}\end{array}$

xviii 


\begin{tabular}{|c|c|}
\hline M & Mach number \\
\hline o & Throat opening \\
\hline $\mathrm{P}$ & Pressure \\
\hline $\mathrm{R}$ & Radius of curvature \\
\hline SKE & Secondary Kinetic Energy defined in Equation 3.16 \\
\hline$q$ & Dynamic pressure $=\frac{1}{2} \rho V^{2}$ \\
\hline $\mathrm{Q}$ & Quality factor defined in Equations 4.1 \\
\hline $\operatorname{Re}$ & Reynolds number $=\frac{\rho V_{1} C_{x}}{\mu}$ \\
\hline s & Pitch (spacing) \\
\hline$s^{*}$ & Cross-passage distance between suction and pressure surface at $X_{m} i n$ \\
\hline t & Thickness \\
\hline$T_{u}$ & Turbulence intensity \\
\hline$u, v, w$ & Velocity components in axial, pitchwise and spanwise directions, respectively \\
\hline$v_{S E C}, w_{S E C}$ & Components of secondary velocity vector \\
\hline $\mathrm{V}$ & Velocity (absolute) \\
\hline$X_{\min }$ & Axial location of the suction peak measured from the leading edge \\
\hline y & Tangential (pitchwise) coordinate \\
\hline $\mathrm{Y}$ & Pressure loss coefficient defined in Equation 2.2 \\
\hline $\mathrm{z}$ & Spanwise coordinate \\
\hline$Z_{T E}$ & Penetration depth at trailing edge defined in Section 2.2 .2 \\
\hline$Z_{w}$ & Zweifel coefficient \\
\hline
\end{tabular}

\section{Greek}

$\alpha$ 


$\begin{array}{ll}\delta & \text { Boundary layer thickness } \\ \delta^{*} & \text { Boundary layer displacement thickness }=\int_{0}^{\delta}\left(1-\frac{V}{V_{B L}}\right) d z \\ \Delta & \text { Delta (difference) } \\ \varepsilon & \text { Gas (flow) turning in radians } \\ \theta & \text { Boundary layer momentum thickness }=\int_{0}^{\delta}\left(1-\frac{V}{V_{B L}}\right) \frac{V}{V_{B L}} d z \\ \kappa & \text { Specific heat ratio } \\ \mu & \text { Dynamic viscosity } \\ \rho & \text { Density } \\ \phi & \text { Kinetic energy coefficient defined in Equation } 2.3 \\ \sigma & \text { Solidity }=\frac{C}{s} \\ \omega & \text { Vorticity }\end{array}$

\section{Subscripts}

$\begin{array}{ll}0 & \text { Total (stagnation) conditions } \\ 1 & \text { Inlet } \\ 2 & \text { Outlet } \\ \text { BL } & \text { Boundary layer } \\ \mathrm{c} & \text { Chord } \\ \text { des } & \text { Design } \\ \text { ideal } & \text { Ideal conditions } \\ \text { is } & \text { Isentropic } \\ 1 & \text { Local } \\ \text { m } & \text { Mixed-out } \\ \text { max } & \text { Maximum } \\ \text { mean } & \text { Mean (i.e. vector mean angle } \\ \text { min } & \text { Minimum }\end{array}$




$\begin{array}{ll}\text { P } & \text { Profile (i.e. loss) } \\ \text { ref } & \text { Reference conditions } \\ \text { S } & \text { Static conditions } \\ \text { s } & \text { Streamwise (i.e. vorticity component) } \\ \text { SEC } & \text { Secondary (i.e. loss) } \\ \text { TIP } & \text { Tip (i.e. tip-leakage loss) } \\ \text { TOT } & \text { Total (i.e. loss) } \\ \text { x } & \text { Axial } \\ \text { y } & \text { Tangential (pitchwise) }\end{array}$

\section{$\underline{\text { Superscripts }}$}

Mass-averaged in pitchwise direction (at midspan)
" $\quad$ Mass-averaged in both pitchwise and spanwise directions

\begin{tabular}{ll}
\multicolumn{2}{l}{ Abbreviations } \\
3HP & Three-Hole Probe \\
$5 \mathrm{HP}$ & Five-Hole Probe \\
$7 \mathrm{HP}$ & Seven-Hole Probe \\
AC & Alternating Current (i.e. electric motor \\
AR & Aspect Ratio \\
BP & Blade passage \\
CL & Center Line \\
EW & End Wall \\
EWP & End Wall Profiling \\
FC & Flow control \\
FSTI & Free Stream Turbulence Intensity
\end{tabular}


$\mathrm{HF}$

Hot Film

HP Horse Power

HSV Horse Shoe Vortex

HW Hot Wire

IBL Inlet Boundary Layer

LE Leading Edge

LP Low-Pressure

LPT Low-Pressure Turbine

LSTL Low-Speed Turbomachinery Laboratory

PS Pressure Side (Surface)

SS Suction Side (Surface)

TE Trailing Edge

XW Cross (hot) Wire 


\section{Chapter 1}

\section{Introduction}

\subsection{Motivation}

The enormous growth in air travel and stringent safety and environmental concerns have pushed the aircraft manufacturers to design and build new airplanes that will adhere to the regulations while maintaining their operational cost effectiveness in order to remain competitive on the market. Many of the improvements in aircraft economics are planned to come directly from improved engine performance. This is creating additional pressure on the already fierce competition between engine manufactures for the design of more efficient and cost-effective engines. This in itself is not an easy task as the engine component efficiencies are already so high that design limits are being reached and significant jumps in performance are becoming virtually impossible to achieve.

For this reason, a lot of effort in turbomachinery is currently being directed towards the design of highly loaded blades. The utilization of these blades in 
turbomachines involves increasing the pitch-to-chord ratio between adjacent blades resulting in a reduction in the number of blades and thus increasing aerodynamic loading on the remaining airfoils. Highly loaded blades, which essentially offer the same performance with component weight reduction, will directly result in significant cost savings.

This particular area of research is pushing the envelope of the traditional design guidelines and consequently a lot of effort is being invested into research that will shed more light on the behavior of the highly loaded blades, expose the problems associated with their employment in the real engine environment and offer the solutions to the problems encountered. Much of this effort is directed towards weight reduction of the low-pressure turbine (LPT).

The LPT drives the low-pressure compressor and the fan in high bypass turbofan engines such as the PW4000 series engine shown in Figure 1.1. These engines are used to power twin-jet wide-body airplanes such as the Boeing B777 and Airbus A330. As much as $80 \%$ of the thrust in a high-bypass engine is delivered by the fan. To be able to provide the needed power, LPTs typically consist of a number of stages (seven LPT stages in PW4000), and may contain up to one thousand blades. This makes the low-pressure turbine one of the heaviest engine components, and the weight of the LPT may account for as much as $1 / 3$ of the total engine weight (Howell et al., 2001).

The low-pressure turbine typically employs long blades with high aspect ratios. As a result, most of the flow over the LPT vane is essentially two-dimensional. This two-dimensional flow over the blades is generally referred to as the primary flow. Secondary flows, on the other hand, are the three-dimensional flows that arise in the regions close to the endwalls. They are formed when the inlet endwall boundary layer is turned through an angle by a curved passage (i.e. adjacent blades). As a 


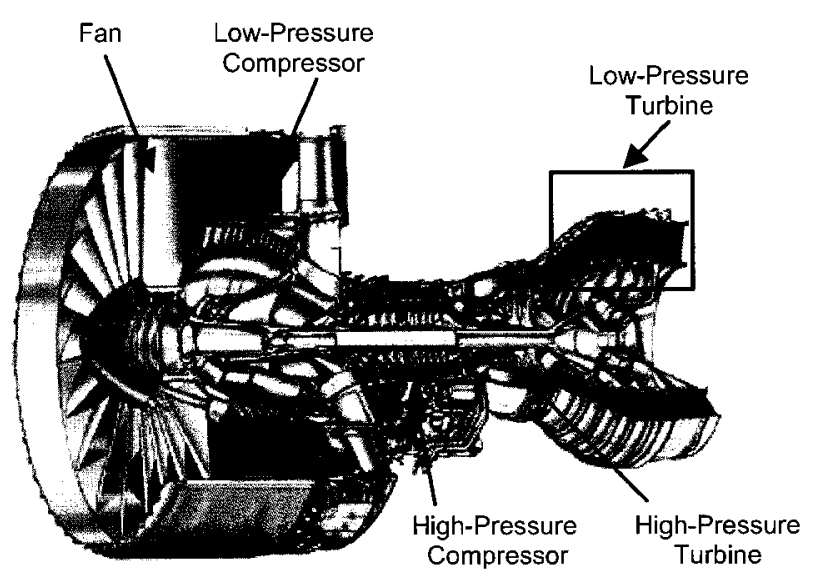

Figure 1.1: PW4000-112 Engine Cutaway

result of the turning and the cross-passage pressure gradient, a streamwise vorticity is generated whose mixing represents a major source of loss. Although not predominant, the secondary losses can still account for about $1 / 3$ of the total pressure loss in lowpressure turbines.

During its typical operation, an aircraft engine is subjected to a variety of conditions ranging from take-off to cruise at high altitude. Thus, for a significant portion of their operational life the engine components may be working at off-design conditions. Operation at off-design conditions may result in increase in component losses, which in turn, would decrease the engine efficiency. It is therefore important to investigate the effects of off-design operation on losses. Consequently, a significant part of the present research was devoted to investigation of performance at off-design conditions.

The present work is a part of comprehensive ongoing project sponsored by Pratt \& Whitney Aircraft (US). The present investigation examines the behavior of a family of three highly loaded low-pressure turbine airfoils. In the past couple of years, 
several researchers have examined the primary flow (the flow at midspan of the blades) for these three airfoils under various steady and unsteady aerodynamic conditions. Hence, the study by the present author focused on the flows in the blade endwall regions (secondary flows) and losses for the same cascades.

\subsection{Thesis Objectives}

Secondary losses in turbine blade rows have been the topic of many investigations over the past decades, but up to this point, to the author's knowledge, no studies have looked at the secondary flows and losses associated with them for highly loaded low-pressure turbine blades.

As previously mentioned, the objective of this thesis is to investigate the secondary losses in a family of three highly loaded low-pressure turbine airfoils. All three airfoils were designed by Pratt \& Whitney Aircraft. The airfoil denoted as PAKB represents the baseline design of this family, and is representative of the midspan section of an LPT vane of recent design. The two additional airfoils, designated PAKD, are derivatives of the baseline blade. They are $25 \%$ more loaded than the baseline blade, and the main difference between the two PAKD airfoils is in their loading distributions. The PAKD-A airfoil is aft-loaded (similar to the baseline), while the PAKD-F is forward-loaded.

The thesis investigates the influence of the level of loading and its distribution on the secondary flows. In addition, the effect of the freestream turbulence intensity (FSTI) on the secondary flows is also examined by employing turbulence generating grids that generate FSTIs of about $1.5 \%$ and $4.0 \%$. All measurements were performed in a low-speed linear cascade at a constant Reynolds number of 80,000 based on axial 
chord and inlet velocity. Three values of incidence were examined: design $\left(0^{\circ}\right)$ and two values of off-design incidence $\left(-5^{\circ}\right.$ and $\left.+5^{\circ}\right)$.

\subsection{Thesis Overview}

Chapter 2 reviews the literature most relevant to the present investigation. The following chapter provides an overview of the experimental apparatus, measurement procedures and data reduction methods. The results of the secondary flow measurements at design and off-design incidence are discussed in Chapters 4 and 5, respectively. Comparison of the measured results with empirical predictions at design and off-design conditions is presented in Chapter 6. Finally the conclusions of the present investigation and recommendations for future work are given in Chapter 7 . 


\section{Chapter 2}

\section{Literature Review}

\subsection{Introduction}

This chapter gives an overview of the literature related to past investigations and background information regarding secondary flows in low-pressure turbines in the following fashion. Firstly the nomenclature and the terminology used will be introduced. Next, an overview of the conventional loss breakdown in axial flow turbines will be given. A description of secondary flows in turbine blade passage will be given in Section 2.4, while Section 2.5 will provide a summary of the previous secondary flow investigations. Finally, the chapter will close with some concluding remarks. 


\subsection{Nomenclature and Terminology}

\subsubsection{Blade Nomenclature}

The definition of incidence is introduced in Figure 2.1. For the purposes of this study the incidence is defined as the difference between the actual $\left(\alpha_{1}\right)$ and the design inlet flow angle $\left(\alpha_{1 \text { des }}\right)$ and is given by equation 2.1:

$$
i=\alpha_{1}-\alpha_{1 d e s}
$$

The design flow angle, as the name suggests, is the inlet flow angle for which the airfoil is designed. If the flow approaches the airfoil at a flow angle different from its design value it is said that the blade is at incidence $(i)$, which can be either positive or negative. Such conditions will be referred to as off-design conditions for the remainder of this thesis.

Figure 2.1 presents the airfoil terminology used. The parameters are defined below. It should be noted that all angles are measured relative to the axial direction.

$$
\begin{array}{ll}
\alpha_{1}: \text { Inlet flow angle } & C: \text { True chord } \\
\alpha_{1 \text { des }}: \text { Inlet design flow angle } & C_{x} \text { : Axial chord } \\
\alpha_{2}: \text { Outlet flow angle } & i \text { : Incidence } \\
\beta_{1}: \text { Inlet metal angle } & o: \text { Throat opening } \\
\beta_{2}: \text { Outlet metal angle } & s: \text { Pitch (spacing) } \\
\gamma: \text { Stagger angle } & t_{T E} \text { : Trailing edge thickness }
\end{array}
$$




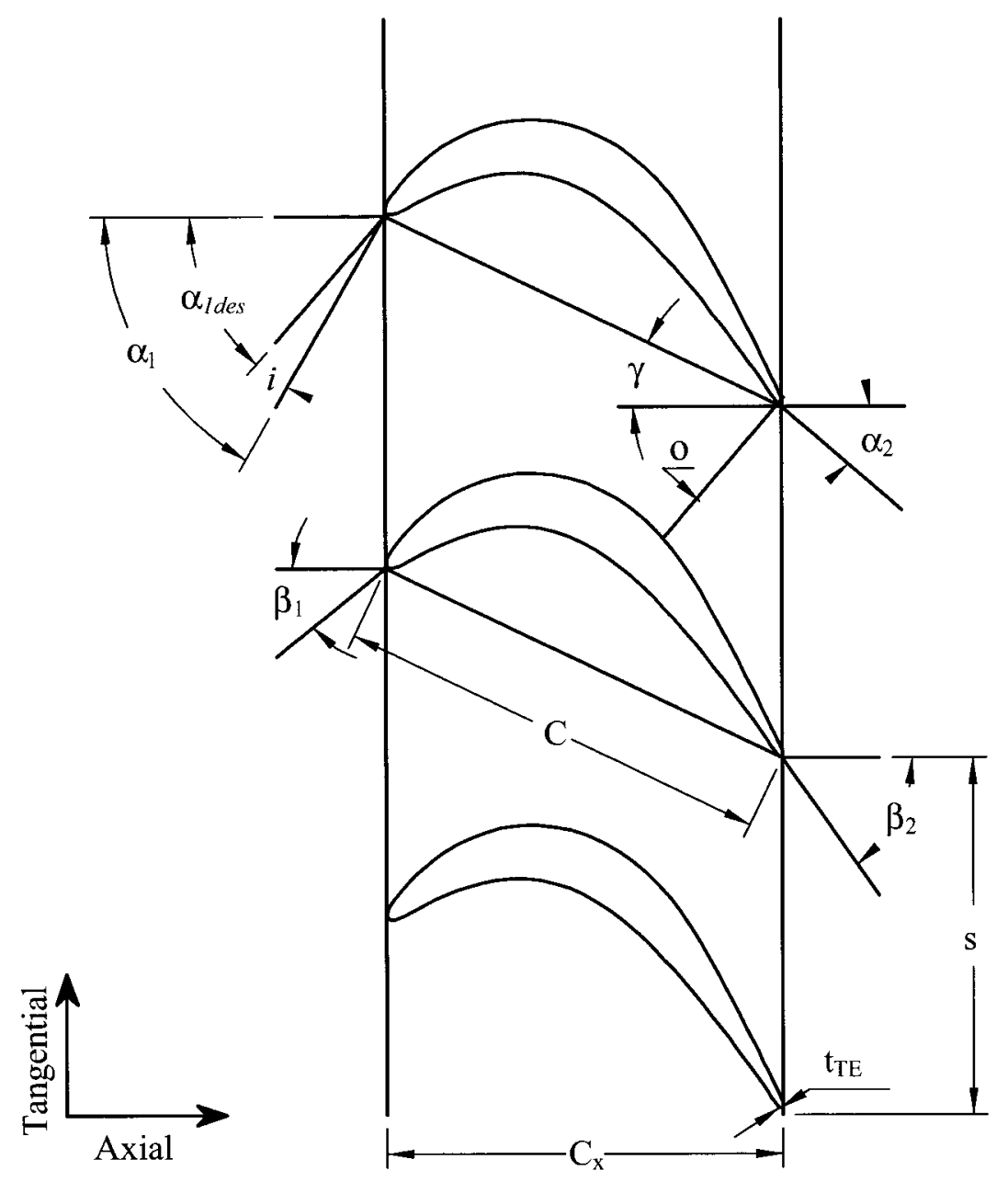

Figure 2.1: Airfoil Geometry Terminology

\subsubsection{Loss Terminology}

Denton (1993) defines loss as any flow feature that reduces the efficiency of a turbomachine. Most turbomachines operate at nearly adiabatic conditions, thus thermodynamic irreversibilities are considered to be the main contributors to the loss of efficiency. These irreversibilities produce entropy. However, the quantification 
of entropy is very difficult as it cannot be measured or seen directly, but can only be inferred from measurements of other properties. Thus, the losses in turbomachines are usually expressed in terms of total pressure loss coefficient, $\mathrm{Y}$. In turbines, $\mathrm{Y}$ is defined as:

$$
Y=\frac{P_{01}^{\prime \prime}-P_{02}^{\prime \prime}}{q_{2}^{\prime \prime}}
$$

where $P_{0}^{\prime \prime}$ and $q^{\prime \prime}$ are the mass-averaged total and dynamic pressures respectively, and subscripts 1 and 2 denote inlet and outlet conditions.

Alternatively, the loss can be expressed in terms of the mass-averaged kinetic energy coefficient, $\phi^{2}$ which is given by:

$$
\phi^{2}=\frac{\left(V_{2}^{2}\right)^{\prime \prime}}{\left(V_{2 i s}^{2}\right)^{\prime \prime}}
$$

where $V_{2}$ and $V_{2 i s}$ are the actual and isentropic velocity at the outlet, respectively. The isentropic velocity is obtained from isentropic expansion to the same outlet static pressure as that of the actual process. $\phi^{2}$ and $Y$ are related through:

$$
Y=\frac{\left[1-\frac{\kappa-1}{2} M_{2}^{2}\left(\frac{1}{\phi^{2}}-1\right)\right]^{-\frac{\kappa}{\kappa-1}}-1}{1-\left(1+\frac{\kappa-1}{2} M_{2}^{2}\right)^{-\frac{\kappa}{\kappa-1}}}
$$

where $M_{2}$ represents the Mach number at exit, and $\kappa$ is the ratio of specific heats ( $\kappa=1.4$ for air). In incompressible flow, as is the case in this investigation, equation 2.4 reduces to: 


$$
Y=\frac{1}{\phi^{2}}-1
$$

The losses given in equations 2.2, and 2.3 represent the total losses. The total losses can be further broken down into several individual components. The loss breakdown will be discussed in the following section.

\subsection{Loss Breakdown Schemes}

Typically the losses present in a turbomachinery blade passage are divided into the following components:

- Profile Losses, $Y_{P}$

- Tip Leakage Losses, $Y_{T I P}$

- Secondary Losses, $Y_{S E C}$

The losses are considered to be independent of one another so that the total losses, $Y_{T O T}$, are given by:

$$
Y_{T O T}=Y_{P}+Y_{T I P}+Y_{S E C}
$$

Profile Losses are the two-dimensional losses generated in the blade boundary layers, away from the endwalls. The additional loss that arises at the blade trailing edge is usually included in the profile losses. Profile losses are also sometimes called "primary" losses. 
Tip Leakage Losses are associated with the leakage flow between the blade tip and the annulus wall in rotors as can be seen in Figure 2.2. The tip leakage losses were not investigated and will not be discussed further.

Secondary Losses are also known as the "endwall losses" and they refer to the losses arising on the annulus walls in and out of the blade passage as well as the losses associated with mixing of the vortical structures present in the blade passage (Figure 2.2). The secondary losses are the main topic of this investigation and will be discussed in greater detail throughout this thesis. Figure 2.3 presents a view of the suction surface of a blade showing the primary and secondary flow regions as they appear on the blade surface. The width of the secondary flow regions at the

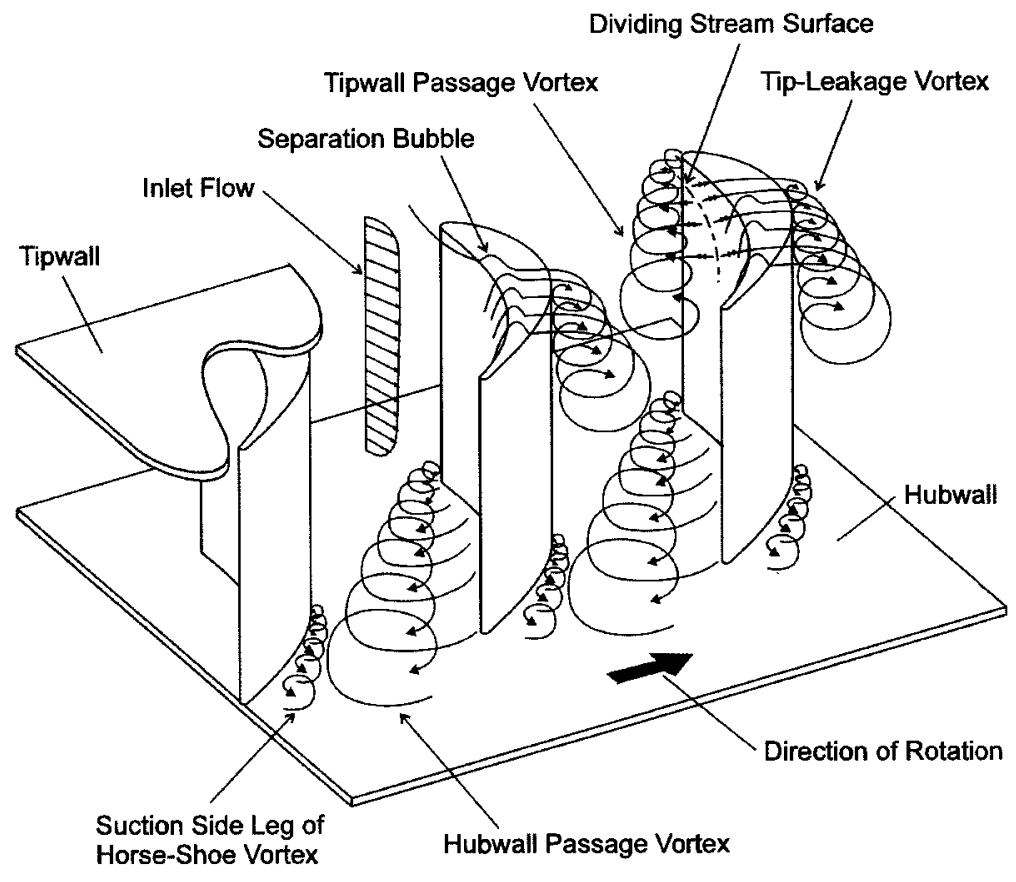

Figure 2.2: Schematic of the Flow Through a Turbine Blade Passage (reproduced from Jeffries, 2001) 
blade trailing edge is denoted by a parameter called penetration depth or height, $Z_{T E}$. The penetration depth was first discussed by Sharma and Butler (1987) and a new correlation for the penetration depth was recently proposed by Benner et al. (2005a).

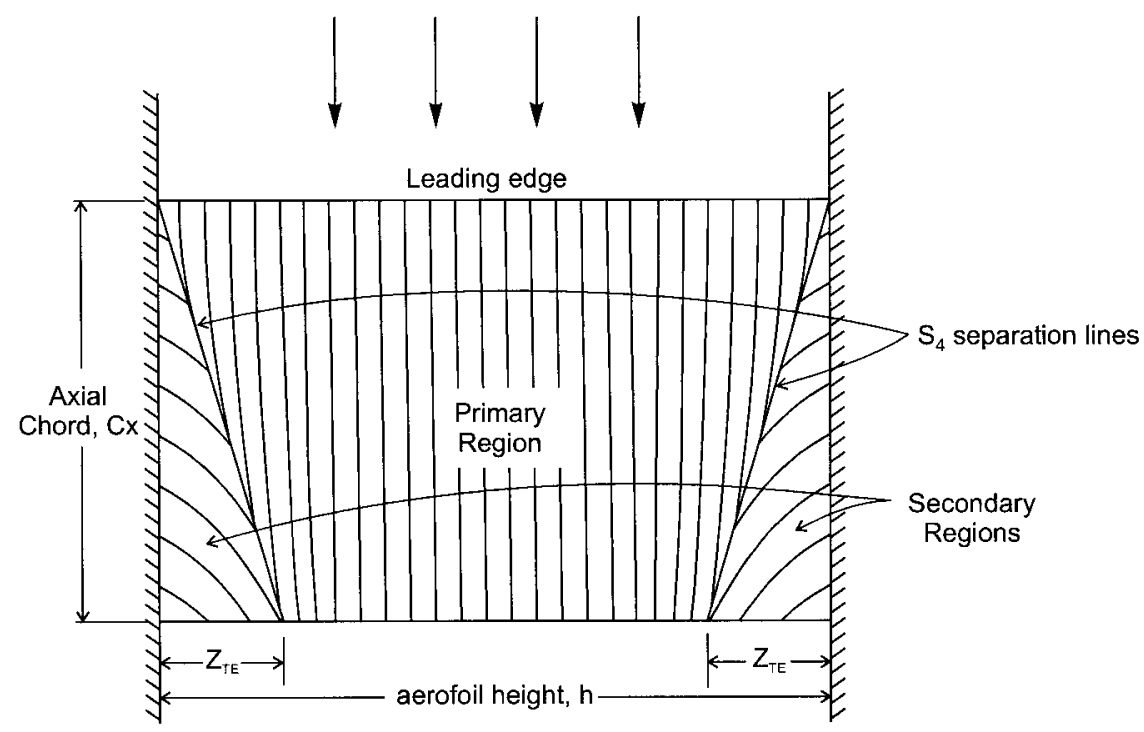

Figure 2.3: Classification of Flow Regions and Penetration Depth (reproduced from Benner, 2003)

Benner et al. (2005a) are of the opinion that loss coefficients obtained from the conventional decomposition can be physically misleading. The main shortcoming of the classical loss breakdown scheme lies in the assumption that the loss generated on the blade suction surface is the same in the primary and secondary regions and is given by the profile loss coefficient, $Y_{P}$. This assumption is particularly unsatisfactory at large values of positive incidence at which the boundary layer in the primary region is typically separated, resulting in high values of the profile loss. Under these conditions, the boundary layers in the secondary region are normally still attached and using the profile loss coefficient significantly overestimates the losses. Benner et al. $(2005 \mathrm{a}, \mathrm{b})$ therefore proposed an alternative breakdown of the losses. The new 
loss decomposition in the absence of the tip leakage is given in equation 2.7:

$$
Y_{T O T A L}=Y_{P}+Y_{S E C}=Y_{M I D S P A N}\left(1-\frac{Z_{T E}}{h}\right)+Y_{S E C}
$$

where $Z_{T E}$ is the penetration depth and $\mathrm{h}$ is the blade height. In the early stages of turbine design, the profile losses would be obtained from design or off-design loss correlations such as Kacker and Okapuu (1982) or Benner et al. (1997), respectively. The new loss breakdown will be further discussed in Section 6.3.2.

\subsection{Secondary Flows in Turbine Blade Passage}

As previously mentioned, the secondary flows are three-dimensional flows found at the hub of the rotor blades and the hub and the shroud of the stator blades. They are formed when the inlet endwall boundary layer is turned through an angle by an adjacent curved surface. A transverse pressure gradient, shown in Figure 2.4, acting in the direction from the blade pressure surface to the suction surface of the adjacent blade exists within the blade passage.

This transverse pressure gradient is defined in equation 2.8 as:

$$
\frac{\partial P_{S}}{\partial R}=\frac{\rho V^{2}}{R}
$$

where $P_{S}$ is the static pressure, $\mathrm{R}$ is the radius of curvature, $\mathrm{V}$ is the velocity and $\rho$ is the density. Both the inlet endwall boundary layer and the freestream flow in the passage are subjected to the same pressure gradient. However, since the velocity in the endwall boundary layer is lower, its streamlines consequently have smaller radius 


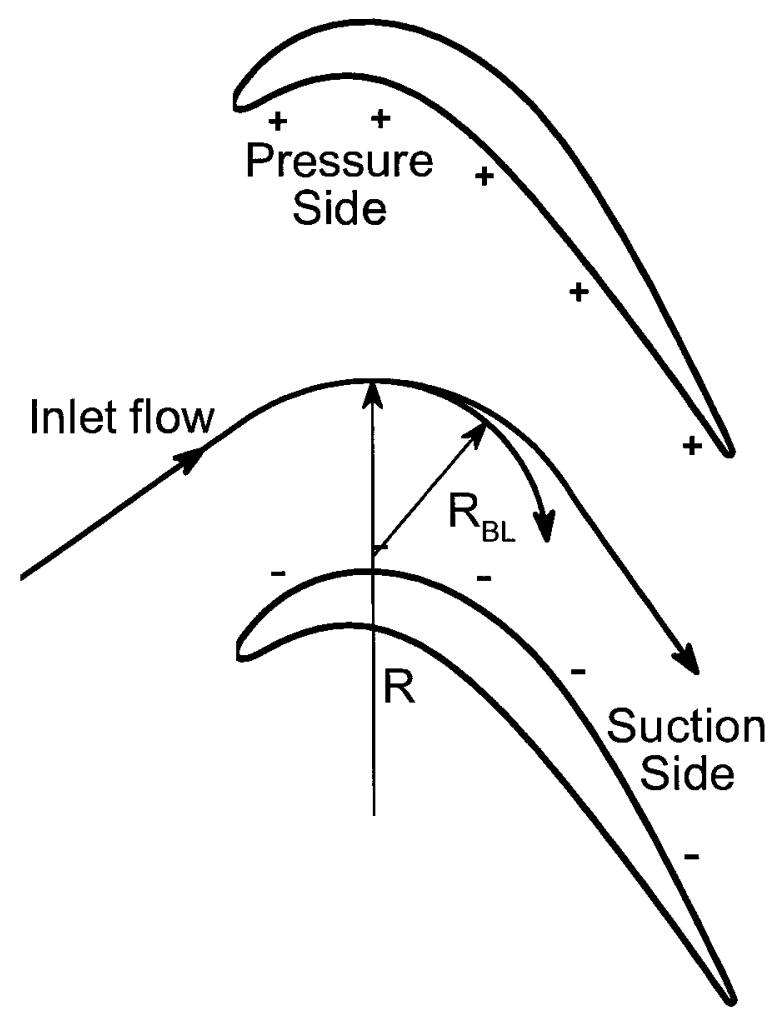

Figure 2.4: Turning of the Endwall Boundary Layer inside the Blade Passage

of curvature than that of the freestream flow. As a result the fluid in the boundary layer is swept towards the suction surface as it turns through the blade passage. Eventually it hits the suction surface and then it moves over the suction surface in the spanwise direction away from the endwall, thus giving rise to a vortical structure. Although the secondary flows are created from the inlet boundary layer fluid, they are an inviscid phenomena. The losses due to secondary losses arise from the mixing of the vortices, their interaction with boundary layers and separation bubbles, if present on the blade surface.

Contrary to what the name "secondary" would suggest, these flows may have a great impact on the overall turbine efficiency. The fraction of the secondary loss in 
the total aerodynamic loss varies from one blade row to another. Thus various sources report different secondary loss fractions. For example, Denton (1993) indicates that it is not uncommon that the secondary losses in turbines can account for as much as $1 / 3$ of the total pressure loss.

Secondary flows have been the topic of many studies in the past decades. Consequently, the physical understanding of the secondary flows has evolved from the one of the first models of Hawthorne (1955) to the more complex models, such as those due to Sharma and Butler (1987) and Wang et al. (1997). The aim of this section is to provide a brief historical overview of the evolution in understanding of the secondary flow structure and physics.

The classical model describing the secondary flows offered by Hawthorne (1955) is shown Figure 2.5. At the passage inlet, the vorticity vector is perpendicular to the flow. By the time the flow from the inlet plane reaches the outlet, the vorticity axis is twisted owing to the distortion of filaments of the inlet boundary layer due to flow passing through a curved passage. As a result, three components of secondary circulation are present downstream of the blade. The first is the distributed secondary vorticity which forms within the blade passage. This distributed secondary vorticity manifests itself as the passage vortices. The second component is the trailing filament vortices that arise from the stretching of the inlet vortex filaments due to velocity gradients between the pressure and the suction surfaces, and the last is the trailing shed vorticity which arises due to spanwise changes of blade circulation.

Using flow visualization Langston et al. (1977) were able to gain a better understanding of the passage flow physics and behavior and proposed a new description of the secondary flows (shown in Figure 2.6). Their model was able to capture the evolution of the vortex through the blade passage. They concluded that the inlet 


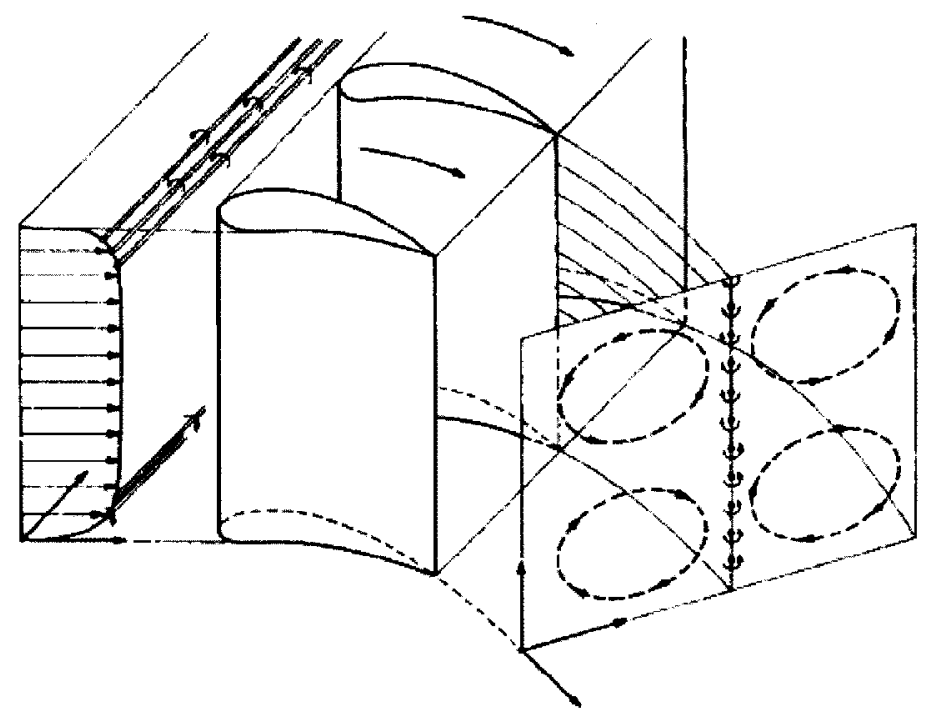

Figure 2.5: Secondary Flow Structures According to Hawthorne (reproduced from Hawthorne, 1955)

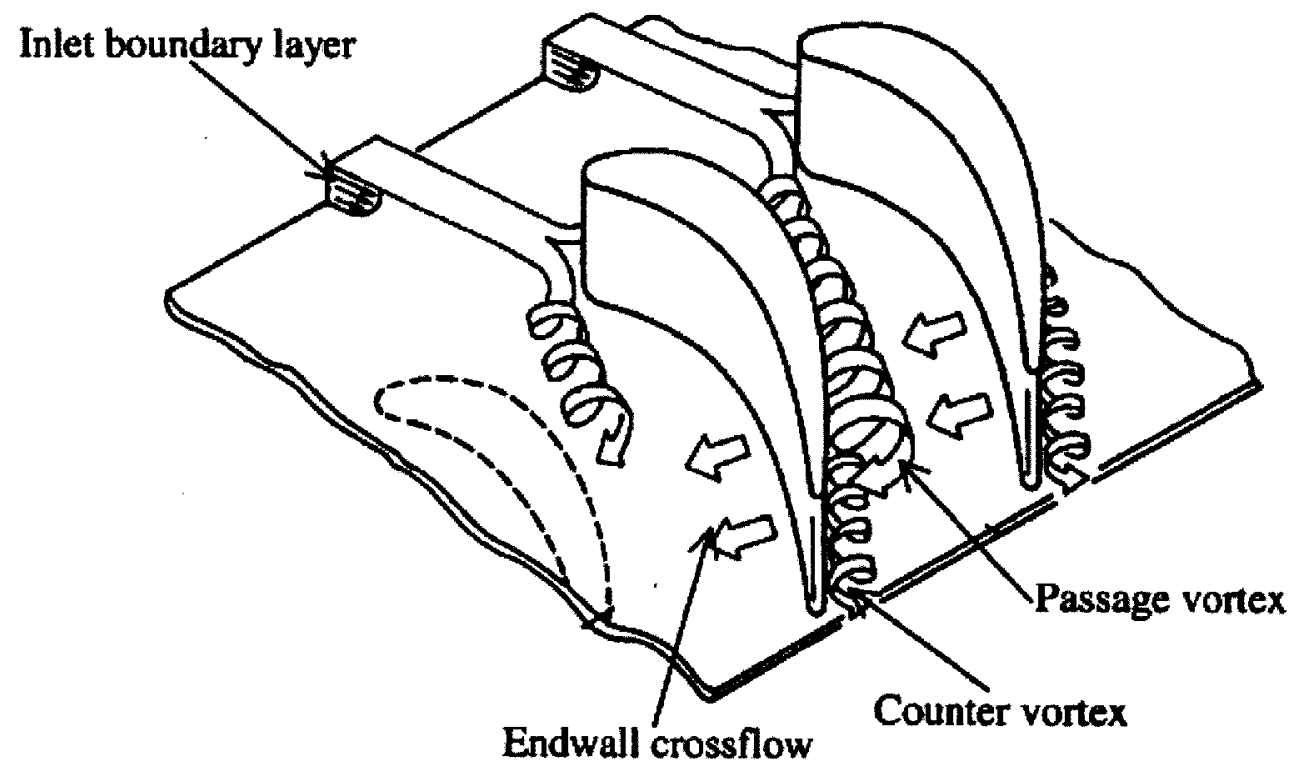

Figure 2.6: Secondary Flow Structures According to Langston et al. (reproduced from Langston et al., 1977) 
boundary layer separates on the endwall at a saddle point ahead of the leading edge. This results in a horseshoe vortex with "pressure" side and "suction" legs. The two legs of the vortex have the opposite sense of rotation. The smaller, suction side leg of the horseshoe vortex is also known as the counter vortex. The pressure side leg of the vortex is fed by the pressure-to-suction surface endwall flow to create what is known as the passage vortex.

Sieverding and den Bosche (1983) confirmed Langston's et al. findings with one difference: in their case, the counter vortex was located on the midspan side of the passage vortex and not on the endwall side, as reported by Langston et al. Sieverding and den Bosche also believed that the suction side of the horseshoe vortex wrapped itself around the pressure side vortex.

More recently Sharma and Butler (1987) proposed a new secondary flow model similar to that of Sieverding and den Bosche, but with an addition of the third vortex structure, a suction side vortex that follows the airfoil contour closely until it gets entrained by the passage vortex. The third vortical structure forms from the inlet boundary layer closest to the endwall. This new vortex is then convected along the suction side moving spanwise to the top edge of the passage vortex, as seen in Figure 2.7. Sharma and Butler also provided a new insight into the effect of the inlet boundary layer on secondary flows. The classical secondary flow theory relies on the assumption that the inlet boundary layer entering the cascade experiences the flow turning in the blade passage resulting in distortion of vortex tubes, thus leading to an increase in streamwise vorticity. Sharma and Butler disagree with this statement based on their review of flow measurements and visualization conducted in the vicinity of the leading edge of the airfoil. They believe that the majority of the fluid from the inlet boundary layer is entrained by the leading edge horseshoe vortex and that 
the normal component of the vorticity associated with the inlet boundary layer is transformed into the streamwise component of vorticity as soon as the fluid enters the cascade: that is, much before the most of the turning is under way. In this way all of the fluid from the inlet boundary layer either become a part of the suction side leg of the horseshoe vortex or is deflected by the pressure-to-suction surface pressure gradient to climb the suction surface on top of the passage vortex. The new boundary layer that forms on the endwalls is entrained by the pressure side of the horseshoe vortex to become a part of the much larger passage vortex.

Wang et al. (1997) extended on the previous work and did extensive research on secondary flows using visual aids (laser lights and multiple smoke wires). Their findings shed new light on the flow features present in turbomachinery blade passages. More specifically, they proposed the existence of a periodically varying multi vortex pattern just upstream of a blade leading edge. This pattern is shown in cross-section AA in Figure 2.8. Their findings confirmed those of Sharma and Butler that the pressure leg of the horseshoe vortex merges with and becomes part of a much larger passage vortex, with the suction leg wrapping itself about the passage vortex. Wang et al. were the first to mention the so called "wall" vortex, which is induced by the passage vortex and remains located close to the suction surface and above the passage vortex. It is believed that the wall vortex is formed by the inlet boundary layer that originates in the area between the saddle points ahead of the cascade.

More recently, Benner (2003) has investigated the physics of the secondary flows in a linear cascade of turbine blades at both design and off-design incidence. Figure 2.9 shows the endwall flow patterns identified by Benner (2003).

The two separation lines, $S_{1}$ and $S_{2}$, upstream of the blade leading edge are caused by the endwall inlet boundary layer separation ahead of the horseshoe vortex and by 


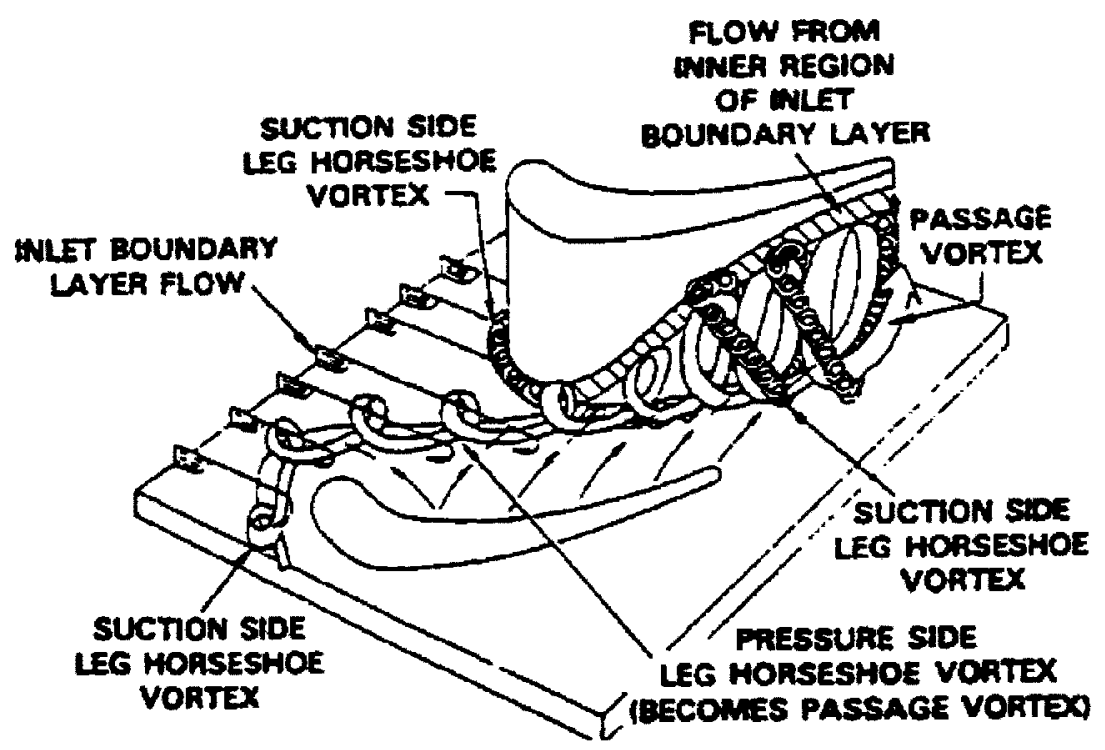

Figure 2.7: Secondary Flow Structures According to Sharma and Butler (reproduced from Sharma and Butler, 1987)

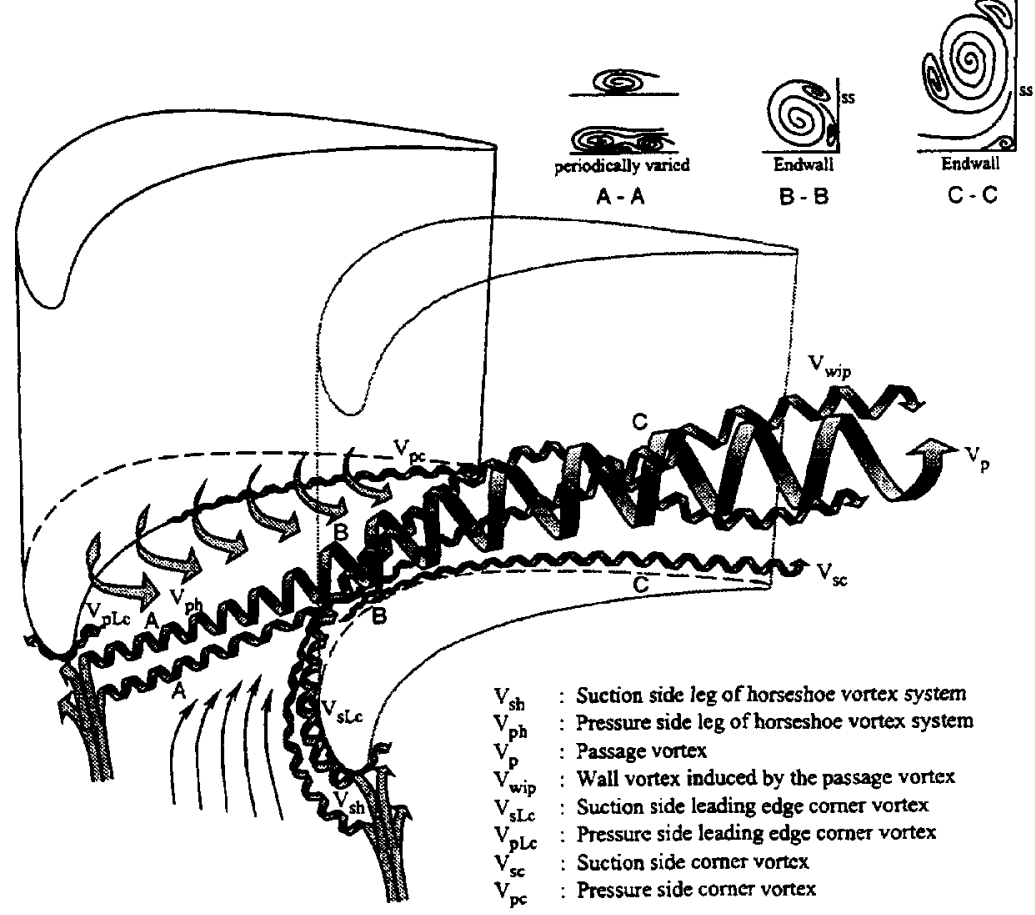

Figure 2.8: Secondary Flow Structures According to Wang et al.

(reproduced from Wang et al., 1997) 


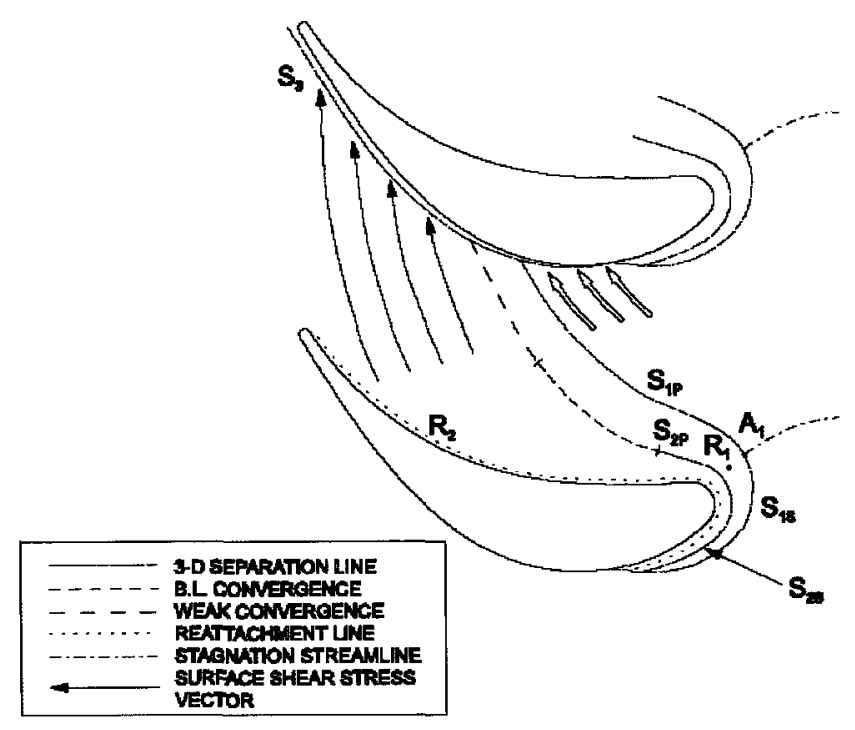

Figure 2.9: Limiting Endwall Flow Patterns

(reproduced from Benner, 2003)

the lift-off of the horseshoe vortex, respectively. The suction side separation lines, $S_{1 s}$ and $S_{2 s}$, are determined by the strong flow acceleration on the front part of the blade suction surface. At the merge point of $S_{1 s}$ and $S_{2 s}$, the suction side leg of the horseshoe vortex is driven off the endwall and up the suction surface. The $S_{3}$ separation line originates at approximately the same point. The separation lines, $S_{1 p}$ and $S_{2 p}$, are forced to migrate towards the blade suction surface by the strong pressure-to-suction surface (cross passage) pressure gradient. Sieverding (1985) believes that depending on the blade loading, the interaction between the $S_{1 p}$ and $S_{2 p}$ and the blade suction surface may be different. The effect of the loading on the secondary flows will be discussed further in Chapters 4 and 5. 


\subsection{Experimental Secondary Loss Investigations}

Endwall flows in turbomachines have been the subject of extensive experimental studies for more than half a century. Owing to the rather high share of secondary losses in total aerodynamic loss, a great number of investigators have examined the physical origins of the losses and explored the possibilities of reducing them.

A number of the previous experimental studies have already been mentioned. Table 2.1 summarizes the previous studies that the author considers relevant to the present research. More detailed discussion of some of the references will be given in connection with the results presented in Chapters 4 to 6 .

The table is organized such that for each cited reference information such as measurements details, investigated parameters and experimental conditions are given in addition to a short description of the findings. The terms and abbreviations used in the table are given below. 
Measurement location:

BP: Blade passage

D/S: Downstream

Measurement type:

Probe type:

Three-hole pressure probe (3HP)

Five-hole pressure probe (5HP)

Seven-hole pressure probe (7HP)

Kiel probe

Pitot probe

Hot film (HF)

Hot wire (HW)

Laser Doppler velocimetry (LDV)

Cross wire (XW)

\section{Loading:}

Surface static pressure taps

\section{Flow visualization:}

Oil, smoke, etc.

\section{Parameters investigated:}

Incidence (i)

Reynolds number (Re)

Mach number (Ma)

Turbulence intensity ( $\mathrm{Tu}$ )

Pitch-to-chord ratio $(\mathrm{s} / \mathrm{C})$

Aspect ratio (AR) defined as span-to-chord ratio

Inlet boundary layer (IBL) 
Flow control (FC)

\section{Experimental conditions:}

Incidence (i)

Reynolds number (Re)

Turbulence intensity ( $\mathrm{Tu}$ )

The abbreviations used in Table 2.1 are:

EW: End wall

HSV: Horseshoe vortex

LP: Low pressure

PS: Pressure surface

SKE: Secondary kinetic energy

t, tot: Total

Y: Loss coefficient
EWP: End wall profiling

LE: Leading edge

p: Profile

sec: Secondary

SS: Suction surface

TE: Trailing edge 


\begin{tabular}{|c|c|c|c|c|c|c|c|c|c|c|c|c|c|}
\hline & & & assurement & Detail & & & & arametors & & & perrmen & & \\
\hline & & TEDBR & Prabe: & Lenting & 10wive & & & & & Hide & $\frac{8 \times x \times 10^{4}}{80}$ & fur|xit & omman \\
\hline Adjour \& Dixon [1992) & & $x$ & $H W, P($ Kele $)$ & $x$ & oil, smoke & & & & & Design & 3.7 & $0.9-3.8$ & 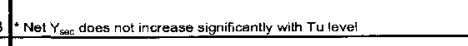 \\
\hline Aunapu et a l (2000) & $x$ & & 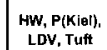 & $x$ & oil & & & & $\times$ & Design & $2.0_{1}$ & 8.0 & 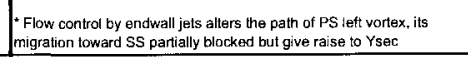 \\
\hline Becz et al (2003, 2004) & & $x$ & Prito, P(Kie) & $x$ & mylar film & & & & & Design & $5.9_{0}$ & & 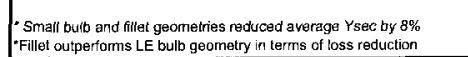 \\
\hline Benner (1997) & & $x$ & $3 \mathrm{HP}, 7 \mathrm{HP}$ & $x$ & oil & & & & & $0,10,20$ & $3.0_{1}$ & 0.4 & - New profile loss correation \\
\hline Benner (2004 a \& b ) & & $x$ & 3HP. $7 \mathrm{HP}$ & $x$ & oil & & & & & $0,10,20$ & 3.0, & 0.4 & 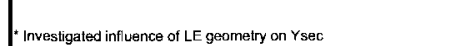 \\
\hline Benner (2005 a \& b) & & $x$ & 3HP, 7HP & $x$ & oil & & & & & $0,10,20$ & $3.0_{1}$ & 0.4 & 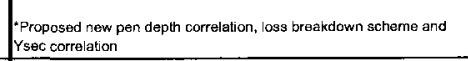 \\
\hline Brear e ta l (2002) & & $x$ & 5HP & $x$ & oil & & & & & Design & 2.10 & & 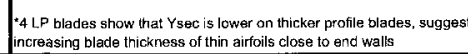 \\
\hline chen and Dixon (1986) & $x$ & $x$ & P(Kiel) & $x$ & & & & & & Design & $3.5_{0}$ & & 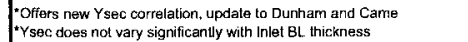 \\
\hline Cleak and Greaor-Smith (1992) & $x$ & $x$ & $\begin{array}{ll}\mathrm{HW}, \mathrm{XW}, \\
\mathrm{sH}\end{array}$ & & & & & & & Design & $40_{0}$ & 4.0 & 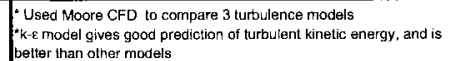 \\
\hline Je la Rosa Blanco et al (2003) & $x$ & $x$ & Pltot, 5HP & $x$ & oil & & & & & Design & 2.32 。 & & 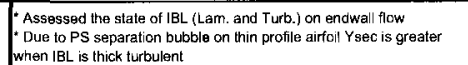 \\
\hline udden and Fotther (1997) & & $\mathrm{x}$ & $\begin{array}{c}\text { Pitot, HW, } \\
\text { SHP }\end{array}$ & $x$ & oll & & & & & Design & 1.2, 5..0 o & 1.46 .8 & 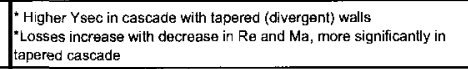 \\
\hline Juden et a (1999) & & $x$ & 5HP & $x$ & & & & & & Design & $5.0_{0}$ & & 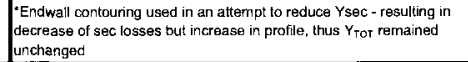 \\
\hline Dossena et al (1999) & & $x$ & Pitot, 5HP & $x$ & & & & & $x$ & Design & $10.8_{0}$ & 0.5 & 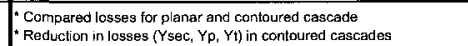 \\
\hline Oossena et al (2004) & & $x$ & Pitot, 5HP & & & & & & & 24.0 .24 & & 1.0 & 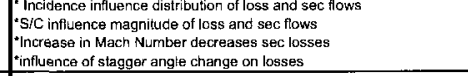 \\
\hline Gregory-Smith et a l $(1988 \mathrm{a} \&$ b) & $x$ & $x$ & HW, 5HP & & oil & & & & & Design & $5.0_{0}$ & 1.4 & 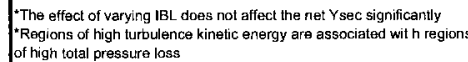 \\
\hline Sregory-Smith and Cleak (1992) & $x$ & $x$ & 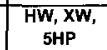 & & & & & & & Desion & $40_{0}$ & low-50 & 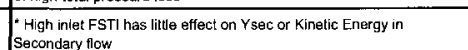 \\
\hline Sregor-Smith et al (2001) & $x$ & $x$ & $\mathrm{XHW}, 5 \mathrm{HP}$ & $x$ & oil & & & & $x$ & Design & & 5.0 & 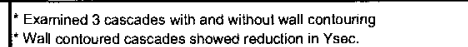 \\
\hline tarison (1990) & $x$ & $x$ & $\begin{array}{l}\text { Pitot, 3HP, } \\
\text { sHP, }, \mathrm{FF}\end{array}$ & $x$ & oil & & & & & Design & $8.4_{0}$ & 0.4 & 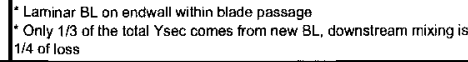 \\
\hline tavey ot al (2002) & & & 3HP & & & & & & & Design & $\begin{array}{l}\mathrm{S}: 6.3 \\
R: 1.76\end{array}$ & & 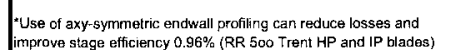 \\
\hline Hodson and Dominy (1987a) & $x$ & $x$ & Pitot, 5HP & $x$ & oil & & & & & Design & 2.90 & 0.5 & 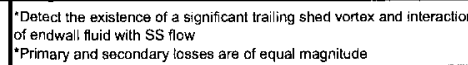 \\
\hline Hodson and Dominy (1987b) & & & Pitot, 5HP & $x$ & oil & & & & & $-20,0+9$ & $1.5-6.0_{0}$ & 0.5 & 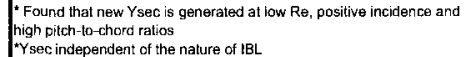 \\
\hline nd Lakshminarayana (1996) & & $\mathrm{x}$ & $\underset{5 \mathrm{sw}, \mathrm{xw}}{\mathrm{HW}}$ & & & & & & & Design & $4.0_{0}$ & 4.0 & 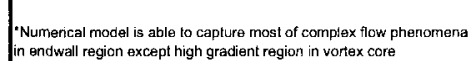 \\
\hline
\end{tabular}




\begin{tabular}{|c|c|c|c|c|c|c|c|c|c|c|c|c|c|c|c|}
\hline & & Met & suremen & Dotails & & & & Paran & neters & & & & perinon & & \\
\hline Roforonce: & $\frac{1}{3}$ & TEDS: & EDrobe & Fosoung & Fow dia & & & ative & Wed & AlEil|: & & idegle & Nondition & $\frac{5}{\sin (x)}$ & Comments \\
\hline Ingram et al (2002) & $x$ & $\mathbf{x}$ & $5 \mathrm{HP}$ & & oil & & & & & & $x$ & Design & & & $\begin{array}{l}\text {-Experimented with planarar and axy-symmetric encowall treatment } \\
\text { cascades, EWP can reduce Ysecc of up to } 24 \%\end{array}$ \\
\hline Ingram et al (2005) & $\mathbf{x}$ & $\mathbf{x}$ & $3 \mathrm{HP}, 5 \mathrm{HP}$ & & oil & & & & & & $x$ & Design & $4.0_{0}$ & 5.0 & 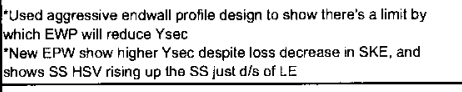 \\
\hline Kawai (1994) & & $\mathbf{x}$ & XW, $\mathbf{P ( K i e l )}$ & & oil & & & & & & $x$ & Design & $4.5_{\circ}$ & & 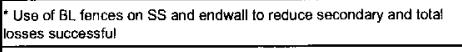 \\
\hline Langston et al (1977) & $\mathbf{x}$ & $\mathbf{x}$ & HW, 5HP & $\mathbf{x}$ & oil & & & & & & & Design & $10.0_{\circ}$ & 1.0 & 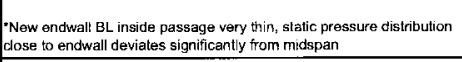 \\
\hline Langston (1980) & $\mathbf{x}$ & $\mathbf{x}$ & HW, 5HP & $\mathbf{x}$ & oil & & & & & & & Design & $10.0_{0}$ & 1.0 & 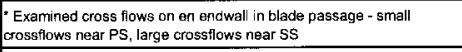 \\
\hline Langston (2001) & & & & & & & & & & & & & & & $\begin{array}{l}\text { A review of secondary flows up to } 2001 \text { and melhods tor reducing } \\
\text { Ysoc }\end{array}$ \\
\hline Moore and Achye (1985) & $\mathbf{x}$ & $x$ & $3 \mathrm{HP}, 5 \mathrm{HP}$ & & & & & & & & & Design & $5.2_{0}$ & 0.2 & 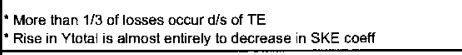 \\
\hline Moore and Moore (1985) & $x$ & $\mathbf{x}$ & & & & & & & & & & Design & $5.2,10.0 \mathrm{o}$ & & 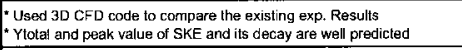 \\
\hline Moustapha et al (1985) & & $\mathbf{x}$ & Pitot, 5HP & $x$ & oil & & & & & & & Design & $3.0-6.0$ & 4.0 & $\begin{array}{l}\text { Measured losses in linear and annular cascade, measuring higher } \\
\text { osses and underturring in anrular cascade }\end{array}$ \\
\hline Moustapha et al (1990) & & & & & & & & & & & & & & & $\begin{array}{l}-\begin{array}{l}\text { - Improved loss prediction at off-design incidence for profile and } \\
\text { secondary losses }\end{array} \\
\end{array}$ \\
\hline Perdichizzi (1990) & & $x$ & Pitot, 5HP & $\mathbf{x}$ & & & & $\mathbf{x}$ & & & & Design & & & $\begin{array}{l}\text { - As Mach number increases, the passage vortex migrates towards } \\
\text { endwall, Ysec is a smaler fraction of Yrotala }\end{array}$ \\
\hline Perdichizzi and Dossena (1993) & & $\mathbf{x}$ & & $x$ & oil & $x$ & & & $x$ & & & -60 to +35 & $8.4_{0}$ & 0.9 & 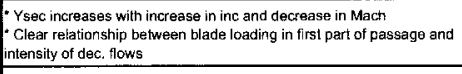 \\
\hline Sauer at al (2001) & & $x$ & Pitot, 5HP & & & & & & & & $x$ & Design & $4.5_{0}$ & $<1.0$ & 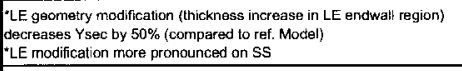 \\
\hline Sharma and Butler (1987) & & & & & & & & & & & & & & & $\begin{array}{l}\text { - Secondary fow correlation } \\
\text { - Penetration deplh }\end{array}$ \\
\hline Sthith and Lin (2003) & $\mathbf{x}$ & $\mathbf{x}$ & & & & & & & & & $x$ & $-30,0,30$ & 43.8, & 10.0 & 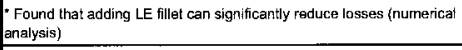 \\
\hline Sievercing and Van den Bosche (1983) & & & & & smoke & & & & & & & Design & & & - Flow viz of secondar How structure \\
\hline Sieverding et al (1984) & $x$ & $\mathbf{x}$ & $4 \mathrm{HP}$ & & smoke & & & & & & & Design & $0.98_{1}$ & 0.8 & \begin{tabular}{|l} 
"Investigaled velocity profiles and total pressure distributions within \\
and diss of blade passage in annular cascade.
\end{tabular} \\
\hline Sieverding (1985) & & & & & & & & & & & & & & & -Summary of fiterature on secondary flows \\
\hline Walsh and Gregory-Smith (1990) & $x$ & $x$ & $3 \mathrm{HP}, 5 \mathrm{HP}$ & $\mathbf{x}$ & oil & & & & & $\mathbf{x}$ & & Design & $4.0_{0}$ & & 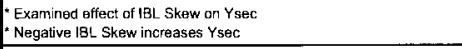 \\
\hline Wang at al (1997) & & & & & smoke & & & & & & & Design & $0.27_{\circ}$ & 0.2 & 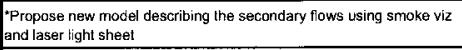 \\
\hline Weiss and rottner (1995) & $\mathbf{x}$ & $\mathbf{x}$ & Pitot, 5HP & $x$ & oil & & & & & & & Design & $5.0_{0}$ & 4.0 & 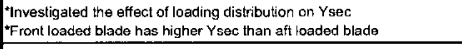 \\
\hline Yammamoto (1987) & $x$ & $x$ & Pitot, 5HP & & & & & & & & & Design & $1.8_{0}, 2.8_{0}$ & 0.5 & 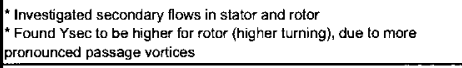 \\
\hline Yamamoto and Nouse (1988) & $x$ & $x$ & Pitot, 5HP & $x$ & oil, smoke & & & & & & & $\begin{array}{c}-53.3 \text { to } \\
7.2 \\
\end{array}$ & $1.85_{\mathrm{o}}$ & & $\begin{array}{l}\text { Ysec increase wilh increasing incidence due to stronger passage } \\
\text { vortex } \\
\text {-Athegative incidence losses mailly due to separation ar PS }\end{array}$ \\
\hline
\end{tabular}




\subsection{Concluding Remarks}

Researchers have been investigating secondary flows in turbomachines for more than half a century. Many of those studies have been focused on gaining a better understanding of the flow physics and giving a more precise picture of secondary flow behavior (Hawthorne (1955), Sieverding (1985), Sharma and Butler (1987), Langston et al. (1977), and Wang et al. (1997)). Other investigators (Sharma and Butler (1987), Benner (2003), Dunham and Came (1970), and Kacker and Okapuu (1982)), have concentrated their efforts on predicting the secondary losses, and their work has resulted in empirical or semi-empirical correlations that may be used in the early design stages for loss estimation. More recently much research effort, i.e. Aunapuu et al. (2000), Gregory-Smith et al. (2001), Becz et al. (2003) and (2004), Duden and Fottner (1999), Ingram et al. (2005), Sauer et al. (2001), has been devoted to finding ways to mitigate the secondary losses by employing various flow control techniques. However, not all questions pertaining to secondary flows have been answered, and the picture continues to evolve.

With the industry's demand for more economical design, turbine designers are faced with the challenge of designing turbomachines that are able to deliver the same power and performance with a reduced number of blades. Bearing in mind that the losses due to endwall flows may account for up to $1 / 3$ of the total aerodynamic losses for conventional blade designs, it is expected that more effort will be invested into characterization of impact of reduced number of blades on secondary losses. 


\section{Chapter 3}

\section{Experimental Set-up and Measurement Methods}

\subsection{Introduction}

This chapter describes the experimental set-up, equipment and measurement procedures. It also discusses data reduction methods and uncertainty analysis involved with this experiment.

The present experimental investigation was conducted in an open-circuit wind tunnel located in Low-Speed Turbomachinery Laboratory (LSTL) at Carleton University. The chapter will start with a description of the wind tunnel followed by details of the test section, turbulence generating grids and test cascades. Instrumentation used in this investigation is described in Section 3.5, while a description of the pressure probes is given in Section 3.6. The following section outlines the cascade testing methods. Finally, the chapter ends with a discussion of the data reduction procedures. 


\subsection{Low-Speed Wind Tunnel}

Figure 3.1 shows a schematic layout of the low-speed, open-circuit wind tunnel at Carleton University. The tunnel was commissioned by Rodger (1992). The air is supplied using a 40HP radial blower driven by a 4-pole AC motor. The maximum air flow rate is approximately $5 \mathrm{~kg} / \mathrm{s}$ with the corresponding total pressure rise of about $1.2 \mathrm{kPa}$. The swirl present at the exit of the blower is removed by a honeycomb.
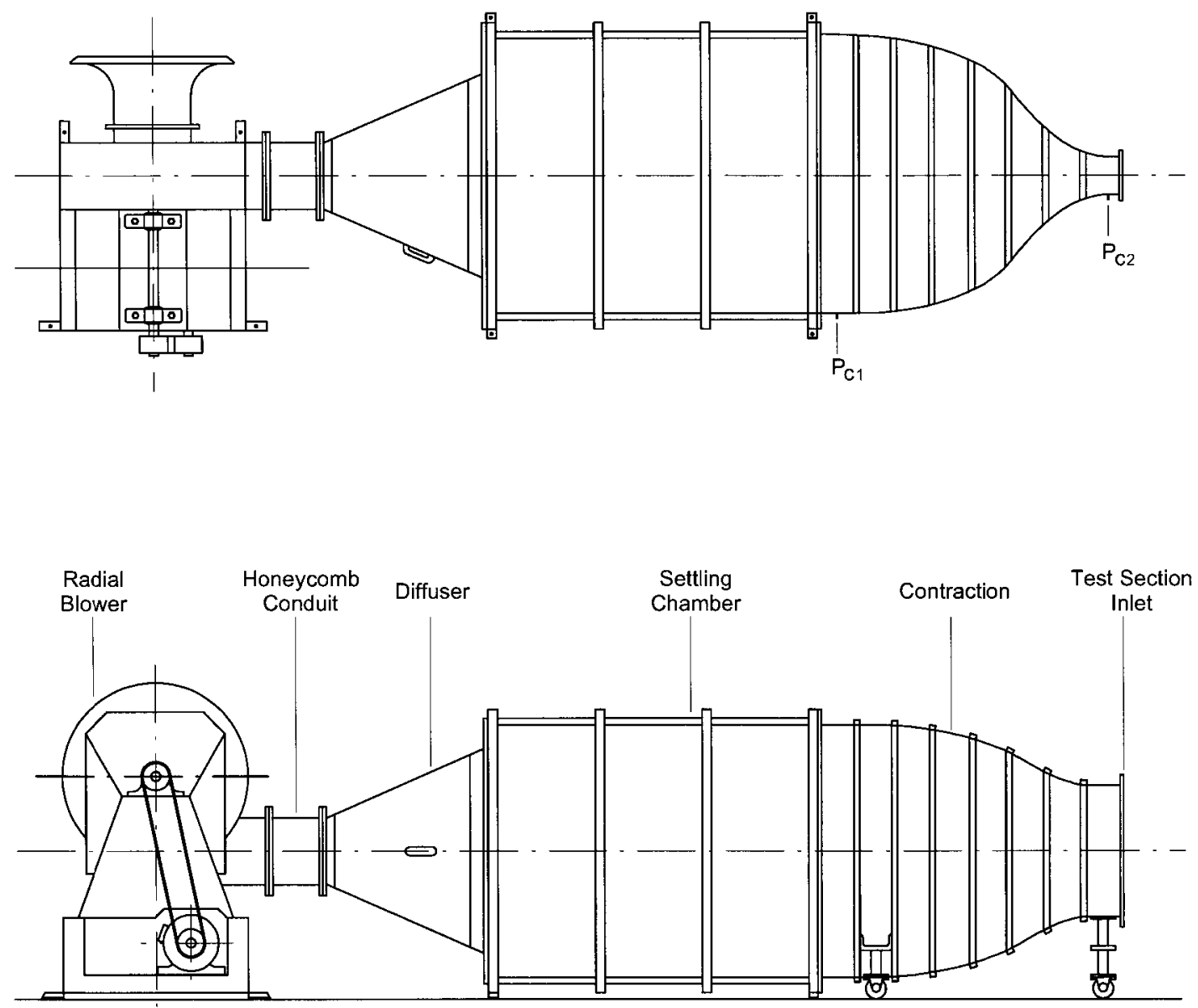

Figure 3.1: Carleton University Low-Speed Wind Tunnel (reproduced from Mahallati, 2003) 
Before being discharged into the settling chamber, the flow is diffused through a square diffuser with an $19^{\circ}$ divergence angle and an area ratio of 5.9. Five evenly spaced screens in the diffuser and four screens in the settling chamber ensure the uniformity of the flow at the test section inlet as well as reduction in the turbulence scale. A square to rectangular convergent section with a contraction ratio of 14:1 accelerates the flow into the test section. The previous researchers quoted maximum attainable velocity at the inlet of the test section of about $30 \mathrm{~m} / \mathrm{s}$.

The wind tunnel operating point is set using the static pressure taps $P_{C 1}$ and $P_{C 2}$ located at the inlet and outlet of the contraction section, respectively. These two taps also provide the reference pressures for the differential pressure transducers. These topics will be further discussed in Sections 3.5.1 and 3.7.1.

\subsection{Test Section}

The test section shown in Figure 3.2 was used for the present measurements. It was designed and commissioned by Goobie (1989). It is a variable incidence linear cascade connected to the wind tunnel described previously. It can be used for both design- and off-design incidence measurements $\left( \pm 30^{\circ}\right)$ by rotating the turntable. The inlet to the test section is of rectangular cross-section with dimensions of $67.3 \times 20.0$ $\mathrm{cm}$. The turntable is mounted on the back endwall, while a removable plexiglass window, not shown in Figure 3.2, forms the front endwall.

Seven control surfaces and a blockage mechanism are used to set the uniformity and the periodicity of the inlet and outlet flow, respectively. The blockage mechanism was designed by Mahallati (2003) in order to improve the inlet flow uniformity in the top part of the cascade. The procedure for setting the inflow uniformity and outflow 

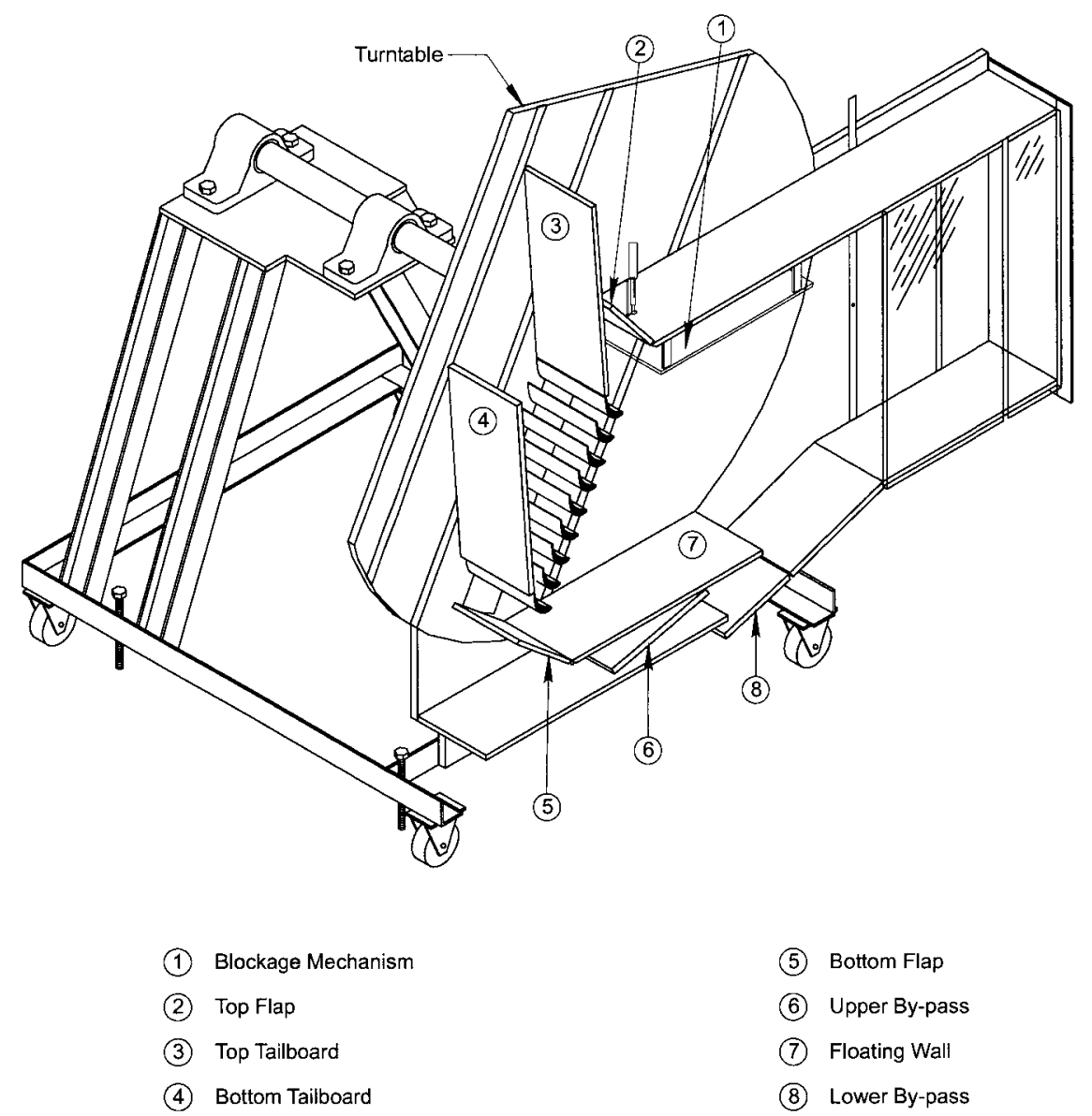

(5) Bottom Flap
(6) Upper By-pass
(7) Floating Wall
(8) Lower By-pass

Figure 3.2: Carleton University Low-Speed Wind Tunnel Test Section (reproduced from Mahallati, 2003)

periodicity will be described in Section 3.7.2.

The probes used in the measurements are moved by means of a traverse mechanism mounted on the back side of the turntable. The mechanism provides two degrees of freedom movement for the pressure probes with a minimum step size of $0.00635 \mathrm{~mm}$ in both directions.

The background freestream turbulence intensity (FSTI) of about $0.4 \%$ was reported by a couple of previous investigators (Mahallati (2003), Benner (2003)) for 
this wind tunnel. The FSTI levels more representative of the real engine environment (3-7\%, private communications with PWA, East Hartford, CT, 2004) were achieved by means of turbulence generating grids installed upstream of the cascade.

Two turbulence generating grids, giving FSTI values of $1.5 \%$ and $4.0 \%$, were employed in the present measurements. The mounting positions for the generating grids are shown in Figure 3.3. The $1.5 \%$ and $4.0 \%$ grids are mounted in positions 1 and 2 seen in Figure 3.3, respectively. Due to the proximity of the grids and the fact that the flow has not fully mixed-out prior to reaching the test cascades, particularly for position 2, flow non-uniformities were observed at the inlet traverse plane (position 3). The issue of inlet flow non-uniformity due to the grids will be further addressed in Chapters 4 and 5.

\subsection{Test Cascades}

Three cascades designed by Pratt \& Whitney Aircraft (PWA) were used in the present measurements. The cascades denoted as PAKB, PAKD-A and PAKD-F belong to the same family of highly loaded low-pressure turbine (LPT) airfoils and are designed for the same duty. All airfoils share the same design inlet and outlet flow angles of $35^{\circ}$ and $60^{\circ}$, respectively. The test cascades can be seen in Figure 3.4, while the relevant geometrical data are summarized in Table 3.1.

The PAKB airfoil is representative of a midspan section of an LPT vane employed by a current PWA engine, and is the baseline of the family. It was inversely-redesigned for low-speed testing. The PAKB cascade consists of nine blades with pitch-to-chord ratio of 0.796 and the Zweifel number of 1.08. The PAKD airfoils are the derivatives of the baseline blade. They are $25 \%$ more highly loaded than the baseline blade, with 


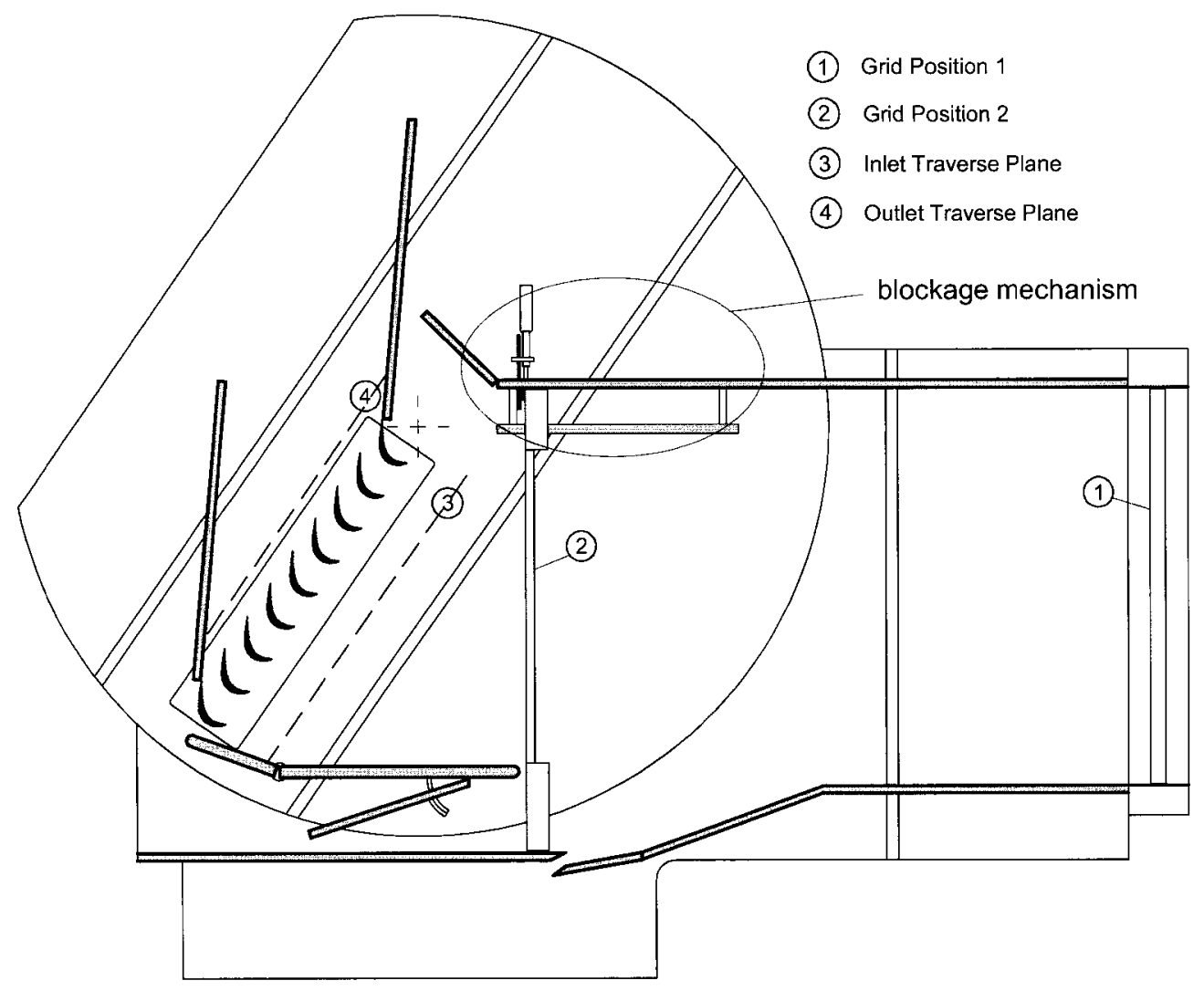

Figure 3.3: Grid Mounting Positions and Traverse Planes

(reproduced from McAuliffe, 2003)

a Zweifel number of 1.36 and the pitch-to-chord ratio of 0.993 and 0.901 for PAKD-A and PAKD-F, respectively. The two more highly loaded airfoils are designed such that the PAKD-A airfoil is aft-loaded, and PAKD-F is front-loaded. Both PAKD cascades contain seven blades. The difference in loading distribution is achieved mainly by changes in the stagger angle (the greater the stagger angle, the more forward-loaded is the airfoil) and the blade thickness distribution. 


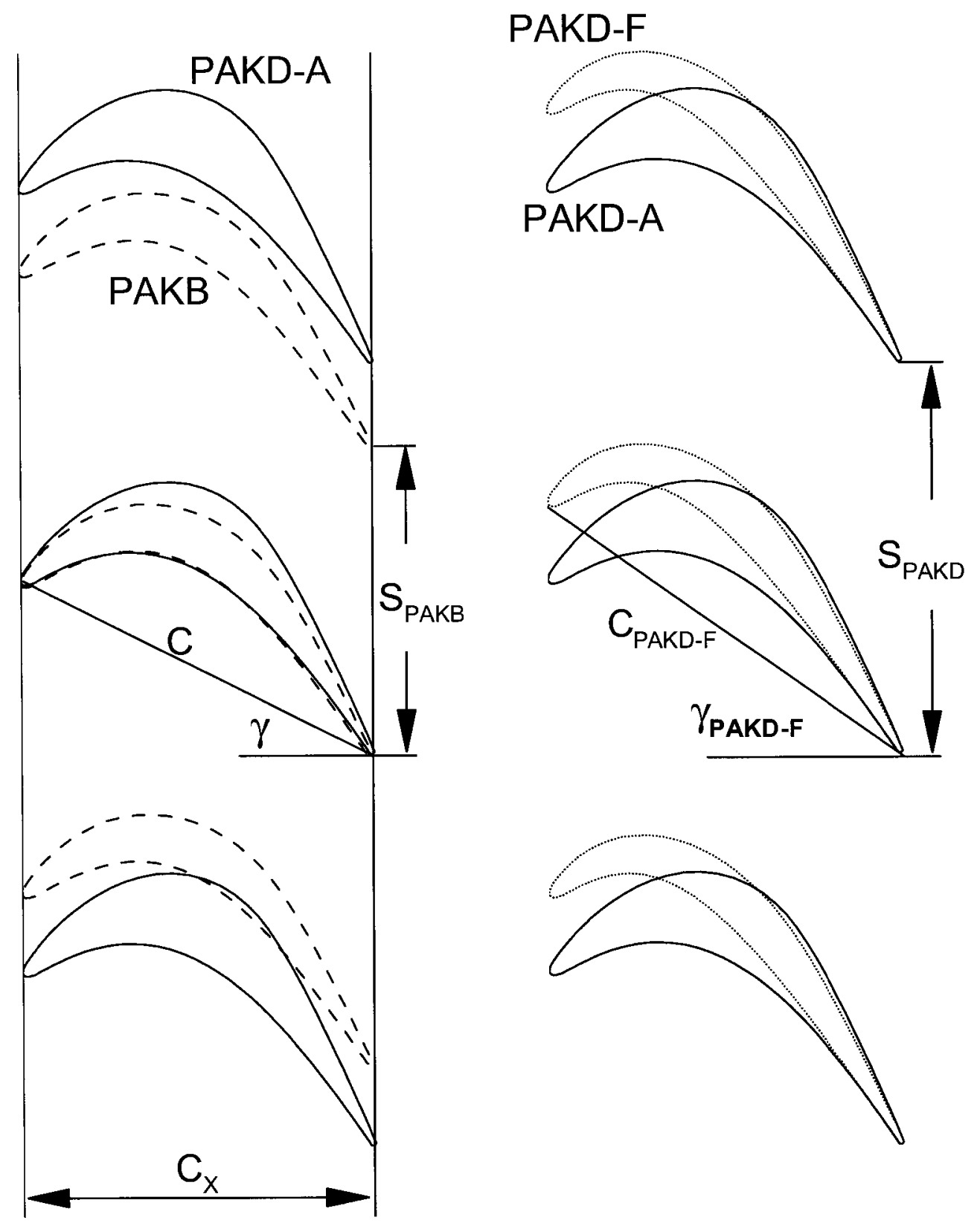

Figure 3.4: Airfoil Comparison 
Table 3.1: Summary of Cascade Data

\begin{tabular}{|l|c|c|c|}
\cline { 2 - 4 } \multicolumn{1}{c|}{} & PAKB & PAKD-A & PAKD-F \\
\hline Blade Span $(\mathbf{h})[\mathbf{m m}]$ & 203.2 & 203.2 & 203.2 \\
\hline Blade Pitch $(\mathbf{s})[\mathbf{m m}]$ & 66.8 & 84.2 & 84.2 \\
\hline Aspect Ratio $(\mathbf{h} / \mathbf{c})$ & 2.42 & 2.40 & 2.17 \\
\hline Axial Chord Length $\left(\mathbf{C}_{\mathbf{x}}\right)[\mathbf{m m}]$ & 75.4 & 76.2 & 76.2 \\
\hline True Chord Length $(\mathbf{C})[\mathbf{m m}]$ & 83.9 & 84.8 & 93.5 \\
\hline Stagger Angle $(\boldsymbol{\gamma})$ & $26.0^{\circ}$ & $26.0^{\circ}$ & $35.4^{\circ}$ \\
\hline Design Inlet Flow Angle $\left(\boldsymbol{\alpha}_{\mathbf{l d e s}}\right)$ & $35.0^{\circ}$ & $35.0^{\circ}$ & $35.0^{\circ}$ \\
\hline Design Outlet Flow Angle $\left(\boldsymbol{\alpha}_{\mathbf{2 d e s}}\right)$ & $60.0^{\circ}$ & $60.0^{\circ}$ & $60.0^{\circ}$ \\
\hline Maximum Thickness $\left(\mathbf{t}_{\mathbf{m a x}}\right)[\mathbf{m m}]$ & 10.7 & 16.4 & 9.7 \\
\hline Trailing Edge Thickness $\left(\mathbf{t}_{\mathbf{T E}}\right)[\mathrm{mm}]$ & 1.1 & 1.1 & 1.1 \\
\hline Solidity $(\boldsymbol{\sigma})$ & 1.26 & 1.01 & 1.11 \\
\hline Zweifel coefficient $\left(\mathbf{Z}_{\mathrm{w}}\right)$ & 1.08 & 1.36 & 1.36 \\
\hline Number of Airfoils in $\mathbf{C a s c a d e}$ & 9 & 7 & 7 \\
\hline
\end{tabular}

\subsection{Experimental Instrumentation}

\subsubsection{Pressure Transducers}

The pressure measurements for the present experiments were performed with differential transducers. Three types of transducers were used with operating ranges of $\pm 250 \mathrm{~Pa}, \pm 1250 \mathrm{~Pa}$ and $\pm 5000 \mathrm{~Pa}$. The types and models are given in Table 3.2 .

Table 3.2: Pressure Transducers

\begin{tabular}{|c|c|c|}
\hline Pressure Range (Pa) & Manufacturer & Transducer \\
\hline \pm 250 & Data Instrument & DRAL501DN \\
\hline \pm 1250 & Data Instrument & DRAL505DN \\
\hline \pm 5000 & Sensor Technique & 113LU20D-PCB \\
\hline
\end{tabular}


The pressure transducers exhibit a linear relationship between the measured pressure difference, $\Delta P$, and the output voltage, $\mathrm{E}$, as given by:

$$
\Delta P=C_{1} E+C_{2}
$$

where $C_{1}$ and $C_{2}$ are the calibration coefficients obtained from the calibration curves. The calibration data for the pressure transducers are given in Appendix A.

\subsubsection{Temperature Monitor}

An Omega i-series temperature/process meter, model DPi8-C24, connected to a type- $T$ (Copper-Constantan) thermocouple was used to read the temperature in the test section. Mahallati (2003) reported $\pm 0.4^{\circ}$ accuracy in the temperature readings. The recorded temperatures were used in determining the air density and viscosity.

\subsubsection{Digital Barometer}

A digital barometer, Omega model DPI740, which incorporates a pre-calibrated 77 to $115 \mathrm{kPa}$ absolute pressure transducer was used to measure the ambient pressure. The transducer has an accuracy of $\pm 0.02 \%$. The ambient pressure was used in addition to the test section flow temperature and wind tunnel contraction pressure difference to set the wind tunnel operating point (Reynolds number) for each measurement, as will be discussed in Section 3.7.1. 


\subsubsection{Data Acquisition System}

The data acquisition was performed using a computerized system which consists of a sample-and-hold PCI card, PowerDaq II, installed into a personal computer and an appropriate in-house software package, named CarletonDaq.

The PowerDaq II was made by the United Electronics Industries (UEI), the model number PD2-MFS-8-800/14 (14-bit resolution). The card was used to acquire signals from up to eight pressure transducers simultaneously, and stream the data to the hard drive.

The CarletonDaq is a Windows based software package written in Visual Basic. It was used to perform the data acquisition and to control traverse gear of movements. The software has a built-in Reynolds number calculator that was used to set the operating point of the wind tunnel. In addition, the data reduction for the three-hole probe can also be performed by the CarletonDaq.

More details are available from Mahallati (2003), who developed and extensively tested the system.

\subsubsection{Probe Calibration Rig}

An in-house calibration rig presented in Figure 3.5 was used for the calibration of the pressure probes used for the present experiment. The air is drawn through a nozzle by a suction type radial blower equipped with a throttle valve. The maximum achievable velocity at nozzle outlet is approximately $45 \mathrm{~m} / \mathrm{s}$. The probe is mounted on the two-axis motorized turntable that allows pitch and yaw movement of approximately $\pm 50^{\circ}$. Reference total and static pressures are measured at the 
calibration rig wall and nozzle exit, respectively. Benner (2003) and Mahallati (2003) have provided more information on the flow quality and the calibration rig itself.

The rig was used for the calibration of three- and seven-hole probes. The pressure probes will be described in the next section, while the calibration data for the probes will be provided in Appendices B and C, respectively.

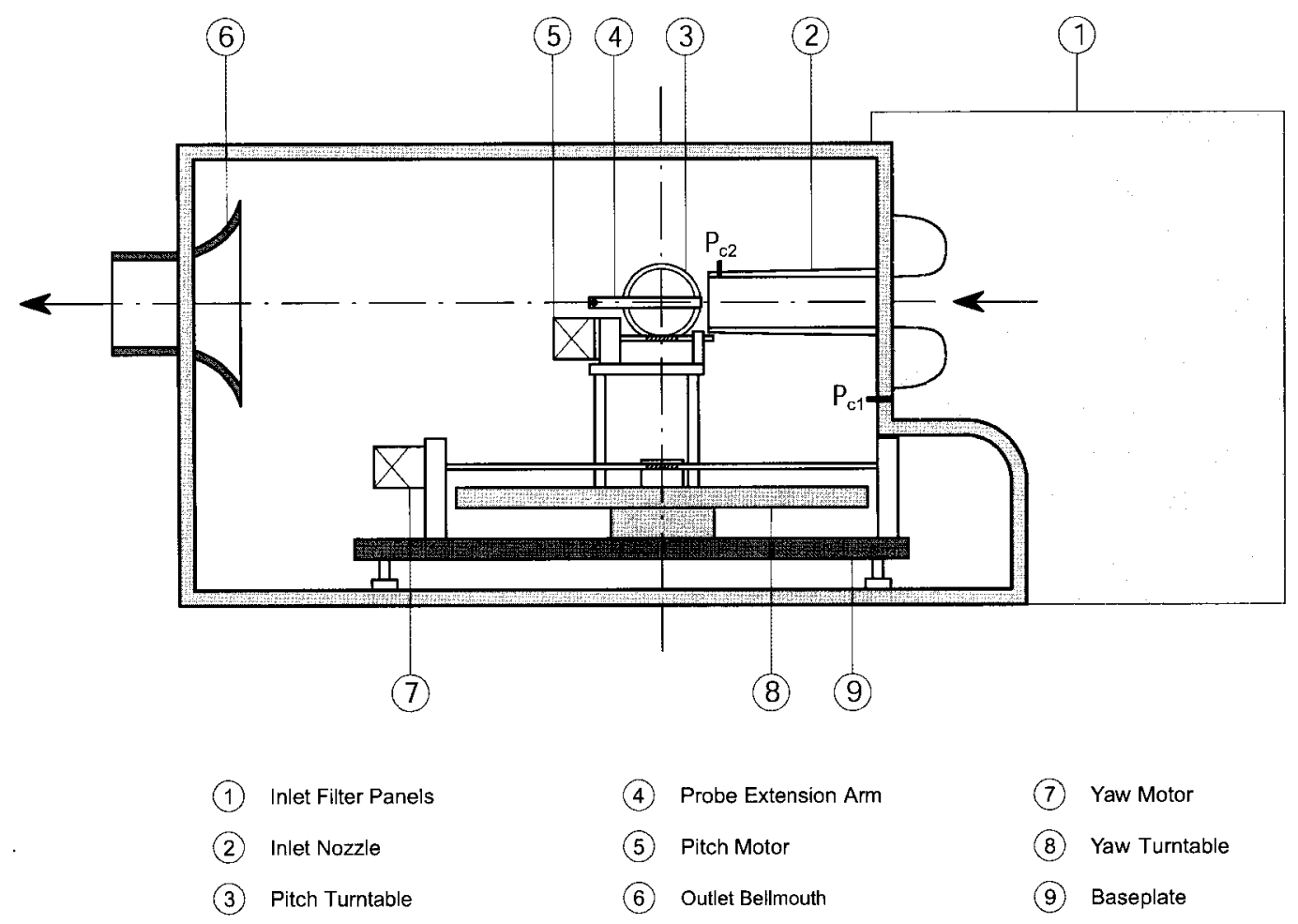

Figure 3.5: Calibration Rig

(reproduced from Mahallati, 2003) 


\subsection{Pressure Probes}

\subsubsection{Pitot Tube}

A Pitot tube was used to traverse the inlet boundary layer. All measurements were performed at a location $1.2 C_{x}$ upstream of the blade leading edges. The pitot tube has an inner diameter of $0.33 \mathrm{~mm}$ and outer diameter of $0.68 \mathrm{~mm}$. The diameter is approximately $3.5 \%$ and $2.0 \%$ of the boundary layer thickness, for FSTI of $1.5 \%$ and $4.0 \%$, respectively. The probe has a hollow cylindrical stem which can be fixed to the traverse mechanism located on the back side of the endwall. The Pitot tube is made of stainless steel and is bent that the tip of the probe contacts the endwall first. The Pitot tubes used in the LSTL were thoroughly tested by Mahallati (2003) and Benner (2003) who concluded that the uncertainty in the measured total pressure is about $0.5 \%$ of the reference dynamic pressure.

\subsubsection{Three-Hole Probe}

A three-hole probe was used throughout the measurements to measure the twodimensional flow (at the midspan) up and downstream of the cascade. The probe used for the measurements was manufactured by Mahallati (2003) and is shown in Figure 3.6. The probe has three stainless steel hypodermic tubes with a $45^{\circ}$ wedge tip. The probe tip width is approximately $3 \%$ and $2.4 \%$ of the PAKB ad PAKD blade pitches respectively. The dynamic pressure, static pressure and flow angle can be inferred from the three measured pressures. The reduction of data obtained from three-hole measurements was performed by the CarletonDaq software. The calibration curves are given in Appendix B. 

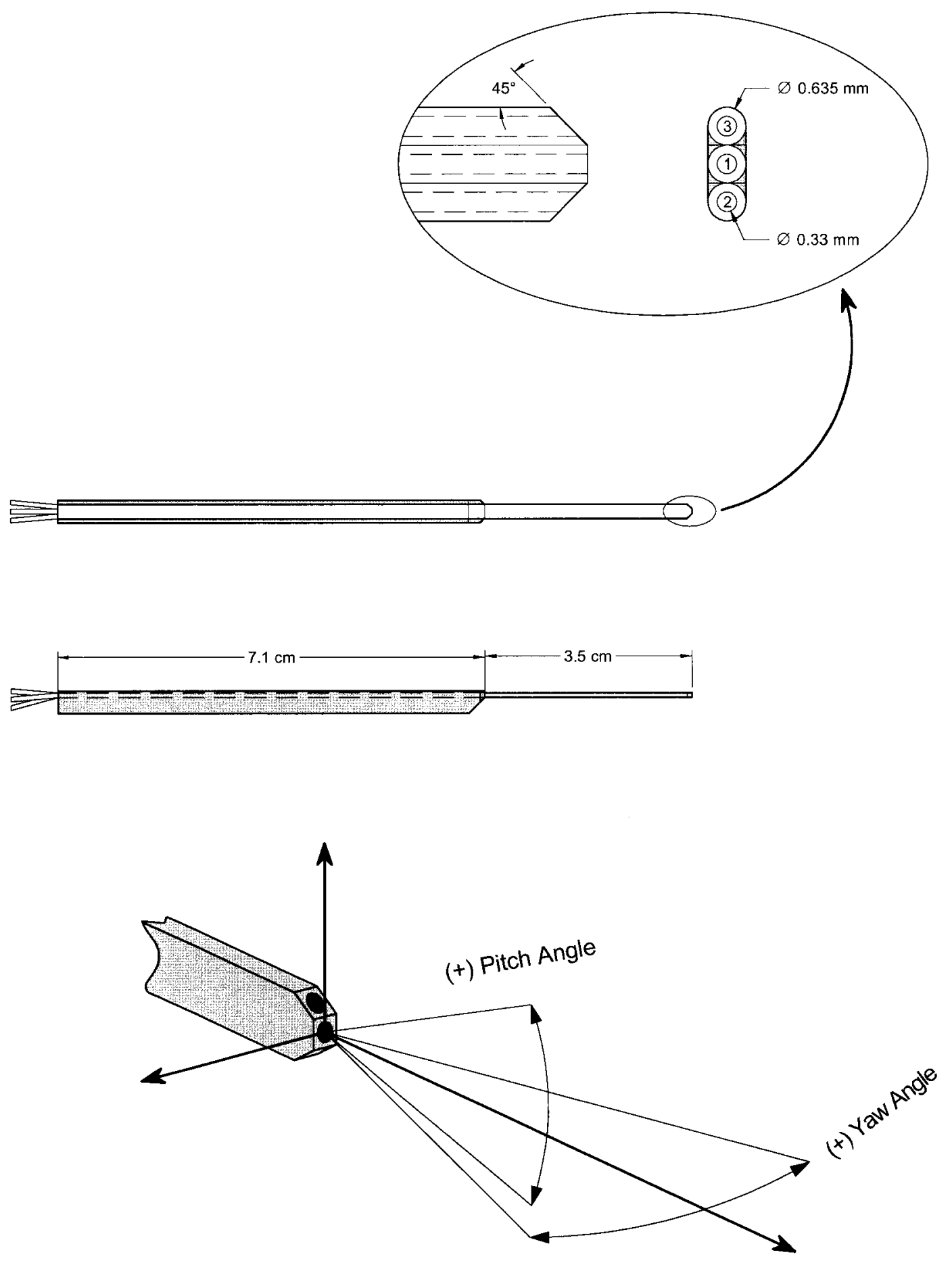

Figure 3.6: Three-Hole Probe

(reproduced from Mahallati, 2003) 


\subsubsection{Seven-Hole Probe}

Three-dimensional flows such as those measured in this experiment cannot be measured with three-hole probes. To be able to extract all three velocity components and corresponding flow angles, one must use multi-hole probes (i.e. five- or sevenhole probes). A seven-hole probe with a $2.4 \mathrm{~mm}$ tip diameter was used to measure the flow field up and downstream of the blade passage. Figure 3.7 gives a schematic view of the probe. The probe consists of seven stainless steel tubes. The six tubes are placed $60^{\circ}$ apart circumferentially around the middle one. The figure also shows the convention used for pressure hole numbering used in the present measurements.
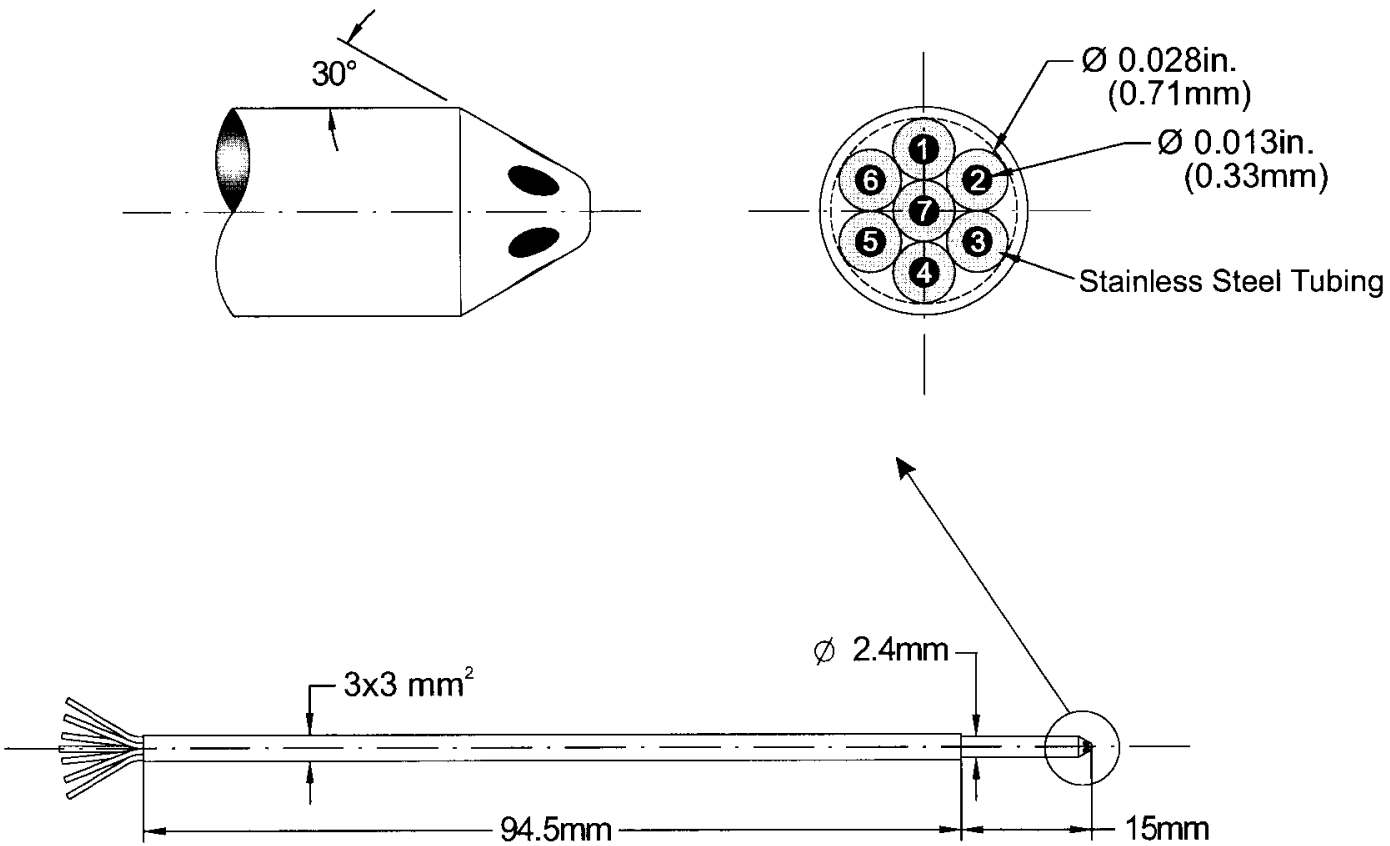

Figure 3.7: Seven-Hole Probe

(reproduced from Benner, 2003) 
The probe was calibrated over a range of $\pm 50^{\circ}$ in a known flow in the calibration rig discussed in Section 3.5.5. The calibration procedure will be discussed in more detail in Appendix C.

\subsection{Cascade Testing Procedures}

\subsubsection{Wind Tunnel Operating Conditions}

The present measurements were performed at a constant Reynolds number of 80,000 based on the inlet velocity and axial chord as defined in equation 3.1.

$$
R e=\frac{\rho V_{1} C_{x}}{\mu}
$$

where density, $\rho$, and viscosity, $\mu$, were calculated in one of the CarletonDAQ's subroutines. The subroutine uses the test section temperature and atmospheric pressure, which were recorded by a thermocouple and digital barometer (described in Sections 3.5.2 and 3.5.3, respectively). The inlet velocity can be obtained through the following expression:

$$
V_{1}=\sqrt{\frac{2 C_{Q W T}\left(P_{C 1}-P_{C 2}\right)}{\rho}}
$$

where $P_{C 1}-P_{C 2}$ is the contraction pressure difference (Section 3.2). The pressure difference is a direct function of the fan's rotational speed. Depending on the ambient conditions, the Re had to be adjusted by varying the speed of the fan prior to each set of measurements. The wind tunnel calibration coefficient, $C_{Q_{W T}}$, is defined in equation 3.3. It was obtained experimentally for each case, since its value is different 
for each cascade, turbulence generating grid and incidence. In order to calculate the coefficient, the dynamic pressure at the inlet midspan, $q_{C L}$, was measured for each case by three-hole probe traverses at the inlet midspan.

$$
C_{Q_{W T}}=\frac{q_{C L}^{\prime}}{P_{C 1}-P_{C 2}}
$$

\subsubsection{Inlet Flow Uniformity and Outlet Flow Periodicity}

A linear cascade consists of a number of identical blades that are parallel to each other and evenly spaced. In order to obtain a nearly periodic flow, such as the one that exists in the real engine or an annular cascade, an infinite number of blades is required. In reality, even with a limited number of blades a periodic flow can be ensured for the middle blades through the use of control surfaces.

The inlet flow uniformity and outlet flow periodicity were measured using the three-hole probe described in Section 3.6.2. The probe was traversed at cascade midspan over four and six blade pitches for the PAKD and PAKB blades, respectively.

For the inlet flow uniformity the three-hole probe was traversed at a plane located $1.2 C_{x}$ upstream of the blade leading edge, and for the outlet flow periodicity the probe tip was located $0.4 C_{x}$ downstream of the blade trailing edge. Typical inlet flow uniformity and outlet flow periodicity distributions are presented in Figures 3.8 and 3.9. The figures show the flow angles, dynamic, static and total pressure coefficients for both the inlet and outlet traverses.

The inlet flow uniformity was set adjusting the flaps and the blockage system previously shown in Figure 3.2 . 

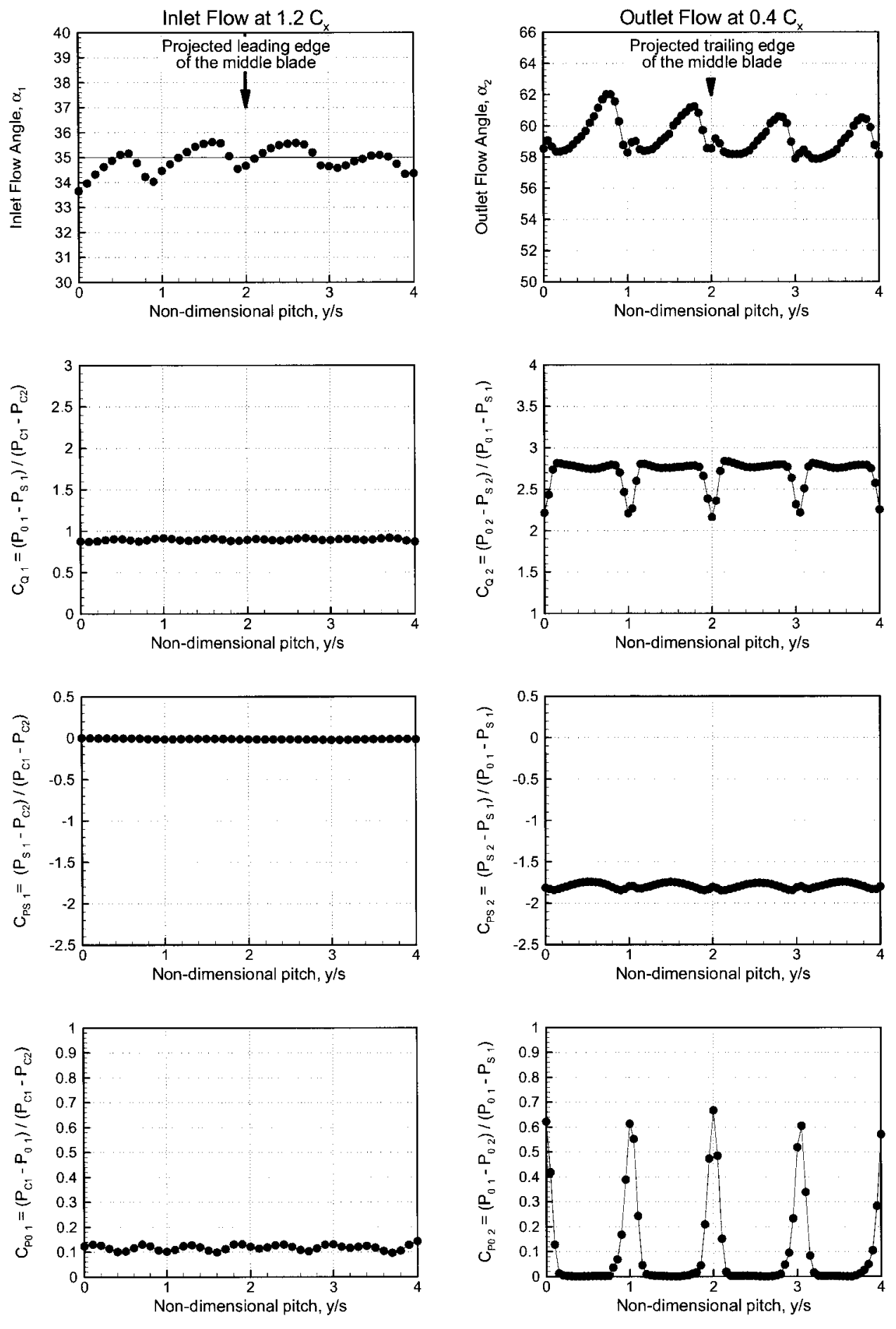

Cascade Top

Cascade Bottom

Cascade Top

Cascade Bottom

Figure 3.8: Inlet Flow Uniformity and Outlet Flow Periodicity

$\left(\mathrm{PAKD}-\mathrm{F}: \mathrm{FSTI}=1.5 \%\right.$ and Incidence $\left.=-5^{\circ}\right)$ 

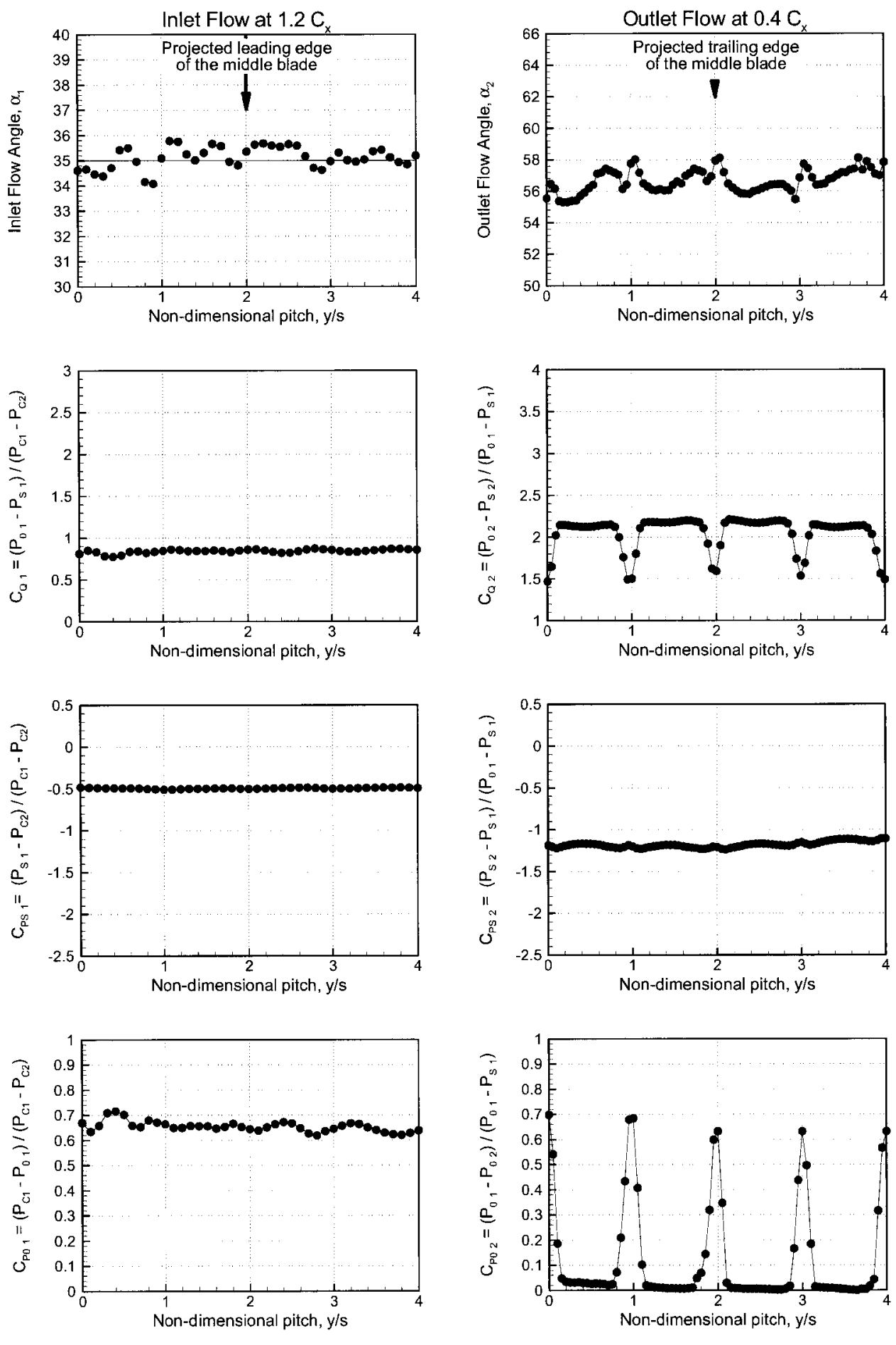

Cascade Top

Cascade Bottom

Cascade Top

Cascade Bottom

Figure 3.9: Inlet Flow Uniformity and Outlet Flow Periodicity

$\left(\right.$ PAKD-F: FSTI $=4 \%$ and Incidence $\left.=+5^{\circ}\right)$ 
Tailboards were adjusted to achieve the outlet flow periodicity. A number of iterations was required, especially for the stalled cases where a strong coupling was observed between the control surfaces. The iteration process was sped up by using the control surface and blockage mechanism settings obtained in previous measurements.

The spanwise uniformity and the non-uniformities arising from the usage of turbulence generating grids will be further discussed in Chapters 4 and 5 .

\subsubsection{Seven-Hole Probe Area Traverses}

Seven-hole probe area traverses of the flow field were performed at locations $1.2 C_{x}$ upstream of the blade leading edge and $0.4 C_{x}$ downstream of the blade trailing edge, respectively. The measurement matrix covered half span and 1.2 blade pitches (an additional $10 \%$ of the pitch on either side of the blade passage was traversed) for each measured case. Schematic view of the measurement planes and the matrix are shown in Figures 3.10 and 3.11, respectively.

The measurement interval of approximately $3.0 \%$ and $2.4 \%$ of the blade pitches for PAKB and PAKD blades respectively resulted in a 952 measurement points for the PAKB cascade and 1190 points for the PAKD cascades. For each point 15,000 samples were taken at a sampling rate of $1 \mathrm{kHz}$. This was determined based on examining the cumulative average of the pressure signals. The waiting time between each measurement point is about eight seconds after the probe has been moved to a new location by the traverse mechanism. On the average each run lasted approximately 8 hours.

The area traverses were fully automated and were performed with the use of CarletonDAQ, previously described in Section 3.5.4. 


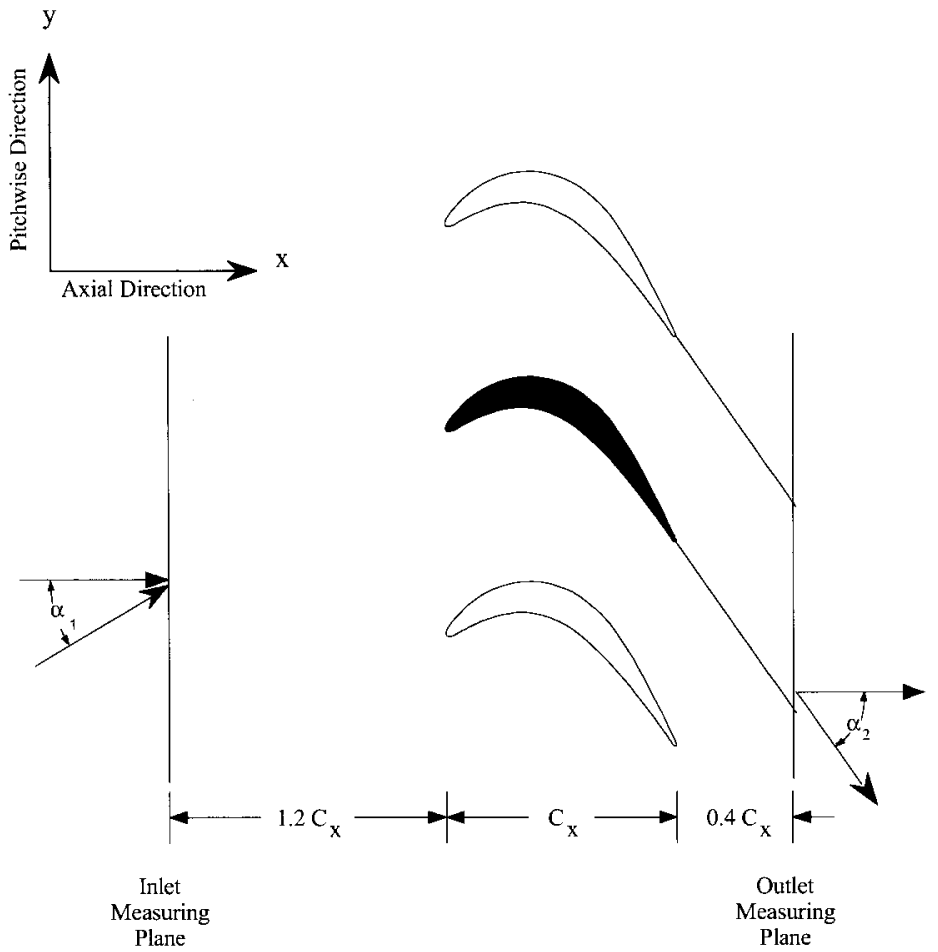

Figure 3.10: Measurement Plane

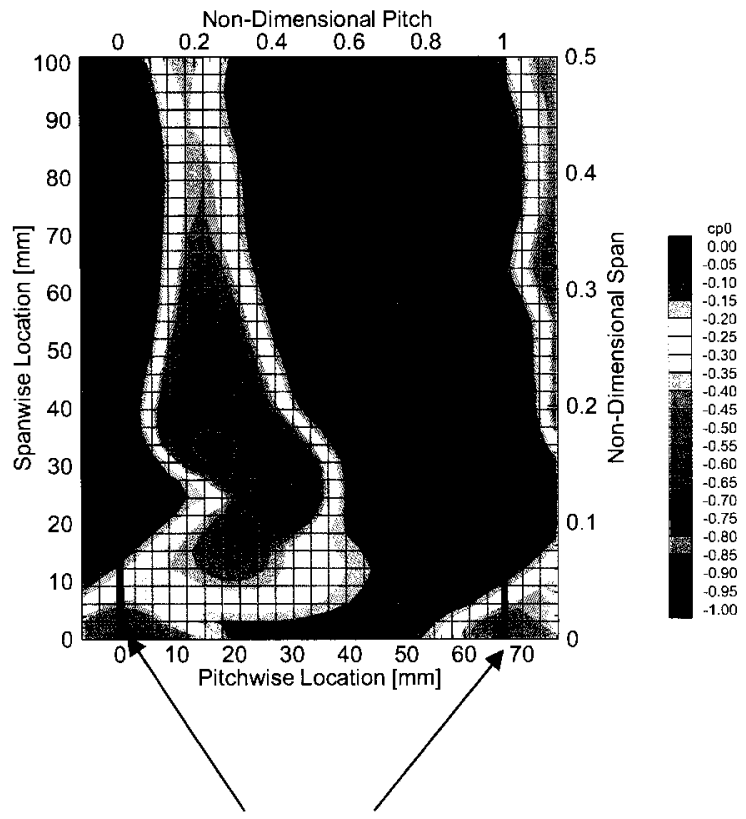

Trailing Edge Projection

Figure 3.11: Measurement Matrix 


\subsection{Data Reduction}

\subsubsection{Measured and Mixed-Out Loss Coefficients}

The total pressure loss, $Y_{T O T}$, discussed in Section 2.3, was defined as the sum of its individual components, namely: profile, tip-leakage, and secondary losses.

$$
Y_{T O T}=Y_{P}+Y_{T I P}+Y_{S E C}
$$

The present measurements were performed in the absence of tip clearance, thus the equation can be reduced to:

$$
Y_{T O T}=Y_{P}+Y_{S E C}
$$

The profile losses were not a part of the present investigation. Their integrated values were taken from Popovic (2005) whose measurements were done in conjunction with the present ones.

The total pressure losses were obtained from the seven-hole probe area traverses, as was already discussed in the previous section. The total pressure loss is given by the following equation:

$$
Y_{T O T}=\left(C_{P_{1}}{ }^{\prime \prime}-C_{P 0_{2}}{ }^{\prime \prime}\right)\left(\frac{q_{C L}}{q_{2}}\right)^{\prime \prime}
$$

where the mass-averaged total pressure loss coefficient, $C_{P 0}{ }^{\prime \prime}$, is defined in equation 3.5.

$$
C_{P 0}^{\prime \prime}=\frac{\iint \rho u\left(y^{\prime}, z\right) C_{P 0}\left(y^{\prime}, z\right) d y^{\prime} d z}{\iint \rho u\left(y^{\prime}, z\right) d y^{\prime} d z}
$$


and the total pressure coefficient at each measured location is:

$$
C_{P 0}=\frac{P_{0_{L O C A L}}-P_{0_{C L}}}{1 / 2 \rho V_{C L}^{2}}
$$

Based on the preceding equations, the secondary losses can be evaluated as:

$$
Y_{S E C}=Y_{T O T}-Y_{P}
$$

Note that the above equations are used to calculate the total pressure losses at the particular measurement plane. The measured losses are dependent on the location of the measurement plane since losses continue to be generated with downstream distance. Therefore, to be able to compare the results obtained from different cascades it is useful to determine the fully mixed-out losses. The mixed-out losses are evaluated at a hypothetical plane far downstream where the exit flow is fully mixed out and thus uniform (i.e. there is no further loss generation due to mixing). The mixing losses can be found by applying the momentum and continuity equations to a control volume extending from the measurement plane to the mixed-out plane. It is assumed that the mixing occurs over a constant area under incompressible conditions. The mixing analysis due to Harrison (1990), as adapted by Benner (2003), was used for the present measurements. Only the final equation for the mixed out losses is provided here, while the full derivation procedure can be found in Benner (2003).

$$
Y_{\text {TOT } T_{\text {mixed }}}=\left(C_{P 0_{1}}{ }^{\prime \prime}-C_{P 0_{\text {mixed }}}\right)\left(\frac{q_{C L}}{q_{\text {mixed }}}\right)^{\prime \prime}
$$

where $C_{P 0_{\text {mixed }}}$ and $q_{\text {mixed }}$ are the total and dynamic pressure at the mixed out plane, respectively. 
Estimated uncertainties in $Y_{S E C}$ were quoted in the respective sections. The method called Single-Sample Uncertainty Analysis due to Moffat (1982) was used. Due to the limited number of repeated calibrations, the uncertainties for pressure transducers and seven-hole probe measurements were estimated in part relying on uncertainties reported by Benner (2003) and Mahallati (2003), who over a number of years performed systematic calibrations of the equipment. The uncertainties in the profile losses were taken from Popovic (2005).

\subsubsection{Streamwise Vorticity}

The vorticity, $\omega$, given in equation 3.9 is defined as the curl of the velocity. In the physical world the vorticity can be thought of as the local component of rotation in a fluid flow.

$$
\omega=\nabla \times \mathbf{V}
$$

The streamwise vorticity is a projection of the total vorticity in a direction perpendicular to the flow. It is very useful in studying three-dimensional flows and its magnitude is a good indicator of the strength of the secondary flows. Data obtained from the seven-hole probe measurements was reduced using the method proposed by Gregory-Smith et al. (1988) based on the incompressible form of Euler equation. The axial and tangential components of the streamwise vorticity are given in equations 3.10 and 3.11 , respectively.

$$
\begin{gathered}
\omega_{x}=\frac{\partial w}{\partial y}-\frac{\partial v}{\partial z} \\
\omega_{y}=\frac{1}{u}\left(v \omega_{x}+\frac{1}{\rho} \frac{\partial P}{\partial x}\right)
\end{gathered}
$$


The streamwise vorticity is given by equation 3.12:

$$
\omega_{s}=\omega_{x} \cos \alpha^{\prime \prime}+\omega_{y} \sin \alpha^{\prime \prime}
$$

where the mass-averaged outlet flow angle can be calculated from:

$$
\alpha^{\prime \prime}=\left(\frac{\iint \rho u_{2} v_{2} d y^{\prime} d z}{\iint \rho u_{2}^{2} d y^{\prime} d z}\right)
$$

The non-dimensional form of vorticity is shown in equation 3.13 :

$$
C_{\omega_{s}}=\frac{\omega_{s} c}{V_{C L}}
$$

where $\mathrm{c}$ is the blade true chord and $V_{C L}$ is the velocity at inlet midspan (centerline).

Secondary circulation, $\Gamma_{s}$, represents the integrated streamwise vorticity and its magnitude provides information about the total streamwise vorticity present in a region of interest. It is defined in equation 3.14 .

$$
\Gamma_{s}=\int_{0}^{\frac{h}{2}} \int_{0}^{s} \omega_{s} d A_{s}
$$

where $d A_{s}$ is the differential area of a plane measured perpendicular to the streamwise vorticity defined as:

$$
d A_{s}=\cos \alpha_{2}^{\prime \prime} d y^{\prime} d z
$$

Non-dimensional form of the secondary circulation, $C_{\Gamma_{s}}$ is:

$$
C_{\Gamma_{s}}=\frac{\Gamma_{s}}{c V_{C L}}
$$


In addition to streamwise vorticity, the secondary kinetic energy (SKE) is another indicator of the strength of the secondary losses. It can be considered to be a source of loss in linear cascades, since it is completely lost through mixing processes. SKE is the kinetic energy associated with the velocity components in a plane perpendicular to the mass-averaged exit flow angle, $\alpha_{2}^{\prime \prime}$ and is given by equation 3.16 .

$$
S K E=\frac{1}{2} \rho\left(v_{S E C}^{2}+w_{S E C}^{2}\right)
$$

where

$$
v_{S E C}=-u_{2} \sin \alpha_{2}^{\prime \prime}+v_{2} \cos \alpha_{2}^{\prime \prime}
$$

and

$$
w_{S E C}=w_{2}
$$

The non-dimensional secondary kinetic energy is defined as:

$$
C_{S K E}=\frac{v_{S E C}^{2}+w_{S E C}^{2}}{V_{C L}^{2}}
$$

\subsubsection{Axial Velocity Ratio (AVR)}

The axial velocity-density ratio is an approximate measure of the streamtube convergence (or divergence) at the midspan. For linear cascade testing under incompressible condition, the density term is commonly omitted. Hence, the axial velocity ratio is defined as: 


$$
A V R=\frac{u_{2}^{\prime}}{u_{1}^{\prime}}
$$

where $u_{2}^{\prime}$ and $u_{1}^{\prime}$ are the midspan (centerline) mass-averaged axial velocity at the outlet and inlet, respectively.

A necessary but not sufficient condition for the flow to be two-dimensional is that AVR should be equal to 1.0. Values of AVR in excess of 1.0 indicate the existence of convergence in the flow in the spanwise direction (i.e. flow acceleration). Conversely, AVR values of less than 1.0 indicate that there is flow divergence in the spanwise direction. The term AVR is used in the present thesis in a slightly different, but related, way. The local value of AVR used here is defined as follows:

$$
A V R=\frac{u_{2 l o c a l}}{u^{\prime \prime}}
$$

where $u_{2 l o c a l}$ is the local axial velocity at the outlet, and $u^{\prime \prime}$ is the mass-averaged axial velocity. From conservation of mass and assuming the flow is symmetrical about the midspan plane, the mass-averaged value of AVR is 1.0 for the outlet plane. The local value of AVR thus indicates whether the local flow streamtube is deficient in mass flow $($ AVR $<1.0)$, or has excess mass flow (AVR $>1.0)$ compared with the average for the outlet plane. AVR will be further discussed in Chapters 4 and 5 .

This concludes the discussion pertaining to the experimental set-up, equipment and measurement procedures involved with the present research. The following chapters will focus on the results obtained from measurements at design and offdesign incidences. 


\section{Chapter 4}

\section{Secondary Flows at Design Incidence}

\subsection{Introduction}

The present work is a part of comprehensive ongoing research project on the PAKB and PAKD airfoils. Mahallati (2003), Dai (2004) and Popovic (2005) examined the midspan performance for the PAKB, PAKD-A, and PAKD-F airfoils, respectively. However, no studies up to this point have involved a detailed investigation into the secondary flows.

This chapter is concerned with the presentation and discussion of the results obtained from secondary flow measurements at a fixed Reynolds number of 80,000 and design incidence. The Reynolds number is based on the inlet velocity and blade axial chord (as discussed in Section 3.7.1), and the definition of the incidence is given in Section 2.2.1. 
The measurements for all three airfoils were performed at a plane located $0.4 C_{x}$ downstream of the blade trailing edges, as described in Section 3.7.3, and at two freestream turbulence intensities (FSTI) of $1.5 \%$ and $4.0 \%$.

Section 4.2 will introduce the midspan loadings for PAKB, PAKD-A, and PAKDF. In addition, it will compare the three airfoils using the quality factor, which is a measure of loading and its distribution, and its effects on secondary losses. The inlet flow will be discussed in Section 4.3. Section 4.4 will provide a detailed discussion of the measurements on the baseline airfoil, PAKB. The following section will compare the results of all three airfoils at the design incidence including the effect of varying FSTI on the secondary flows. Finally, the last section will summarize the results and conclusions.

\subsection{Blade Loading}

Figure 4.1 shows the midspan static pressure distributions around the airfoils (loadings) for PAKB, PAKD-A, and PAKD-F at the two investigated FSTI levels, $1.5 \%$ and $4.0 \%$. The loading is directly related to the tangential force applied by the airfoil. The tangential force is equal to the area enclosed by two curves representing the static pressure distribution on the suction surface (upper curve) and that on the pressure surface (lower curve); the larger the area between the curves, the greater the magnitude of the tangential force.

PAKD-A and PAKD-F have 25\% higher loading than the baseline airfoil, PAKB. Hence the area enclosed between the curves is greater in their cases as seen in Figure 4.1. PAKB and PAKD-A are aft-loaded, meaning that the resultant tangential force is acting towards the aft part of the airfoil. The location of their suction peaks (the 


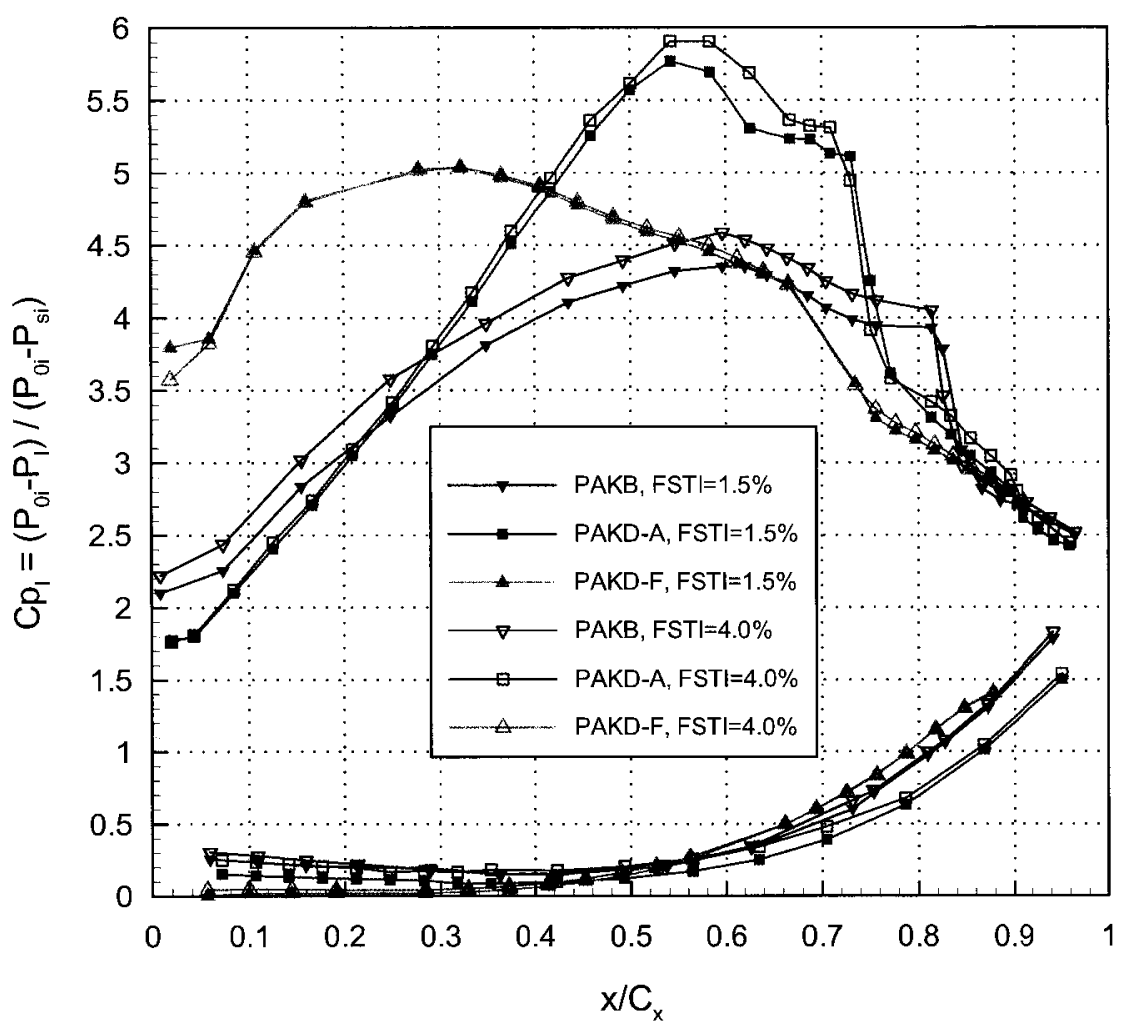

Figure 4.1: Blade Loadings at Design Incidence $(R e=80,000, F S T I=1.5 \%$ and $4.0 \%)$

point with highest value of local pressure coefficient), is at about 0.61 and $0.55 C_{x}$ for PAKB and PAKD-A, respectively. The PAKD-F, by comparison, is forward-loaded and its suction peak is at approximately $0.32 C_{x}$.

The loading has multiple effects on the secondary flow. Not only do the absolute loading levels affect the secondary flows, but the distributions of loading have a significant influence as well. Dossena et al. (2004), Weiss and Fottner (1995), Hodson and Dominy (1987a) and Perdichizzi and Dossena (1993) investigated the effect of loading and its distribution on the secondary flows and losses. All agree that more highly loaded blades in general exhibit stronger secondary flows and increased 
losses due to them. In addition, they also found that forward-loaded airfoils showed increased secondary flows and losses, compared to their aft-loaded counterparts.

Marchal (1980) proposed the quality factor, Q, defined by equation 4.1, as a measure of the blade loading and loading distribution.

$$
Q=\left(\Delta C_{P \max } \frac{q_{C L}^{\prime \prime}}{q_{\text {mean }}^{\prime \prime}} \frac{C_{x}}{s^{*}}\right)^{1.25}\left(1-\frac{X_{\min }}{C_{x}}\right)
$$

where $\Delta C_{P \max }$ is the maximum dimensionless cross-passage pressure difference and $q_{C L}^{\prime \prime}$ is the mass-averaged dynamic pressure at the inlet centerline. $q_{m e a n}^{\prime \prime}$ is the vector mean of the inlet and outlet mass-averaged dynamic pressures at the centerline. $s^{*}$ is the cross-passage distance between suction and pressure surface of two adjacent airfoils at $X_{m i n}$, where $X_{m i n}$ represents the axial location of the suction peak measured from the leading edge. The relationship between the quality factor and the secondary losses will be investigated for the present three cascades.

Marchal (1980) is of the opinion that the higher cross-passage pressure difference (higher loading) or a shift in loading distribution toward the front of the airfoil would increase the secondary losses. He argued that the higher the value of $\mathrm{Q}$ of the particular airfoil, the higher the secondary loss. Weiss and Fottner (1995) and Benner et al. (2004a) confirmed this in their investigations. The present data, as will be seen in Table 4.1, indicates that the forward-loaded blade, PAKD-F, is expected to have the highest losses, followed by the aft-loaded PAKD-A and the baseline airfoil, PAKB.

The differences in the quality factor between two FSTI levels comes from slightly different loadings $\left(\Delta C_{P \max }\right)$, as can be seen in Figure 4.1 , and values of the dynamic 
Table 4.1: Quality Factor Summary for the Three Cascades

\begin{tabular}{|l|c|c|c|}
\cline { 2 - 4 } \multicolumn{1}{c|}{} & PAKB & PAKD-A & PAKD-F \\
\hline FSTI $=1.5 \%$ & 4.4 & 5.9 & 6.6 \\
\hline FSTI $=4.0 \%$ & 4.7 & 6.1 & 6.7 \\
\hline
\end{tabular}

pressure.

The presence of a separation bubble on the airfoil surface may also affect the secondary flow and losses. Separation bubbles can be identified by the plateau in the local pressure distribution. As seen from Figure 4.1, the separation points on the suction surfaces of PAKB and PAKD-A airfoils are at approximately 0.72 and $0.62 C_{x}$ followed by reattachment at about 0.84 and $0.76 C_{x}$, respectively. No suction side separation bubble was detected by Popovic (2005) for PAKD-F at design conditions. In addition, Mahallati (2003) identified a separation bubble near the leading edge on the pressure side of the PAKB airfoil at design incidence. No separations on the pressure surfaces of the PAKD airfoils were detected by Dai (2004) and Popovic (2005). Duden and Fottner (1999) argue that as a result of the suction surface laminar separation bubble an increase in losses in the endwall area (secondary losses) can occur. Brear et al. (2002) investigated the interaction between the pressure surface separation bubble and the secondary flow. They found that this interaction can significantly affect the strength of the secondary flow and the magnitude of the secondary losses. The effect of the PAKB pressure surface separation bubble on secondary flows will be discussed in Section 4.5 . 


\subsection{Inlet Endwall Boundary Layer}

The inlet boundary layer traverses were taken with a Pitot probe at a location of approximately $1.2 C_{x}$ upstream of the middle-blade leading edge. Each traverse extended from the turntable endwall to the blade half span. The static pressure was obtained from the static tap located on the plexiglass endwall at the same location (i.e. $1.2 C_{x}$ upstream of the middle blade). The velocity profiles were calculated from the total and static pressure measurements. The obtained velocities were normalized by the centerline velocity, $V_{C L}$.

Figure 4.2 shows the normalized velocity profile $\left(V / V_{C L}\right)$ at design incidence

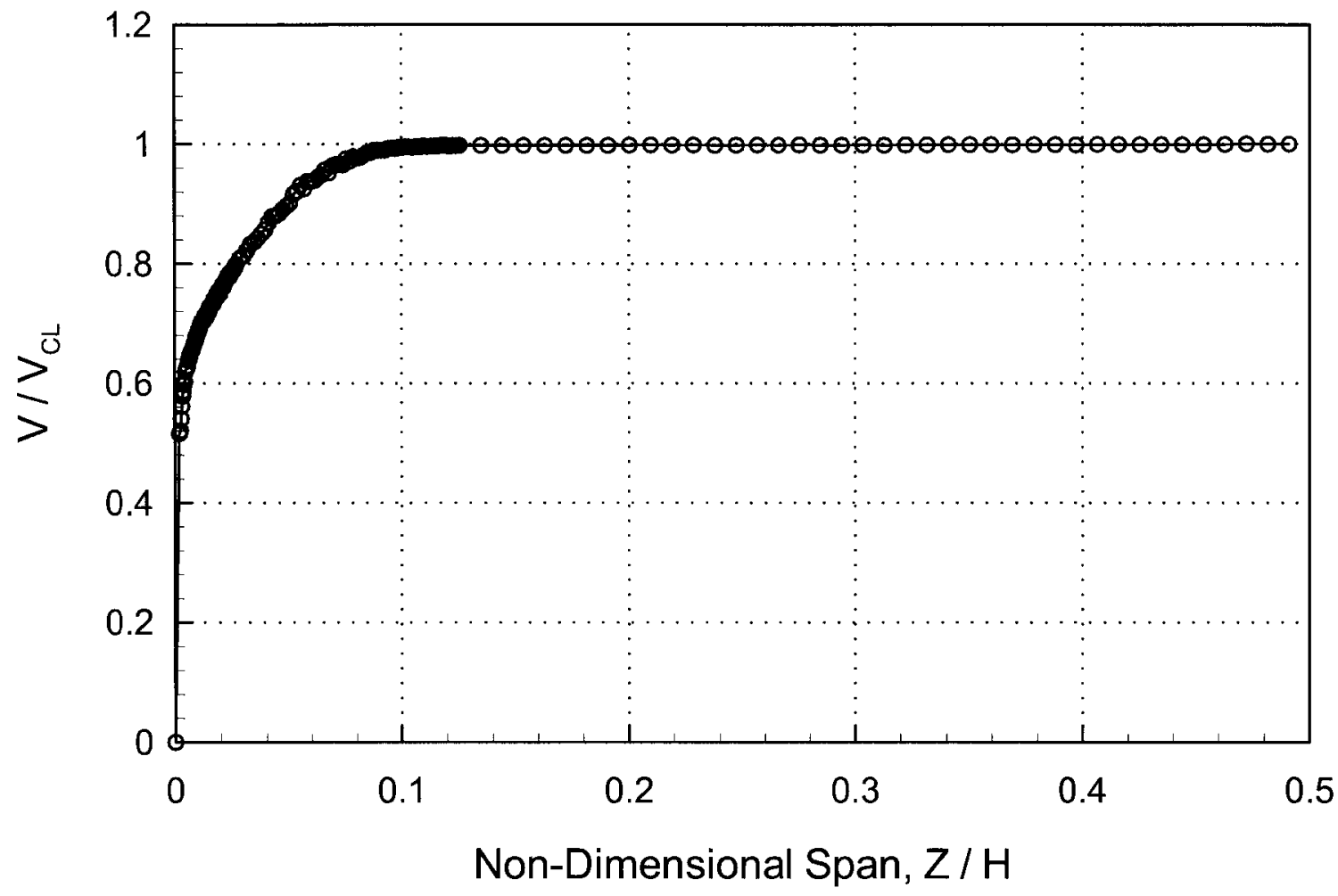

Figure 4.2: Inlet Velocity Profile - No Grid Installed, FSTI $=\mathbf{0 . 4 \%}$ 
and with no grid installed (freestream turbulence intensity, FSTI=0.4\%). The flow exhibits, as seen in the figure, excellent uniformity in the freestream (away from the endwall).

However, this low level of FSTI is not representative of the engine environment and in order to increase the FSTI levels to those more comparable of a real engine environment (3-7\%, private communications with PWA, East Hartford, CT, 2004), turbulence generating grids were utilized, as mentioned in Section 3.4. Figures 4.3 and 4.4 show normalized velocity profiles at three pitchwise locations for FSTI levels of $1.5 \%$ and $4.0 \%$, respectively.

As seen in Figures 4.3 and 4.4, increased turbulence levels cause non-uniformities in the inlet flow in both the pitchwise and spanwise directions. To account for these variations it was decided that instead of basing the inlet total pressure coefficient $\left(C_{P 01}^{\prime \prime}\right.$, defined in Section 3.8.1) on one traverse (as could be done in case of the uniform inlet flow in pitchwise direction) it would be based on an area traverse of the inlet flow covering the estimated streamtube that enters the passage. The measurements were performed using the seven-hole pressure probe on a grid spaced $3.0 \%$ and $2.4 \%$ of the blade pitch and for PAKB and PAKD airfoils, respectively, as discussed in Section 3.7.3.

Integrated values of the total pressure coefficients and inlet boundary layer parameters are shown in Tables 4.2 and 4.3 , respectively.

As can be seen from Table 4.3 the boundary layer thicknesses are different for the two FSTI levels. The boundary layer is almost twice as thick when the lower FSTI grid is employed. This is mainly due to the fact that the $1.5 \%$ turbulence generating grid is installed farther upstream of the test cascades and the boundary layer has a 


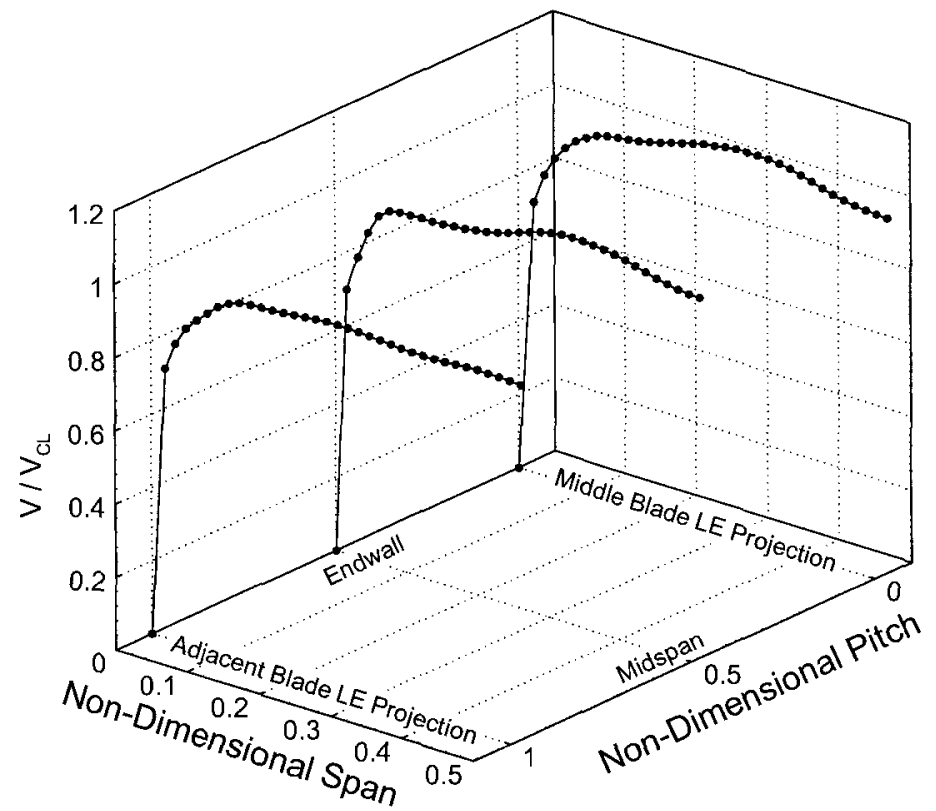

Figure 4.3: Inlet Velocity Profile, FSTI $=\mathbf{1 . 5 \%}$

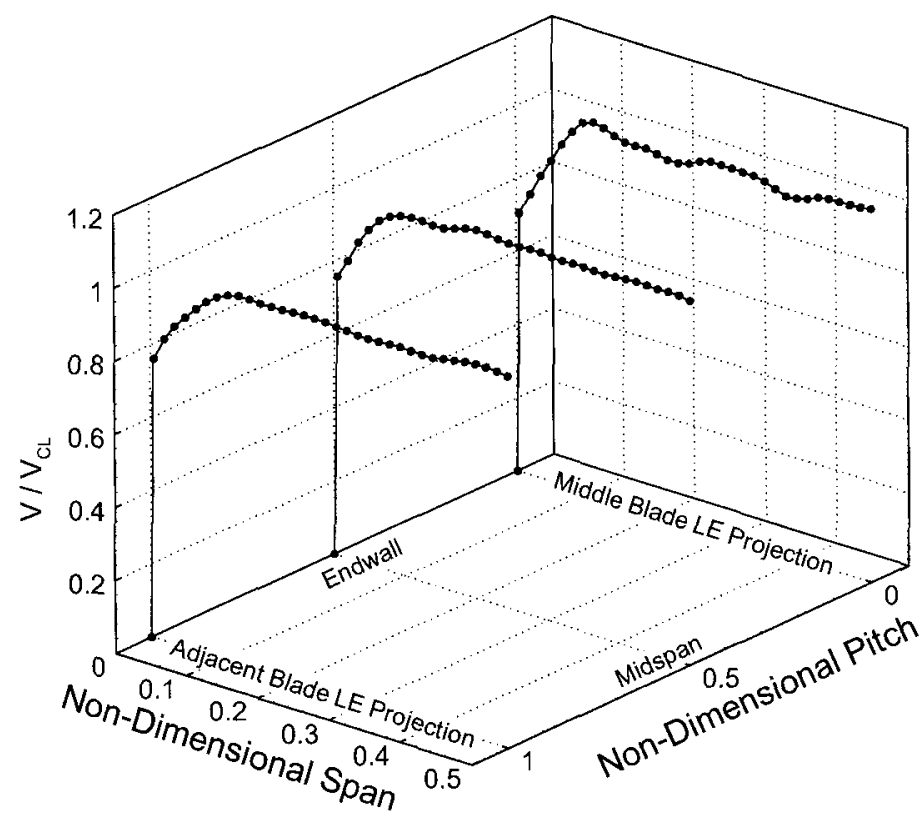

Figure 4.4: Inlet Velocity Profile, FSTI $=4.0 \%$ 
longer distance over which it can develop (as can be seen in Figure 3.3, Section 3.3).

Table 4.2: Inlet Total Pressure Coefficients at Design Incidence for FSTI of $\mathbf{1 . 5 \%}$ and $4.0 \%$

\begin{tabular}{|c|c|c|c|c|}
\cline { 3 - 5 } \multicolumn{1}{c|}{} & PAKB & PAKD-A & PAKD-F \\
\hline \multirow{2}{*}{$\mathrm{C}_{\mathrm{PO}} "$} & $\mathrm{FSTI}=1.5 \%$ & -0.017 & -0.012 & -0.012 \\
\cline { 2 - 5 } & $\mathrm{FSTI}=4.0 \%$ & -0.036 & -0.035 & -0.035 \\
\hline
\end{tabular}

Table 4.3: Summary of Inlet Boundary Layer Parameters at Design Incidence for FSTI of $1.5 \%$ and $4.0 \%$

\begin{tabular}{|c|c|c|}
\cline { 2 - 3 } \multicolumn{1}{c|}{} & $\mathrm{FSTI}=1.5 \%$ & $\mathrm{FSTI}=4.0 \%$ \\
\hline$\delta / \mathrm{h}$ & 0.18 & 0.10 \\
\hline$\delta^{*}[\mathrm{~mm}]$ & 4.0 & 1.4 \\
\hline$\theta[\mathrm{mm}]$ & 2.9 & 1.1 \\
\hline $\mathbf{H}_{\mathrm{SF}}$ & 1.4 & 1.3 \\
\hline
\end{tabular}

The $\delta / h$ shown in Table 4.3 represents the obtained boundary layer thickness non-dimensionalized by the blade height ( $\operatorname{span).~} \delta^{*}$ is boundary layer displacement thickness and $\theta$ is the momentum thickness. $H_{S F}$ is the shape factor.

The influence of the inlet boundary layer (IBL) thickness on the endwall flow in turbines has been investigated by several researchers. In his literature review, Dunham (1970) summarized previous investigations on secondary flow phenomena and concluded that secondary losses depend on endwall inlet boundary layer, exhibiting the largest sensitivity when boundary layers are very thin. Other researchers (Came (1973), Sauer and Wolf (1994)) reported an increase in secondary losses with increasing boundary layer thickness until a certain "critical" value after which there 
was no further dependence. Chen and Dixon (1986) varied the endwall boundary layer thickness in a linear cascade experiment and concluded that the secondary loss coefficient, $Y_{S E C}$ (defined in Section 2.3), does not vary significantly with IBL thickness. Hodson and Dominy (1987b) also did not find a relationship between the inlet boundary layer thickness and secondary losses. A similar study was made by Gregory-Smith et al. (1988), who measured the flow in a linear cascade for three different IBL thicknesses. They found no clear trends in the secondary losses.

Although the general view is that the inlet boundary layer thickness does not affect the secondary flows greatly, further investigations are needed to provide more insight into the variation of secondary losses with IBL thickness. More specifically, it is recommended that such study should attempt to determine whether there is, as some researchers suggest, the "critical" value of the IBL thickness after which the secondary losses become fairly insensitive to the changes in IBL thickness. The influence of the the inlet boundary layer thickness on the secondary losses obtained in the present study will be further discussed in Section 4.4.3.

\subsection{Detailed Discussion of the Baseline Airfoil}

\subsubsection{Total Pressure and Vorticity Distributions}

This section will introduce the total pressure and streamwise vorticity distributions measured downstream of the blade trailing edges. Streamwise vorticity was introduced in Section 3.8.2. Its magnitude is a good indicator of the secondary flow strength.

The total pressure coefficient $\left(C_{P 0}\right)$ contours as measured at $0.4 C_{x}$ downstream of 
the blade trailing edges for PAKB at design incidence and FSTI of $1.5 \%$ are shown in Figure 4.5. The plot on the left shows the projections of the blade trailing edges to the measurement plane and indicates the suction and pressure sides of the passage. As shown, the plots extend from the endwall to the midspan of the blade passage. A rectangle covering half span and $1.2 \mathrm{~s}$ (blade pitches) was traversed. The point density was approximately $3.6 \%$ and $4.5 \%$ of the blade pitch for PAKB and PAKD airfoils, respectively. A more detailed description of measuring procedures and measurement matrix was given in Section 3.7.3. The right hand side of the Figure 4.5 shows the flood contours of total pressure coefficient with the main vortical structures associated with the secondary flow as inferred from the measurements. The darker blue shades indicate higher total pressure deficit (i.e. higher losses), while the red regions are the areas of the "loss free" fluid. The direction of rotation of the vortices was determined from the sign of the streamwise vorticity as obtained from the measurements, as described below.

The total pressure contours shown here exhibit the typical behavior of flows in the endwall regions of turbines. The areas of high total pressure loss coincide with the areas of greatest magnitudes of streamwise vorticity. Figures 4.6 and 4.7 show the positive and the negative vorticity distributions for PAKB at FSTI=1.5\%, respectively, with superimposed line contours of $C_{P 0}$.

Three major vortical structures, as discussed in Section 2.4, can be identified in Figures 4.6 and 4.7. The passage vortex, whose vorticity has a negative sign, is rotating clockwise and is the most dominating feature seen in Figure 4.7. It is located close to the endwall. The counter vortex is located closer to the suction surface and more towards midspan. It is rotating counterclockwise and thus has a positive streamwise vorticity, as seen in Figure 4.6. The counter vortex occupies an 

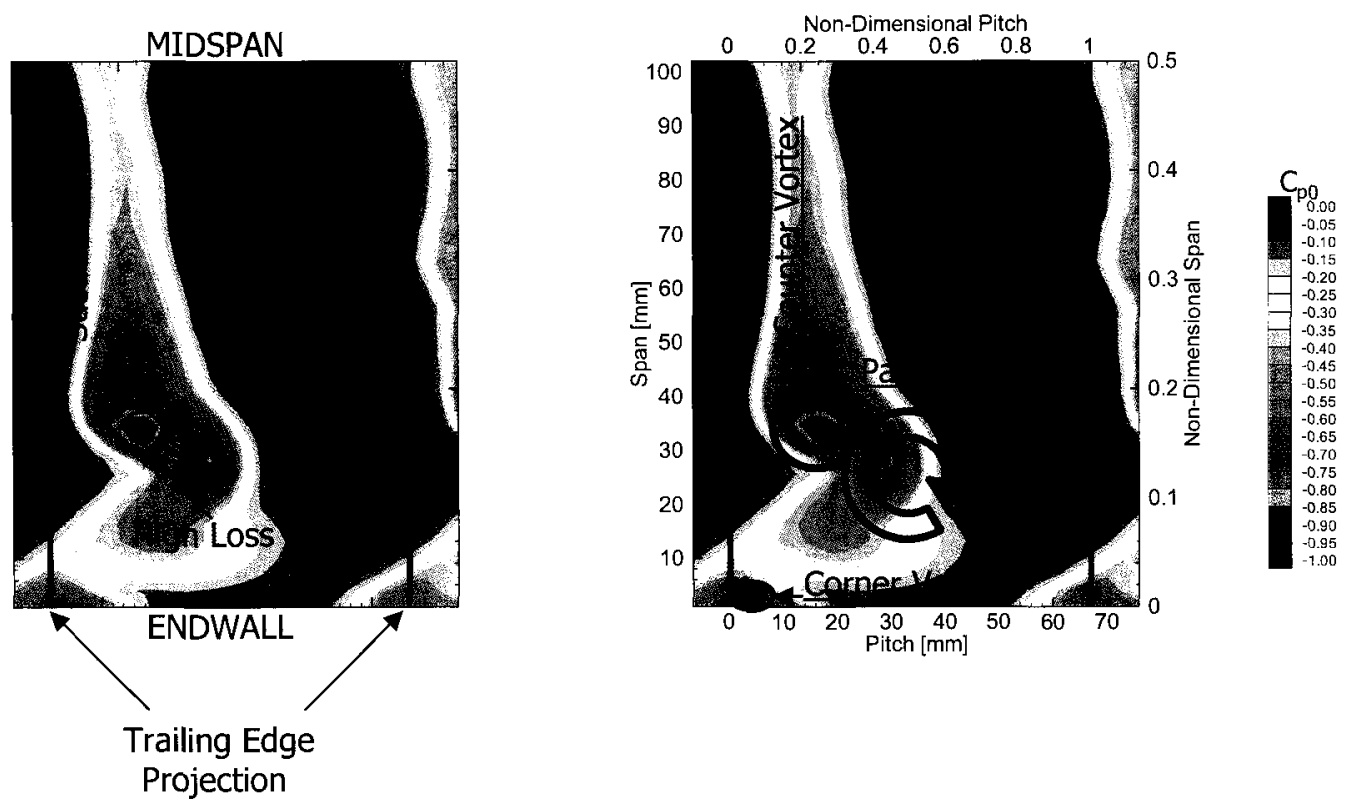

Figure 4.5: PAKB - Measurement Plane and Total Pressure Contours at $\mathrm{FSTI}=1.5 \%$

area smaller than that of the passage vortex, but the peak magnitude of its vorticity is greater. Lastly, a corner vortex, nested between the suction surface and endwall having the same sense of rotation as the counter vortex can be identified in Figure 4.6.

\subsubsection{Axial Velocity Ratio (AVR) Distribution}

The AVR and its definition was discussed in greater detail in Section 3.8.3.

Figure 4.8 shows the contours of axial velocity ratios (AVR) in the measurement plane (i.e. $0.4 C_{x}$ downstream of the blade trailing edge). As can be seen in the figure, there are three distinctive regions present. First, there is a region near the midspan 


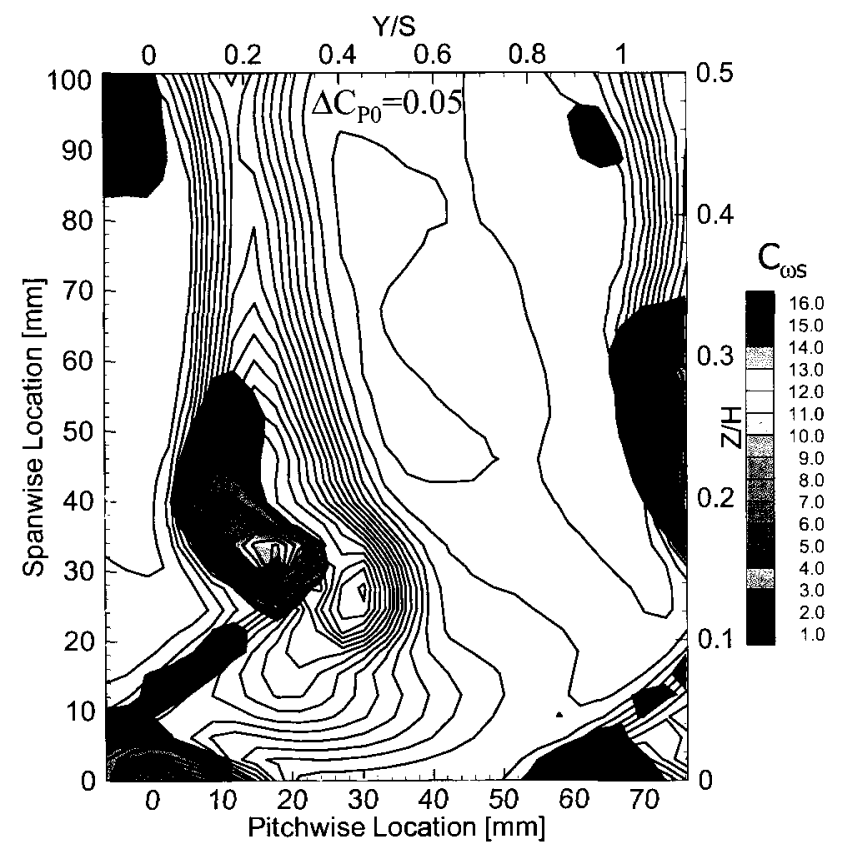

Figure 4.6: PAKB - Line Contours of Total Pressure Coefficient Superimposed on Flood Contours of Positive Vorticity at FSTI $=1.5 \%$

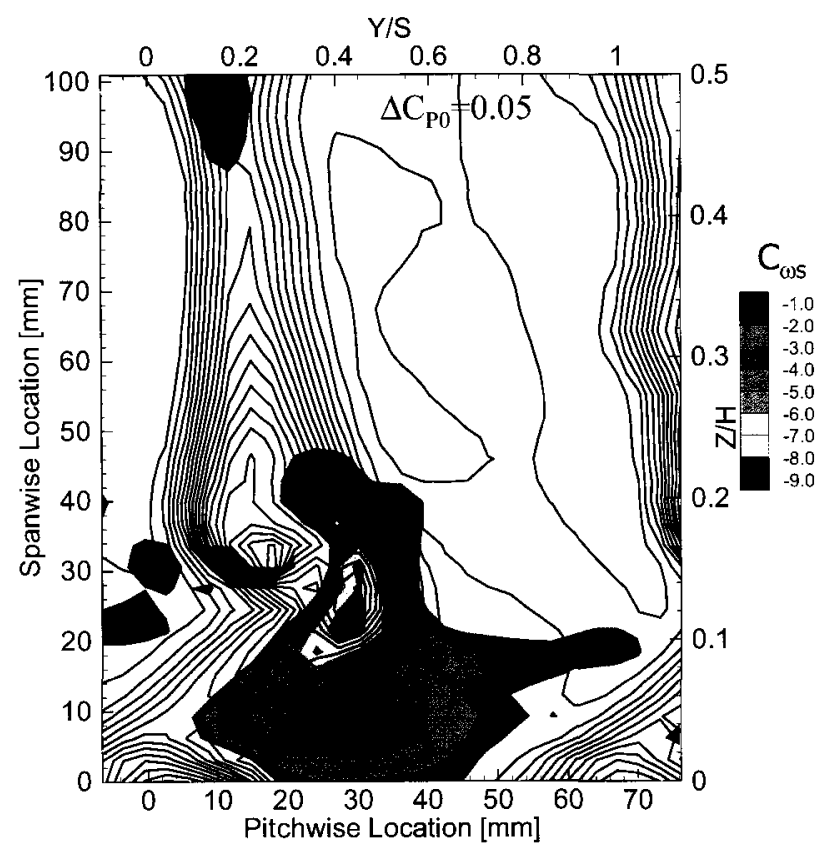

Figure 4.7: PAKB - Line Contours of Total Pressure Coefficient Superimposed on Flood Contours of Negative Vorticity at FSTI $=1.5 \%$ 
with AVR values of about 1.0, secondly there is a region of low AVR that is associated with boundary layer near the endwall, the wake and the vortical structures, and lastly a region of high AVR, with values well in excess of 1.0 that is nestled between the midspan and the endwall. Of particular interest is the region where the AVR reaches about 1.3. This patch of high axial velocity (overspeed) is not associated with high loss, and is nested between the three vortices, namely the passage, the counter and the corner vortex and the extension of the suction surface. To the author's knowledge, no previous studies have reported similar findings.

Due to the lack of flow visualization or detailed measurements within the blade passage, it is impossible to be sure of the origins of this AVR overshoot, but it appears

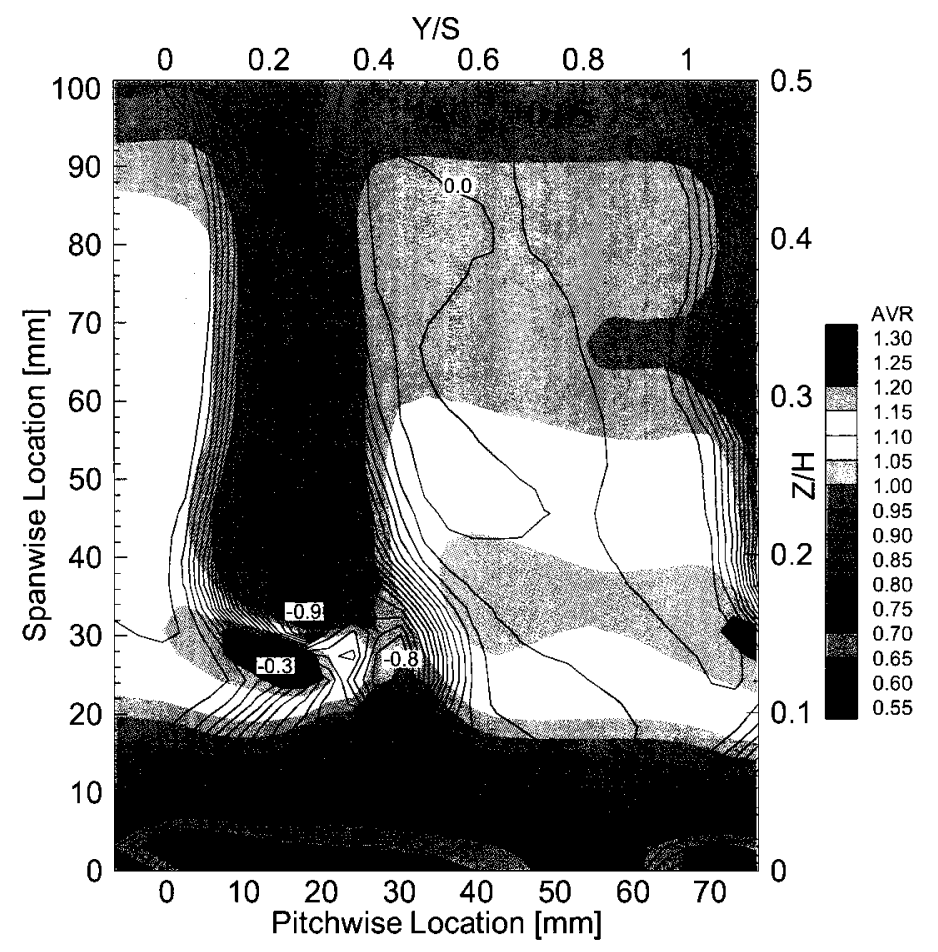

Figure 4.8: PAKB - Line Contours of Total Pressure Coefficient Superimposed on Flood Contours of Axial Velocity Ratio at FSTI $=1.5 \%$ 
that a region of relatively loss free fluid is funnelled between the two larger counterrotating vortices, the corner vortex and the blade trailing edge, and overspeeds because it is forced through a contracting stream tube. The origin of this overspeed fluid should be investigated further by additional traverses located in the blade passage and closer to the trailing edge.

The overspeed was also detected for PAKD-A and PAKD-F airfoils, as will be discussed in Section 4.5.2.

\subsubsection{The Effect of Varying Freestream Turbulence Intensity}

The effect of varying freestream turbulence intensity (FSTI) on the secondary losses have been examined by a number of investigators in the past. After testing at three FSTI levels $(0.8 \%, 2.2 \%$, and 3.8\%) Adjlout and Dixon (1992) concluded that the net secondary loss does not change significantly with freestream turbulence intensity. Gregory-Smith and Cleak (1992) found that when they introduced a turbulence generating grid into the previously clean wind tunnel flow, thus raising the FSTI levels to about $5.0 \%$, the secondary losses reduced by approximately $22 \%$. They believed that this was due to the thinner inlet boundary layer at the elevated FSTI level. However, this argument somewhat contradicts those already discussed in Section 4.3, where it was concluded that secondary losses were not greatly dependent on the inlet boundary layer thickness.

The present measurements were performed at FSTI levels of $1.5 \%$ and $4.0 \%$. Figure 4.9 shows the total pressure contours for PAKB at the two FSTI levels. With increased FSTI (showed on the right hand side of Figure 4.9) the area of the flow influenced by secondary flows is pushed closer to the projection of the trailing edge 
on the suction side of the passage. It is also displaced slightly further away from the endwall. However, the mixed-out mass-averaged secondary loss coefficient was found to be lower at higher FSTI: $Y_{S E C}=0.035$ and $Y_{S E C}=0.028$ for FSTI of $1.5 \%$ and $4.0 \%$, respectively.
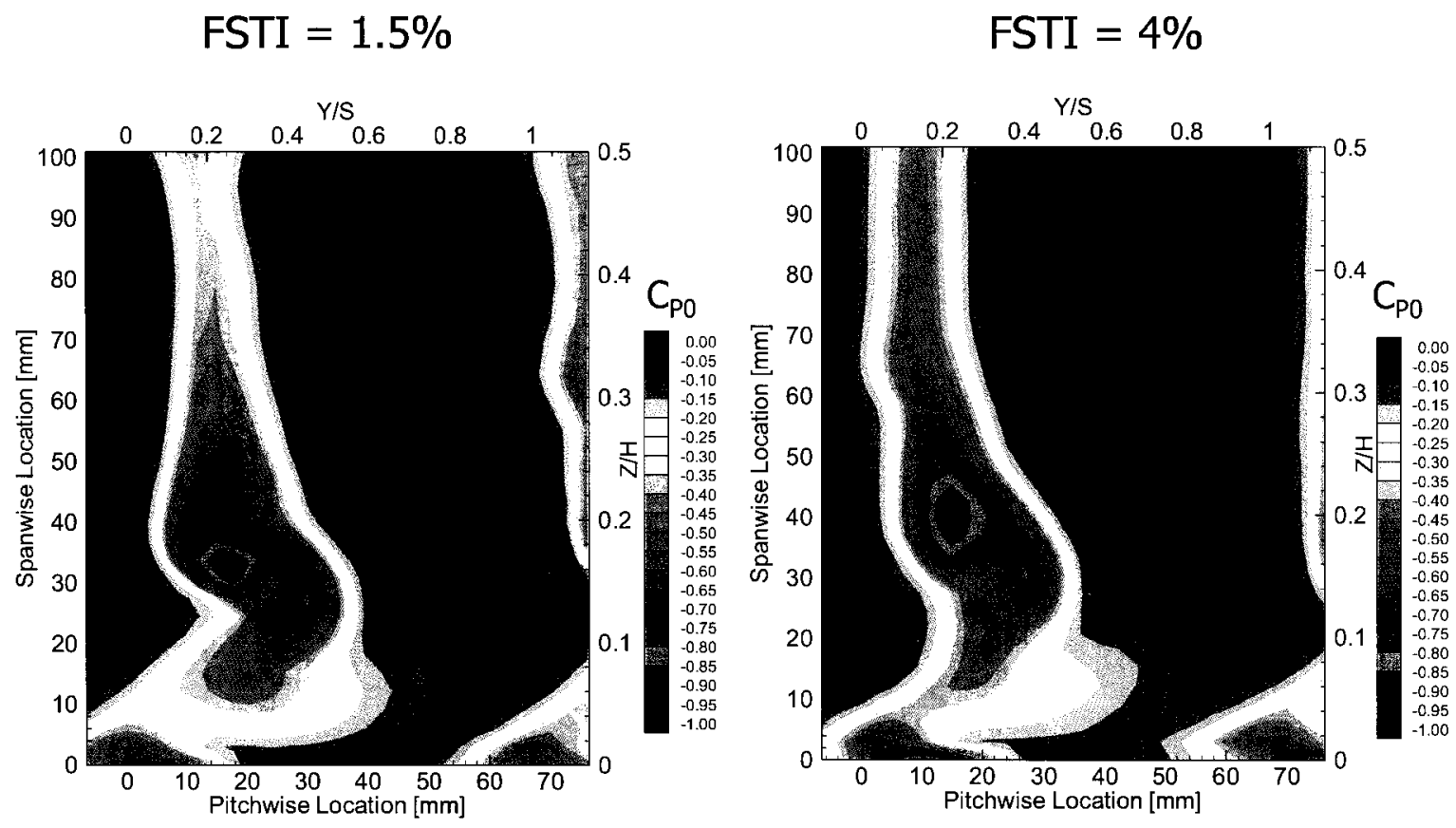

Figure 4.9: PAKB - Total Pressure Contours at FSTI=1.5\% and $4.0 \%$

Increased FSTI also has an effect on the streamwise vorticity. This is visible in Figures 4.10 and 4.11 which show the positive and negative vorticity for PAKB at the two FSTI levels, respectively. At both FSTI levels the peak magnitude of both positive and negative vorticity decreases with the increase in the FSTI. The magnitude of the peak positive vorticity is reduced, but the extent is enlarged. As summarized in Table 4.4, increased extent of the region of positive vorticity also leads to a small increase in the positive secondary circulation. The secondary circulation (as discussed in Section 3.8.2) represents integrated vorticity and is a good indicator of the strength 
of the secondary flow. The area of the flow under the influence of negative vorticity, which is associated with the passage vortex, has decreased with elevation of FSTI. As in the case of the positive vorticity, the peak magnitude of the negative vorticity was considerably decreased at higher FSTI. However, in the case of negative vorticity there was a reduction in integrated values of negative circulation. Secondary kinetic energy is another indicator of the secondary flow strength. Its mass-averaged values also show a reduction in magnitude at higher FSTI.

\section{Table 4.4: PAKB: Secondary Losses and Circulation}

\begin{tabular}{|l|c|c|}
\cline { 2 - 3 } \multicolumn{1}{c|}{} & FSTI=1.5\% & FSTI $=4.0 \%$ \\
\hline$Y_{\text {SEC }}$ & 0.035 & 0.028 \\
\hline$C_{\Gamma+}$ & 0.328 & 0.338 \\
\hline$C_{\Gamma-}$ & -0.260 & -0.198 \\
\hline$C_{\text {SKE }} "$ & 0.020 & 0.015 \\
\hline
\end{tabular}

Section 4.3 discussed the effect of inlet boundary layer (IBL) thickness on secondary losses. As mentioned, the IBL for the cases with FSTI of $4 \%$ is significantly thinner than the ones for the lower FSTI measurements. Therefore, a possible coupled effect of IBL and FSTI on the secondary losses cannot be ruled out. The author suggests that it would be worthwhile looking experimentally at the independent effects of FSTI and IBL on secondary flows.

This section dealt with secondary flow for the baseline airfoil, PAKB. The following section will compare the baseline blade with the two more highly loaded ones; PAKD$\mathrm{A}$ and PAKD-F. 

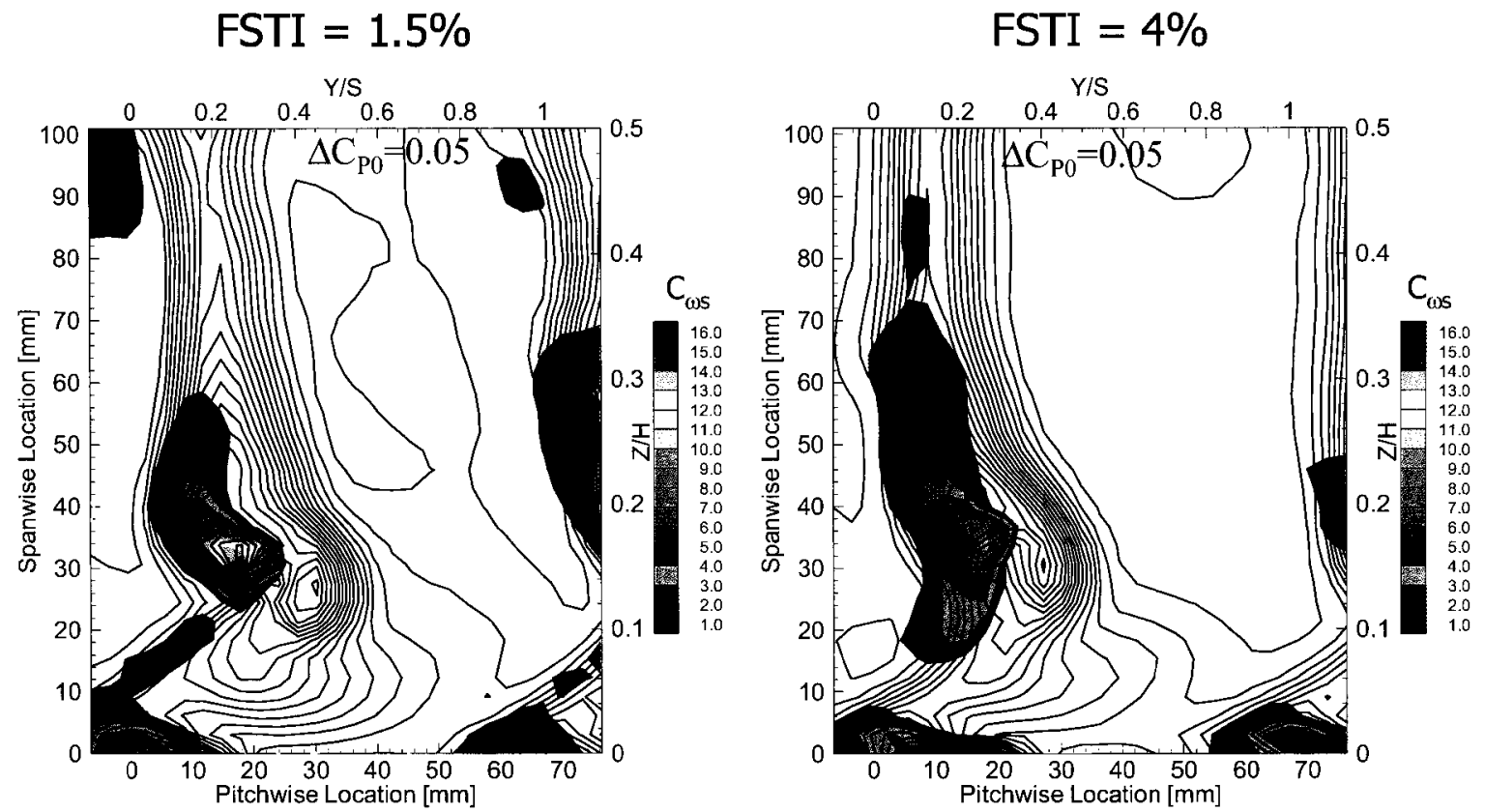

Figure 4.10: PAKB - Line Contours of Total Pressure Coefficient Superimposed on Flood Contours of Positive Vorticity at FSTI $=1.5 \%$ and $4.0 \%$
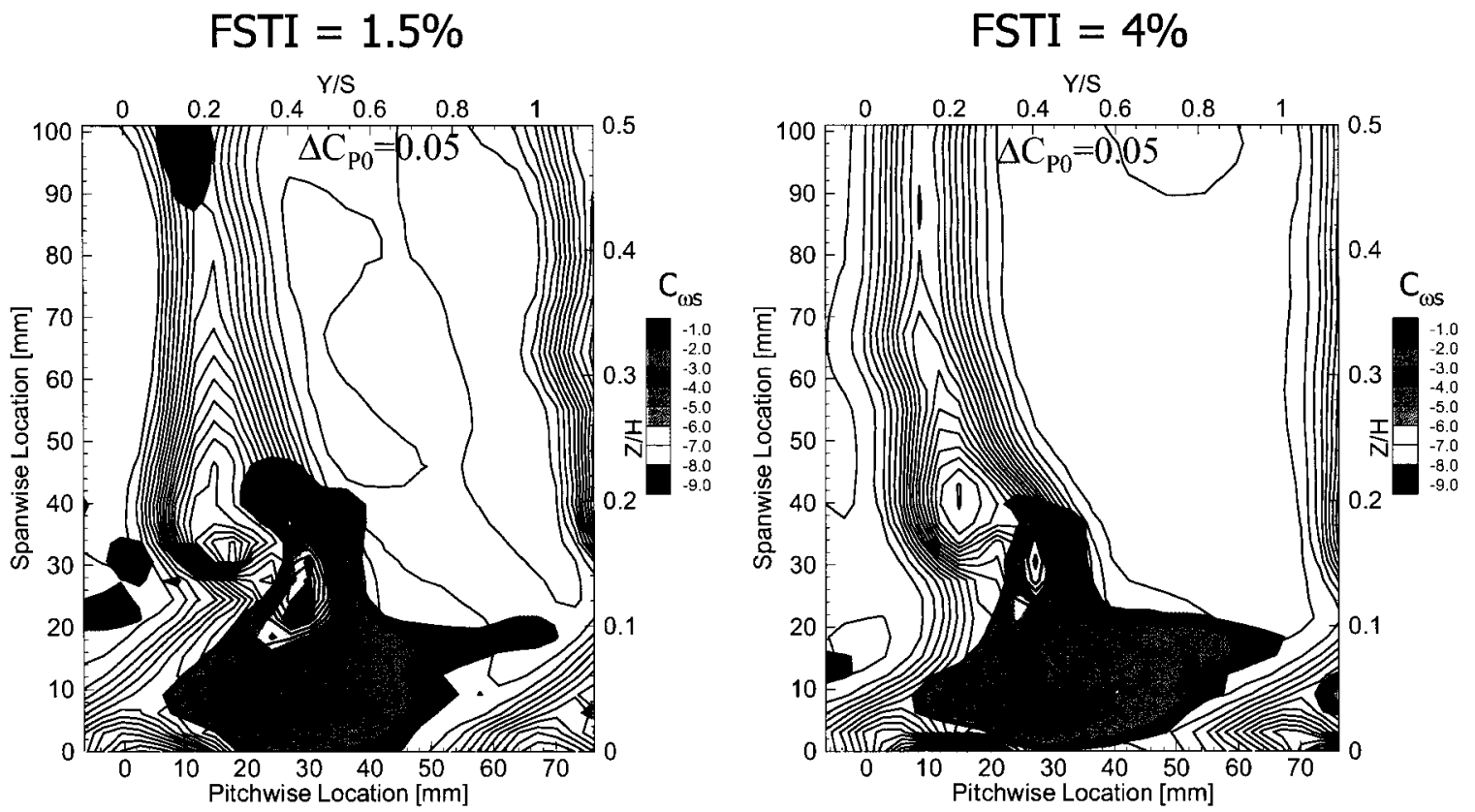

Figure 4.11: PAKB - Line Contours of Total Pressure Coefficient Superimposed on Flood Contours of Negative Vorticity at FSTI $=1.5 \%$ and $4.0 \%$ 


\subsection{Comparison of Results for All Three Cascades}

\subsubsection{Total Pressure and Vorticity Distributions}

Contours of the total pressure coefficients for all three cascades at FSTI of $1.5 \%$ are shown in Figure 4.12. The general features of the $C_{P 0}$ contours for all three blades look similar. However, contrary to the two loss peaks seen for PAKB, measurements for two PAKD blades clearly show there is a third one that is, as will be seen in Figures 4.13 and 4.14, not associated with strong vorticity. Popovic (2005) found that the PAKD-A blade is on the verge of stall at $R e=80,000$. Therefore, it is believed that the third loss peak seen in the PAKD-A contours might be a result of the onset of two-dimensional separation, perhaps triggered by the interaction of the counter vortex with the suction surface boundary layer. However, it is not clear what the source of the similar structure for PAKD-F is. From his midspan measurements, Popovic (2005) concluded that the PAKD-F airfoil does not have a separation bubble on the midspan of the suction side of this airfoil at $R e=80,000$. It is however possible that the cause of the third loss peak at about $25 \%$ of the blade span of PAKD-F might be again a local two-dimensional separation, as it is the case for PAKD-A, but further tests are required to verify this statement. Section 4.5 .3 will discuss further the question of the third loss peak for both PAKD airfoils.

Other differences between the total pressure distributions for the three blades are the pitchwise and spanwise extent of the secondary flow regions. The PAKB and PAKD-F secondary flow regions are slightly further from the endwall, and they extend somewhat further in the pitchwise direction, compared with the passage width. The PAKD-A contours are more "stretched" in the spanwise direction and have less of the "bone-like" shape of the contours for the other two airfoils. 

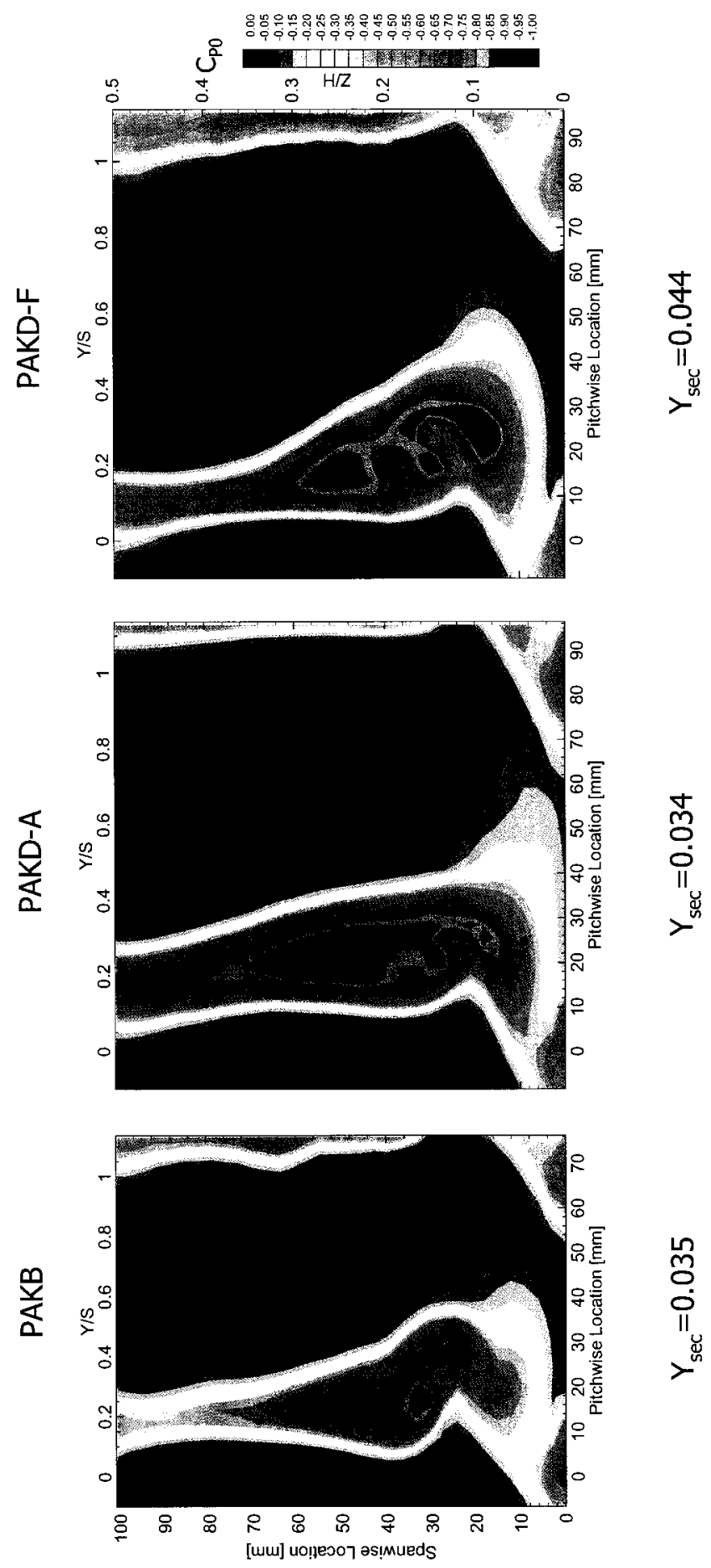

Figure 4.12: Total Pressure Loss Contours - Comparison of the Three Airfoils at $\mathrm{FSTI}=1.5 \%$ 
Section 4.2 introduced the quality factor and gave a summary of the values for the three investigated airfoils. Based on the quality factor and the observations of previous researchers, it was expected that the PAKD-F airfoil would have the strongest secondary flows and consequently the highest magnitude of losses, PAKDA would follow, and PAKB would have the weakest secondary flow and lowest losses. The integrated values of mass-averaged mixed-out loss coefficients, secondary circulation, secondary kinetic energy and quality factors for all three airfoils are given in Table 4.5. The PAKD-F airfoil indeed has the highest losses, as expected based on the quality factor. However, PAKB and PAKD-A losses are comparable. These results were not expected based on the values of quality factor which predicts higher secondary losses for the PAKD-A airfoil. In addition, visual inspection of the total pressure loss contours shown in Figure 4.12 suggest higher losses for PAKD-A. Upon examination of the results presented in Table 4.5 one can see that total losses are indeed highest for the PAKD-A airfoil. As mentioned previously, the PAKD-A is on the verge of stall under these conditions, thus the profile losses are higher than for the other two airfoils, and as a result, the magnitude of the secondary losses is reduced. As will be shown in Figure 4.15 in the next section, the region of high total pressure loss coincides with a region of relatively low axial velocity, thus the contribution of the losses in this area to the overall mass-averaged total loss is decreased. Values of mass-averaged secondary kinetic energy $\left(C_{S K E}^{\prime \prime}\right)$ also show comparable magnitudes for the PAKB and PAKD-A blades, whereas PAKD-F has higher $C_{S K E}^{\prime \prime}$. Summary of mass-averaged experimental results for all three airfoils at design incidence will be given in Appendix D.

The positive and negative vorticities are plotted in Figures 4.13 and 4.14 respectively. As noted previously, the passage vortex has vorticity of negative sign, while the counter and corner vortex have positive vorticity. Comparing the vorticity plots for 

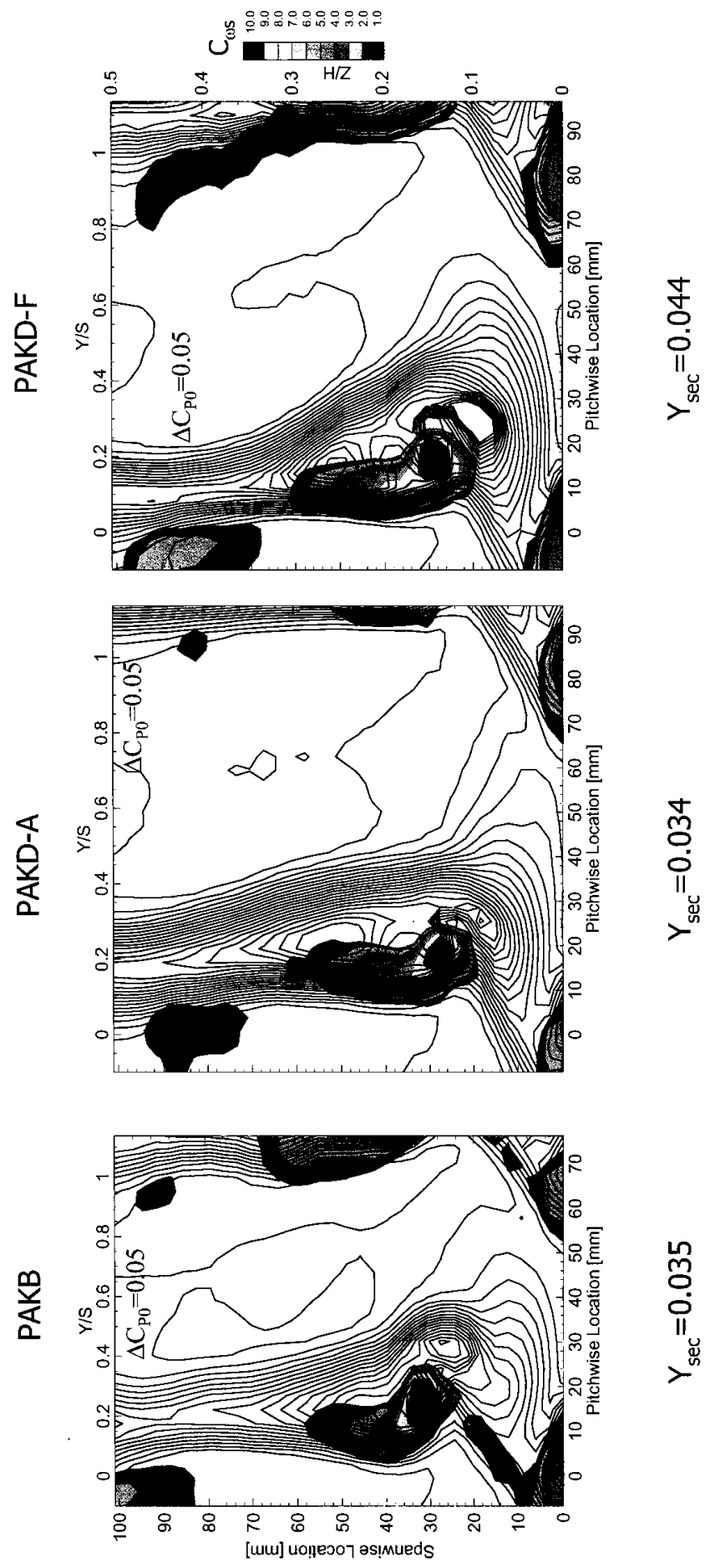

Figure 4.13: Positive Vorticity - Comparison of the Three Airfoils at FSTI=1.5\% 

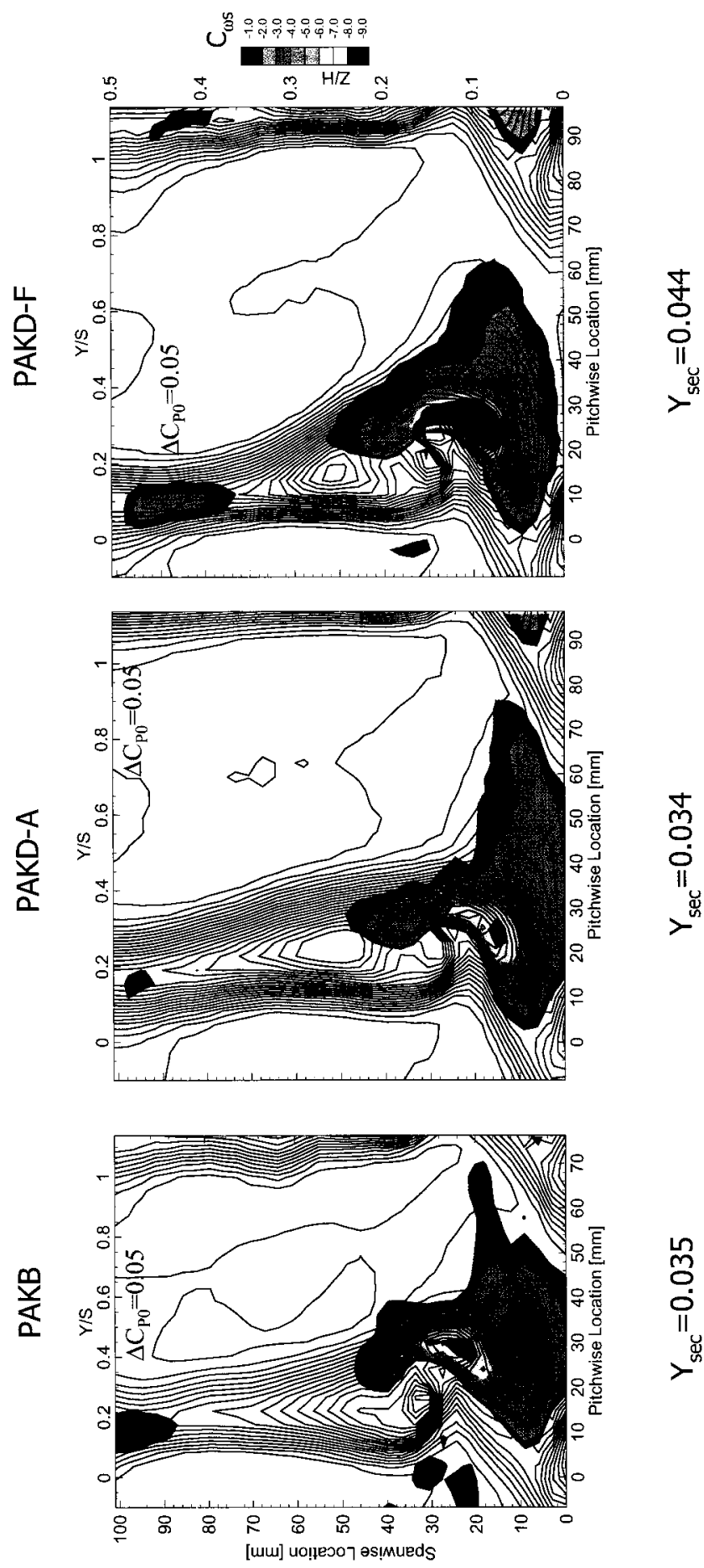

Figure 4.14: Negative Vorticity - Comparison of the Three Airfoils at FSTI=1.5\% 
Table 4.5: Comparison of Secondary Losses and Secondary Circulation for the Three Airfoils

\begin{tabular}{|l|c|c|c|}
\cline { 2 - 4 } \multicolumn{1}{c|}{} & PAKB & PAKD-A & PAKD-F \\
\hline$Y_{\text {TOTAL }}$ & 0.070 & 0.094 & 0.080 \\
\hline$Y_{\text {PROFILE }}$ & 0.035 & 0.060 & 0.036 \\
\hline$Y_{\text {SEC }}$ & 0.035 & 0.034 & 0.044 \\
\hline $\mathbf{C}_{\Gamma+}$ & 0.328 & 0.385 & 0.405 \\
\hline $\mathbf{C}_{\Gamma-}$ & -0.260 & -0.253 & -0.302 \\
\hline $\mathbf{C}_{\text {SKE }}{ }^{n}$ & 0.020 & 0.018 & 0.026 \\
\hline $\mathbf{Q}$ & 4.4 & 5.9 & 6.6 \\
\hline
\end{tabular}

PAKB and PAKD-A, it can be seen that the positive vorticity for PAKD-A extends further towards the midspan resulting in a greater value of positive circulation. The situation is opposite when it comes to the negative vorticity, where the strength of the passage vortex is slightly stronger for PAKB. It is believed that the presence of the pressure side separation bubble on PAKB may have contributed to the stronger negative circulation. Section 4.2 briefly discussed the influence of the pressure surface bubble on the secondary flows. Brear et al. (2002) investigated that phenomenon and concluded that the existence of the pressure side bubble may affect the secondary flows and losses such that it can strengthen the endwall flows and increase the magnitude of the losses. As mentioned earlier, PAKB has a bubble on the pressure side of the airfoil, while the PAKD-A has not. The author is of the opinion that this may be one of the factors in higher losses for the PAKB airfoil. The presence of the suction surface bubble also has an effect on the secondary losses. As mentioned previously in Section 4.2, Duden and Fottner (1999) observed an increase in secondary losses as a result of the suction surface separation bubble. Both, PAKB and PAKD-A, as shown in Figure 4.1, have suction surface bubbles. The effect of suction surface bubble on 
secondary flows cannot be quantified based on the present measurements. However, it is believed that the combined effect of the pressure and suction side separation bubbles on the PAKB losses is greater than that of suction side separation bubble of PAKD-A.

As can be seen from both the integrated results as well as vorticity plots, the PAKD-F has the highest magnitudes of losses, secondary circulation and massaveraged secondary kinetic energy. Figures 4.13 and 4.14 show that both the peak magnitudes as well as the extent of the vortical regions are the largest for PAKD-F. These results are in line with previous studies by Weiss and Fottner (1995), Benner et al. (2004a), Dossena et al. (2004), Hodson and Dominy (1987a), and Perdichizzi and Dossena (1993). All of the above investigators have been studying the effect of loading and/or loading distribution on the strength and magnitude of the secondary losses and have concluded that more highly loaded, and especially front-loaded airfoils tend to generate stronger secondary flows and hence, higher losses.

\subsubsection{Axial Velocity Distribution (AVR)}

The AVR contours for the three airfoils are shown in Figure 4.15. AVR was discussed into greater detail in Section 4.4.2. It was author's belief that the AVR overshoot is due to the relatively loss free fluid funnelled in a constricted stream tube between the two counter-rotating vortices, the corner vortex and the blade trailing edge.

Similar overshoots were also found in PAKD-A and PAKD-F measurements, with PAKD-F having the greatest magnitude and largest area involved with the overspeed. Although not known at present, it is possible that the strength of the overspeed may 
be related to the strength of secondary flows.

\subsubsection{The Effect of Varying Freestream Turbulence Intensity}

This section will discuss the influence of FSTI on the secondary losses. Figure 4.16 shows the total pressure coefficients for the three airfoils with FSTI=4.0\%. When compared to the same plot for FSTI of 1.5\% shown in Figure 4.12 (Section 4.5.1), it can be seen that the magnitudes of the mixed-out mass-averaged total pressure losses are lower at the higher FSTI level. In addition, the secondary flows are confined to the area of passage closer to the blade suction surface, and their pitchwise extent is reduced. The secondary flows are also displaced further away from the endwalls for all three blades. Another feature seen in Figure 4.16 is the apparent existence of only two distinct high loss peaks for the PAKD blades, as opposed to the three peaks visible from Figure 4.12. Higher FSTI promotes transition of the boundary layer from laminar to turbulent flow. If there is indeed a local two-dimensional boundary layer separation on suction side of the PAKD airfoils at about $25 \%$ of the span at lower FSTI, as suggested in Section 4.5.1, it would be expected that higher FSTI will promote earlier transition and flow reattachment, thus eliminating the third loss peak. However, these conclusions should be supported with further tests.

Figures 4.17 and 4.18 show the streamwise vorticity contours for all blades at FSTI $=4.0 \%$. The magnitude of the positive vorticity for all blades is reduced, while its spanwise extent is greater for FSTI of $4.0 \%$. However, integrated values of positive vorticity are roughly the same for both FSTI levels, as can be seen from Table 4.6. The negative vorticity (which is associated with the passage vortex) on the other hand shows both a decrease in its magnitude as well as in its spanwise extent. When compared to Figure 4.14 in Section 4.5.1, it can be seen that the negative vorticity 

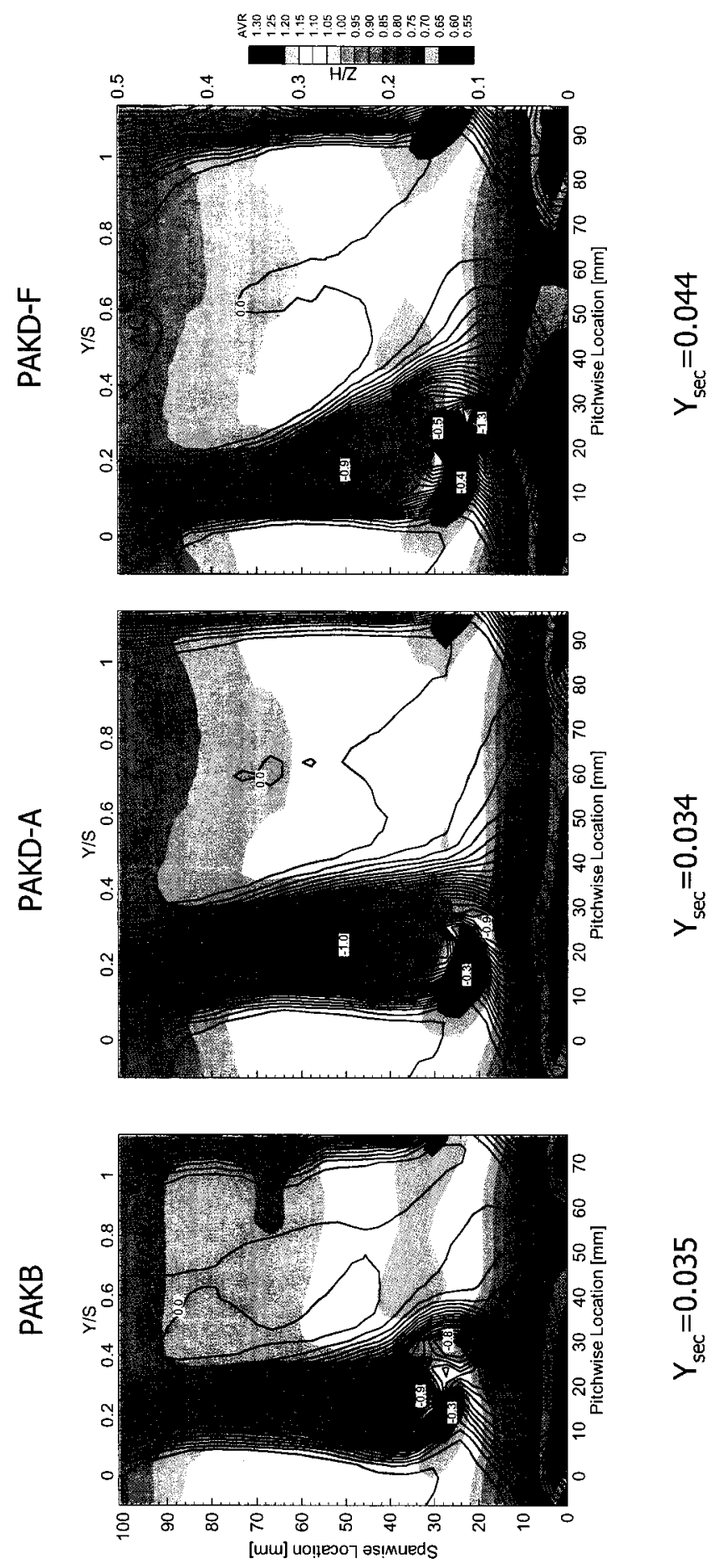

Figure 4.15: AVR Contours - Comparison of the Three Airfoils at FSTI=1.5\% 
is pushed towards the endwall, and that it extends further in the pitchwise direction. Greater decrease in the negative vorticity magnitude is observed for the PAKB and PAKD-A airfoils than for PAKD-F. This is manifested in a noticeable decrease in negative circulation for PAKB and PAKD-A, as seen in Table 4.6.

Adjlout and Dixon (1992) investigated the effect of higher FSTI level on the secondary losses and found that the total pressure loss coefficients and hence the secondary loss coefficients do not vary much with FSTI. They did not provide the loss magnitudes, but the pitchwise mass-averaged total pressure coefficient distribution at the plane farthest from the blade trailing edge did show somewhat lower losses in the endwall vicinity for cases with higher FSTI. The integrated secondary losses displayed in Table 4.6 show a reduction with increased FSTI levels. The reduction in secondary loss coefficient at FSTI of $4.0 \%$ is greater for PAKB and PAKD-A than for PAKD-F. In addition, the secondary circulation for the PAKD-F airfoil is almost unaffected by the change in FSTI. It is believed that the disappearance of the third loss peak is the major reason for the reduction in losses for PAKD-F. On the other hand, both PAKB and PAKD-A experience about a $20 \%$ reduction in the magnitude of the negative circulation, which shows that the secondary flows are indeed weaker at the higher turbulence level. Similar trends can be observed in the case of mass-averaged secondary kinetic energy magnitudes where decrease is greater for the PAKB and PAKD-A blades than for the PAKD-F. As was the case in measurements at lower FSTI, PAKB and PAKD-A again show comparable secondary loss magnitudes. The results are again counter intuitive as quality factor and total pressure coefficient contours indicate higher losses for the PAKD-A airfoil. However, higher profile losses and lower axial velocity in regions of high total pressure loss for the PAKD-A airfoil contribute to lower magnitude of secondary losses. 


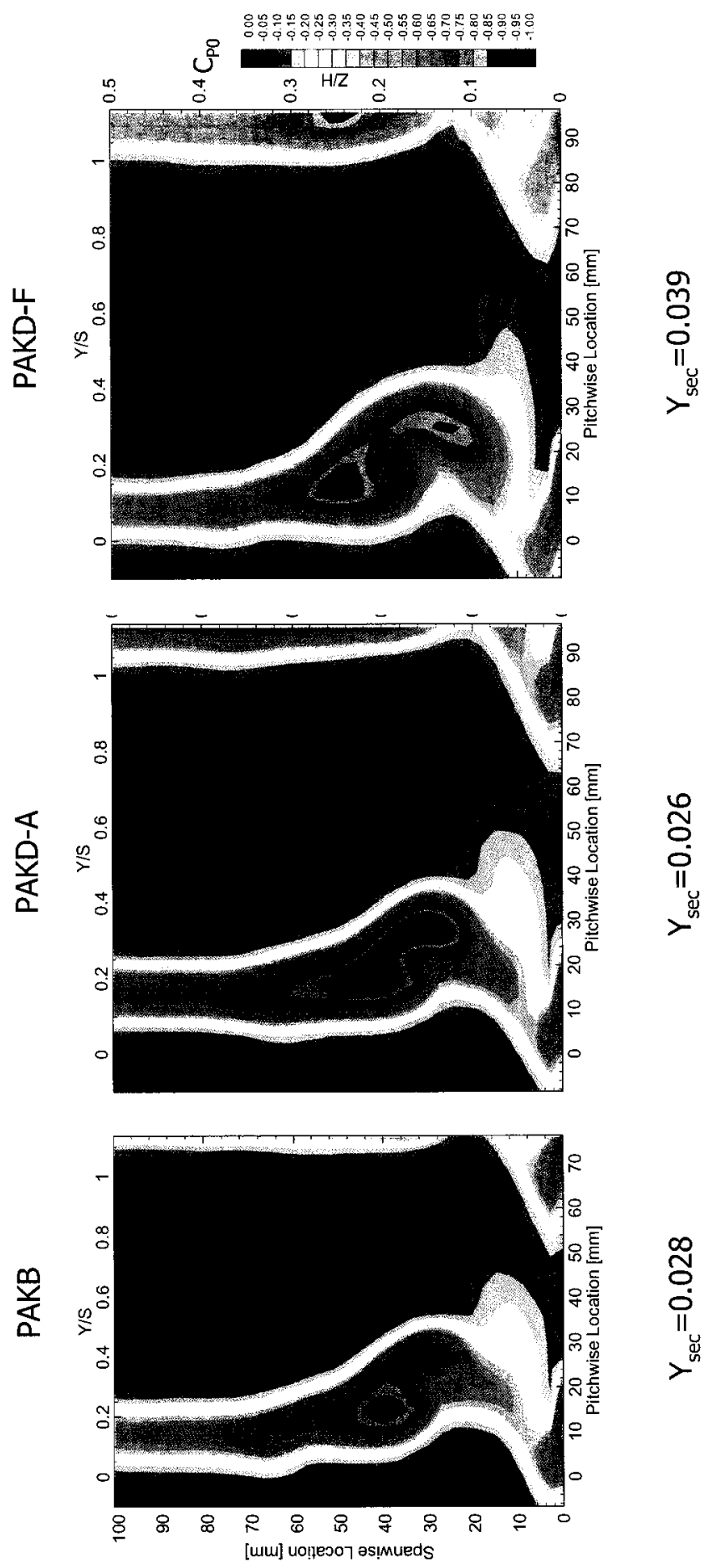

Figure 4.16: Total Pressure Loss Contours - Comparison of the Three Airfoils at $\mathrm{FSTI}=4.0 \%$ 

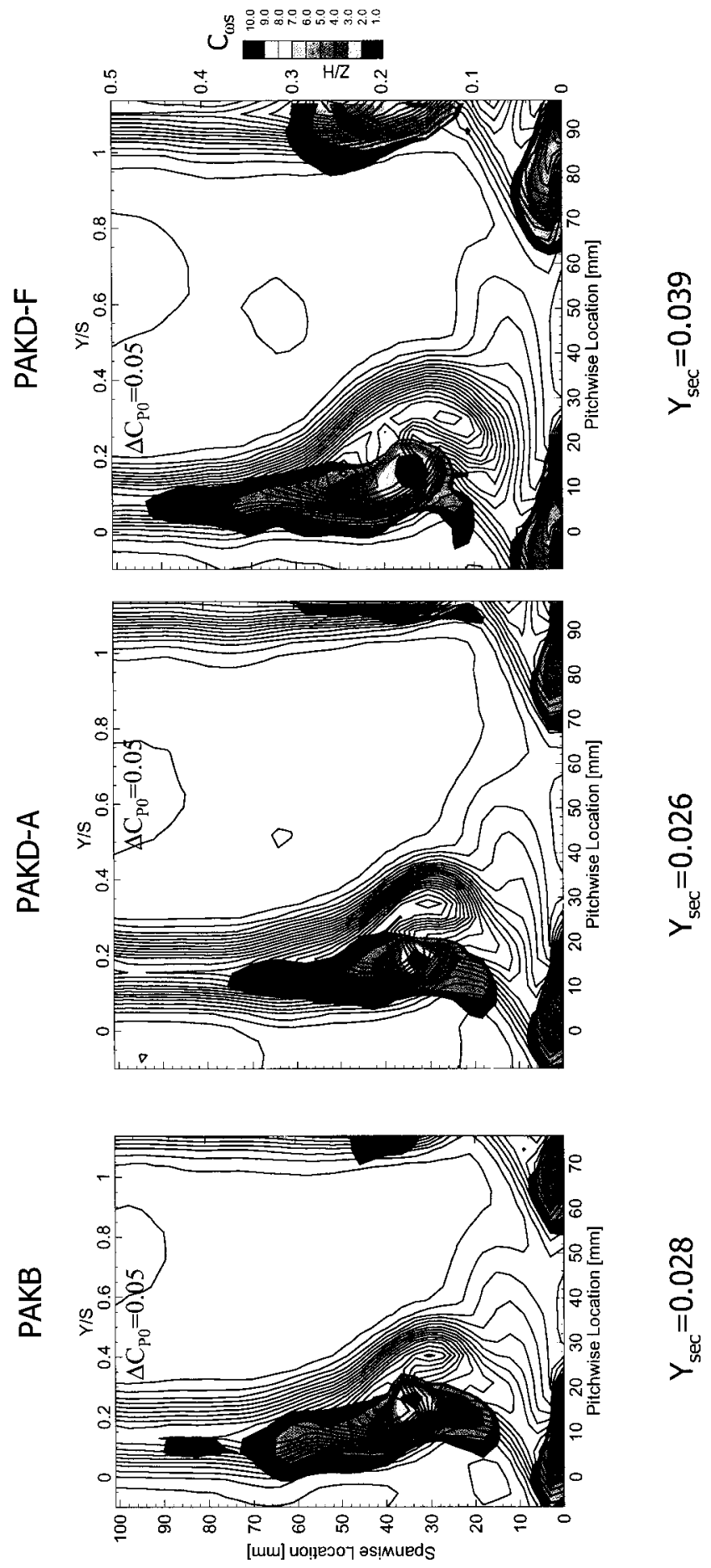

Figure 4.17: Positive Vorticity - Comparison of the Three Airfoils at FSTI=4.0\% 


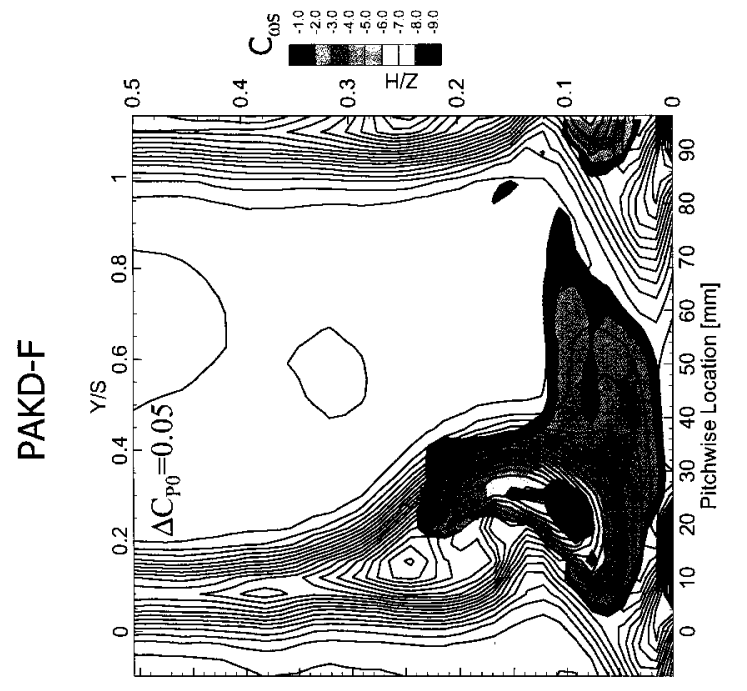

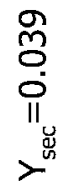

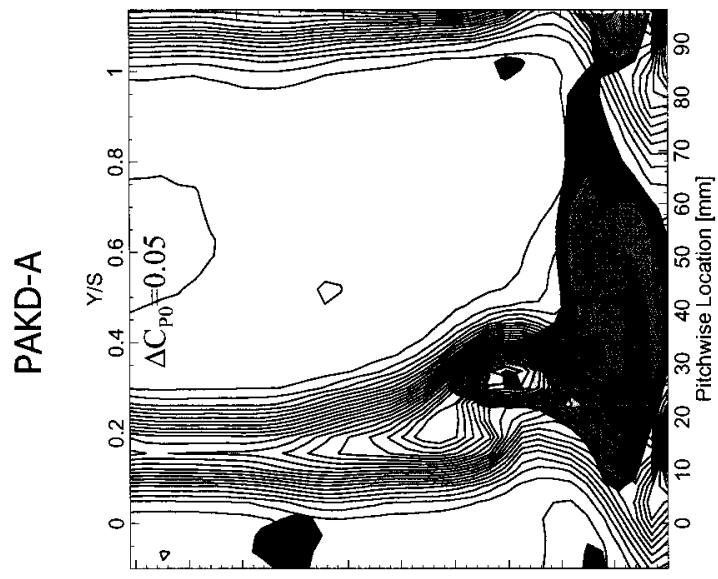

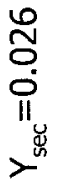

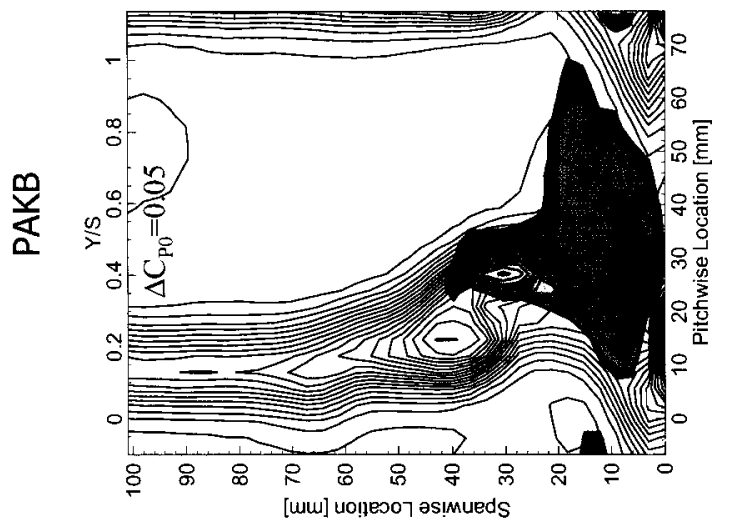

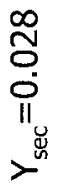

Figure 4.18: Negative Vorticity - Comparison of the Three Airfoils at FSTI=4.0\% 
Table 4.6: Comparison of Secondary Losses and Secondary Circulation for the Three Airfoils at FSTI of $1.5 \%$ and $4.0 \%$

\begin{tabular}{|l|c|c|c|c|c|c|}
\cline { 2 - 7 } \multicolumn{1}{c|}{} & \multicolumn{3}{c|}{ FSTI $=1.5 \%$} & \multicolumn{3}{c|}{ FSTI $=4.0 \%$} \\
\cline { 2 - 7 } & PAKB & PAKD-A & PAKD-F & PAKB & PAKD-A & PAKD-F \\
\hline$Y_{\text {TOTAL }}$ & 0.070 & 0.094 & 0.080 & 0.064 & 0.068 & 0.071 \\
\hline$Y_{\text {PROFILE }}$ & 0.035 & 0.060 & 0.036 & 0.036 & 0.042 & 0.032 \\
\hline$Y_{\text {SEC }}$ & 0.035 & 0.034 & 0.044 & 0.028 & 0.026 & 0.039 \\
\hline$C_{\Gamma+}$ & 0.328 & 0.385 & 0.405 & 0.338 & 0.377 & 0.406 \\
\hline$C_{\Gamma-}$ & -0.260 & -0.253 & -0.302 & -0.198 & -0.206 & -0.294 \\
\hline$C_{\text {SKE }}$ & 0.020 & 0.018 & 0.026 & 0.015 & 0.014 & 0.024 \\
\hline
\end{tabular}

As mentioned previously, Gregory-Smith and Cleak (1992) showed a reduction in net secondary losses with higher FSTI, but they were also of the opinion that high inlet turbulence alone has little effect on the secondary loss. They believed that the reduction in mixed-out losses was due to a thinned inlet boundary layer. What was also interesting in Gregory-Smith and Cleak's work was that at lower FSTI, a laminar separation bubble was present on the blade suction surface, while at FSTI of $5 \%$ there was no bubble. It is possible that the disappearance of the separation bubble at higher FSTI might have had an influence on the reduction in losses. Both PAKB and PAKD-A have separation bubbles while PAKD-F has not. It is widely accepted that separation bubbles are reduced in size with an increase in FSTI. This can be also verified through the loadings presented in Section 4.2. It is thus believed that the smaller separation bubbles have less of an effect on the secondary flows and losses, which is consistent with the results presented in Table 4.6. However, the need for more testing and investigating the effects of the presence of separation bubbles on suction and pressure sides of the airfoils and their influence on the secondary flows must be stressed again. 
Similar to what was seen in Section 4.5.2 where the results for FSTI of $1.5 \%$ were discussed, an axial velocity ratio (AVR) greater than 1.0 was observed again at the higher FSTI level. As can be seen in Figure 4.19, the peak magnitude and the area of high AVR has decreased noticeably, with PAKD-F again exhibiting the highest values of AVR. This supports the argument that the strength of the secondary flows and the AVR overshoot are related. The position of the areas experiencing high AVR are very similar to those already seen in Figure 4.15: that is, the areas showing higher AVRs are found in the relatively loss free fluid located between the two counterrotating vortices, the corner vortex, and the projection of the blade trailing edge. As mentioned, to gain more understanding about this phenomenon, more testing is required preferably at different traverse planes located closer to the trailing edge and perhaps within the blade passage.

\subsection{Summary of Results and Conclusions}

This chapter has presented and discussed the results obtained from the secondary flow measurements for PAKB, PAKD-A, and PAKD-F at design incidence. All measurements were conducted at a constant Reynolds number of 80,000 and two freestream turbulence intensities (1.5\% and $4.0 \%)$.

The integrated results have shown that the PAKB and PAKD-A airfoils generate comparable secondary losses. This was not in line with expectations as the quality factor suggested that there would be higher losses for PAKD-A. It is believed that higher losses for the PAKB airfoil may be attributed to the existence of the pressure and suction surface separation bubbles whose combined effect on the secondary losses is greater than that of the suction side separation bubble on the PAKD-A airfoil. The 


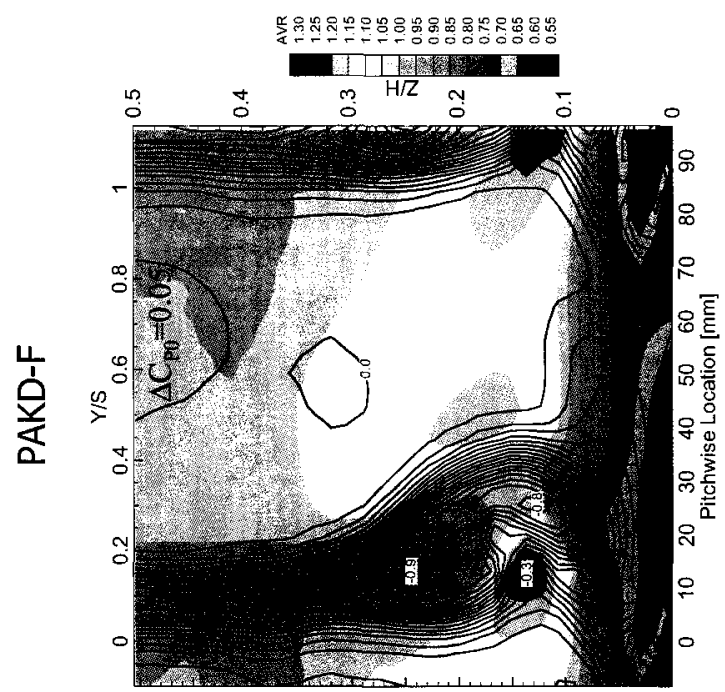

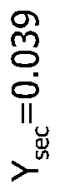
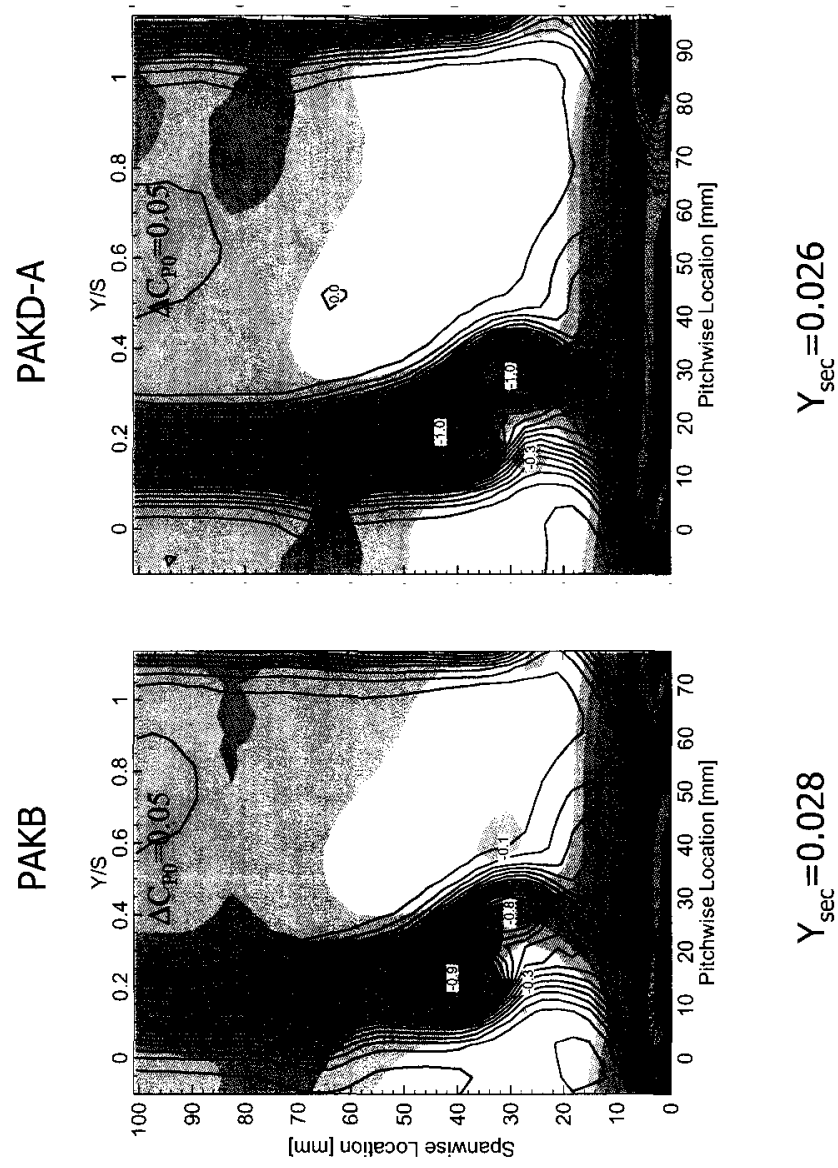

Figure 4.19: AVR Contours - Comparison of the Three Airfoils at FSTI $=4.0 \%$ 
highest secondary loss was generated by the PAKD-F airfoil.

An interesting feature was noticed in axial velocity ratio (AVR) contours. The AVR overshoots the mean value by as much as $30 \%$ in an area nested between the two counter-rotating vortices (the passage and counter vortex), the corner vortex and the projection of the blade trailing edge. The area of higher axial velocity was a region of relatively low total pressure loss. The highest overshoot was seen for PAKD-F airfoil. A relationship between the strength of the secondary flow and the AVR overshoot was suggested.

When the FSTI level was raised from $1.5 \%$ to $4.0 \%$, the magnitudes of the total pressure losses decreased. One of the effects of higher FSTI was the apparent elimination of the third loss peak that was previously seen at lower FSTI level for the PAKD airfoils. The total pressure contours showed confinement of the secondary flows closer to suction surface, a decrease in the pitchwise extent of the secondary flows and their lift-off from the endwall. The magnitudes of secondary vorticity, secondary kinetic energy, and AVR overshoots were generally reduced with increase in turbulence levels.

It was suggested that more testing was required to provide better understanding of the parameters influencing the secondary flows, such as the variation in inlet boundary layer thickness and turbulence levels, and effect of suction and pressure side separation bubbles. Flow visualization was also recommended as a tool that would provide more insight into the flow physics.

This concludes the discussion of the results at design incidence. The next chapter will focus on the results obtained from measurements at off-design incidence. 


\section{Chapter 5}

\section{Secondary Flows at Off-Design Incidence}

\subsection{Introduction}

This chapter will present and discuss the results obtained from the secondary flow measurements at off-design incidence values of $-5^{\circ}$ and $+5^{\circ}$, and compare them to those obtained at the design incidence. The measurements were performed at a constant Reynolds number of 80,000 (based on axial chord and inlet velocity) and FSTI of $1.5 \%$ and $4.0 \%$. Table 5.1 summarizes the cases examined in the present work. No measurements were made at $-5^{\circ}$ incidence and FSTI of $4.0 \%$. This is because the closely-mounted grid used to generate $4.0 \%$ turbulence interferes with the test section floating wall shown in Figure 3.3, Section 3.4. The installation of the grid at $-5^{\circ}$ incidence would require major modifications to the test section. The analysis of the results at FSTI of $1.5 \%$ have shown that at negative incidence, the 
Table 5.1: Summary of the Secondary Loss Measurements

\begin{tabular}{|c|c|c|c|c|c|c|}
\cline { 2 - 7 } \multicolumn{1}{c|}{} & \multicolumn{2}{c|}{ PAKB } & \multicolumn{2}{c|}{ PAKD-A } & \multicolumn{2}{c|}{ PAKD-F } \\
\cline { 2 - 7 } \multicolumn{1}{c|}{} & $1.5 \%$ & $4.0 \%$ & $1.5 \%$ & $4.0 \%$ & $1.5 \%$ & $4.0 \%$ \\
\hline$-5 \mathrm{deg}$ & $\boldsymbol{x}$ & & $\boldsymbol{x}$ & & $\boldsymbol{x}$ & \\
\hline $0 \mathrm{deg}$ & $\boldsymbol{x}$ & $\boldsymbol{x}$ & $\boldsymbol{x}$ & $\boldsymbol{x}$ & $\boldsymbol{x}$ & $\boldsymbol{x}$ \\
\hline$+5 \mathrm{deg}$ & $\boldsymbol{x}$ & $\boldsymbol{x}$ & $\boldsymbol{x}$ & $\boldsymbol{x}$ & $\boldsymbol{x}$ & $\boldsymbol{x}$ \\
\hline
\end{tabular}

secondary losses decreased. Other investigators (e.g. Yamamoto and Nouse (1988), Perdichizzi and Dossena (1993), etc.) have seen a decrease in secondary losses with reduction in blade incidence. Based on this it was concluded that positive incidence is more important in terms of secondary loss generation, and the decision was made to omit testing at $-5^{\circ}$ incidence and FSTI of $4.0 \%$.

The present chapter will be organized in the following way: Section 5.2 will present the loadings for the three airfoils at design and off-design incidence. The following section will discuss the inlet endwall boundary layers. Section 5.4 will provide a detailed discussion of the secondary flows and the parameters affecting them for the PAKB airfoil. The subsequent section will compare the off-design results for all three airfoils. Finally, the last section will provide a summary of conclusions for measurements at off-design incidence.

\section{$5.2 \quad$ Blade Loadings}

As was seen in the previous chapter, the blade loading, its distribution and the appearance of the separation bubbles on either of the airfoil surfaces have a substantial effect on the secondary flows and losses. 
Figure 5.1 shows the midspan loadings for all three airfoils at FSTI of $1.5 \%$ at all three values of incidence examined. It can be seen that the loading increases with incidence, and vice versa. As will be shown in Sections 5.4.1 and 5.5, secondary losses follow the same trends. This is due to the increased loading on the front part of the airfoil, which, as was shown in the previous chapter, increases the strength of the secondary flows and results in higher secondary losses. The middle plot in Figure 5.1 shows the loading for PAKD-A. It can clearly be seen that at positive incidence the flow separates from the suction surface at about $0.50 C_{x}$ and the PAKD-A airfoil stalls. The stall, as will be seen in Section 5.5 has a significant effect on the secondary flows. PAKD-F, however, has a suction surface separation bubble at negative incidence only, as seen in Figure 5.1. Popovic (2005) argued that the reason for the existence of the separation bubble on the PAKD-F suction surface at $-5^{\circ}$ is due to the later transition from laminar to turbulent flow. Popovic (2005) also concluded that based on the loading measurements, it was not possible to confirm with certainty existence of the pressure side separation bubble at incidence of $-5^{\circ}$. As was already discussed in the previous chapter, which presented results obtained from measurements at the design incidence, separation bubbles affect secondary flows and may lead to increases in secondary losses.

Figure 5.2 shows the loadings for the three airfoils at FSTI of $4.0 \%$ and design incidence and +5 degrees of incidence. Comparing two FSTI levels it can be seen that the most significant difference between them is that the PAKD-A airfoil at positive incidence and FSTI of $4.0 \%$ no longer stalls. A separation bubble is present on the on the suction surface but it reattaches at $x / C_{x}$ of about 0.75 . 

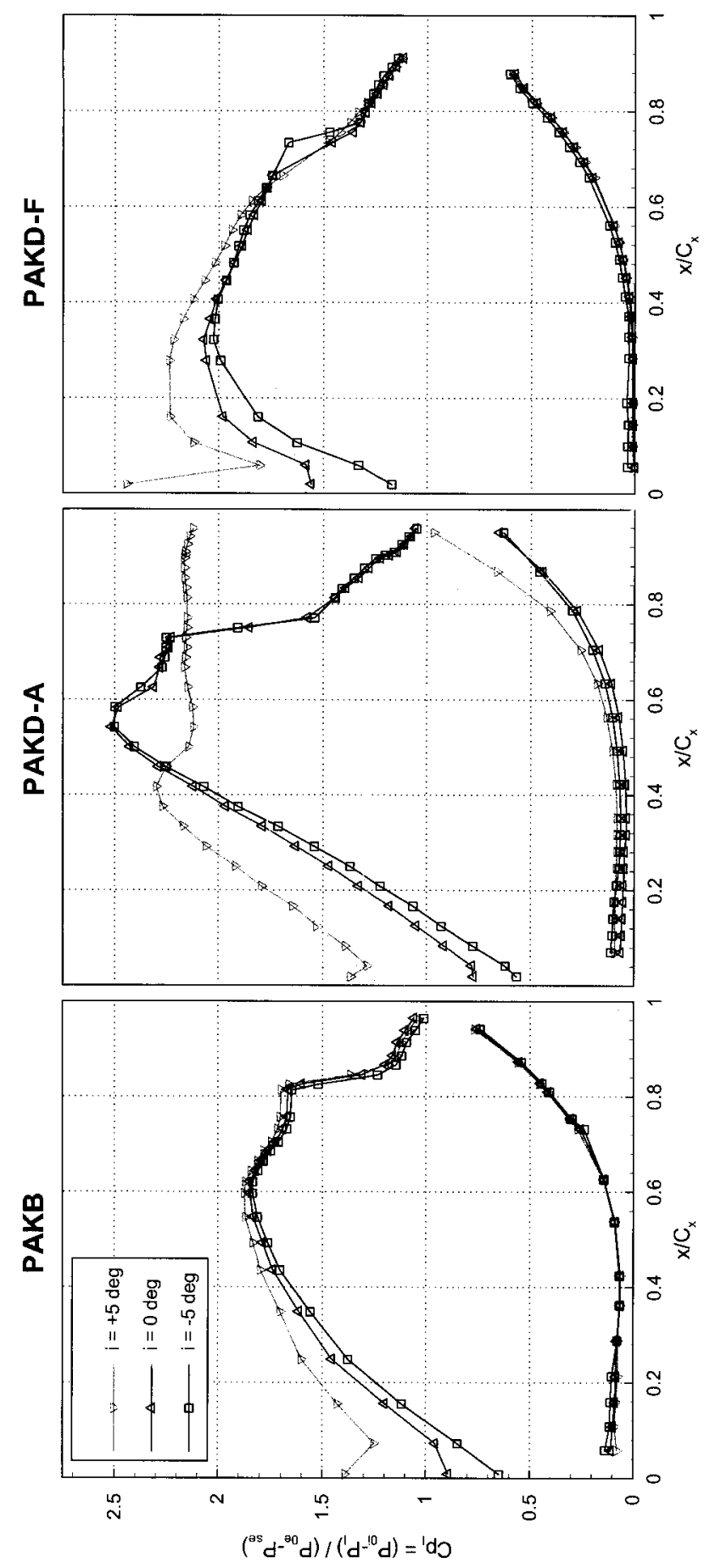

Figure 5.1: Blade Loadings at Off-Design Incidence at FSTI $=1.5 \%$ 


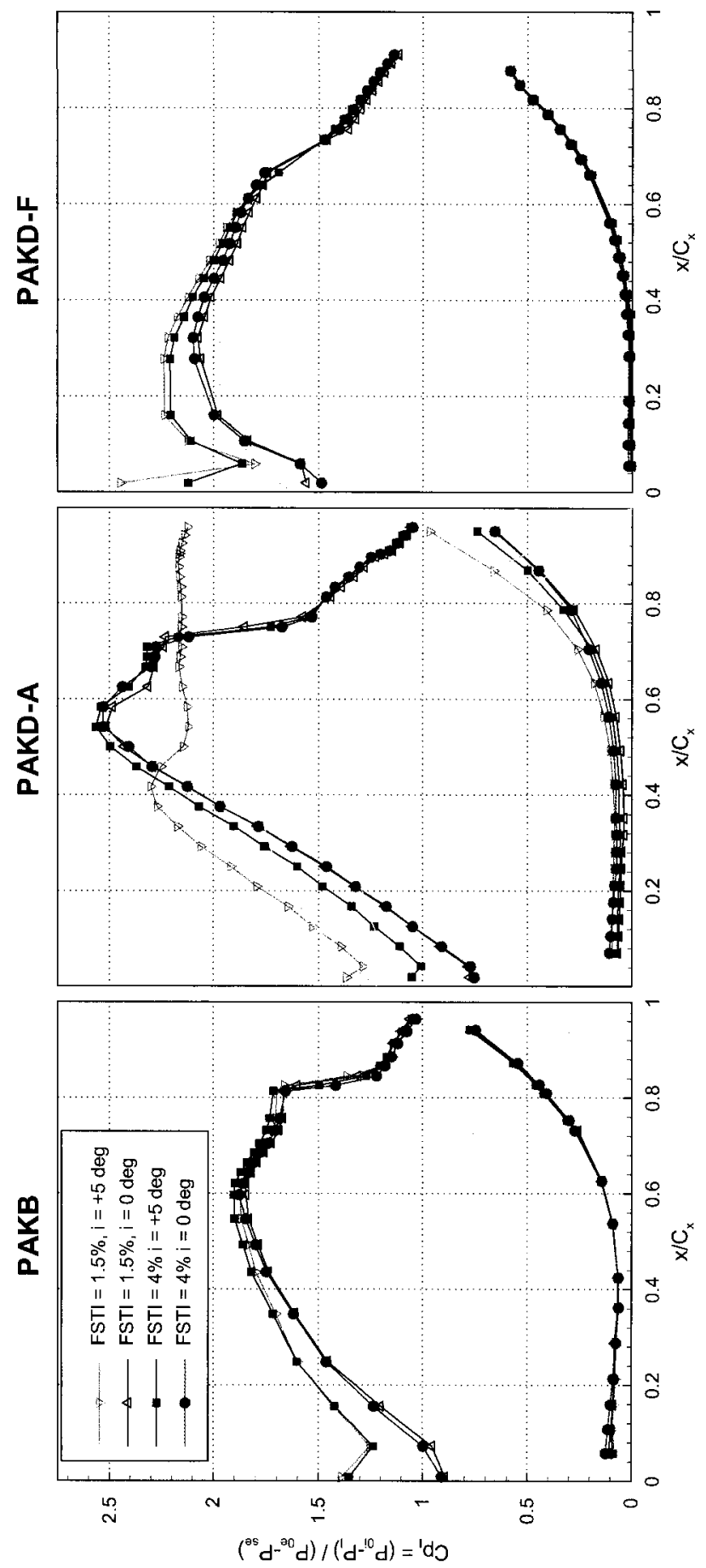

Figure 5.2: Blade Loadings at Off-Design Incidence at FSTI $=1.5 \%$ and $4 \%$ 


\subsection{Inlet Endwall Boundary Layers}

The non-uniformities arising from the turbulence generating grids were discussed in more detail in the previous chapter. Testing at off-design incidence involves the rotation of the turntable, as discussed in Section 3.2. As the turntable is rotated, the streamtube that enters the passage of interest changes. Thus, to account for the nonuniformities present in the inlet flow at off-design incidence, upstream measurements were made in the same fashion as for the design incidence: that is, a full rectangle covering 1.2 blade pitches and half span was traversed at $1.2 C_{x}$ upstream of the middle blade leading edge.

Tables 5.2 and 5.3 summarize the resulting inlet total pressure coefficients and boundary layer parameters, respectively.

Table 5.2: Summary of Integrated Inlet Total Pressure Coefficients at Design and Off-Design Incidence

\begin{tabular}{|c|c|c|c|c|c|}
\hline & \\
\hline & & & $-5 \mathrm{deg}$ & Odeg & $+5 \mathrm{deg}$ \\
\hline \multirow{6}{*}{$\mathrm{C}_{\mathrm{PO}}{ }^{\prime \prime}$} & \multirow{3}{*}{$1.5 \%$} & PAKB & -0.008 & -0.017 & -0.011 \\
\hline & & PAKD-A & -0.009 & -0.012 & -0.014 \\
\hline & & PAKD-F & -0.009 & -0.012 & -0.012 \\
\hline & \multirow{3}{*}{$4.0 \%$} & PAKB & - & -0.036 & -0.029 \\
\hline & & PAKD-A & - & -0.035 & -0.031 \\
\hline & & PAKD-F & - & -0.035 & -0.030 \\
\hline
\end{tabular}

As mentioned in the previous chapter, the $\delta / h$ shown in Table 5.3 represents the obtained boundary layer thickness non-dimensionalized by the blade height (span). $\delta^{*}$ is boundary layer displacement thickness and $\theta$ is the momentum thickness. $H_{S F}$ is the shape factor, defined as ratio $\delta^{*} / \theta$. 
Table 5.3: Summary of Inlet Boundary Layer Parameters at Design and OffDesign Incidence

\begin{tabular}{|c|c|c|c|c|}
\cline { 2 - 5 } \multicolumn{2}{c|}{} & $-5 \mathrm{deg}$ & 0 deg & $+5 \mathrm{deg}$ \\
\hline \multirow{4}{*}{$1.5 \%$} & $\delta / \mathrm{h}$ & 0.17 & 0.18 & 0.16 \\
\cline { 2 - 5 } & $\delta^{*}[\mathrm{~mm}]$ & 4.1 & 4.0 & 3.9 \\
\cline { 2 - 5 } & $\theta[\mathrm{mm}]$ & 2.9 & 2.9 & 2.8 \\
\cline { 2 - 5 } & $\mathbf{H}_{\mathrm{SF}}$ & 1.4 & 1.4 & 1.4 \\
\hline \multirow{4}{*}{$4.0 \%$} & $\delta / \mathrm{h}$ & & 0.10 & 0.10 \\
\cline { 2 - 5 } & $\delta^{*}[\mathrm{~mm}]$ & & 1.4 & 0.8 \\
\cline { 2 - 5 } & $\theta[\mathrm{mm}]$ & & 1.1 & 0.6 \\
\cline { 2 - 5 } & $H_{\mathrm{SF}}$ & & 1.3 & 1.3 \\
\cline { 4 - 5 } & & &
\end{tabular}

\subsection{Discussion of the Results for the Baseline Cas- cade}

\subsubsection{Total Pressure and Vorticity Distributions}

This section will focus on the results obtained from the secondary flow measurements on PAKB at an off-design incidence of $-5^{\circ}$ and $+5^{\circ}$. The next section will compare the results for all three airfoils at off-design conditions.

The effect of positive and negative off-design incidence on the secondary flows has been investigated by a number of researchers, such as Yamamoto and Nouse (1988), Perdichizzi and Dossena (1993), Hodson and Dominy (1987b), and more recently Dossena et al. (2004). Benner et al. (2004b) focused their investigation on the effect of positive incidence only. All investigators reported an increase in secondary losses at positive incidence. Similarly, a decrease in losses was observed at negative incidence. The total pressure coefficient, positive and negative vorticity contours for the PAKB airfoil at all values of incidences examined are shown in Figures 5.3, 5.4, and 5.5, 
respectively. As in the previous chapter, the darker blue color indicates higher total pressure losses, whereas red regions indicate lower losses. Figure 5.3 shows that the secondary flows in the PAKB passage are forced away from the endwall and expanded in the spanwise direction with increasing incidence. The opposite effect is observed when incidence is decreased. The mixed-out mass-averaged losses shown in Table 5.4 show that the mixed-out secondary losses increase with increasing incidence.

The positive streamwise vorticity contours seen in Figure 5.4 do not show significant changes in the pitch and spanwise extent of the flow with the positive vorticity as the incidence changes, but an increase/decrease in its magnitude is observed with increase/decrease in incidence. This can also be verified from Table 5.4 where the integrated positive circulation increases with incidence. The negative vorticity, which is associated with the passage vortex, shows that the passage vortex increases in size, both in the pitchwise and spanwise directions, as well as in its magnitude with increasing incidence. The opposite trend is observed when the incidence is reduced. The integrated values of negative circulation shown in Table 5.4 confirm the impression given by the contour plot. Similar conclusions can also be drawn from the integrated secondary kinetic energy (SKE) presented in the table. An increase in SKE is evident with incidence.

Table 5.4: PAKB: Secondary Losses and Circulation Variation with Incidence

\begin{tabular}{|l|c|c|c|}
\cline { 2 - 4 } \multicolumn{1}{c|}{} & $-5 \mathrm{deg}$ & $0 \mathrm{deg}$ & $+5 \mathrm{deg}$ \\
\hline $\mathbf{Y}_{\mathrm{SEC}}$ & 0.026 & 0.035 & 0.041 \\
\hline $\mathrm{C}_{\Gamma+}$ & 0.260 & 0.328 & 0.375 \\
\hline $\mathrm{C}_{\Gamma-}$ & -0.195 & -0.260 & -0.307 \\
\hline $\mathbf{C}_{\mathrm{SKE}}{ }^{\prime \prime}$ & 0.014 & 0.020 & 0.027 \\
\hline
\end{tabular}




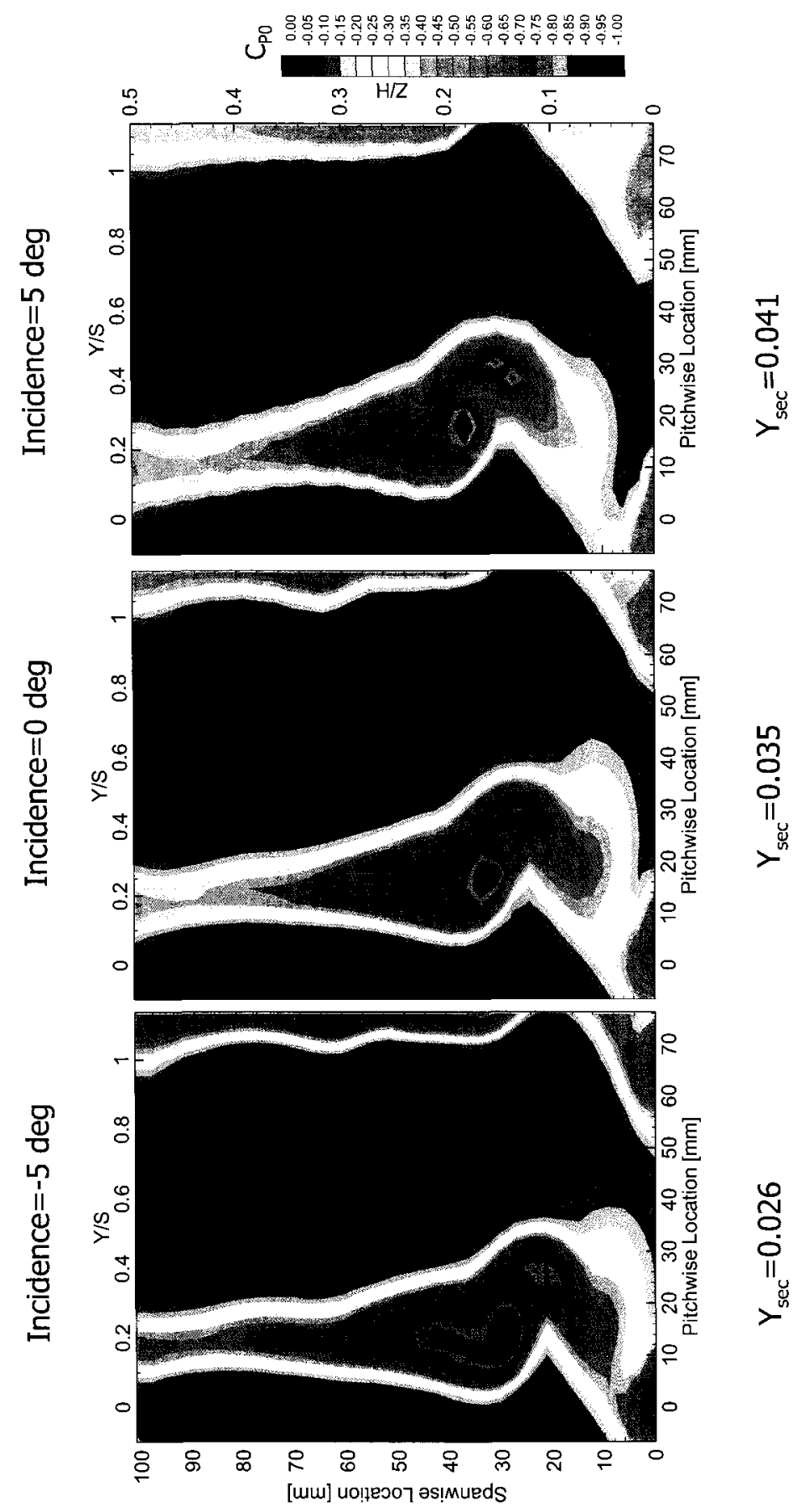

Figure 5.3: PAKB: Total Pressure Loss Contours at Design and Off-Design Incidence at $\mathrm{FSTI}=\mathbf{1 . 5 \%}$ 

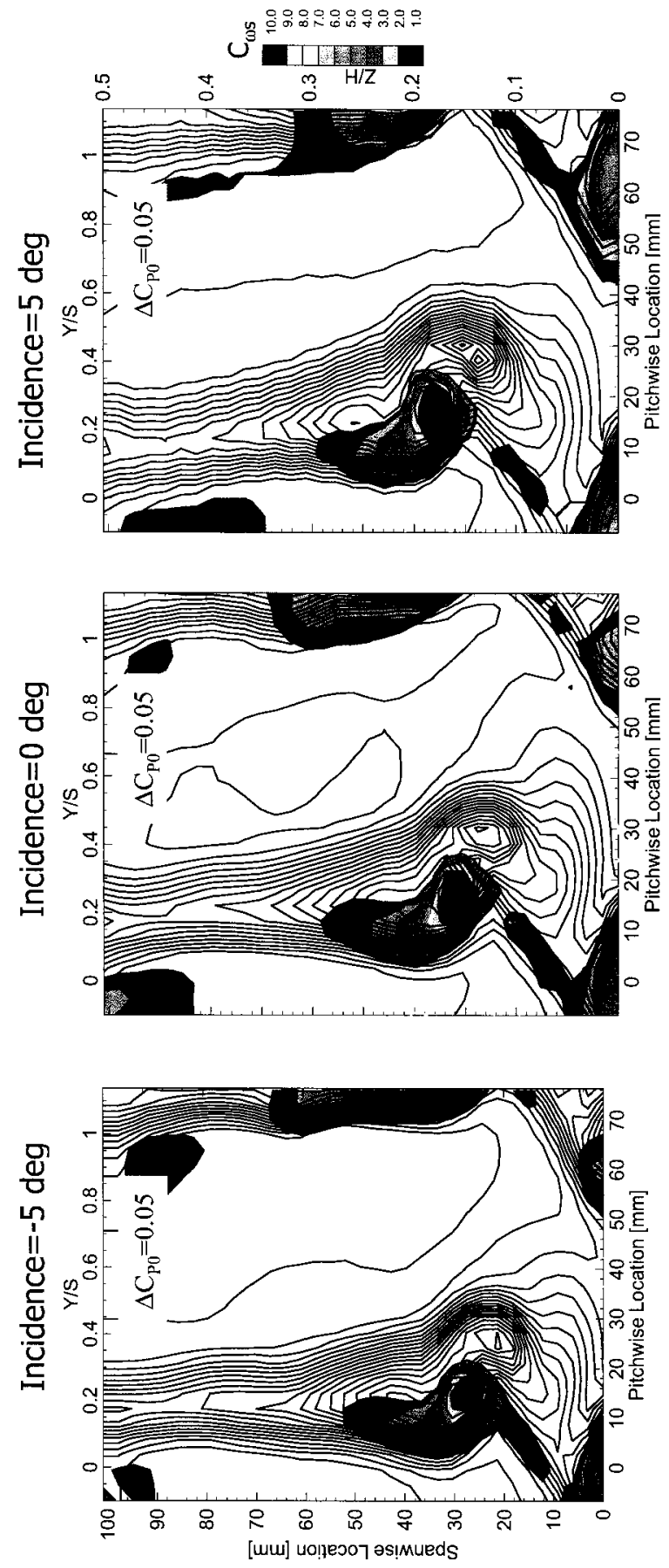

Figure 5.4: PAKB: Positive Vorticity at Design and Off-Design Incidence at FSTI $=1.5 \%$ 

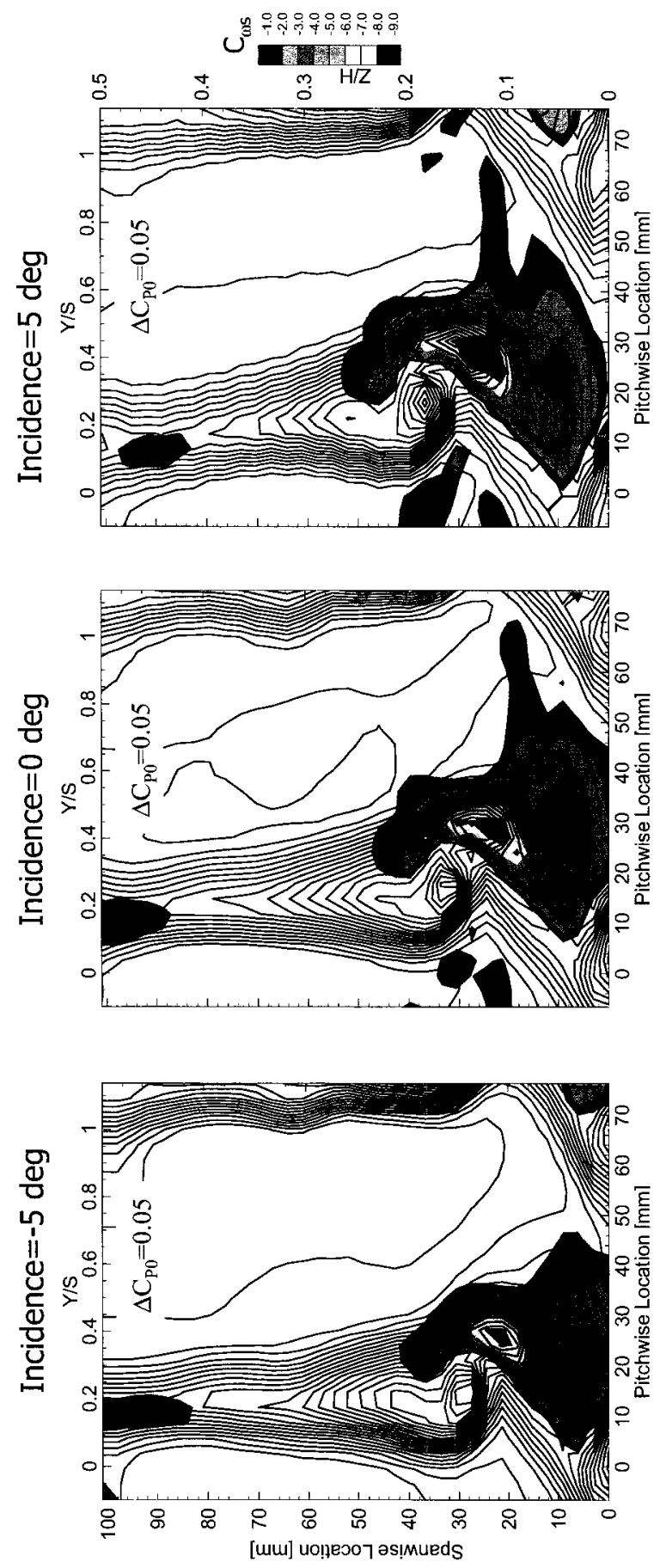

Figure 5.5: PAKB: Negative Vorticity at Design and Off-Design Incidence at FSTI $=1.5 \%$ 


\subsubsection{Axial Velocity Distributions (AVR)}

The AVR contours for design incidence were discussed in Chapter 4. A relationship between the secondary loss magnitude and the AVR overshoot was observed. Specifically, it was noticed that the AVR overshoots are higher for cases with stronger secondary flows. It is believed that because of the stronger secondary flows (stronger and larger vortices), the increased blockage forces the axial velocity up in the flow area nested between vortices thus increasing the AVR overshoot.

Figure 5.6 presents the AVR contours for PAKB at lower FSTI (1.5\%) and the three values of incidences examined, $-5^{\circ}, 0^{\circ}$, and $+5^{\circ}$. It can be seen that the AVR overshoot increases with increasing incidence, and that at $+5^{\circ}$ it occupies a larger area and has higher peaks than for the other two cases tested. Figures 5.4 and 5.5, presented in the previous section, showed an increase in the strength of the passage, counter and corner vortices with increasing incidence. There is clearly a relationship between the AVR overshoot and the strength of the secondary flows as the incidence varies. As was previously mentioned, increasing incidence increases the loading on the front part of the airfoil. The previous chapter has shown that front-loaded airfoils have stronger secondary flows and hence higher AVR overshoots than their aft-loaded counterparts. Therefore, an airfoil subjected to positive incidence produces higher AVR overshoots, suggesting again that the strength of the overshoot is associated indirectly with the increased loading on the front part of the airfoil.

\subsubsection{The Effect of Varying FSTI}

As discussed in the opening section of this chapter, no negative incidence measurements were conducted at FSTI of 4.0\%. Therefore, all comparisons regarding 

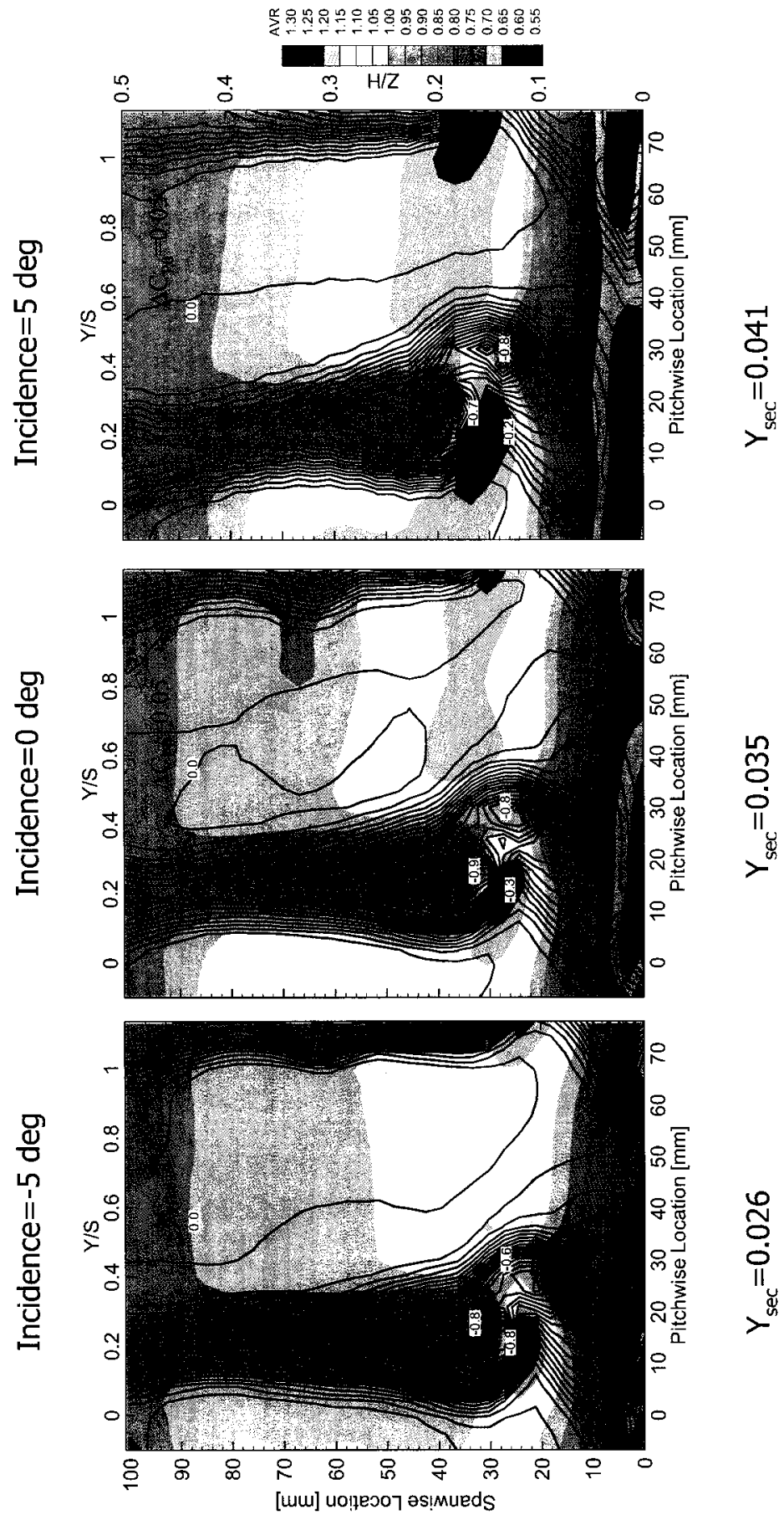

Figure 5.6: PAKB: AVR Distribution at Design and Off-Design Incidence at $\operatorname{FSTI}=1.5 \%$ 
the influence of FSTI on secondary flows at off-design incidence will be done for $+5^{\circ}$ only.

The effect of higher level of FSTI on the secondary flows and losses at design incidence was discussed in detail in the previous chapter. The major conclusion that was drawn was that higher FSTI reduces the magnitude of the secondary flows and the mass-averaged secondary losses. At the same time migration of the secondary flows away from the endwall and their confinement closer to the suction surface was observed at higher FSTI. The same general trend is observed when PAKB is subjected to $+5^{\circ}$ incidence.

Figure 5.7 shows the total pressure coefficient contours and the values of mixedout secondary loss coefficients for PAKB at the two FSTI levels. Again, as was the case at the design incidence, testing at off-design showed the same behavior: vorticity magnitudes and secondary losses decreased with increase in FSTI level, as can be seen in Figures 5.8 and 5.9. The values of integrated streamwise vorticity (secondary circulation), SKE and mixed-out mass-averaged secondary losses for the two FSTI levels at incidence of +5 are given in Table 5.5 .

Table 5.5: PAKB: Secondary Losses and Circulation Variation with FSTI at $+5^{\circ}$ Incidence

\begin{tabular}{|l|c|c|}
\cline { 2 - 3 } \multicolumn{1}{c|}{} & FSTI=1.5\% & FSTI=4.0\% \\
\hline $\mathbf{Y}_{\text {SEC }}$ & 0.041 & 0.037 \\
\hline $\mathbf{C}_{\Gamma+}$ & 0.375 & 0.346 \\
\hline $\mathbf{C}_{\Gamma-}$ & -0.307 & -0.234 \\
\hline $\mathbf{C}_{\text {SKE }}{ }^{\prime \prime}$ & 0.027 & 0.018 \\
\hline
\end{tabular}



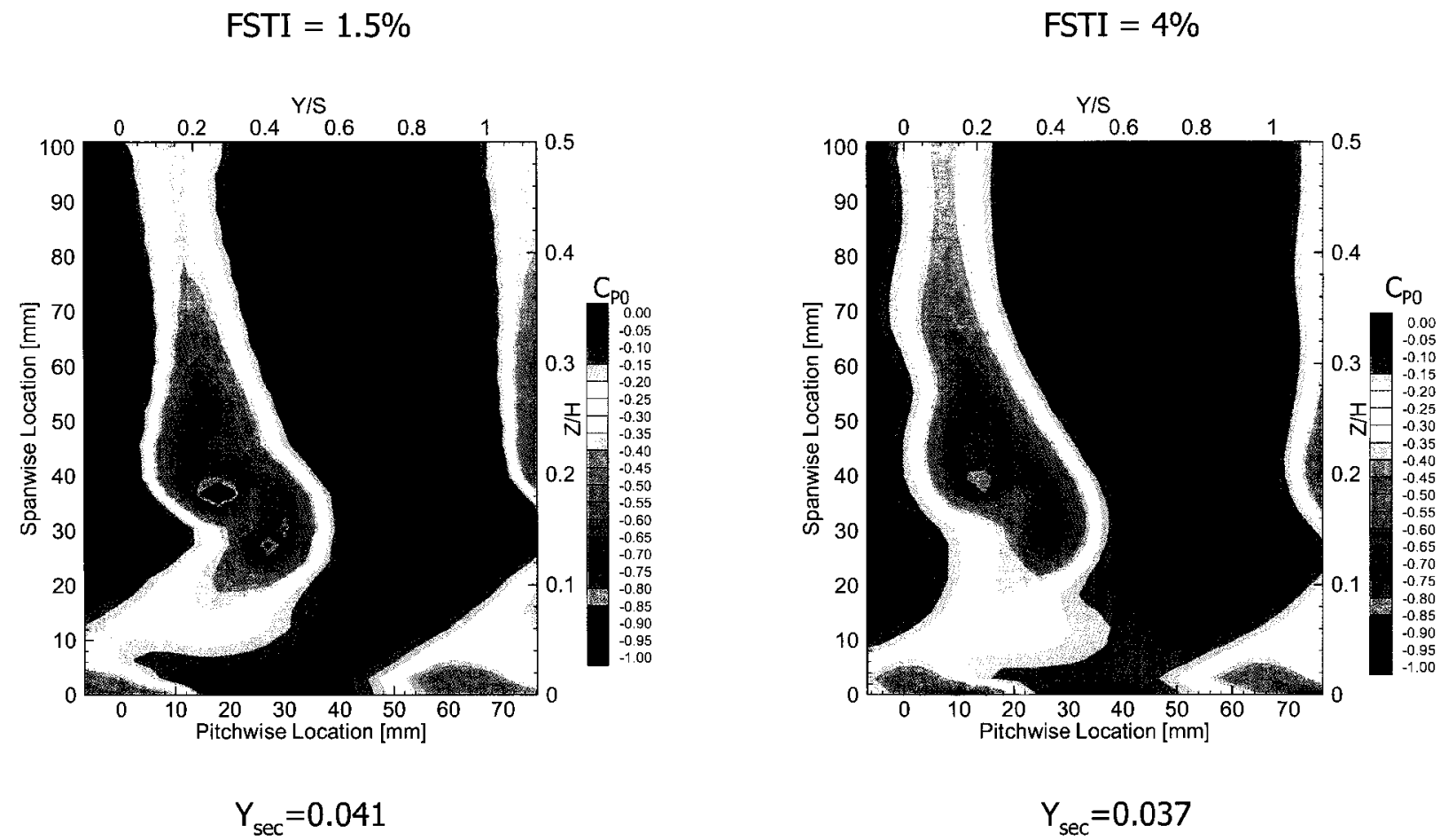

Figure 5.7: PAKB: Total Pressure Contours at $+5^{\circ}$ Incidence and FSTI of $1.5 \%$ and $4.0 \%$

\subsection{Comparison of Results for All Three Cascades}

\subsubsection{Total Pressure and Vorticity Distributions}

The previous section discussed the effect of changing incidence on secondary flows for the baseline airfoil, PAKB. The results have shown that the secondary losses increase with increasing incidence and vice versa. This was expected and is in line with previous investigations.

Mixed-out mass-averaged secondary loss coefficients for the three cascades and all investigated cases are provided in Figure 5.10. The secondary loss coefficient 

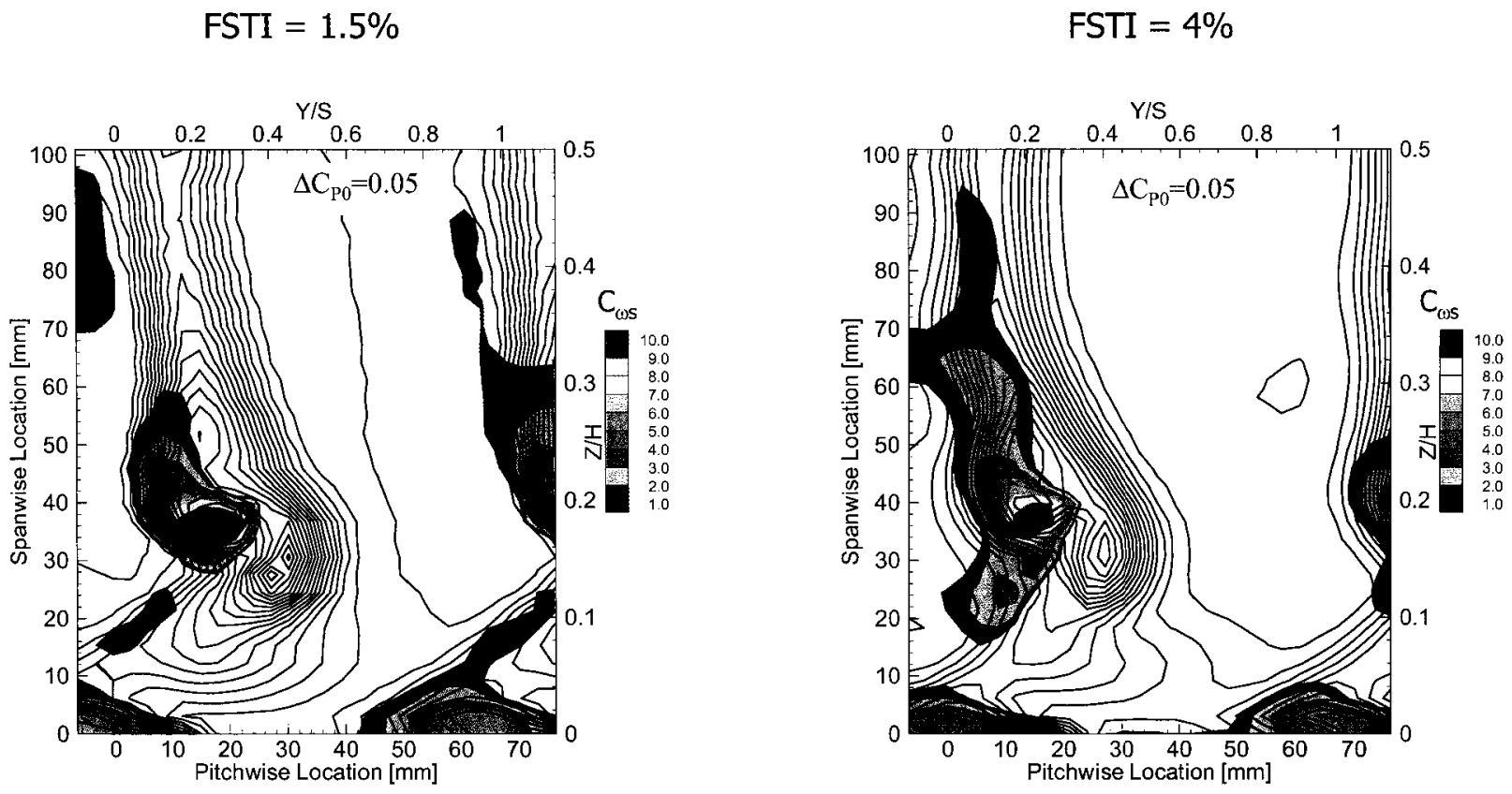

Figure 5.8: PAKB: Positive Vorticity at $+5^{\circ}$ Incidence and FSTI of $1.5 \%$ and $4.0 \%$

$$
\mathrm{FSTI}=1.5 \%
$$

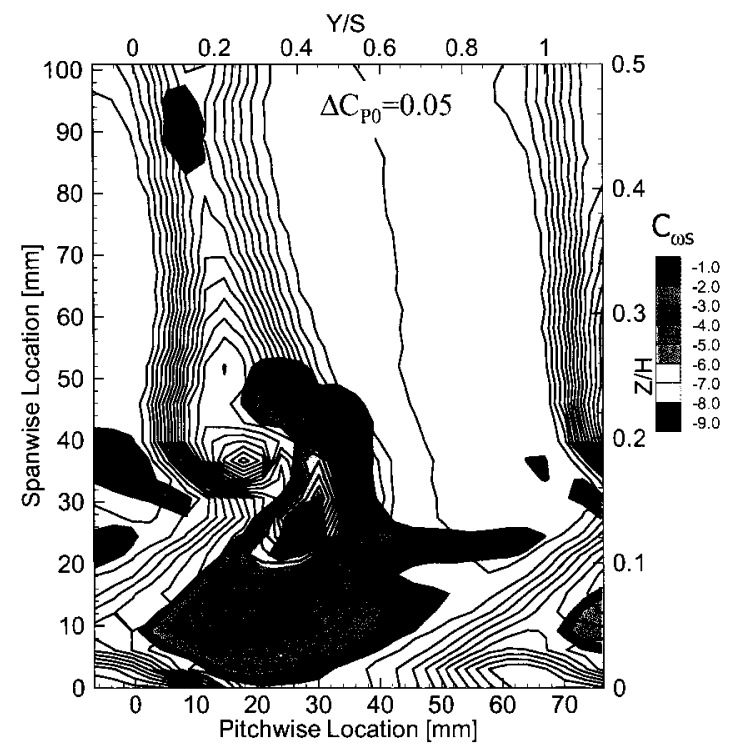

$$
\mathrm{FSTI}=4 \%
$$

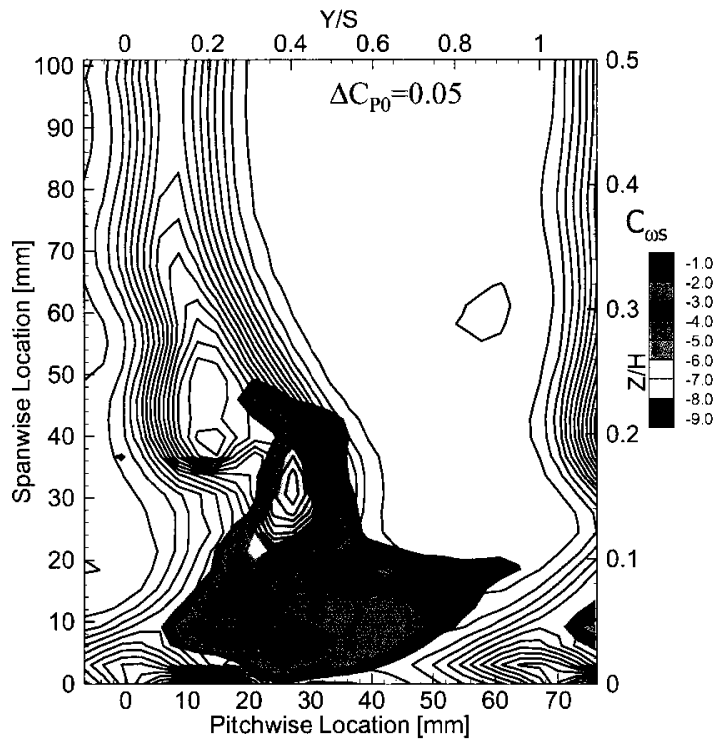

Figure 5.9: PAKB: Negative Vorticity at $+5^{\circ}$ Incidence and FSTI of $1.5 \%$ and $4.0 \%$ 
quantities are also summarized in Table 5.6, while the remaining experimental results for measurements at off-design incidence are given in Appendix E. It can be seen that the behavior of the PAKD-F airfoil is similar to that already seen for PAKB and in previous studies: secondary losses increase with positive incidence and vice versa. For all cases, the losses for PAKD-F are greater than those for PAKB. Similar to results at design incidence, PAKD-A and PAKB have comparable secondary losses for all but one case $\left(+5^{\circ}\right.$, FSTI of $\left.1.5 \%\right)$.

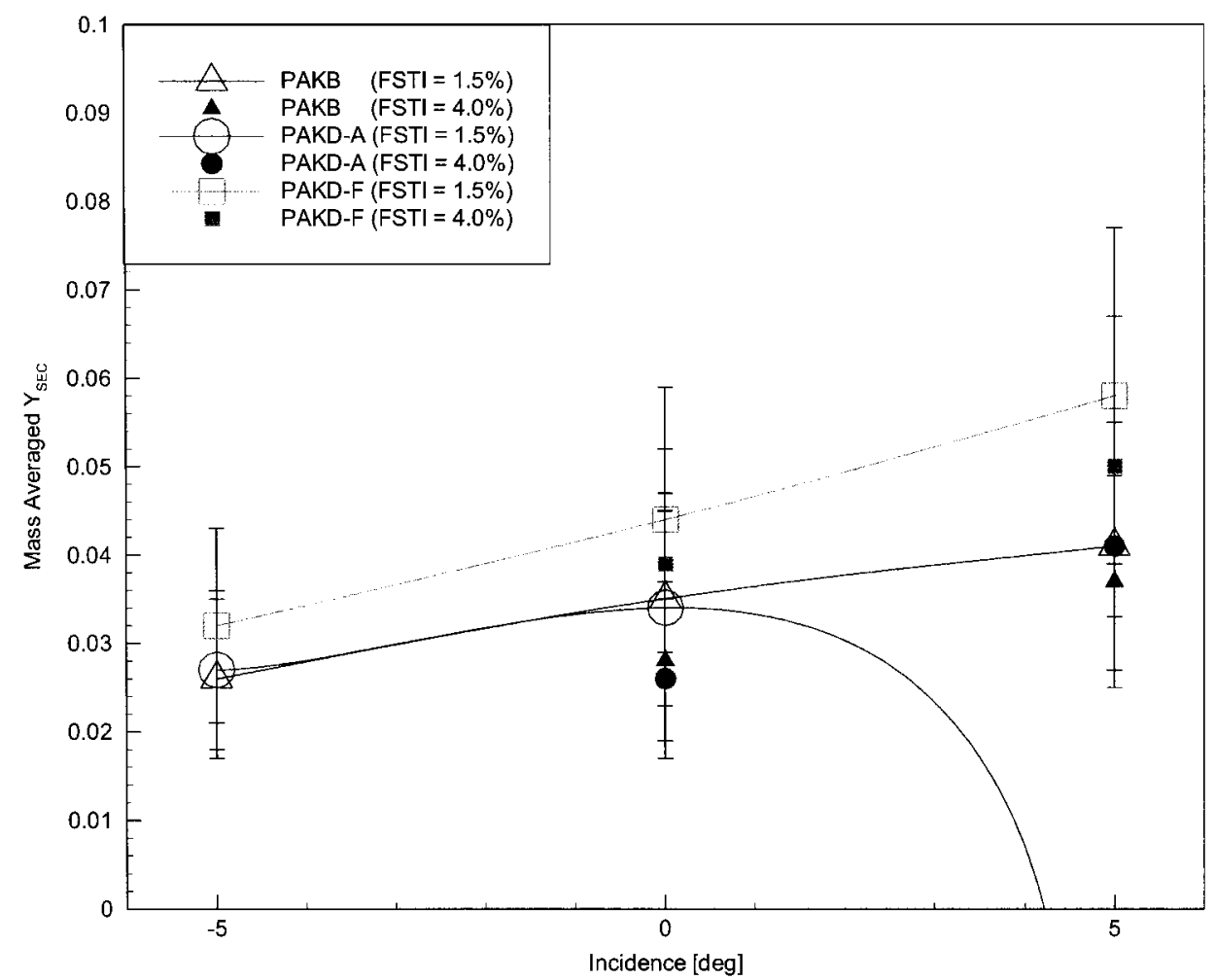

Figure 5.10: Mass-Averaged Secondary Losses at Mixed-Out Plane

As already mentioned in Chapter 4, Popovic (2005) concluded that the PAKD-A is on the verge of stall at Re of 80,000 , FSTI of $1.5 \%$ and design incidence. At $+5^{\circ}$ incidence and the same Reynolds number and FSTI level, Popovic found that the 
Table 5.6: Summary of Mass-Averaged Secondary Losses at Mixed-Out Plane

\begin{tabular}{|c|c|c|c|c|}
\cline { 3 - 5 } \multicolumn{2}{c|}{} & PAKB & PAKD-A & PAKD-F \\
\hline \multirow{3}{*}{ FSTI=1.5\% } & $-5 \mathrm{deg}$ & 0.026 & 0.027 & 0.032 \\
\cline { 2 - 5 } & $0 \mathrm{deg}$ & 0.035 & 0.034 & 0.044 \\
\cline { 2 - 5 } & $+5 \mathrm{deg}$ & 0.041 & -0.223 & 0.058 \\
\hline \multirow{3}{*}{$\mathrm{FSTI}=4.0 \%$} & $-5 \mathrm{deg}$ & \multicolumn{3}{|c}{} \\
\cline { 2 - 5 } & $0 \mathrm{deg}$ & 0.028 & 0.026 & 0.039 \\
\cline { 2 - 5 } & $+5 \mathrm{deg}$ & 0.037 & 0.041 & 0.05 \\
\hline
\end{tabular}

PAKD-A airfoil is stalled. This is evident from the total pressure contours shown in Figure 5.11. The total pressure coefficient contours are distorted and there are no recognizable secondary flow features (such as the bone shaped structure). There is a large area of high total-pressure loss corresponding to the wide blade wake, denoted by blue color.

As a result of the high profile losses seen at midspan (which are subtracted from the total losses to obtain the secondary losses), the secondary losses turn out to be negative for this case. Benner et al. (2004b) also reported a negative value of secondary losses in their measurements at $+20^{\circ}$ incidence. They attributed this result to the conventional loss breakdown, which assumes that profile losses are constant across the span, which is clearly not the case in regions close to the endwall that are influenced by secondary flows. Benner et al. (2005a) proposed a new loss breakdown scheme that accounts for the non-uniformity of the losses generated in the blade surface boundary layers across the span and yields more reasonable values of secondary losses. Both breakdown schemes were introduced in Section 2.3. The new breakdown scheme due to Benner et al. (2005a) will be examined in the following chapter. 

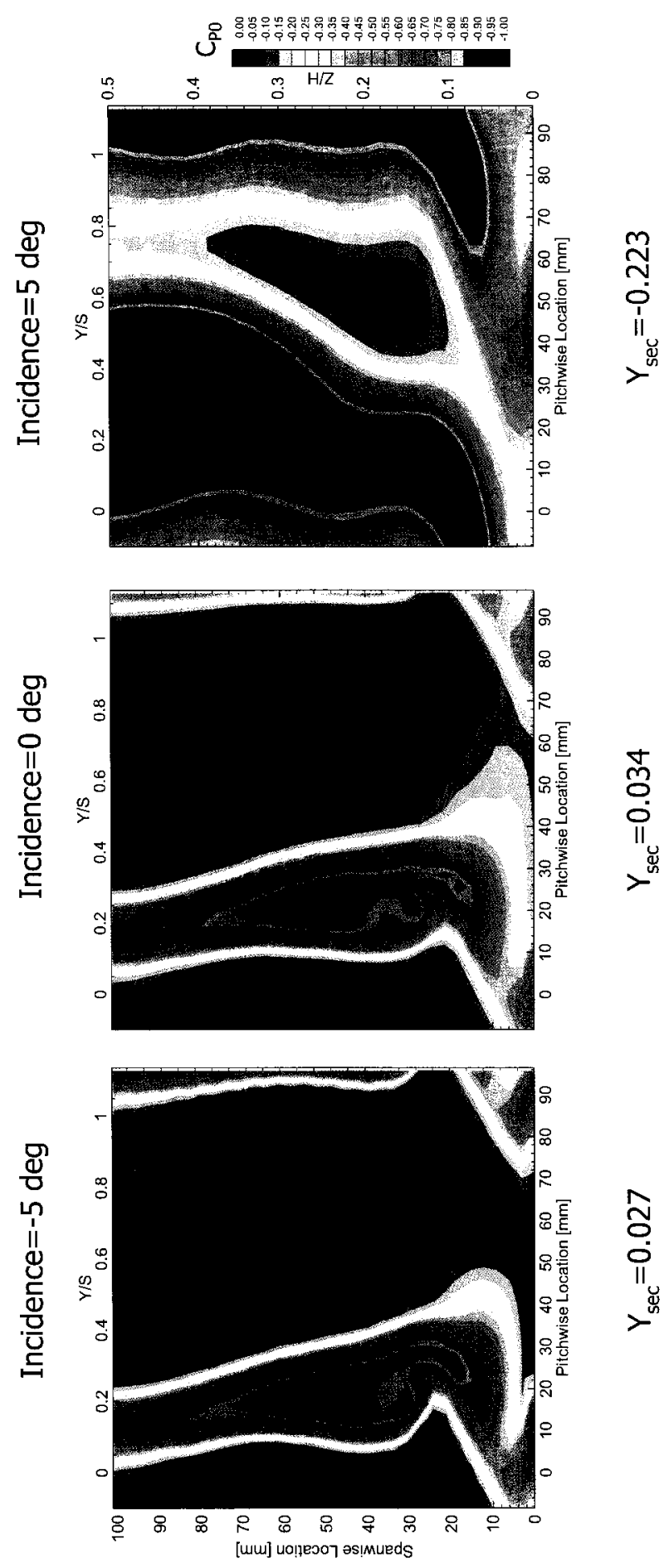

Figure 5.11: PAKD-A: Total Pressure Loss Contours at Design and Off-Design Incidence 
The streamwise vorticity contours for PAKD-A at an incidence of $+5^{\circ}$ and FSTI of $1.5 \%$, are presented in Figures 5.12 and 5.13. They also show that the magnitude and the extent of the vorticity decreased substantially. A stronger effect is seen in the positive vorticity (counter and corner vortices) which is reduced to a few patches of low magnitude. The negative vorticity, associated with the passage vortex, is also reduced in magnitude and pushed towards the endwall. The region of negative vorticity is expanded in the pitchwise direction with the increase in incidence and now covers the full pitch between two adjacent blades. The notable changes in the regions of positive and negative vorticity seem to be associated with the massive twodimensional flow separation (stall) on PAKD-A suction surface at given conditions. To the author's knowledge, none of the previous authors investigating the effect of incidence variation on the secondary losses except Benner et al. (2004b) observed cases experiencing stall. In their flow measurements, Benner et al. (2004b) noticed that in the presence of an open laminar separation bubble on the suction surface (at $+20^{\circ}$ incidence) the strength of the passage vortex did not change compared to the values obtained from the measurements at $+10^{\circ}$. This was not the case when the incidence increased from $0^{\circ}$ to $+10^{\circ}$, where the strength of the passage vortex became greater with increasing incidence. Benner et al. (2004b) argued that the destruction of vorticity and reduction in vortex strength occurred due to the mixing of the two regions of fluid of opposite vorticity. They believed that the mixing of the fluid containing opposite signs of vorticity increases with increasing incidence. Thus, it is also possible that a massive stall, such as one experienced by the PAKD-A airfoil, has had an influence on the destruction of vorticity in that particular case. 

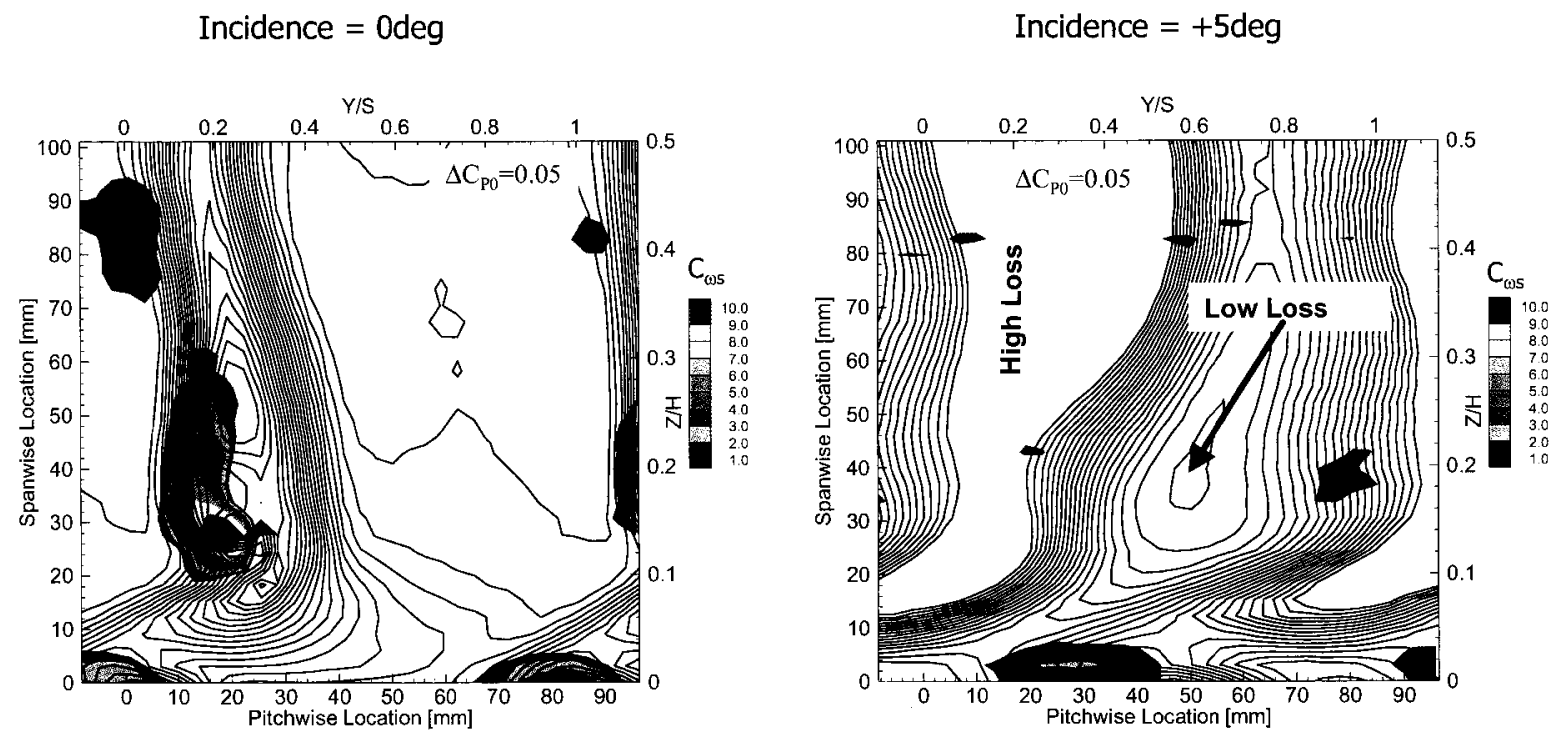

Figure 5.12: PAKD-A: Positive Vorticity Variation With Incidence at FSTI of $1.5 \%$

Incidence $=0 \mathrm{deg}$

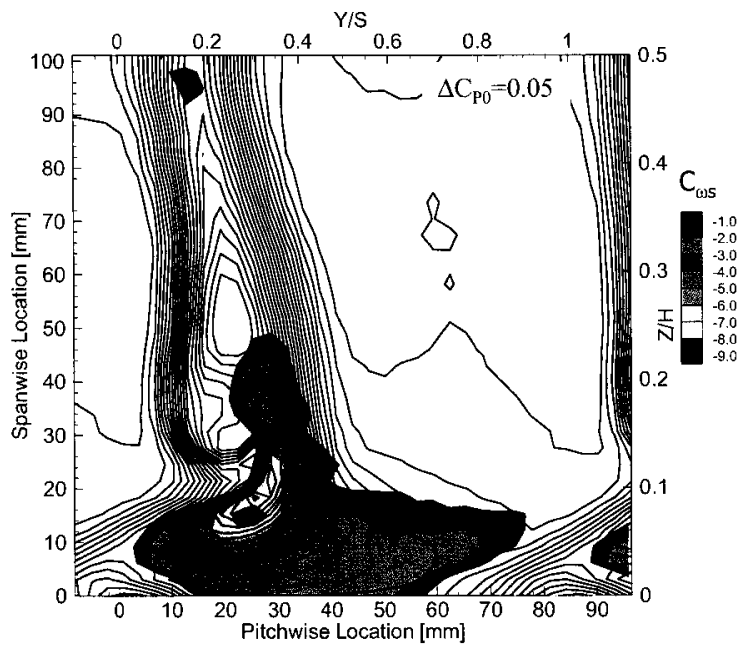

Incidence $=+5$ deg

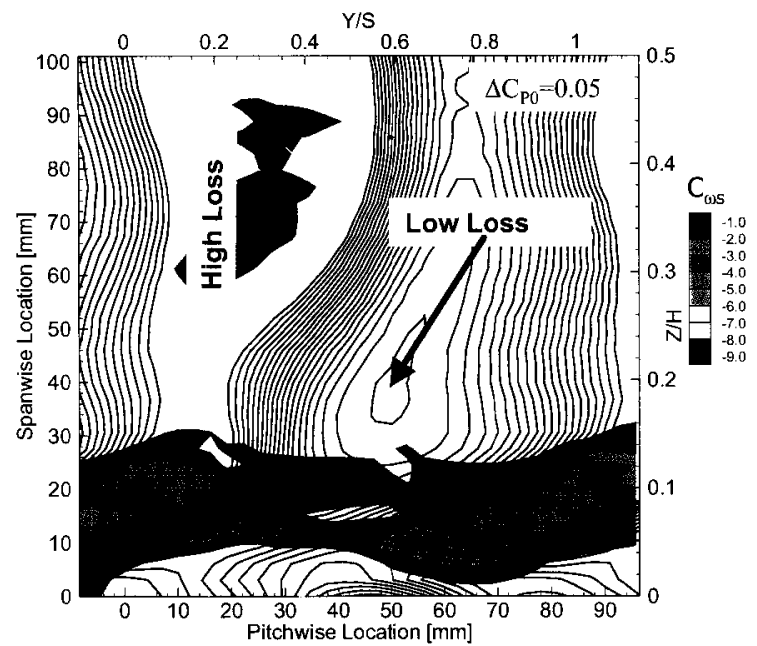

Figure 5.13: PAKD-A : Negative Vorticity Variation With Incidence at FSTI of $1.5 \%$ 


\subsubsection{Axial Velocity Ratio (AVR) Distributions}

Section 5.4.2 showed that the AVR overshoot increases with incidence for the baseline cascade. This was attributed to higher loading on the front part of the airfoil at positive incidence. Except for PAKD-A, which is stalled at $+5^{\circ}$ incidence, the other cases investigated follow the same trend of higher AVR overshoot with increasing incidence. The highest overshoot is seen for the PAKD-F airfoil at $+5^{\circ}$, the case for which the highest strength of the secondary flows is also observed. The maximum measured value of AVR for this case is approximately $40 \%$ higher than the average value. A full set of AVR plots is given in Appendix I.

\subsubsection{The Effect of Varying FSTI}

The effect of increased freestream turbulence on the secondary flows was already discussed in Chapter 4 for the design incidence and Section 5.4.3 of this chapter for the baseline cascade, PAKB, at off-design incidence. Introduction of higher FSTI reduces the magnitude of the secondary losses for PAKB and PAKD-F at all values of incidence, as seen in Figure 5.10, Section 5.5.1. No comparable observation can be made for the PAKD-A airfoil at $+5^{\circ}$ incidence. As was already mentioned, PAKD-A is stalled at FSTI of $1.5 \%$ and $+5^{\circ}$ incidence. The stall results in a negative value of mixed-out mass-averaged losses and destruction of streamwise vorticity. When the FSTI level is raised to 4.0\%, the PAKD-A airfoil is no longer stalled. The total pressure coefficient contours for PAKD-A at $+5^{\circ}$ incidence and the two levels of FSTIs are shown in Figure 5.14. The $C_{P 0}$ contours at the higher FSTI show that a recognizable secondary flow pattern is reestablished for this case. The same can be said for the streamwise vorticity contours presented in Figures 5.15 and 5.16. 
The effect of higher FSTI on the PAKD-F airfoil at off-design incidence was similar to that for PAKB: the total pressure loss coefficient peak magnitudes and the strength of the passage vortex were reduced. This resulted in lower mixed-out mass-averaged secondary losses at higher FSTI.

The full set of plots presenting $C_{P 0}$, positive and negative streamwise vorticity variation with FSTI for all airfoils can be seen in Appendices F, G, and H, respectively.

\subsection{Summary of Conclusions}

This chapter presented and discussed the secondary flow measurements made at off-design incidence for PAKB, PAKD-A, and PAKD-F. Two values of off-design incidence were examined, $-5^{\circ}$ and $+5^{\circ}$. As in the design incidence investigation, the
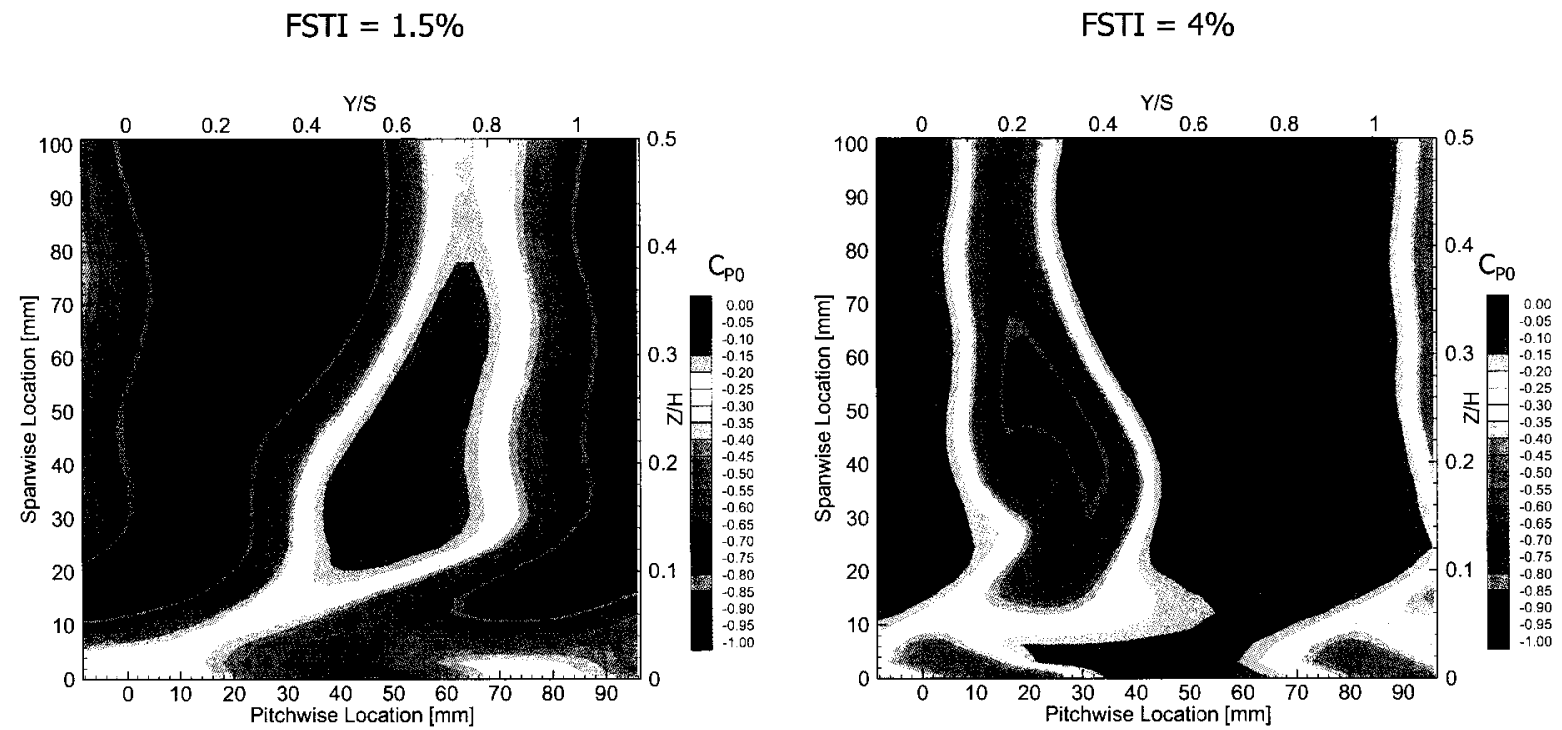

Figure 5.14: PAKD-A: Total Pressure Loss Contours at $+5^{\circ}$ Incidence and FSTI of $1.5 \%$ and $4.0 \%$ 

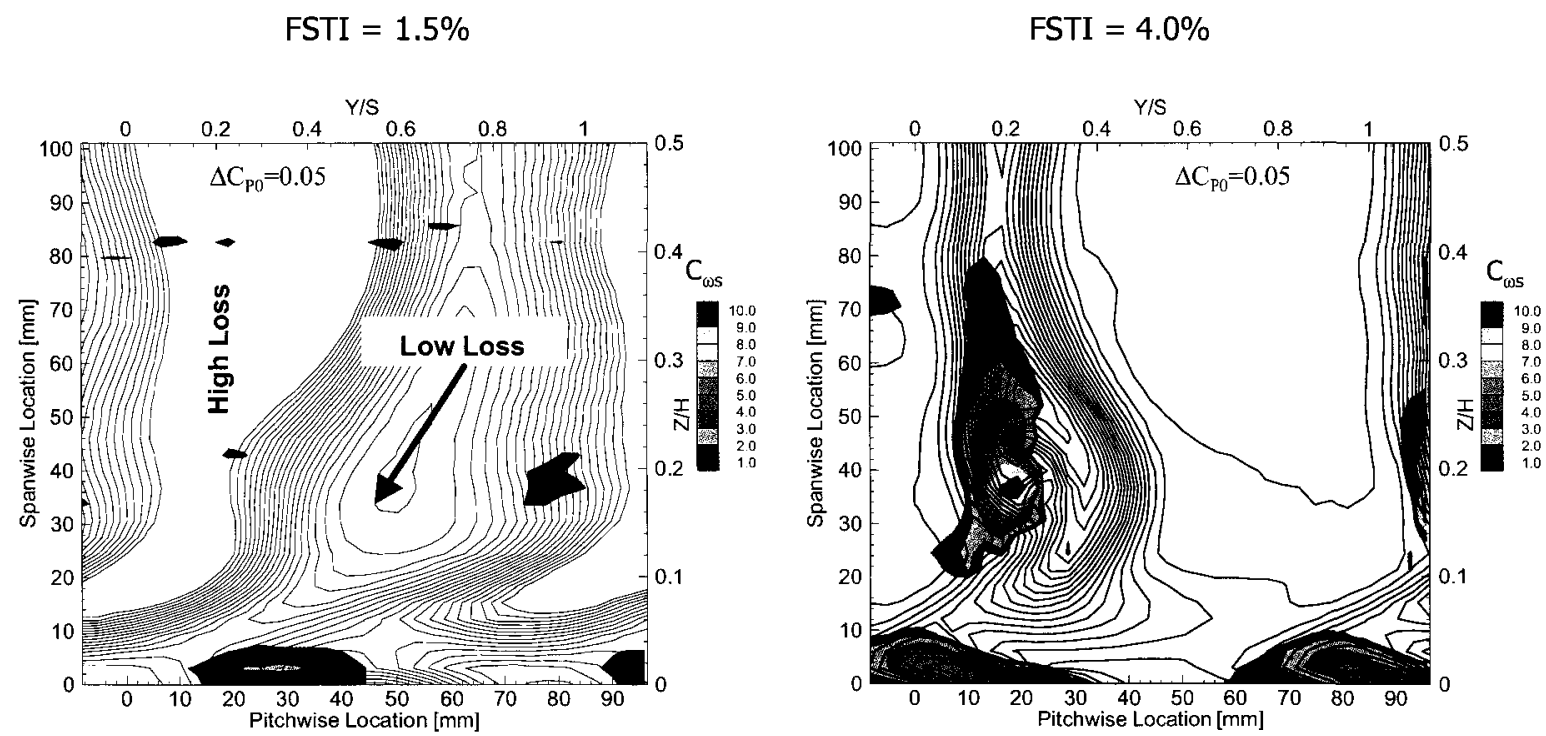

Figure 5.15: PAKD-A: Positive Vorticity Variation With FSTI at $+5^{\circ}$ Incidence
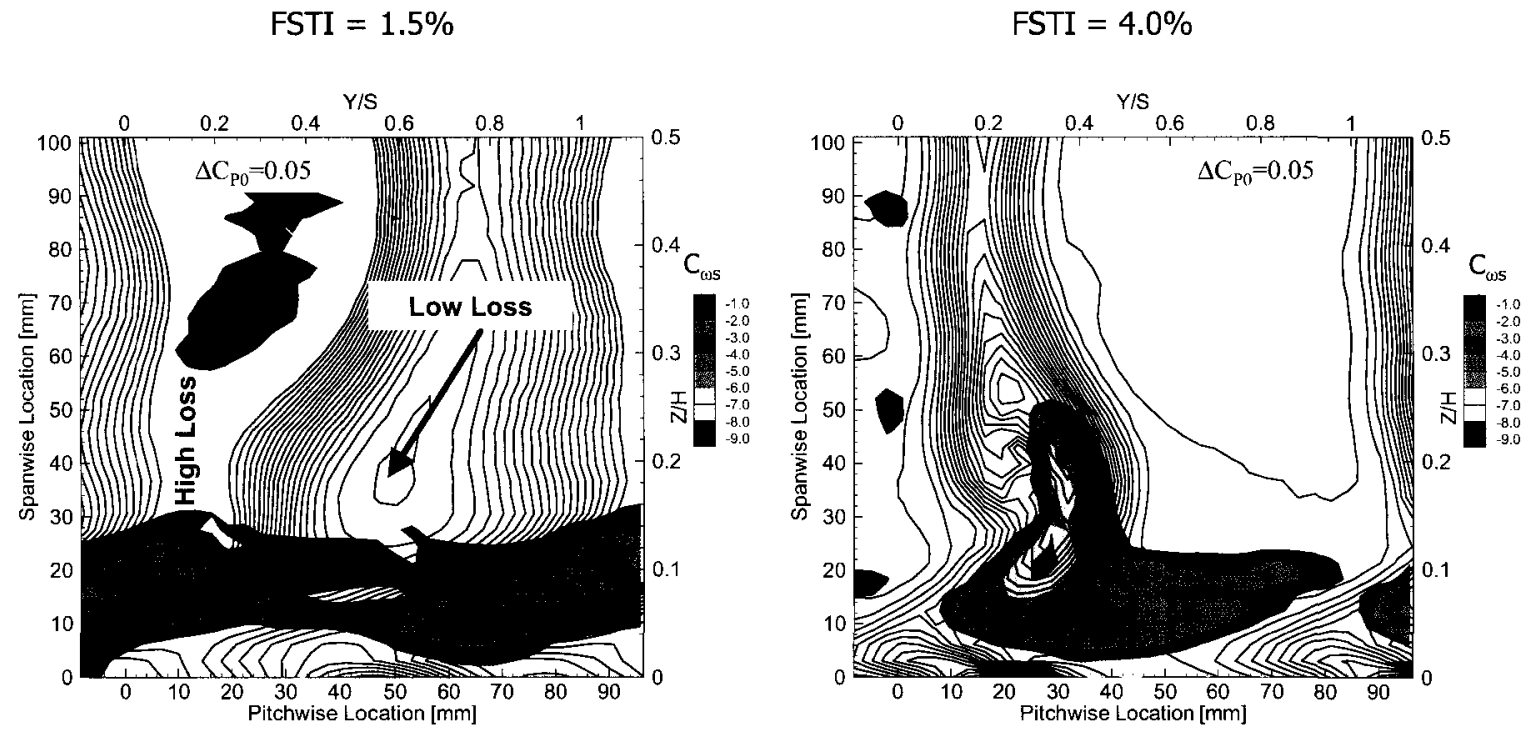

Figure 5.16: PAKD-A : Negative Vorticity Variation With FSTI at $+\mathbf{5}^{\circ}$ Incidence 
measurements were conducted at a constant Reynolds number of 80,000 , and two freestream turbulence intensities of $1.5 \%$ and $4.0 \%$.

The results have shown that the secondary losses, AVR overshoot and the extent and the strength of the passage vortex increase with incidence. With the exception of PAKD-A results at FSTI of $1.5 \%$ and $+5^{\circ}$ (stalled case), the values of mixedout mass-averaged secondary losses for the PAKB and PAKD-A airfoils were again comparable. The PAKD-F airfoil experienced highest secondary losses of all cases investigated.

The PAKD-A stalled when it was subjected to $+5^{\circ}$ incidence at lower FSTI. This resulted in a negative mass-averaged value of the secondary loss coefficient. This negative secondary loss coefficient is a consequence of the way the components of the losses are defined and is not physically meaningful. In addition, a destruction of streamwise vorticity was observed for this case. This was attributed to the mixing of the two regions of fluid of opposite vorticity when the airfoil stalled.

The AVR overshoots increased with incidence. It is believed that this is indirectly due to the increased loading on the front part of the airfoil at positive incidence. Increased loading on the front part of the airfoil increases the strength of the secondary flows in the passage. This results in stronger and larger vortices, which increase the flow blockage. The increased blockage forces the axial velocity up in the area located between the three major vortical structures. The highest overshoot, roughly $40 \%$ higher than the average value of AVR, was detected for the PAKD-F airfoil when it was subjected to positive incidence.

Increasing the FSTI resulted in a decrease in the strength of the secondary flows and the magnitude of the secondary losses. The PAKD-F airfoil had the highest 
secondary losses under all operating conditions.

This concludes the discussion of the results at off-design incidence. The next chapter will evaluate the existing empirical secondary flow correlations and new loss breakdown scheme proposed by Benner et al. (2005a). 


\section{Chapter 6}

\section{Comparison of Measured}

\section{Secondary Losses with Empirical}

\section{Predictions}

\subsection{Introduction}

In the early stages of turbine design, an accurate estimate of the losses is important. Empirical correlations are a useful tool in loss prediction and are widely used by turbine designers. Given the extent of research and the advances in the area of turbine blade design it is necessary to keep up with the progress and to update the correlations so that they can be applied to the new generations of highly loaded airfoils. A brief outline of some of the most known and used secondary flow correlations is provided in Section 6.2. A comparison of measured secondary losses with empirical predictions is presented and discussed in Section 6.3 including an 
evaluation of the new loss breakdown presented recently by Benner et al. $(2005 a)$. Finally, the chapter ends with some concluding remarks.

\subsection{Review of Correlations}

One of the first loss systems for axial turbines was proposed by Ainley and Mathieson (1951). They devised a method for the prediction of the losses at both design and off-design incidence for each of the loss components (profile, secondary, tip leakage, etc). Their secondary loss correlation is expressed as:

$$
Y_{S E C_{A M}}=\lambda Z
$$

where $\lambda$ is given graphically as a function of inlet and outlet annulus geometry and $\mathrm{Z}$ is the loading parameter defined as:

$$
Z=\left(\frac{C_{L}}{s / c}\right)^{2} \frac{\cos ^{2} \alpha_{2}}{\cos ^{3} \alpha_{\text {mean }}}
$$

$\frac{C_{L}}{s / c}$ is the lift coefficient over pitch-to-chord ratio and $\alpha_{m}$ is the angle of the vector mean velocity. They are given as:

$$
\begin{gathered}
\frac{C_{L}}{s / c}=2\left(\tan \alpha_{1}+\tan \alpha_{2}\right) \cos \alpha_{\text {mean }} \\
\alpha_{\text {mean }}=\arctan \left[1 / 2\left(\tan \alpha_{1}-\tan \alpha_{2}\right)\right]
\end{gathered}
$$

where $\alpha_{1}$ and $\alpha_{2}$ are the inlet and outlet flow angles, respectively.

Dunham and Came (1970) updated Ainley and Mathieson's correlation by in- 
cluding the blade aspect ratio as one of the parameters influencing the secondary losses. Blade aspect ratio is defined as ratio of blade span (h) to true chord (c). Dunham and Came assume that the secondary losses vary as the reciprocal of the blade aspect ratio, i.e the secondary loss increases in magnitude as the blade aspect ratio is reduced. The final form of the correlation by Dunham and Came is given as:

$$
Y_{S E C_{A M D C}}=0.0334\left(\frac{c}{h}\right)\left(\frac{\cos \alpha_{2}}{\cos \beta_{1}}\right) Z
$$

where $\mathrm{Z}$ is the same loading parameter defined by Ainley and Mathieson (1951) and $\beta_{1}$ is the inlet blade metal angle.

Expanding on the work of Dunham and Came (1970), Kacker and Okapuu (1982) offered an improved correlation for secondary losses at design incidence. They also recognized the dependence of secondary losses on the blade aspect ratio and verified the trend of a rise in losses with reduction in aspect ratio. However, the Kacker and Okapuu's correlation has the losses increasing less rapidly for blades with aspect ratio of less than 2.0. Therefore, the new prediction method distinguished between blades with aspect ratios greater than or less then 2.0. The final form of the new secondary loss correlation is given by:

$$
Y_{S E C_{A M D C}}=0.0334 f_{(A R)}\left(\frac{\cos \alpha_{2}}{\cos \beta_{1}}\right) Z
$$

where $\mathrm{Z}$ is again the same loading parameter as defined by Ainley and Mathieson (1951) and Dunham and Came (1970) (which will be referred to as "AMDC"). The $f_{(A R)}$ parameter is defined as:

$$
f_{(A R)}=\frac{1-0.25 \sqrt{2-h / c}}{h / c} \text { for } h / c \leq 2
$$




$$
f_{(A R)}=\frac{1}{h / c} \quad \text { for } \quad h / c>2
$$

It should also be noted that in the AMDC prediction method the secondary loss $\left(Y_{S E C_{A M D C}}\right)$ is multiplied by the magnitude of trailing edge losses, while the Kacker and Okapuu correlation (which will be referred to as K-O) does not link the secondary and trailing edge losses. In order to relate the secondary loss prediction systems of AMDC and K-O it is necessary to scale the $Y_{S E C_{A M D C}}$ such that:

$$
Y_{S E C_{K O}}=1.2 Y_{S E C_{A M D C}} K_{s}
$$

The $K_{s}$ is the subsonic Mach number correction and is given by:

$$
K_{s}=1-\left(\frac{1}{h / C_{x}}\right)^{2}\left(\frac{M_{1}}{M_{2}}\right)^{2}\left[1.25\left(M_{2}-0.2\right)\right]
$$

where $\mathrm{h}$ is the blade span, $C_{x}$ the axial chord, and $M_{1}$ and $M_{2}$ are the inlet and outlet Mach numbers respectively. Subsonic Mach number correction takes a value of 1.0 for the incompressible flow, as is the case in this investigation.

Expanding on the preceding secondary loss prediction methods, Sharma and Butler (1987) proposed a new semi-empirical approach for correlating secondary losses. They were the first to identify another parameter, the penetration depth. The penetration depth, discussed in Section 2.2.2, is a measure of the diameter of the passage vortex and thus the spanwise extent of the secondary flow. The correlation 
for penetration depth due to Sharma and Butler is given as follows:

$$
\frac{Z_{T E}}{C_{x}}=0.15 \frac{\varepsilon}{\sqrt{C R}}+f\left(\frac{\delta_{1}}{h}\right)
$$

where:

$$
\begin{array}{ll}
Z_{T E}: & \text { Penetration depth at trailing edge } \\
\varepsilon: & \text { Gas turning in radians } \\
C R: & \text { Convergence ratio }\left(\frac{\rho_{2} V_{2}}{\rho_{1} V_{1}}\right) \\
\delta_{1}: & \text { Inlet boundary layer thickness } \\
h: & \text { Blade height (span) }
\end{array}
$$

and $f\left(\frac{\delta_{1}}{h}\right)$ is expressed as:

$$
f\left(\frac{\delta_{1}}{h}\right)=1.4\left(\frac{\delta_{1}}{h}\right)-2.73\left(\frac{\delta_{1}}{h}\right)^{2}+1.77\left(\frac{\delta_{1}}{h}\right)^{3}
$$

Finally, the new endwall loss prediction is given by:

$$
\left(1-\phi^{2}\right)_{S E C}=\left(1-\phi^{2}\right)_{2 D} \frac{s \sin ^{2} \beta_{2}-T E T}{h}\left(1+4 \frac{\varepsilon}{\sqrt{C R}}\right)
$$

where:

$$
\begin{array}{ll}
\left(1-\phi^{2}\right)_{S E C}: & \text { Endwall (secondary) energy loss coefficient } \\
\left(1-\phi^{2}\right)_{2 D}: & \text { Two dimensional energy loss coefficient } \\
s: & \text { Blade pitch } \\
\text { TET : } & \text { Trailing edge thickness }
\end{array}
$$


Using equation 2.5 given in Section 2.2.2 it is possible to relate the endwall energy loss coefficient given by Sharma and Butler to the total pressure loss coefficient $Y_{S E C}$ as follows:

$$
Y_{S E C_{S B}}=\frac{1}{\left(1-\phi^{2}\right)_{S E C}}-1
$$

More recently Moustapha et al. (1990) reviewed the existing correlations for profile and secondary losses at off-design incidence and concluded that further improvements were required. They offered an improved correlation for the secondary losses that incorporates the airfoil leading edge diameter and degree of acceleration in the blade channel as correlating parameters. Although not included in the correlation, Moustapha et al. (1990) also discussed the influence of other parameters on the losses: Mach number, Reynolds number, turbulence level, and exit flow angle, to name a few. The new correlation is meant to be used in conjunction with the Kacker and Okapuu correlation (which is applicable for design incidence only) and is presented as follows:

$$
\begin{array}{ll}
\left(\frac{Y}{Y_{d e s}}\right)_{S E C_{M}}=\exp ^{\left(0.9 X^{\prime \prime}\right)}+13 X^{\prime 2}+400 X^{\prime \prime 4} & \text { for } 0<X^{\prime \prime}<0.3 \\
\left(\frac{Y}{Y_{d e s}}\right)_{S E C_{M}}=\exp ^{\left(0.9 X^{\prime \prime}\right)} & \text { for }-0.4<X^{\prime \prime}<0
\end{array}
$$

where:

$$
X^{\prime \prime}=\frac{\alpha_{1}-\beta_{1}}{180-\left(\beta_{1}+\beta_{2}\right)}\left(\frac{\cos \beta_{1}}{\cos \beta_{2}}\right)^{-1.5}\left(\frac{d}{c}\right)^{-0.3}
$$

and $\mathrm{d}$ is the airfoil leading edge diameter, $\mathrm{c}$ is the true chord.

The most recent secondary loss correlation known to the author was proposed by Benner et al. (2005b). Based on an expanded database of experimental cases Benner et al. $(2005 a)$ were also able to update the penetration depth correlation by including the airfoil loading term as one of the correlating parameters. The chosen loading 
parameter $\left(F_{t}\right)$ represents the tangential loading on the airfoil and is given by:

$$
F_{t}=2\left(\frac{s}{C_{x}}\right) \cos ^{2} \alpha_{m}\left(\tan \alpha_{1}-\tan \alpha_{2}\right)
$$

The definition of the angle of the vector mean velocity $\left(\alpha_{m}\right)$ was given in equation 6.2. The updated correlation for penetration depth due to Benner et al. (2005a) is:

$$
\frac{Z_{T E}}{h}=\frac{0.1 F_{t}^{0.79}}{\sqrt{C R}\left(\frac{h}{C}\right)^{0.55}}+32.70\left(\frac{\delta^{*}}{h}\right)^{2}
$$

It should be noted that instead of the inlet boundary layer thickness used by Sharma and Butler in equation 6.6, Benner et al. (2005a) use the inlet boundary layer displacement thickness $\left(\delta^{*}\right)$ in the last term of their penetration depth correlation.

Recognizing the dependance of the secondary losses on the airfoil aspect ratio Benner et al. (2005b) define separate expressions applicable to low $\left(\frac{h}{c} \leq 2\right)$ and high $\left(\frac{h}{c}>2\right)$ aspect ratio blades as follows:

$$
\begin{aligned}
& Y_{S E C_{B}}=\frac{0.038+0.41 \tanh \left(1.20 \delta^{*} / h\right)}{\sqrt{\cos \gamma}(C R)\left(\frac{h}{C}\right)^{0.55}\left(\frac{C \cos \alpha_{2}}{C_{x}}\right)^{0.55}} \quad \text { for } \quad\left(\frac{h}{c} \leq 2\right) \\
& Y_{S E C_{B}}=\frac{0.052+0.56 \tanh \left(1.20 \delta^{*} / h\right)}{\sqrt{\cos \gamma}(C R)\left(\frac{h}{C}\right)\left(\frac{C \cos \alpha_{2}}{C_{x}}\right)^{0.55}} \quad \text { for } \quad\left(\frac{h}{c}>2\right)
\end{aligned}
$$

where $\gamma$ is the stagger angle.

The Benner at al. correlation is meant to be used with the new loss breakdown scheme discussed in Section 2.3 and is applicable at both design and off-design values of incidence. 


\subsection{Evaluation of Correlations}

\subsubsection{Introduction}

Three of the previously mentioned secondary loss prediction methods, namely Kacker and Okapuu (1982), Moustapha et al. (1990) and Benner et al. (2005b) will be compared with the experimental data obtained by the present author.

As mentioned in the previous section, the correlation of both Kacker and Okapuu and Moustapha et al. use the inlet metal angle as one of the correlating parameters. In addition, Moustapha et al. also use the leading-edge circle diameter in their correlation. However, it is often the case that leading-edge circles are not employed in the design of modern airfoils. This is true for the PAKB and PAKD airfoils used in the present investigation. Therefore, for the purpose of evaluation of these correlations it was assumed that the inlet metal angle $\left(\beta_{1}\right)$ is equal to the inlet flow angle $\left(\alpha_{1}\right)$ at design conditions and the approximate "leading edge diameters" were estimated based on the airfoil geometries in the leading edge region.

The previous section noted that the Benner et al. correlation is meant to be used with a new loss breakdown scheme. The following section will briefly compare the secondary loss coefficients obtained from the conventional and new loss breakdown schemes.

\subsubsection{New Loss Breakdown Scheme}

The new loss breakdown scheme was introduced in Section 2.3. Benner et al. (2005a) found that the conventional loss breakdown does not yield physically mean- 
ingful magnitudes of secondary flow coefficient when blades are subjected to high positive values of incidence and the flow in the primary regions may be separated. The new loss breakdown scheme clearly distinguishes the profile losses as those generated in the primary flow region only and is defined as:

$$
Y_{T O T A L}=Y_{P}+Y_{S E C}=Y_{M I D S P A N}\left(1-\frac{Z_{T E}}{h}\right)+Y_{S E C}
$$

where $Z_{T E}$ is the penetration depth of the secondary flow and $h$ is the blade height. Since flow visualization was not performed for the current measurements, the penetration depth was not directly available. Instead, it was determined based on equation 6.9. The values of penetration depth for the three cascades at design incidence and both values of FSTI examined are given next.

Figures 6.1 and 6.2 show the comparison between secondary loss coefficients obtained using the conventional and new loss breakdown schemes for FSTI values of $1.5 \%$ and $4.0 \%$. The losses obtained using the new loss breakdown are in general higher than those due to the conventional breakdown. The biggest difference between the two loss breakdowns is observed for the PAKD-A airfoil at $+5^{\circ}$ incidence and FSTI of 1.5\%. As discussed in Chapter 5, the PAKD-A airfoil is stalled at these particular conditions, and as a result of the high midspan losses, the secondary losses are negative for this case for both loss breakdown schemes. Benner et al. (2004b) also reported an example of negative value of secondary loss coefficient in their measurements at high positive incidence when using the conventional loss breakdown. The new loss breakdown they proposed yielded more physically reasonable (positive) values of secondary loss coefficient for that particular case.

It is believed that there are at least two possible reasons for the negative value of secondary loss coefficient obtained from the new loss breakdown for the present 


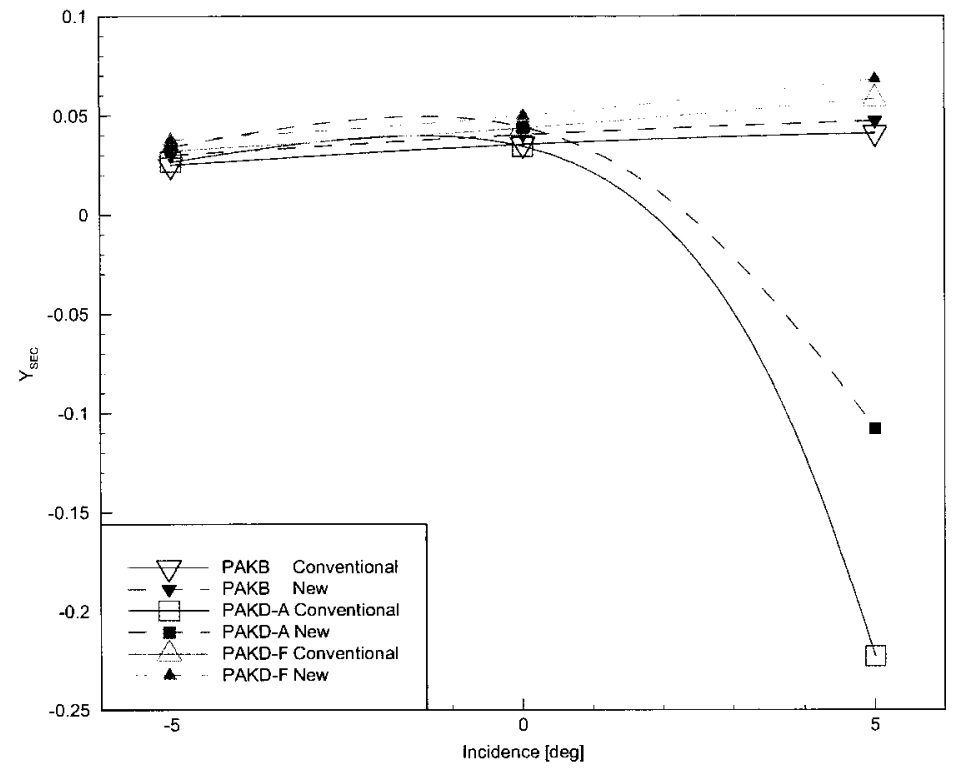

Figure 6.1: Comparison of Secondary Losses for the Three Cascades Using Conventional and New Loss Breakdown Schemes at FSTI=1.5\%

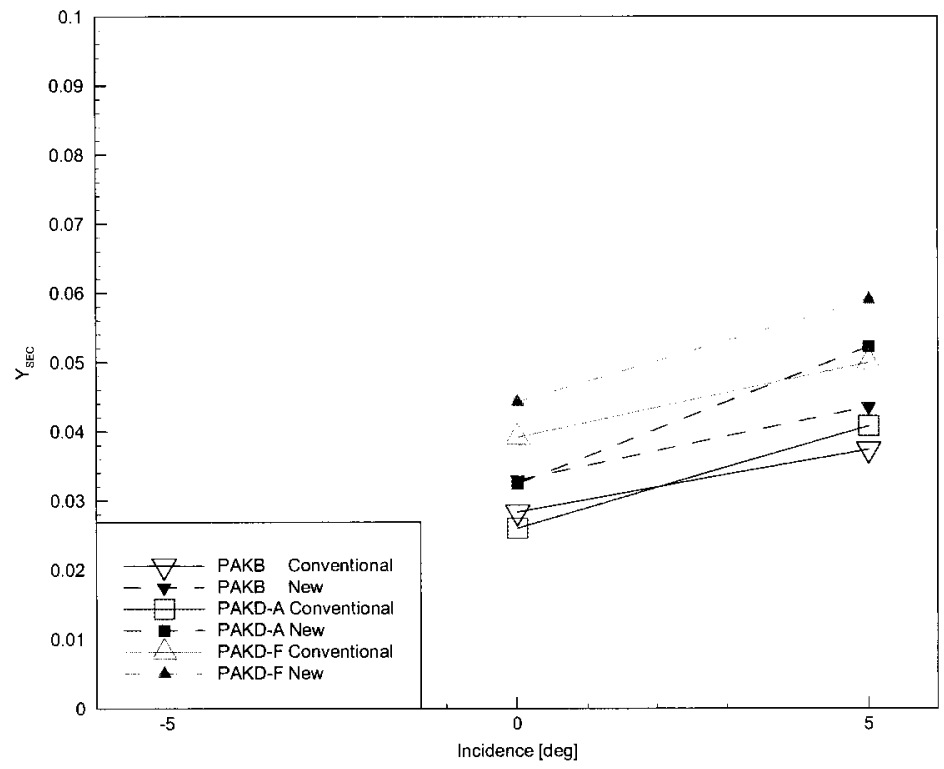

Figure 6.2: Comparison of Secondary Losses for the Three Cascades Using Conventional and New Loss Breakdown Schemes at FSTI $=4.0 \%$ 
measurements. The first lies in the fact that the loss breakdown was calculated based on Benner's et al. correlation for the penetration depth and not its actual value, which was not directly available due to lack of flow visualization during the experiment. Secondly, at $+5^{\circ}$ incidence and FSTI of $1.5 \%$ the PAKD-A is experiencing a massive stall at midspan with flow separation at about $0.5 C_{X}$. As shown in Figure 5.11 , the width of the wake varies noticeably along the span resulting in non-uniform profile loss generation. Thus, for this particular case the profile losses at the midspan may not be representative of the losses generated in the full primary region, as was assumed by Benner et al. (2004b) when developing the new loss breakdown scheme.

\subsubsection{Evaluation at Design Incidence}

Table 6.1 presents the measured and predicted values of the secondary loss coefficients at design incidence for all three airfoils. The values predicted by the correlations of Kacker and Okapuu and Benner et al. are compared with the measured values obtained through the conventional and new loss breakdowns.

Qualitatively the correlations examined show a trend similar to that seen from the experimental data. Both correlations predict similar magnitudes of the losses for PAKB and PAKD-A airfoils at design incidence. Approximately $10 \%$ higher losses are predicted for the PAKD-F airfoil.

The Benner et al. correlation under-predicts the losses for all three cascades. On the other hand, the magnitudes of losses predicted by $\mathrm{K}-\mathrm{O}$ are higher than the measured values. However, it should be noted that $\mathrm{K}-\mathrm{O}$ correlation is intended to predict the losses for the real engine environment, which are known to be significantly higher than those measured in cascades. Thus, to be able to compare the results 
Table 6.1: Summary of Measured and Correlated Secondary Losses for the Three Cascades at Design Incidence

\begin{tabular}{|c|c|c|c|c|c|c|}
\hline & \multicolumn{2}{|c|}{ PAKB } & \multicolumn{2}{|c|}{ PAKD-A } & \multicolumn{2}{|c|}{ PAKDF } \\
\hline & $15 \%$ & $4.0 \%$ & $1.5 \%$ & $40 \%$ & $15 \%$ & $4.0 \%$ \\
\hline $\begin{array}{l}Y_{\text {SEC }} \text { Measured Conventional } \\
\text { Loss Breakdown }\end{array}$ & 0.035 & 0.028 & 0.034 & 0.026 & 0.044 & 0.039 \\
\hline Kacker \& Okapuu (1982) & \multicolumn{2}{|c|}{0.067} & \multicolumn{2}{|c|}{0.068} & \multicolumn{2}{|c|}{0.075} \\
\hline $\begin{array}{l}\text { Kacker } \& \text { Okapuu (1982) - } \\
\text { multiplied by a factor of } 0.23\end{array}$ & \multicolumn{2}{|c|}{0.015} & \multicolumn{2}{|c|}{0.016} & \multicolumn{2}{|c|}{0.017} \\
\hline $\begin{array}{l}Y_{\text {SEC }} \text { Measured } \\
\text { New Loss Breakdown }\end{array}$ & 0.040 & 0.033 & 0.044 & 0.032 & 0.050 & 0.044 \\
\hline Benner et al (2005) & 0.024 & 0.021 & 0.024 & 0.021 & 0.027 & 0.023 \\
\hline Penetration Depth $\left(\mathrm{Z}_{\mathrm{TE}} / \mathrm{h}\right)$ & 0.139 & 0.128 & 0.164 & 0.153 & 0.172 & 0.161 \\
\hline
\end{tabular}

obtained from the correlation with experimental data from the cascade, an engineto-cascade conversion factor needs to be applied. Benner et al. (2005b) suggested that applying a factor of 0.23 to the secondary loss coefficients obtained from $\mathrm{K}-\mathrm{O}$ correlation yields results that are more applicable to those for cascades. The factor of 0.23 was based on a database containing 34 previously recorded cases. Even with the factor applied, the present results remained outside the error band $( \pm 0.0075)$ of the correlation.

Neither of the correlations includes free stream turbulence intensity as one of the correlating parameters. The difference in secondary loss coefficients for the two FSTI levels predicted by Benner et al. is the result of differences in inlet boundary layer thickness for the two cases. As discussed in Section 4.3, elevated FSTI levels were achieved using turbulence generating grids. The two turbulence generating grids have different geometries and are mounted at two different locations. As a consequence, 
the inlet boundary layer thicknesses are different for the measurements performed at FSTI of $1.5 \%$ and $4 \%$. The boundary layer is almost twice as thick for the lower FSTI grid, since this grid is installed farther upstream of the test cascades and thus the boundary layer develops over a longer distance. The correlation of Benner et al. takes into account the inlet boundary layer displacement thickness. As a result, the predicted secondary losses for the higher FSTI are about $14 \%$ lower. As reported in Chapter 4, a reduction in secondary losses for the cases tested at higher FSTI may be in part due to the higher turbulence intensity and in part due to the thinner boundary layer. Therefore, it is suggested that this issue should receive more attention in order to fully understand the effects of inlet boundary layer thickness and FSTI on secondary loss generation.

\subsubsection{Evaluation at Off-Design Incidence}

Figures 6.3 and 6.4 compare current measurements with the secondary loss correlations of Moustapha et al. (1990) and Benner et al. (2005b) for off-design values of incidence. The secondary losses at off-design incidence are presented relative to the losses at design incidence $\left(Y_{S E C} / Y_{S E C_{d e s}}\right)$. It should be noted that the losses for the stalled case are plotted on the axes along with values of their corresponding magnitudes.

The Moustapha et al. correlation predicts roughly $20 \%$ higher losses for cases subjected to positive incidence and a decrease in losses at negative incidence of about $8 \%$ for all three airfoils. Since the FSTI effect is not included in the Moustapha et al. correlation, the predicted losses are the same for both turbulence intensities. Compared to the correlation, the present experimental data show higher sensitivity to incidence. 


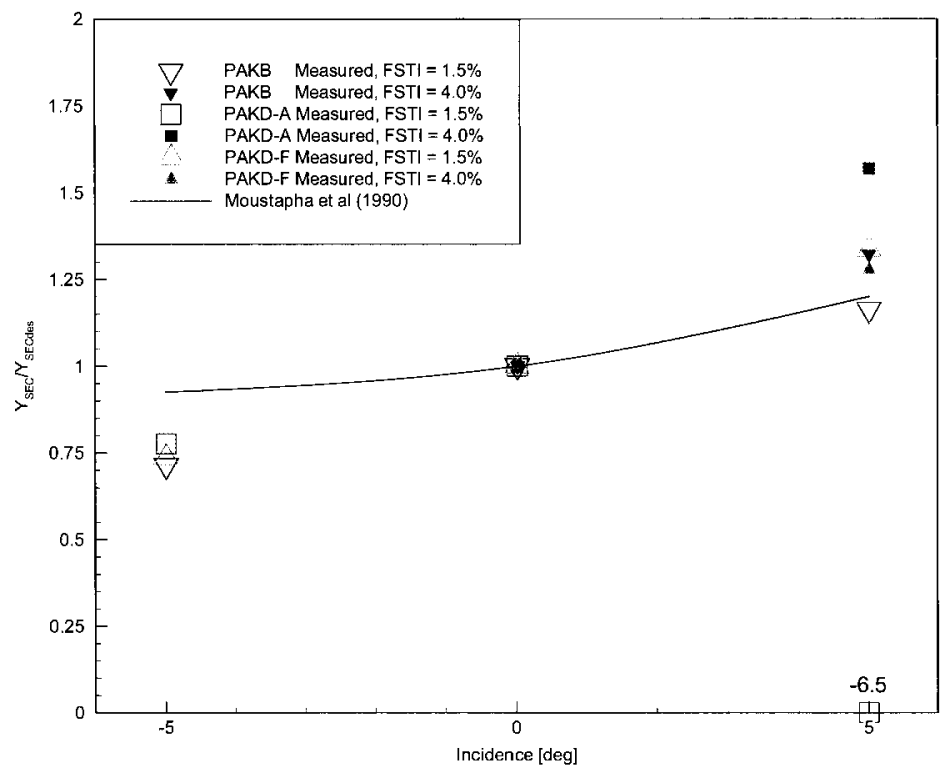

Figure 6.3: Evaluation of Correlation due to Moustapha et al. (1990) (Measured Values Obtained using Conventional Loss Breakdown Scheme)

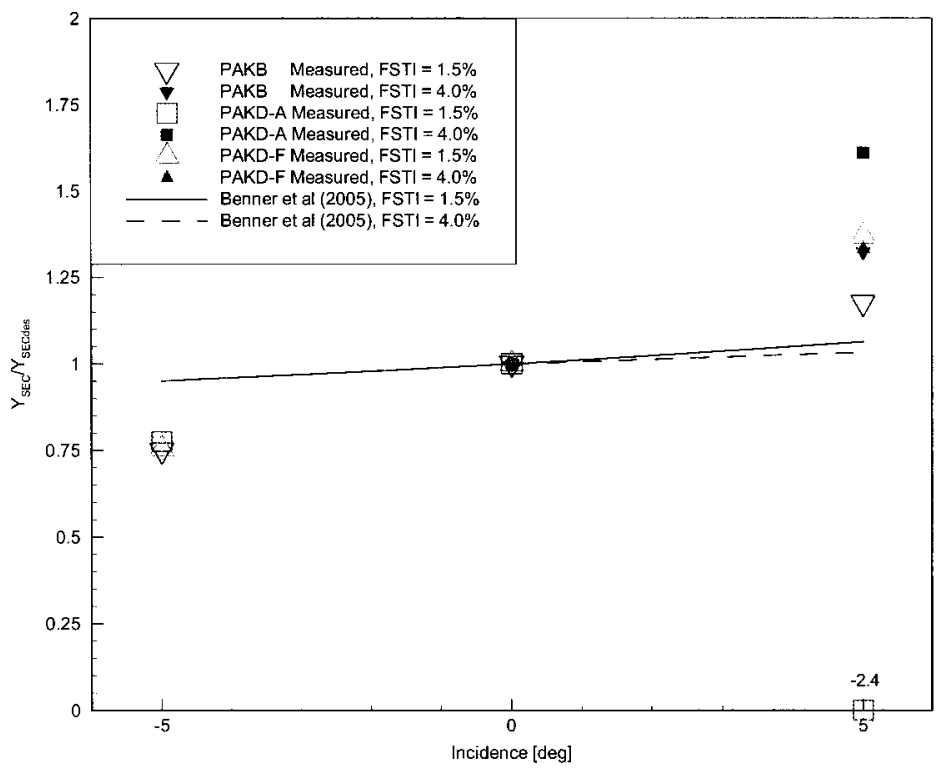

Figure 6.4: Evaluation of Correlation due to Benner et al. (2005b)

(Measured Values Obtained using New Loss Breakdown Scheme) 
The Benner et al. correlation predicts an increase in secondary losses of about $6 \%$ at positive incidence and about $5 \%$ decrease in losses for the negative incidence, compared with the design incidence losses. As mentioned in the previous section, the difference in losses predicted by the correlation for the two FSTI levels is due to the different thicknesses of the inlet boundary layers.

The agreement between experimental and predicted values can be regarded as poor. Neither of the evaluated correlations predicts a noticeable difference in secondary loss trends between three airfoils. It can be clearly seen that the experimental data show much higher sensitivity to the changes in incidence than predicted by the correlations. Therefore, there is a need to revisit the correlations in the future.

\subsection{Concluding Remarks}

This chapter has provided an overview of the main available secondary loss correlations and presented a comparison of the correlations of Kacker and Okapuu (1982), Moustapha et al. (1990), and Benner et al. (2005b) with the present experimental data. The Kacker and Okapuu (1982) correlation is applicable for design incidence, while the Moustapha et al. (1990) correlation applies for off-design incidence only. The most recent correlation due to Benner et al. (2005b) can be applied for both design and off-design conditions. The latter is supposed to be used in conjunction with a new loss breakdown system proposed by the same authors. Hence, the new loss breakdown scheme was also evaluated for the current measurements.

Both the $\mathrm{K}-\mathrm{O}$ and Benner et al. correlations predicted qualitatively similar trends in secondary losses for the three airfoils at design incidence as those seen from the present measurements. The predicted secondary loss magnitudes for the PAKB and 
PAKD-A cascades were practically the same, while both correlations predicted higher secondary losses for the PAKD-F cascade. Quantitatively Benner et al. underpredicted while $\mathrm{K}-\mathrm{O}$ over-predicted the secondary losses at design incidence. The latter was due to the fact that $\mathrm{K}-\mathrm{O}$ is intended for the real engine environment where higher secondary losses are present compared with cascades. Application of a scaling factor proposed by Benner et al. to secondary losses estimated using $\mathrm{K}-\mathrm{O}$ correlation did not bring the losses within the error band of the correlation $( \pm 0.0075)$.

Moustapha et al. and Kacker and Okapuu did not show any difference between the cases at different FSTI. A correct trend was captured by the Benner et al. correlation, but it was due to the thinner inlet boundary layer, not directly due to the FSTI. Therefore, it was suggested that further measurements should be performed to decouple the effects of the inlet boundary layer thickness and turbulence intensity and to incorporate them into future correlations.

The experimental data showed higher sensitivity to the varying incidence than was predicted by both Moustapha et al. and Benner et al. It was concluded that the agreement between the correlations and the current experimental results is poor and that there is a need to revisit the correlations to include the trends exhibited by the highly loaded blades.

The new loss breakdown scheme generally resulted in higher values of secondary loss coefficient. At $+5^{\circ}$ incidence and FSTI of $1.5 \%$, the PAKD-A airfoil was stalled and both conventional and new loss breakdown schemes resulted in negative values of secondary losses. Two possible reasons for this were identified: first, lack of experimentally measured value of penetration depth, and secondly, the non-uniformity of profile loss generation when the airfoil is experiencing a massive flow separation. 


\section{Chapter 7}

\section{Conclusions and Recommendations for Future Research}

\subsection{Conclusions}

Fierce competition between the manufacturers to develop more efficient and cost effective engines has resulted in extensive recent research towards the design of highly loaded compressor and turbine blades. The employment of highly loaded blades in engines is beneficial, because it offers essentially the same component performance with a reduced number of blades. This is achieved through increase in a pitch-tochord ratio between adjacent blades. Low-pressure turbines are one of the heaviest engine components; therefore, it is not surprising that a lot of effort in the industry is aimed at reducing their weight.

The thesis concentrated on an investigation of the losses associated with the secondary flows in three highly loaded low-pressure turbine blade rows. The three 
blades belong to the same family and were designed by Pratt \& Whitney Aircraft for the same duty.

The present research investigated the effects of the loading level and its distribution on the secondary flows. The influence of freestream turbulence intensity and incidence was also examined. The experimental measurements were conducted in an open circuit wind tunnel at a constant Reynolds number of 80,000 and two freestream turbulence intensities (FSTI of $1.5 \%$ and $4.0 \%$ ). Two values of off-design incidence $\left(-5^{\circ}\right.$ and $\left.+5^{\circ}\right)$ were examined.

As expected from the previous investigations, three vortex structures have been identified: the passage, the counter and the corner vortex. Generally, two major loss peaks were observed, one associated with the passage and another with the counter vortex. In some cases a third loss peak was also identified. This peak was not associated with high vorticity. It is believed that the third peak is caused by the twodimensional flow separation from the suction side of the airfoil and that it is induced by the presence of the passage vortex. The axial velocity ratio (AVR) contours have shown that the regions occupied by the vortical structures have lower axial velocity than the average. An overshoot with the maximum value of up to $40 \%$ above the mean value was detected in an area nestled between the two counter-rotating vortices (the passage and the counter vortex), the corner vortex and the projection of the blade trailing edge. This area of higher axial velocity was located in a region of relatively low total pressure loss.

It is generally observed that front-loaded blades have stronger secondary flows. This is mainly due to the stronger cross passage pressure gradients in the channel area in front of the suction peak. The present results have shown that the PAKD-F airfoil indeed has the strongest secondary flows and generates the highest secondary 
losses. In addition, most of researchers agree that the secondary flows, and thus the losses generally increase with the magnitude of the loading. However, the present study shows somewhat different results. The integrated fully mixed-out secondary losses measured for the baseline and the higher loaded airfoil, PAKB and PAKD-A, as obtained using the conventional loss breakdown, essentially had the same magnitude. The presence of separation bubbles on the blade surfaces and their interaction with the secondary flows may lead to an increase in the secondary losses. The loadings taken as part of the present project have shown that there are separation bubbles present on the suction, and possibly the pressure surfaces of the PAKB airfoil. Separation bubbles on the PAKD-A airfoil were present on suction surface only. Thus, it is believed that the higher loss production by the PAKB airfoil may be attributed to stronger interaction of the separation bubbles with the secondary flows present in the passage.

The results have shown that the secondary losses, AVR overshoot and the extent and the strength of the passage vortex increase with incidence. The PAKD-F airfoil generated the highest secondary losses at all examined incidences, while the the losses for the PAKB and PAKD-A blades were comparable. The only exception was for the PAKD-A airfoil at positive incidence and lower FSTI (1.5\%). In that particular case the blade experienced stall. The magnitude of the secondary losses decreased and the secondary flows and vorticity were significantly lower than in the other cases. In fact, the secondary losses in this case were negative, which is physically misleading. This pointed out the problems associated with the traditional method of dividing the losses into secondary and profile losses. Similar results (decrease in secondary losses and vorticity for stalled airfoil) were observed by Benner (2003). The reason for the decrease in secondary losses and vorticity magnitudes was attributed to the massive separation and thus high profile losses combined with the stronger mixing of the two 
regions of fluid of opposite vorticity.

An explanation that the AVR overshoots are indirectly related to the increased loading on the front part of the airfoil at positive incidence was offered. This explanation is supported by the fact that the front-loaded airfoil, PAKD-F, always exhibited higher AVR overshoots. The overshoots for the PAKD-F blade further increased when the airfoil was tested at positive incidence. Subjecting the airfoil to positive incidence increases the loading on the front part of the airfoil, thus suggesting the connection between the AVR overshoots and loading distribution.

Measurements at design and positive incidence have been also performed at higher FSTI. The tests at FSTI of $4.0 \%$ showed that the magnitudes of the losses and the strength of the secondary flows in the passage decreased for all the test cases examined. The "third" loss peak, present in some measurements at lower FSTI disappeared. In addition, the secondary flows were confined to a smaller area and the strength of the vortices associated with them was also decreased. The AVR overshoots were generally reduced with the increase in freestream turbulence level. The trends of increase in the secondary losses with incidence observed at lower FSTI were also applicable for the tests performed at higher FSTI. The losses generated by the PAKD-F airfoil were always higher than those for the other two blades.

It should be noted that turbulence generating grids were used to achieve the different values of FSTI. The inlet boundary layer (IBL) development is affected by the different geometries and mounting positions of the grids. The IBL was twice as thick when the lower FSTI grid was employed. A number of researchers have shown that both the boundary layer thickness and the FSTI can influence the development of the secondary flows. Thus, the above observations and trends might be in part due to the difference in the inlet boundary layer thicknesses and in part due to the 
differences in FSTI.

The present work compared secondary loss correlations due to Kacker and Okapuu (1982), Moustapha et al. (1990), and Benner et al. (2005b) with the results obtained from the current measurements. A new loss breakdown due to Benner et al. (2005a) was also evaluated.

All correlations correctly predicted the qualitative trends in the secondary losses. Kacker and Okapuu (1982) over predicted the losses, while Moustapha et al. (1990), and Benner et al. (2005b) under predicted them. However, for the latter two correlations the sensitivity of the correlated data with varying incidence was smaller than that for the experimental data. A reduction in the secondary losses for the cases with higher FSTI was captured only by Benner et al. (2005a). However, the observed trend was due to the thinner inlet boundary layers and not the FSTI (since the FSTI is not one of the correlating parameters). It was thus concluded that the agreement between the correlations and the current experimental results was generally poor and that there was a need to revisit the correlations to include the quantitative trends exhibited by the highly loaded blades.

Evaluation of the new loss breakdown scheme proposed by Benner et al. (2005a) yielded generally higher values of secondary loss coefficient than when they were evaluated using the conventional loss breakdown. However, even with the new loss breakdown scheme the secondary losses for the stalled case (PAKD-A at $+5^{\circ}$ incidence and FSTI of $1.5 \%$ ) remained negative. Possible explanations were found in the unavailability of the experimentally measured value of penetration depth, and the spanwise non-uniformity of profile loss generation for the stalled case. 


\subsection{Recommendations for Future Work}

The current experimental investigation highlighted several areas of the interest that require further attention.

The present author is of the opinion that flow visualization could be used to develop a better understanding of the physics behind the results observed during the current study. In particular, it is believed that flow visualization by means of smoke or measurements performed by either PIV or LDV systems will be able to give a better idea of the origins of the AVR overshoots observed. In addition, measurements closer to the blade trailing edges as well as within the blade passage could provide information regarding the development of the secondary flow structures as well as the overshoots in the AVR.

It was seen in the thesis that the interaction of the separation bubbles with the secondary flow structures may be responsible for the similar magnitudes of the secondary losses for the PAKB and PAKD-A blades. More information concerning the presence and the size of the separation bubbles could be obtained by using oil and dye flow visualization. In addition, the oil and dye flow visualization may provide support for the proposed explanation of the physics behind the "third" loss peak and would have enabled a better evaluation of the new loss breakdown system proposed by Benner et al. (2005a) through experimental determination of the penetration depth.

The questions raised concerning the influence of the FSTI and IBL thickness on the secondary flows and losses could be answered by looking at these two phenomena separately. It is suggested to perform more tests with varying IBL for several values of FSTI. 
The present measurements have shown that the PAKD-F produces the highest secondary losses of the three cascades. This was expected based on the previous studies which also identified that the front-loaded blades generate higher secondary losses than their aft-loaded counterparts. The picture however changes when looking at the profile losses for the family, where the PAKD-F airfoil turned out to be the best performer offering a superior performance compared to the two other blades. Popovic (2005) reported that the increase of $25 \%$ in loading for PAKD-F compared to the baseline airfoil, PAKB, was achieved with almost no penalty in the profile losses. The ability to decrease the magnitude of the secondary losses would improve the overall aerodynamic performance of the PAKD-F blade. One way to achieve this might be through techniques such as the endwall contouring or airfoil leading edge modifications. These methods may be worth investigating since they would mitigate the high secondary loss generation of the front-loaded airfoil, while still keeping the profile losses low. 


\section{References}

Adjlout, L. and Dixon, S. L. (1992), "Endwall Losses and FLow Unsteadiness in a Turbine Blade Cascade," ASME Journal of Turbomachinery, 114, pp. 191-197.

Ainley, D. G. and Mathieson, G. C. R. (1951), "A Method of Performance Estimation for Axial Flow Turbine," British ARC, R\&M, 2974.

Aunapuu, N. V., Volino, R. J. and K. A. Flack, R. M. S. (2000), "Secondary Flow Measurements in a Turbine Passage With Endwall Flow Modification," ASME Journal of Turbomachinery, 122, pp. 651-658.

Becz, S., Majewski, M. S. and Langston, L. S. (2003), "Leading Edge Modification Effects on Turbine Cascade Endwall Loss," ASME paper No. GT-2003-38898, Atlanta, GA.

Becz, S., Majewski, M. S. and Langston, L. S. (2004), "An Experimental Investigation of Contoured Leading Edges for Secondary Flow Loss Reduction," ASME paper No. GT-2004-53964, Vienna, Austria.

Benner, M. W. (2003), "The Effects of Leading Edge Geometry on Profile and Secondary Losses in Turbine Cascades," Ph.D. thesis, Carleton University, Ottawa, Canada. 
Benner, M. W., Sjolander, S. A. and Moustapha, S. H. (1997), "Influence of Leading Edge Geometry on Profile Losses in Turbines at Off-Design Incidence: Experimental Results and an Improved Correlation," 116, pp. 193-200.

Benner, M. W., Sjolander, S. A. and Moustapha, S. H. (2004a), "The Influence of Leading Edge Geometry on Secondary Losses in a Turbine Cascades at the Design Incidence," ASME Journal of Turbomachinery, 126, pp. 277-287.

Benner, M. W., Sjolander, S. A. and Moustapha, S. H. (2004b), "Measurements of Secondary Flows Downstream of a Turbine Cascade at Off-Design Incidence," ASME paper No. GT-2004-53786, Vienna, Austria.

Benner, M. W., Sjolander, S. A. and Moustapha, S. H. (2005a), "An Empirical Prediction Method for Secondary Losses in Turbines: Part I - A New Loss Breakdown Scheme and Penetration Depth Correlation," ASME paper No. GT2005-68637, Reno, USA.

Benner, M. W., Sjolander, S. A. and Moustapha, S. H. (2005b), "An Empirical Prediction Method for Secondary Losses in Turbines: Part II - A New Secondary Loss Correlation," ASME paper No. GT-2005-68639, Reno, USA.

Brear, M. J., Hodson, H. P., Gonzalez, P. and Harvey, N. W. (2002), "Pressure Surface Separations in Low-Pressure Turbines - Part 2: Interactions with Secondary Flow," ASME Journal of Turbomachinery, 124, pp. 402-409.

Came, P. (1973), "Secondary Loss Measurements in a Cascade of Turbine Blades," The Institution of Mechanical Engineering, C33/7312, pp. 75-83.

Chen, L. D. and Dixon, S. L. (1986), "Growth of Secondary Flow Losses Downstream of a Turbine Blade Cascade," ASME Journal of Engineering for Gas Turbines and Power, 108, pp. 270-276. 
Dai, W. (2004), "Steady Aerodynamic Performance of a Very Highly Loaded LowPressure Turbine Airfoil," Master's thesis, Carleton University, Ottawa, Canada.

de la Rosa Blanco, E., Hodson, H. P., Vazquez, R. and Torre, D. (2003), "Influence of the State of the Inlet Endwall Boundary Layer on the Interaction between Pressure Surface Spearation and Endwall Flows," Proc. Instn. Mech. Engrs, J. Power and Energy, 217 Part A, pp. 433-441.

DeCecco, S. (1995), "Behaviour of Tip-Leakage Flows at Large Clearances," Master's thesis, Carleton University, Ottawa, Canada.

Denton, J. (1993), "Loss Mechanisms in Turbomachines," ASME Journal of Turbomachinery, 115, pp. 621-656.

Dossena, V., D'Ippolito, G. and Pesatori, E. (2004), "Stagger Angle and Pitch-Chord Ratio Effects on Secondary Flows Downstream of a Turbine Cascade at Several Off-Design Incidences," ASME paper No. GT-2004-54083, Reno, USA.

Dossena, V., Perdichizzi, A. and Savini, M. (1999), "The Influence of Endwall Contouring on the Performance of a Turbine Nozzle Guide Vane," ASME Journal of Turbomachinery, 121, pp. 200-208.

Duden, A. and Fottner, L. (1999), "The Secondary Flow Field of a Turbine Cascade with 3D Airfoil Design and Endwall Contouring at Off-Design Incidence," ASME paper No. GT-1999-211.

Duden, A., Raab, I. and Fottner, L. (1999), "Controlling the Secondary Flow in a Turbine Cascade by Three-Dimensional Airfoil Design and Endwall Contouring," ASME Journal of Turbomachinery, 121, pp. 191-199. 
Dunham, J. (1970), "A Review of Cascade Dataon Secondary Losses in Turbines," Journal of Mechanical Engineering Science, 12, pp. 48-59.

Dunham, J. and Came, P. (1970), "Improvments to the Ainley-Mathieson Method of Turbine Performance Prediction," Journal of Engineering for Power, pp. 252256.

Gallington, R. W. (1980), "Measurement of Very Large Flow Angles with Non-Nulling Seven-Hole Probes," USAFA paper No. TR-80-17.

Gerner, A. A. and Maurer, C. L. (1981), "Calibration of Seven-Hole Probes Suitable for High Angles in Subsonic Compressible Flows," USAFA paper No. TR-81-4.

Goobie, S. (1989), "A Test Section for Investigating the On-Design Performance of Planar Cascades,," Ph.D. thesis, Carleton University, Ottawa, Canada.

Gregory-Smith, D. G. and Cleak, J. G. E. (1992), "Secondary Measurements in a Turbine Cascade With High Inlet Turbulence," ASME Journal of Turbomachinery, 114, pp. 173-183.

Gregory-Smith, D. G., Graves, C. P. and Walsh, J. A. (1988), "Growth of Secondary Losses and Vorticity in aa Axial Turbine Cascade," ASME Journal of Turbomachinery, 110, pp. 1-8.

Gregory-Smith, D. G., Ingram, G., Jayaraman, P., N.Harvey and Rose, M. G. (2001), "Non-Axysymmetric Turbine end Wall Profiling," Proc. Insitution of Mechanical Engineers, 215, pp. 721-734.

Gregory-Smith, D. G., Walsh, J. A., Graves, C. P. and Fulton, K. (1988), "Turbulence Measurements and Secondary Flows in a Turbine Rotor Cascade," ASME Journal of Turbomachinery, 110, pp. 479-485. 
Greitzer, E., Tan, C. and M.B.Graf (2004), Internal Flow: Concepts and Applications, 1st ed., Cambridge University Press, Cambridge.

Harrison, S. (1990), "Secondary Loss Generation in a Linear Cascade of High-Turning Turbine Blades," ASME Journal of Turbomachinery, 112, pp. 618-624.

Harvey, N. W., Brennan, G., Newman, D. A. and Rose, M. G. (1999), "Improving Turbine Efficiency Using Non-Axysymmetric End Walls: Validation in the MultiRow Environment and With Low Aspect Ratio Blading," ASME paper No. GT1999-211.

Hawthorne, W. R. (1955), "Rotational Flow Through Cascades Part 1 - The Components of Vorticity," Journal of Mech. and Applied Math., 3.

Ho, Y. and Lakshminarayana, B. (1996), "Computational Modeling of Three Dimensional Endwall Flow Through a Turbine Rotor With Strong Secondary Flows," ASME Journal of Turbomachinery, 118, pp. 250261.

Hodson, H. P. (1984), "Boundary Layer and Loss Measurements on the Rotor of an Axial-Flow Turbine," ASME Journal of Engineering for Power, 106, pp. 391399.

Hodson, H. P. (1998b), "Bladerow Interactions In Low Pressure Turbines," Blade Row Interference Effects in Axial Turbomachinery Stages, edited by H. P. Hodson and C. H. Sieverding, VKI Lecture Series 1998-02, Von Karman Institute, Belgium.

Hodson, H. P. and Dominy, R. G. (1987a), "Boundary Layer and Loss Measurements on the Rotor of an Axial-Flow Turbine," ASME Journal of Turbomachinery, 109, pp. 391-399. 
Hodson, H. P. and Dominy, R. G. (1987b), "The Off-Design Performance of a LowPressure Turbine Cascade," ASME Journal of Turbomachinery, 109, pp. 201209.

Howell, R. J., Ramesh, O. N., Hodson, H. P., Harvey, N. V. and Schulte, V. (2001), "HighLiftandAft-LoadedProfilesforLow-PressureTurbines," ASME Journal of Turbomachinery, 123, pp. 181-188.

Ingram, G., Gregory-Smith, D. and N.Harvey (2005), "Investigation of a Novel Secondary Flow Feature in a Turbine Cascade With End Wall Profiling," ASME Journal of Turbomachinery, 127, pp. 209-214.

Ingram, G., Gregory-Smith, D., Rose, D., N.Harvey and Brennan, G. (2002), "The Effect of End-Wall Profiling on Secondary Flow and Loss Development in a Turbine Cascade," ASME paper No. GT-2002-30339.

Islam, A. M. T. and Sjolander, S. A. (1999), "Deviation in Axial Turbines at Subsonic Conditions," ASME paper No. 99-GT-026, Indianapolis, IN.

Jeffries, M. S. (2001), "Initial Investigations of Transonic Turbine Aerodynamics Using the Carleton University High-Speed Wind Tunnel," Ph.D. thesis, Carleton University, Ottawa, Canada.

Kacker, S. and Okapuu, U. (1982), "A Mean Line Prediction Method for Axial Flow Turbines Efficiency," Journal of Engineering for Power, 104, pp. 111-119.

Kawai, T. (2005), "Effect of Combined Boundary Layer Fences on Turbine Secondary Flow and Losses," ASME Journal of Turbomachinery, 127, pp. 209-214.

Langston, L. S. (1989), "Research on Cascade Secondary and Tip-Leakage Flows Periodicity and Surface Flow Visualisation," CP-469, pp. 1-13. 
Langston, L. S. (2001), "Secondary Flows in Axial Turbines - A Review," Annals of the New York Academy of Sciences, 934, pp. 11-26.

Langston, L. S., Nice, M. L. and Hooper, R. M. (1977), "Three-Dimensional Flow Within a Turbine Cascade Passage," Journal of Engineering for Power, pp. 2128.

Lewis, A. B. (1966), "Fixed-Direction Probes for Aerodynamic Measurements," Proc. Inst. Mech. Engrs, Pt.3J, 180, pp. 141-152.

Lord, W. K., MacMartin, D. G. and Tillman, T. G. (2000), "Flow Control Opportunities in Gas Turbines," AIAA paper No. No.2000-2234, Denver,CO.

Mahallati, A. (2003), "Aerodynamics of a Low-Pressure Turbine Airfoil Under Steady and Periodically Unsteady Conditions," Ph.D. thesis, Carleton University, Ottawa, Canada.

Marchal, P. (1980), "Etude des ecoulements secondaires en grille d'aubes de detente," Ph.D. thesis, Universitet Liobre de Bruxelles, Brussels, Belgium.

McAuliffe, B. (2003), "An Experimental Study of Flow Control Using Blowing for a Low-Pressure Turbine Airfoil," Master's thesis, Carleton University, Ottawa, Canada.

Moffat, R. J. (1982), "Contributions to the Theory of Single-Sample Uncertainty," Journal of Fluid Engineering, 104, pp. 250-260.

Moore, J. and Adhye, R. (1985), "Secondary Flows and Losses Downstream of a Turbine," ASME Journal of Turbomachinery, 107, pp. 961-968.

Moore, J. and Moore, J. G. (1985), "Performance evaluation of linear turbine cascade using three-dimensional viscous flow calculations," ASME paper No. 85-GT-65. 
Moustapha, S. H., Kacker, S. C. and Tremblay, B. (1990), "An Improved Incidence Losses Prediction Mehod for Turbine Airfoils," ASME Journal of Turbomachinery, 112, pp. 267-276.

Moustapha, S. H., Paron, G. and Blade, J. (1985), "Secondary Flows in Cascades of Highly Loaded Turbine Blades," Journal of Engineering for Gas, 107, pp. 10311038.

Perdichizzi, A. (1990), "Mach Number Effects on Secondary Flow Development Downstream of a Turbine Cascade," ASME Journal of Turbomachinery, 112, pp. 643651 .

Perdichizzi, A. and Dossena, V. (1993), "Incidence Angle and Pitch-Chord Effects on Secondary Flows Downstream of a Turbine Cascade," ASME Journal of Turbomachinery, 115, pp. 383-391.

Popovic, I. (2005), "The Effects of Leading Edge Geometry on Profile and Secondary Losses in Turbine Cascades," Master's thesis, Carleton University, Ottawa, Canada.

Rodger, P. W. (1992), "Establishing Two-Dimensional Flow in a Large Scale Planar Turbine Cascade," Master's thesis, Carleton University, Ottawa, Canada.

Sauer, H., Mueller, R. and Vogeler, K. (2001), "Reduction of Secondary Flow Losses in Turbine Cascades by Leading Edge Modification at the Endwall," ASME Journal of Turbomachinery, 123, pp. 207-213.

Sauer, H. and Wolf, H. (1994), "The Influence of the Inlet Boundary Layers on the Secondary Losses of Turbine Stages," Technology Requirements for Small Gas Turbines No. AGARD-CP-537, paper 26. 
Schlichting, H. and Gersten, K. (2000), Boundary-Layer Theory, 8th ed., SpringerVerlag, Berlin.

Sharma, O. P. and Butler, T. L. (1987), "Predictions of Endwall Losses and Secondary Flows in Axial Turbine Cascades," ASME Journal of Turbomachinery, 109, pp. 229-236.

Shih, T. I. P. and Lin, Y. L. (2002), "Controlling Secondary-Flow Structure by Leading-Edge Airfoil Fillet and Inlet Swirl to Reduce Aerodynamic Loss and Surface Heat Transfer," ASME paper No. GT-2002-30529.

Sieverding, C. H. (1985), "Recent Progress in the Understanding of Basic Aspects of Secondary FLows in Turbine Blade Passages," Journal of Engineering for Gas Turbines and Power, 107, pp. 248-257.

Sieverding, C. H. and den Bosche, P. V. (1983), "The use of Colored Smoke to Visualize Secondary Flows in a Turbine-Blade Cascade," Journal of Fluid Mechanics, 134, pp. 85-89.

Sieverding, C., VanHove, W. and Boletis, E. (1984), "Experimental Study of the Three-Dimensional Flow Field in an Annular Turbine Nozzle Guidevane," Journal of Engineering for Gas Turbines and Power, 106, pp. 437-444.

Walsh, J. and Gregory-Smith, D. G. (1990), "Inlet Skew and the Growth of Secondary Losses and Vorticity in a Turbine Cascade," ASME Journal of Turbomachinery, 112, pp. 633642

Wang, H. P., Olson, S. J., Goldstein, R. J. and Eckert, E. (1997), "Flow Visualization in Linear Turbine Cascade of High Performance Turbine Blades," ASME Journal of Turbomachinery, 119, pp. 1-8. 
Weiss, A. P. and Fottner, L. (1995), "The Influence of Load Distribution on Secondary Flow in Straight Turbine Cascades," ASME Journal of Turbomachinery, 117, pp. $133-141$.

Yamamoto, A. (1987), "Production and Development of Secondary Flows and Losses in Two Types of Straight Turbine Cascades: Part 1-AStator Case," ASME Journal of Turbomachinery, 109, pp. 186-193.

Yamamoto, A. and Nouse, H. (1988), "Effects of Incidence on Three-Dimensional Flows in a Linear Turbine Cascade," ASME Journal of Turbomachinery, 110, pp. 486-496.

Zhang, X. F. (2002), "Hot Film Measurement of the Steady and Unsteady Boundary Layer Development on a Low-Pressure Turbine Airfoil," Master's thesis, Carleton University, Ottawa, Canada. 


\section{Appendix A}

\section{Calibration Coefficients for}

\section{Pressure Transducers}

This appendix explains the procedure used to obtain the calibration coefficients for pressure transducers. As discussed in Section 3.5.1, the output voltage, E, of the transducers exhibits a linear relationship with the measured pressure differential, $\Delta P$, as given by:

$$
\Delta P=C_{1} E+C_{2}
$$

The slope, $C_{1}$, was determined through the calibration of pressure transducers using water micromanometer designed and described into greater detail by Benner (2003). During calibrations at least 50 points were taken over the full range of transducers operation, which for the present measurements was typically up to $\pm 500 \mathrm{~Pa}$. The pressure difference versus output voltage obtained through one of the calibrations is shown in Figure A.1. The transducers were calibrated once per month for the duration of the experiment. On the other hand, the zero offset, $C_{2}$, was recorded 


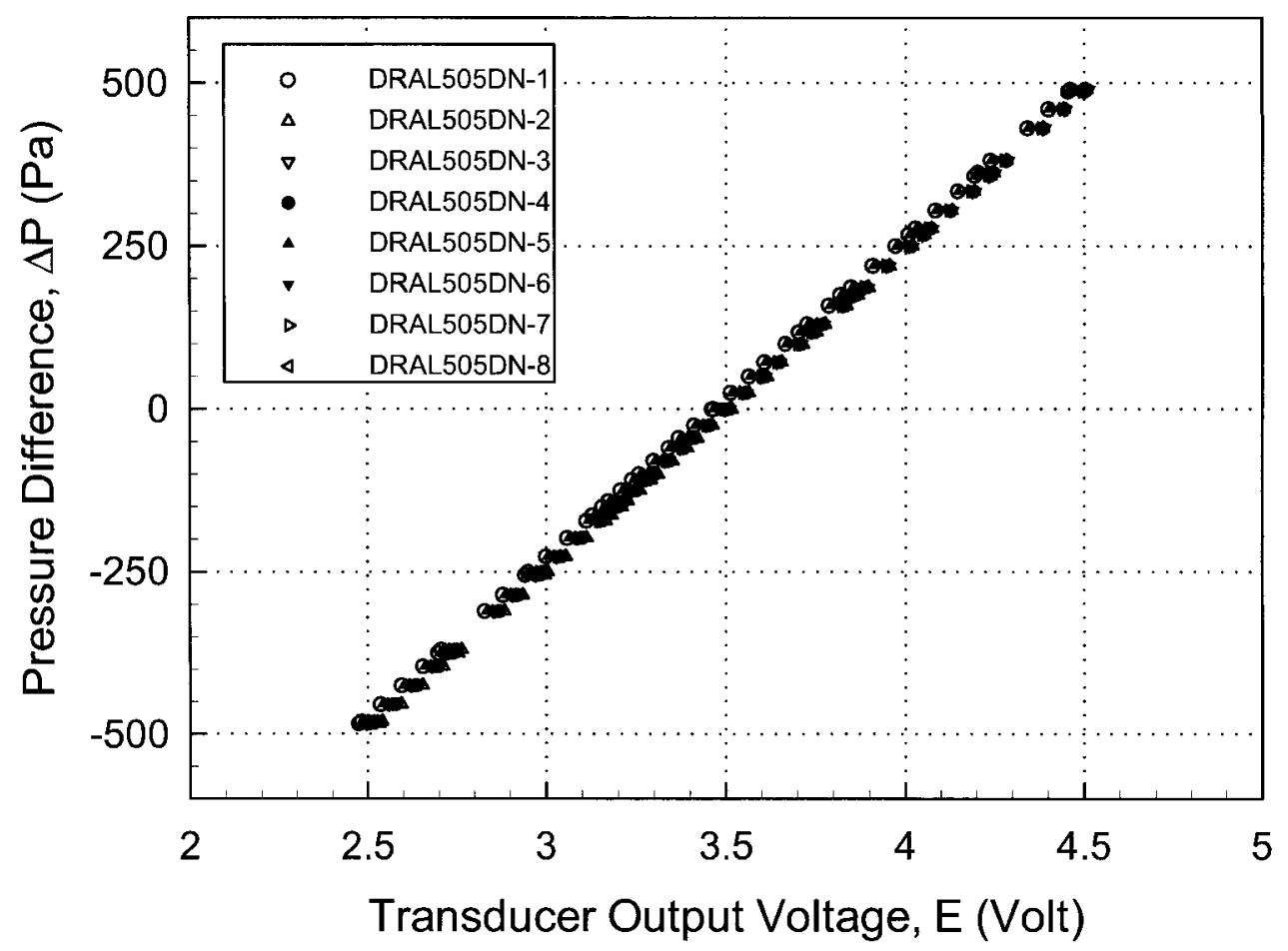

Figure A.1: Sample Calibration Curves

prior and after each experiment.

A typical set of calibration coefficients for all transducers is shown in Table A.1.

Table A.1: Pressure Transducers Calibration Coefficients

\begin{tabular}{|l|c|c|}
\hline Type & $\mathbf{C}_{1}$ & $\mathbf{C}_{\mathbf{2}}$ \\
\hline DRAL505DN-1 & 489.8528 & -1695.6993 \\
\hline DRAL505DN-2 & 493.7192 & -1734.4516 \\
\hline DRAL505DN-3 & 484.9903 & -1695.1677 \\
\hline DRAL505DN-4 & 489.4090 & -1715.8674 \\
\hline DRAL505DN-5 & 490.9272 & -1701.5952 \\
\hline DRAL505DN-6 & 487.5275 & -1711.4867 \\
\hline DRAL505DN-7 & 487.3252 & -1703.8506 \\
\hline DRAL505DN-8 & 486.2682 & -1698.8749 \\
\hline
\end{tabular}

\begin{tabular}{|l|c|c|}
\hline Type & $\mathbf{C}_{\mathbf{1}}$ & $\mathbf{C}_{\mathbf{2}}$ \\
\hline DRAL501DN-1 & 98.9495 & -348.6520 \\
\hline DRAL501DN-2 & 96.2803 & -333.9340 \\
\hline DRAL501DN-3 & 96.3863 & -333.5172 \\
\hline DRAL501DN-4 & 96.0687 & -333.3512 \\
\hline 113LU20D-PCB-1 & 2014.9740 & -7031.5440 \\
\hline 113LU20D-PCB-2 & 2013.8100 & -7037.5240 \\
\hline
\end{tabular}




\section{Appendix B}

\section{Three-Hole Probe Calibration}

\section{Curves}

The calibration rig, described in Section 3.5.5, was used to calibrate the threehole probe using the method proposed by Lewis (1966). After the probe was aerodynamically aligned with the flow, the probe was rotated for $\pm 20^{\circ}$ in increments of $0.5^{\circ}$. The calibration file that is used by CarletonDaq to process the raw three-hole probe data consists of a look-up table with six coefficients for each angle. Calibration coefficients were calculated from the following equations as given by Lewis (1966):

$$
\begin{aligned}
& K_{1}=\frac{P_{1}-P_{S}}{P_{0}-P_{S}}=C_{p 1} \\
& K_{12}=\frac{P_{1}-P_{2}}{P_{0}-P_{S}}=C_{p 1}-C_{p 2} \\
& K_{13}=\frac{P_{1}-P_{3}}{P_{0}-P_{S}}=C_{p 1}-C_{p 3}
\end{aligned}
$$




$$
\begin{aligned}
& K_{23}=\frac{P_{2}-P_{3}}{P_{0}-P_{S}}=C_{p 2}-C_{p 3} \\
& K_{\phi 2}=\frac{P_{1}-P_{2}}{P_{0}-P_{S}}=\frac{K_{12}}{K_{13}} \\
& K_{\phi 3}=\frac{P_{1}-P_{2}}{P_{0}-P_{S}}=\frac{1}{K_{\phi 2}}
\end{aligned}
$$

Two different calibration sets were used for the inlet and outlet traverses, corresponding to the mean velocities at the respective planes. Typical calibration plots are shown in Figures B.1 and B.2. More details on the calibration procedure and the effects of the Reynolds number on the calibration data are given by Mahallati (2003). 

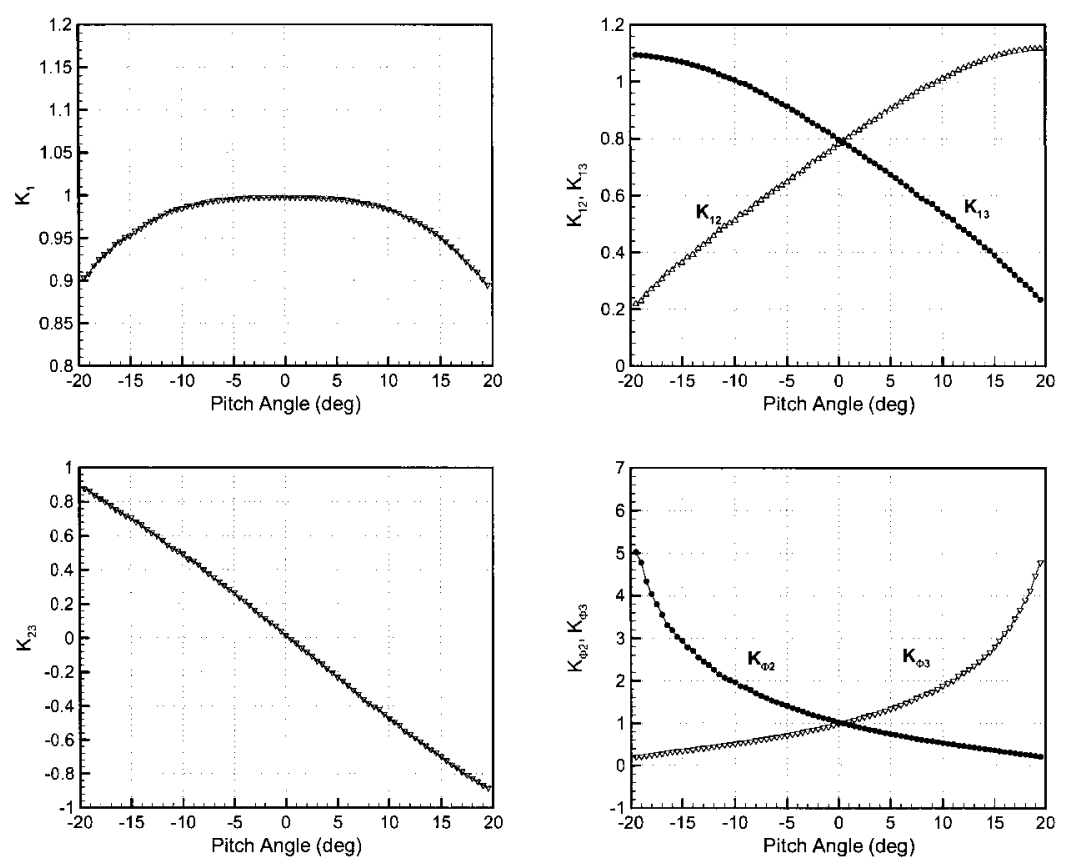

Figure B.1: Three-Hole Probe Calibration Curves

(Cascade Inlet Conditions: $V_{1} \approx 15.5 \mathrm{~m} / \mathrm{s}$ )
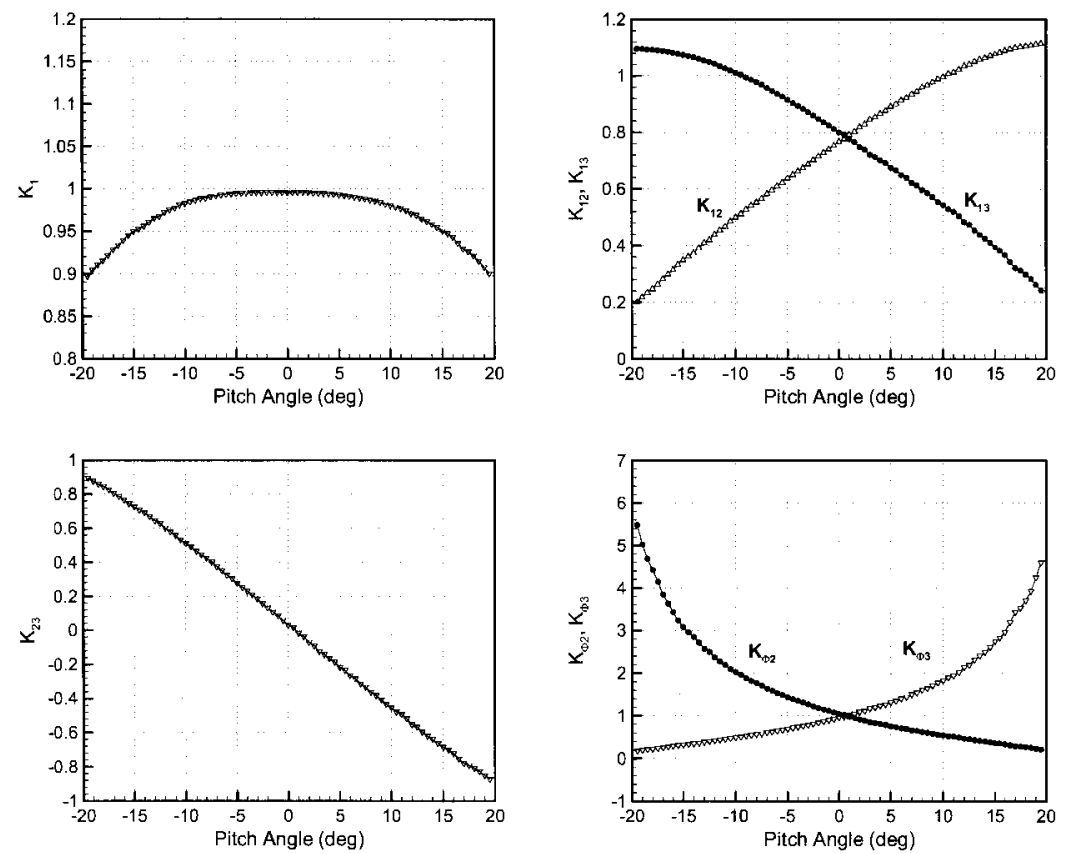

Figure B.2: Three-Hole Probe Calibration Curves

(Cascade Outlet Conditions: $V_{2} \approx 24.0 \mathrm{~m} / \mathrm{s}$ ) 


\section{Appendix C}

\section{Seven-Hole Probe Calibration}

\section{Curves}

This appendix provides a brief description of the seven-hole calibration data sets and calibration curves. The method for seven-hole probe calibration and data reduction was developed by Gallington (1980) (referred to by Gerner and Maurer (1981). It is valid only for incompressible flow conditions. The same probe was used and calibrated by both DeCecco (1995) and Benner (2003) who provided more details on the calibration procedure.

The calibration was performed separately for low- and high-angle flow regimes. The low-angle flow regime is defined as the condition at which the central hole (port) has the highest pressure reading. This was typically the case for the flow angles of $25^{\circ}$ or less. After the probe was aerodynamically aligned with the flow in the calibration rig, both $\alpha$ and $\beta$, as defined in Figure C.1, were varied over the range of $\pm 25^{\circ}$ in increments of $5^{\circ}$. A calibration file consists of the following four calibration 
coefficients for each flow angle pair $(\alpha, \beta)$ :

$$
\begin{gathered}
C_{\alpha}=\frac{2 C_{\alpha_{1}}+C_{\alpha_{2}}-C_{\alpha_{3}}}{3} \\
C_{\beta}=\frac{C_{\alpha_{2}}+C_{\alpha_{3}}}{\sqrt{ } 3} \\
C_{0}=\frac{P_{7}-P_{0}}{P_{7}-\bar{P}_{1-6}} \\
C_{q}=\frac{P_{7}-\bar{P}_{1-6}}{P_{0}-P}
\end{gathered}
$$

where $P_{7}$ is the pressure at the middle hole (port 7 as shown in Figure C.2) and $\bar{P}_{1-6}$ is the average of outer six holes which approximate local total and static pressures, respectively. $C_{\alpha_{1}}, C_{\alpha_{2}}$ and $C_{\alpha_{3}}$ can be found from:

$$
\begin{aligned}
C_{\alpha_{1}} & =\frac{P_{4}-P_{1}}{P_{7}-\bar{P}_{1-6}} \\
C_{\alpha_{1}} & =\frac{P_{3}-P_{6}}{P_{7}-\bar{P}_{1-6}} \\
C_{\alpha_{1}} & =\frac{P_{2}-P_{5}}{P_{7}-\bar{P}_{1-6}}
\end{aligned}
$$

Figures from C.3 to C.6 present a typical calibration data for low-angle regime. 


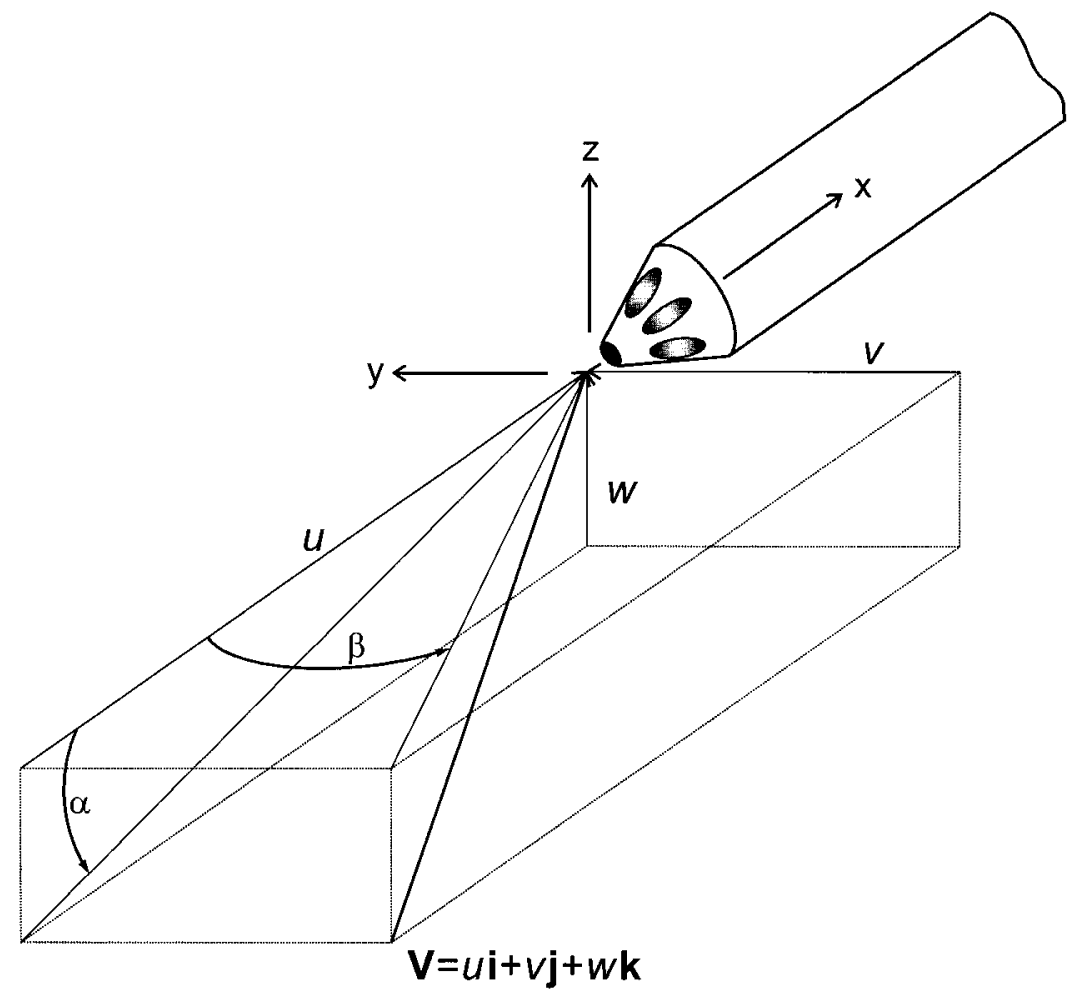

Figure C.1: $C_{\alpha}$ Tangential Coordinate System for Low-Angle Regime (reproduced from DeCecco, 1995)
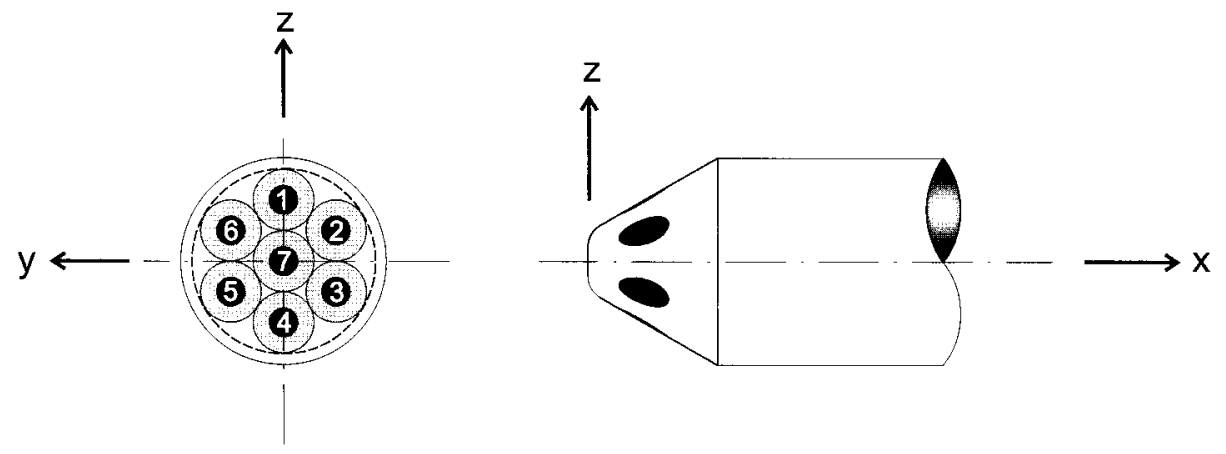

Figure C.2: $C_{\alpha}$ Seven-Hole Probe Numbering Scheme (reproduced from Benner, 2003) 

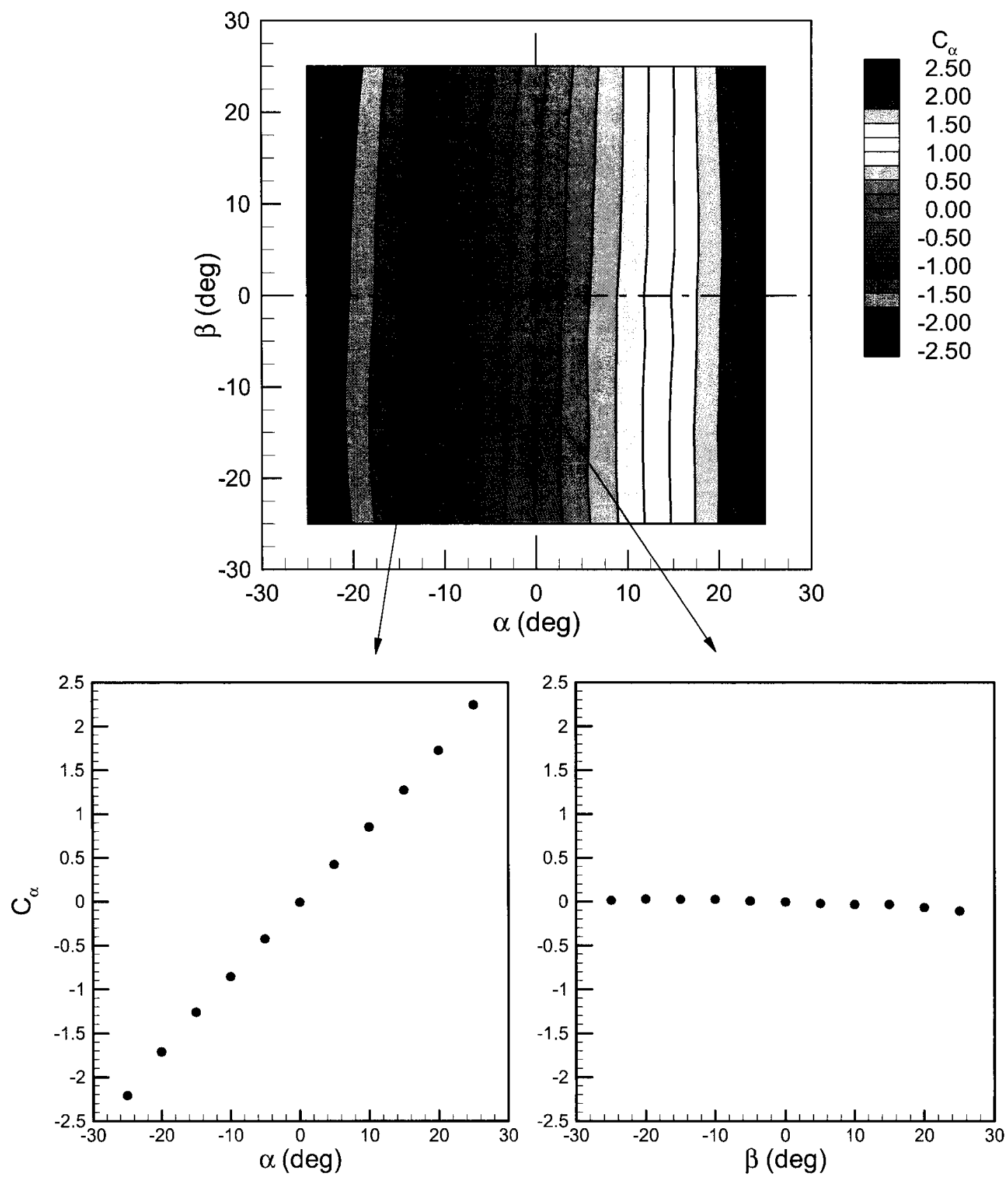

Figure C.3: $C_{\alpha}$ Calibration Data for Sector 7 

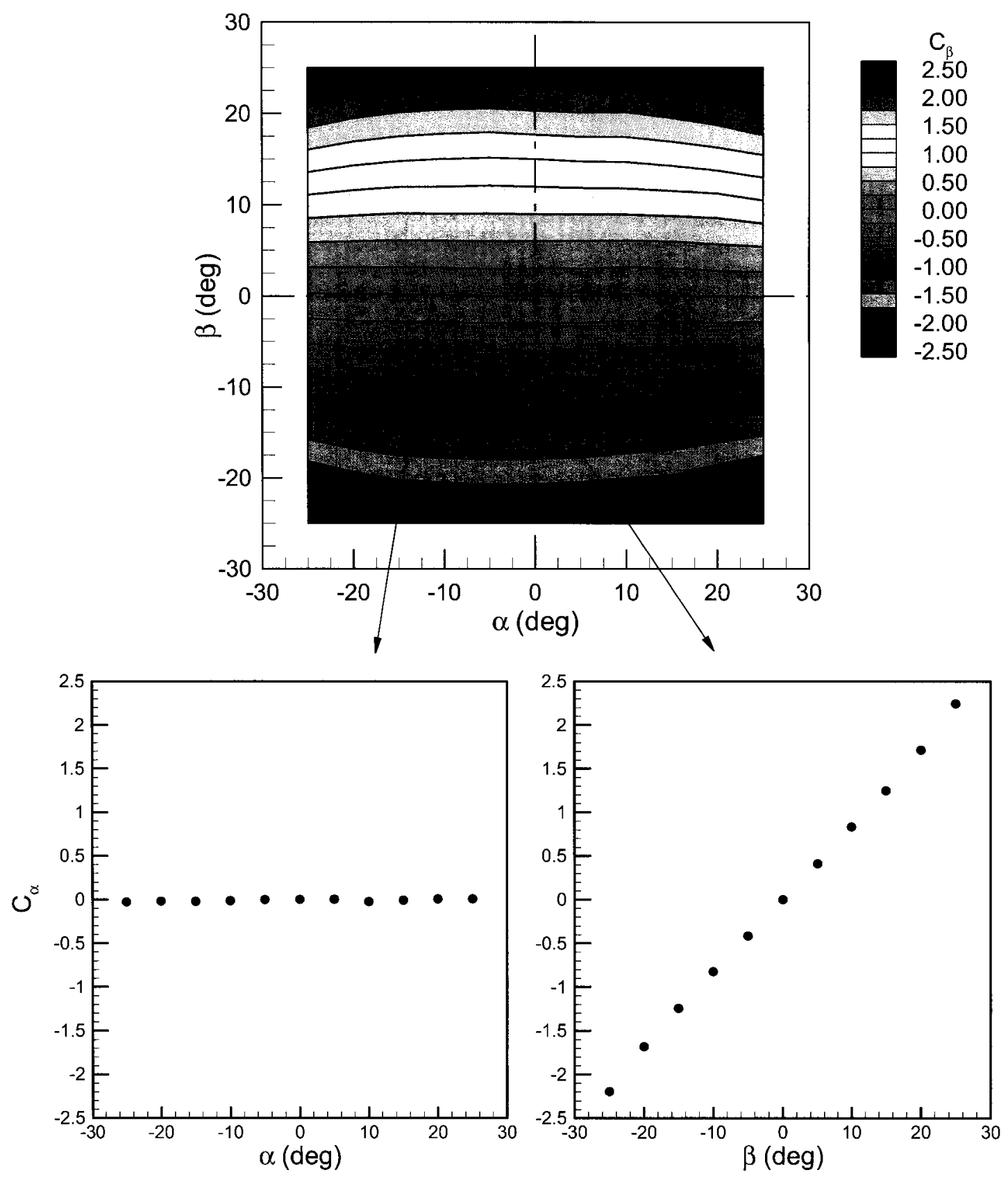

Figure C.4: $C_{\beta}$ Calibration Data for Sector 7 

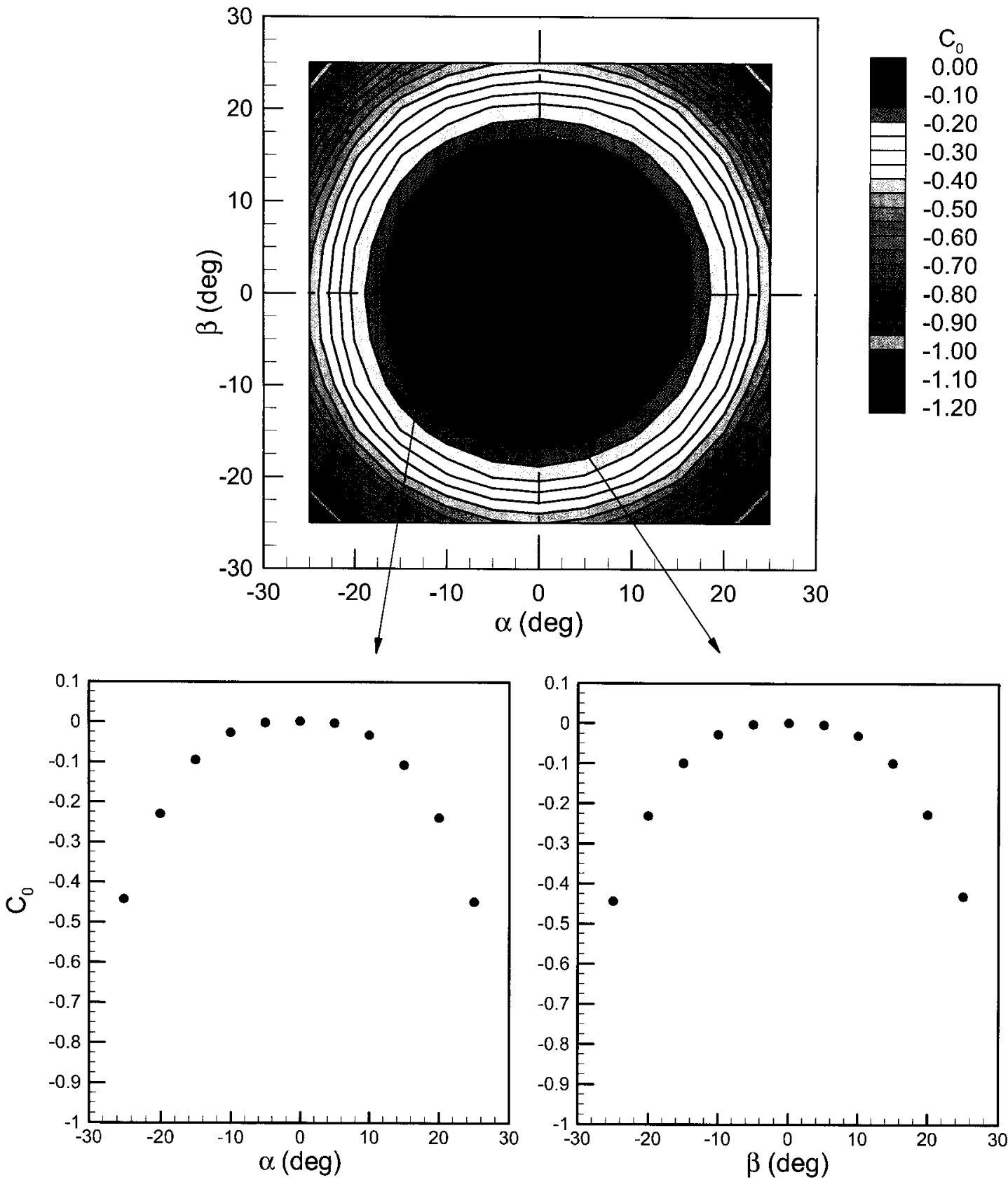

Figure C.5: $C_{0}$ Calibration Data for Sector 7 

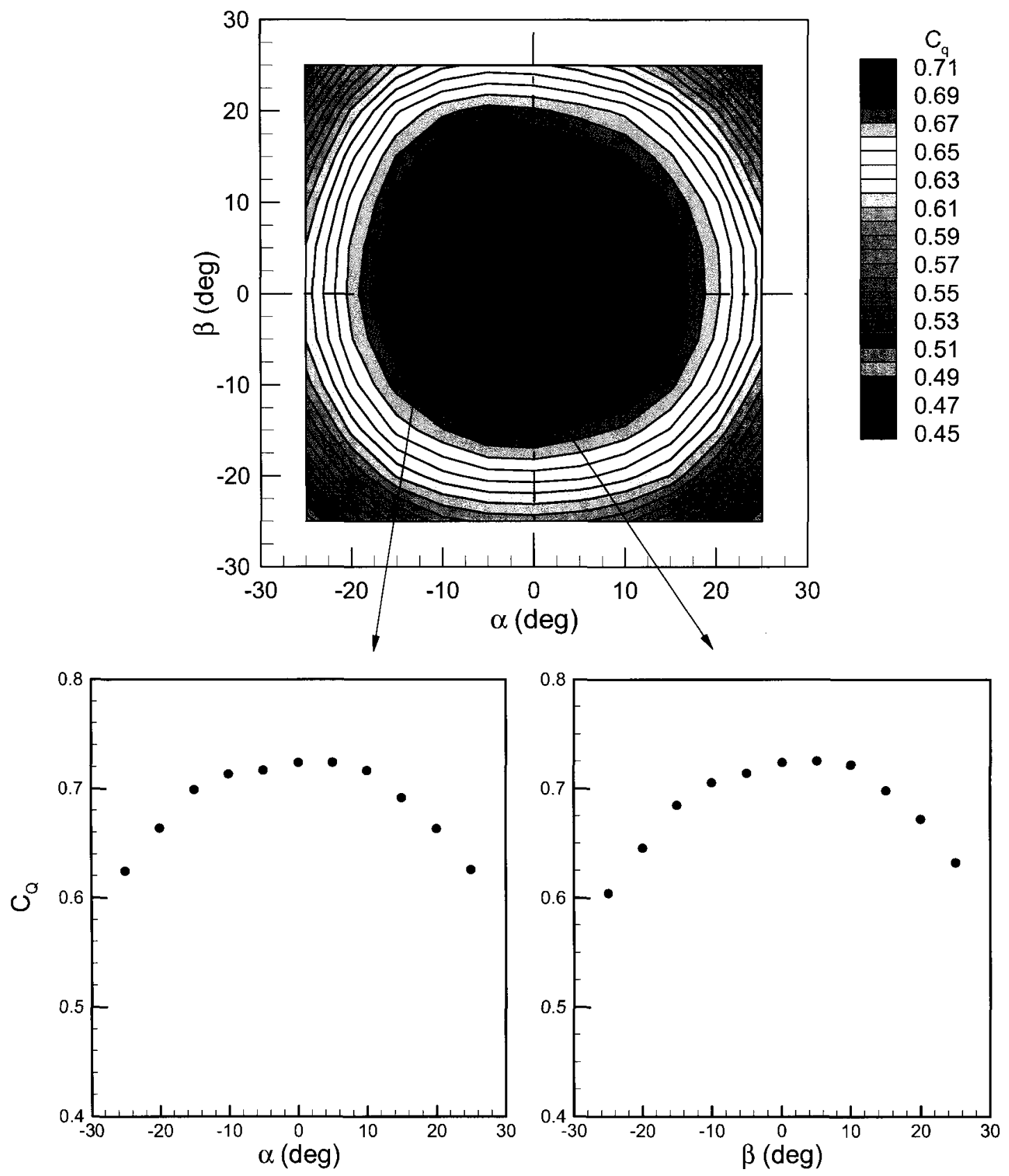

Figure C.6: $C_{Q}$ Calibration Data for Sector 7 
This data set was used for data reduction as long as the middle hole was reading the highest pressure. If one of the surrounding ports was exposed to the highest pressure, one of the corresponding high-angle flow regime calibration sets was used. For highangle regime (flow angles of $25^{\circ}$ or more) a polar coordinate system was defined as shown in Figure C.7. At these conditions, the flow is usually separated over the face of the probe. Thus, only the middle hole, the highest reading hole and the two adjacent hole data were used for the calculation of calibration coefficients. The full calibration set for high-angle regime consists of six separate files each corresponding to a different zone as defined in Figure C.8. Similarly to low-angle dataset, for each of the zones the calibration dataset contains following four calibration coefficients for every flow angle pair $(\theta, \phi)$ :

$$
\begin{aligned}
C_{\theta_{n}} & =\frac{P_{n}-P_{7}}{P_{n}-\left(\frac{P_{n-}+P_{n+}}{2}\right)} \\
C_{\phi_{n}} & =\frac{P_{n-}-P_{n+}}{P_{n}-\left(\frac{P_{n-+}+P_{n+}}{2}\right)} \\
C_{0} & =\frac{P_{n}-P_{0}}{P_{n}-\left(\frac{P_{n-}+P_{n+}}{2}\right)} \\
C_{q} & =\frac{P_{n}-\left(\frac{P_{n-}+P_{n+}}{2}\right)}{P_{0}-P}
\end{aligned}
$$

where the subscript $\mathrm{n}$ corresponds to the highest reading pressure port, while the subscripts $n+$ and $n$ - correspond to the adjacent holes clockwise and counterclockwise relative to port n, respectively. Figures C.9 to C.12 present a typical calibration data for one of the outer zones (zone 4). 


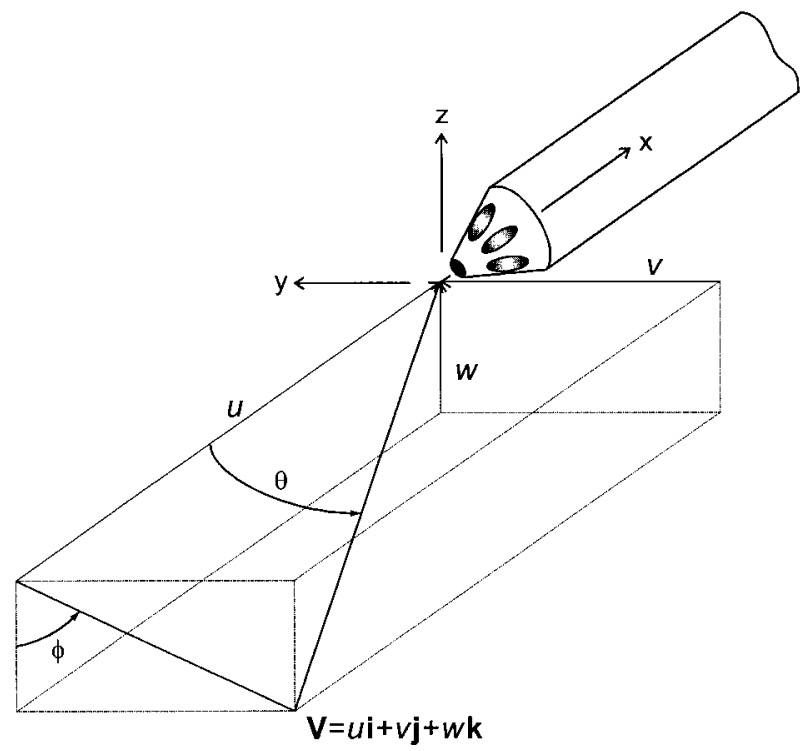

Figure C.7: $C_{\alpha}$ Polar Coordinates System Used for High-Angle Regime (reproduced from DeCecco, 1995)

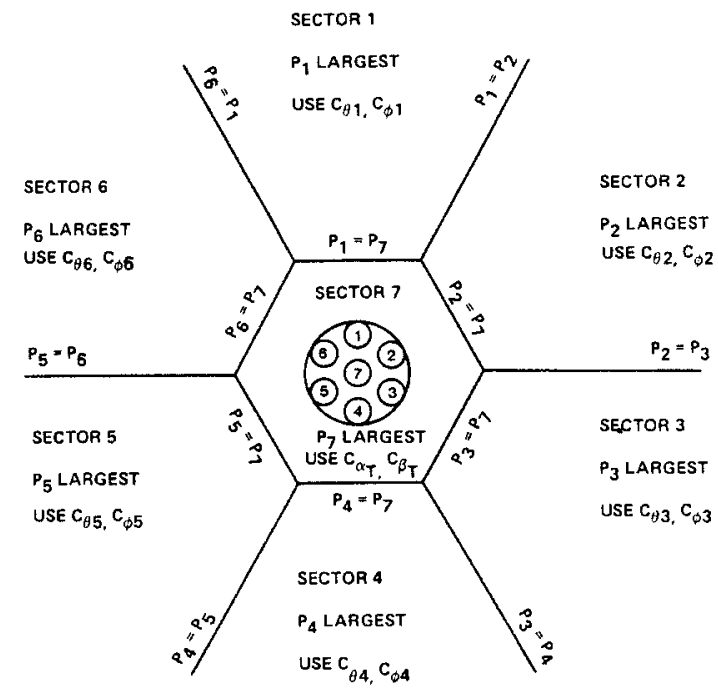

Figure C.8: $C_{\alpha}$ Division of Angular Space

(reproduced from Gerner and Maurer, 1981) 

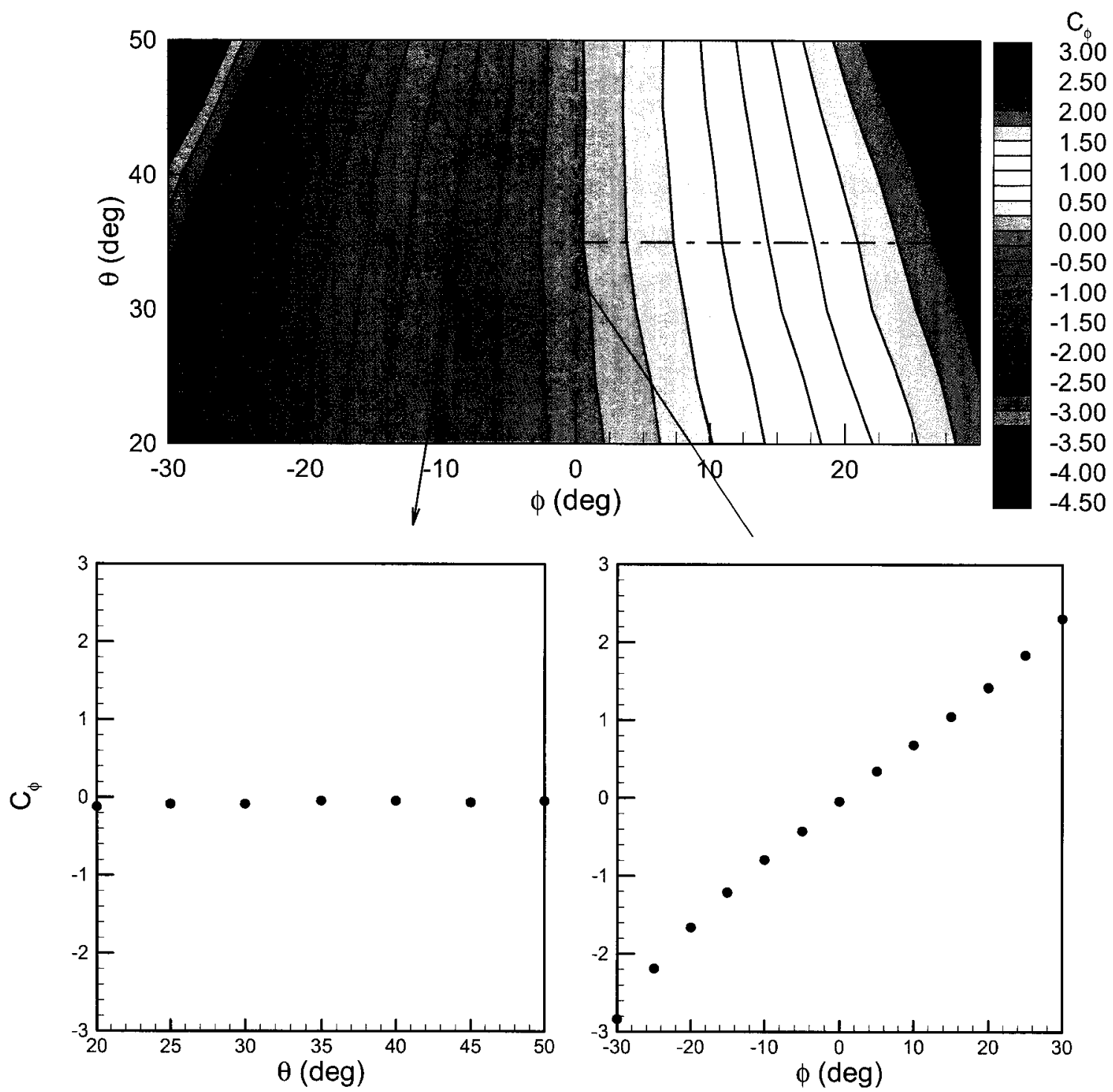

Figure C.9: $C_{\phi}$ Calibration Data for Sector 4 

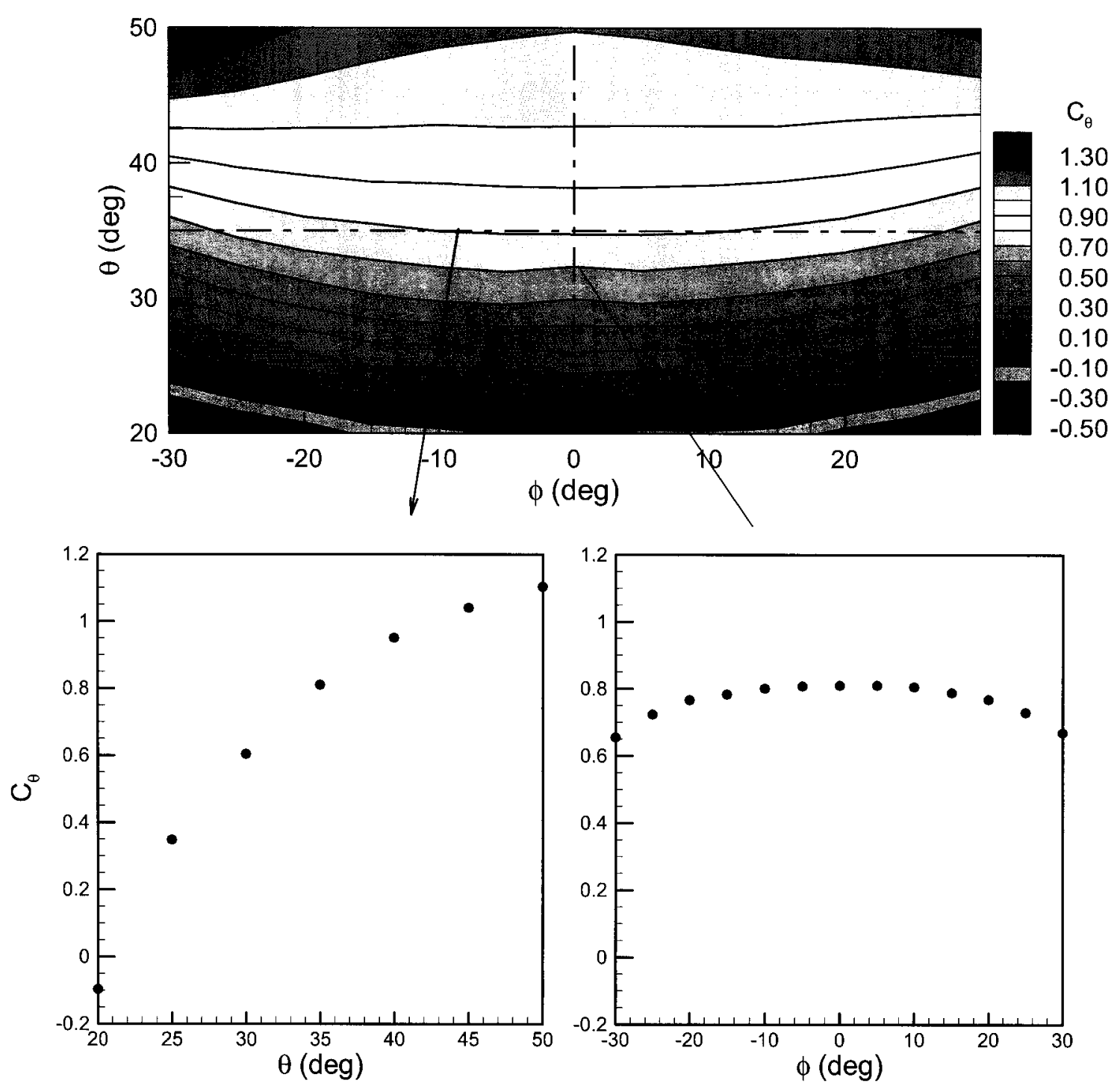

Figure C.10: $C_{\theta}$ Calibration Data for Sector 4 

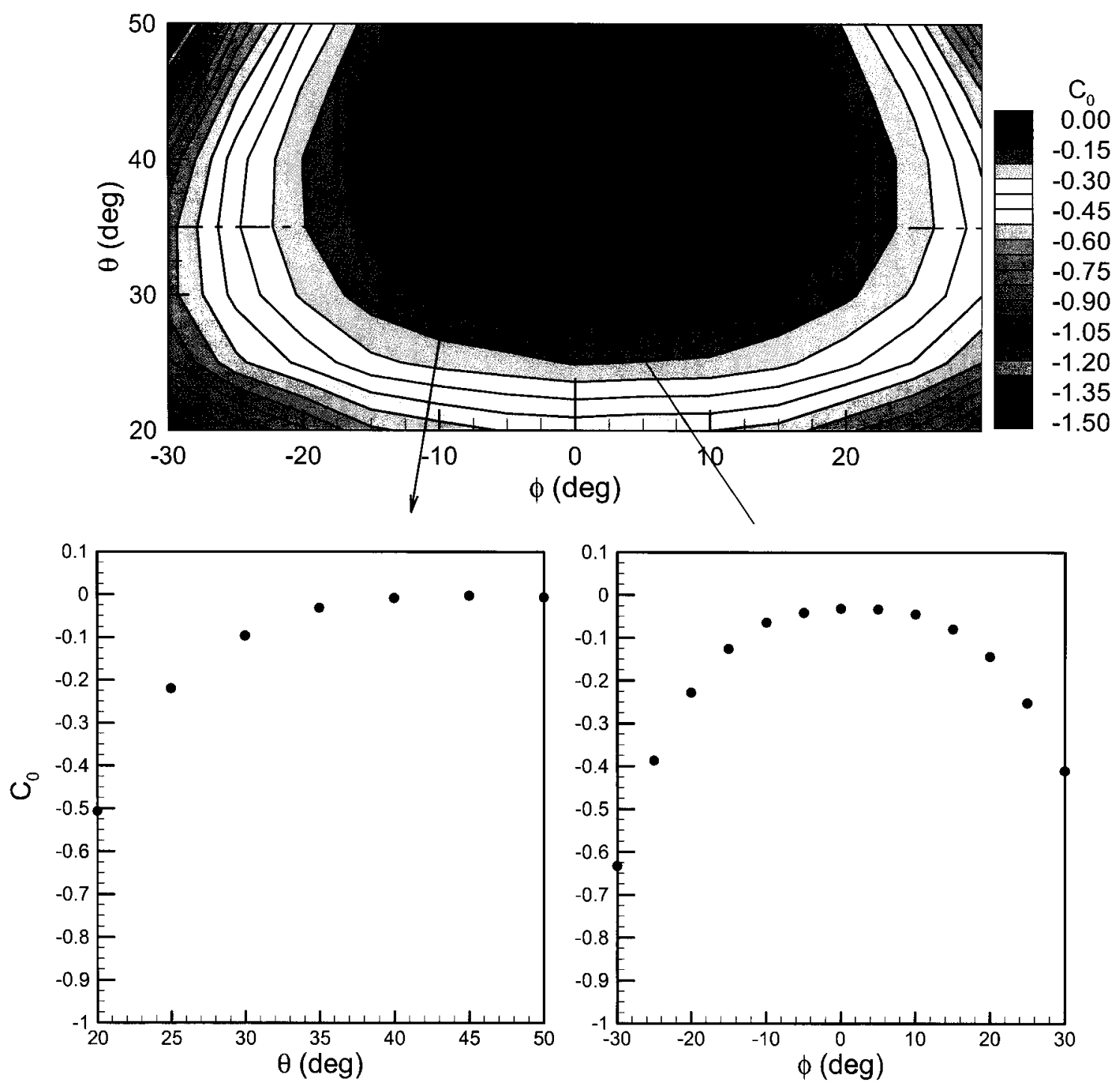

Figure C.11: $C_{0}$ Calibration Data for Sector 4 

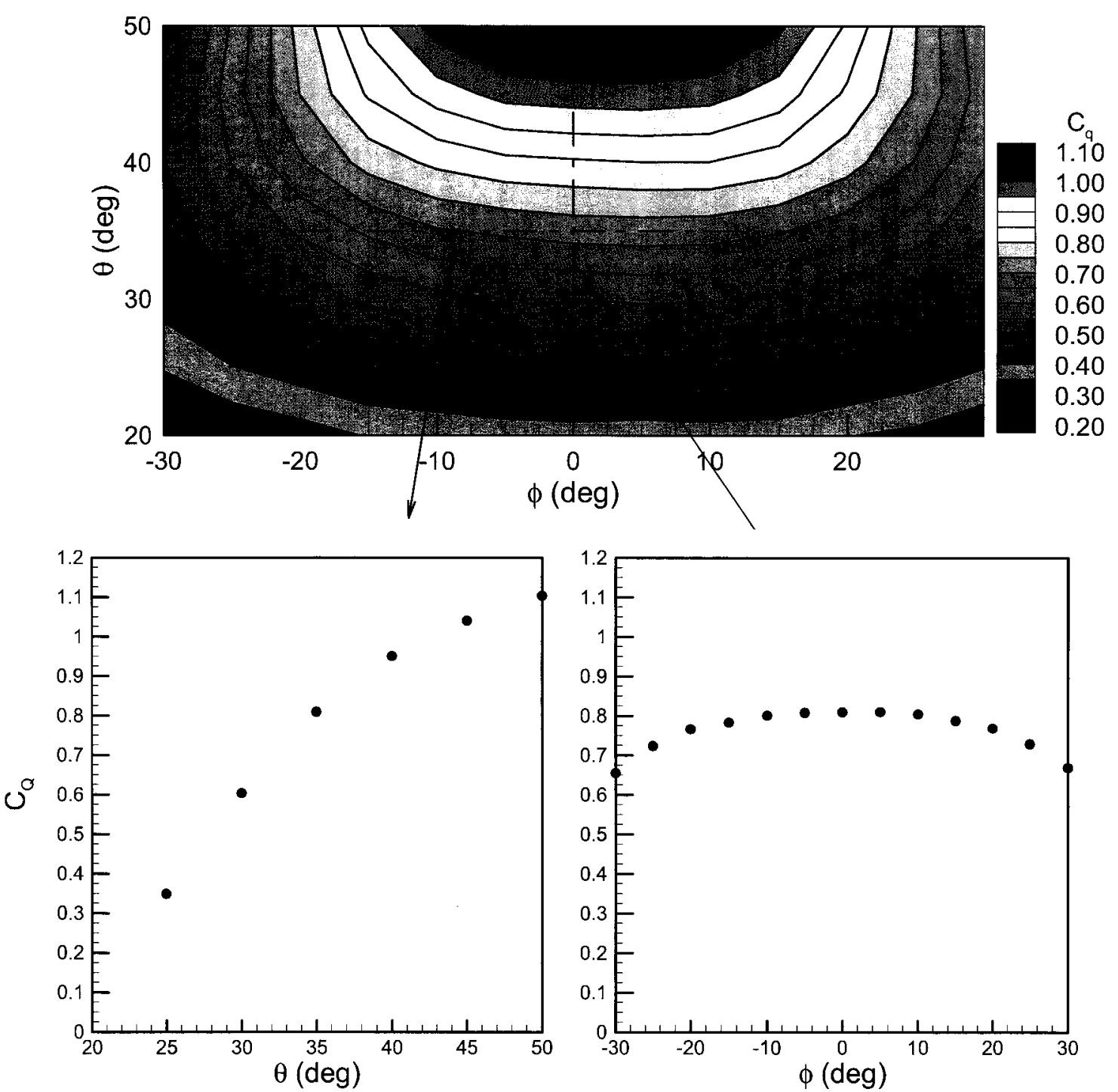

Figure C.12: $C_{Q}$ Calibration Data for Sector 4 


\section{Appendix D}

\section{Summary of Experimental Results at Design Incidence}

This appendix provides the summary of mass-averaged results for all three airfoils at design incidence and two FSTIs. Tables D.1 and D.2 present the result summary at FSTI of $1.5 \%$ and $4.0 \%$, respectively.

The values of profile losses in the measurement and mixed-out planes and AVR at the midspan were provided by Popovic (2005).

As mentioned previously, the superscripts ' and " indicate the pitchwise (for midspan measurements) and pitch and spanwise (for full rectangle) mass-averaged results, respectively.

Subscript 2 means that it refers to outlet plane, CL stands for the centerline and SKE represents the secondary kinetic energy. The remaining subscripts are selfexplanatory. 
Table D.1: Summary of Results at Design Incidence, FSTI $=\mathbf{1 . 5 \%}$

\begin{tabular}{|c|c|c|c|c|}
\hline & & PAKB & PAKD-A & PAKD-F \\
\hline \multirow{13}{*}{ 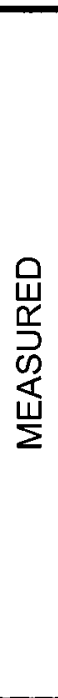 } & 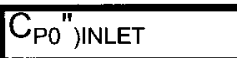 & -0.017 & -0.012 & -0.012 \\
\hline & $\mathrm{C}_{\mathrm{P} 0 \text { "JOUTLET }}$ & -0.145 & -0.186 & -0.160 \\
\hline & $\mathrm{C}_{\mathrm{PO}}{ }^{\prime \prime}$ )TOTAL & -0.128 & -0.174 & -0.148 \\
\hline & $Y_{\text {TOTAL }}$ & 0.056 & 0.080 & 0.064 \\
\hline & $Y_{\text {PROFILE }}$ & 0.034 & 0.057 & 0.034 \\
\hline & $Y_{\text {SECONDARY }}$ & 0.022 & 0.023 & 0.030 \\
\hline & $\mathrm{C}_{\mathrm{T}+}$ & 0.328 & 0.385 & 0.405 \\
\hline & $\mathrm{C}_{\Gamma-}$ & -0.260 & -0.253 & -0.302 \\
\hline & $\mathrm{C}_{\text {SKE }}{ }^{\prime}$ & 0.020 & 0.018 & 0.026 \\
\hline & $\alpha_{2}{ }^{\prime \prime}$ [deg] & -56.4 & -56.5 & -57.9 \\
\hline & $Q_{2} / Q_{C L}{ }^{\prime \prime}$ & 2.28 & 2.18 & 2.31 \\
\hline & AVR' & 0.98 & 1.01 & 0.98 \\
\hline & $Q$ & 4.4 & 5.9 & 6.6 \\
\hline \multirow{9}{*}{ 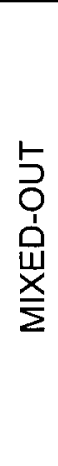 } & $\mathrm{C}_{\mathrm{PO}}{ }^{\prime}$ )OUTLET & -0.173 & -0.212 & -0.190 \\
\hline & $\mathrm{C}_{\text {PO")TOTAL }}$ & -0.156 & -0.200 & -0.178 \\
\hline & C $_{\text {PO'JPROFILE }}$ & -0.081 & -0.122 & -0.083 \\
\hline & $\mathrm{C}_{\mathrm{PO}}{ }^{\prime \prime}$ )SECONDARY & -0.075 & -0.078 & -0.096 \\
\hline & $\alpha_{2}{ }^{\prime \prime}$ [deg] & -57.0 & -57.1 & -58.7 \\
\hline & $Y_{\text {TOTAL }}$ & 0.070 & 0.094 & 0.080 \\
\hline & $Y_{\text {PROFILE }}$ & 0.035 & 0.060 & 0.036 \\
\hline & $Y_{\text {SECONDARY }}$ & 0.035 & 0.034 & 0.044 \\
\hline & $\mathrm{Q}_{2} / \mathrm{Q}_{\mathrm{CL}}{ }^{\prime \prime}$ & 2.23 & 2.12 & 2.24 \\
\hline
\end{tabular}


Table D.2: Summary of Results at Design Incidence, FSTI $=\mathbf{4 . 0 \%}$

\begin{tabular}{|c|c|c|c|c|}
\hline & & PAKB & PAKD-A & PAKD-F \\
\hline \multirow{13}{*}{ 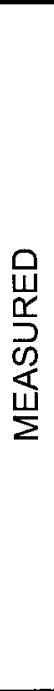 } & $\mathrm{C}_{\mathrm{PO}}{ }^{\prime \prime}$ )INLET & -0.036 & -0.035 & -0.035 \\
\hline & $\overline{C_{\text {POO JOUTLET }}}$ & -0.165 & -0.166 & -0.164 \\
\hline & $\mathrm{C}_{\mathrm{PO}}{ }^{\prime \prime}$ )TOTAL & -0.130 & -0.131 & -0.129 \\
\hline & $\mathrm{Y}_{\text {TOTAL }}$ & 0.054 & 0.057 & 0.055 \\
\hline & $Y_{\text {PROFILE }}$ & 0.035 & 0.040 & 0.031 \\
\hline & $Y_{\text {SECONDARY }}$ & 0.019 & 0.017 & 0.024 \\
\hline & $\mathrm{C}_{\Gamma+}$ & 0.338 & 0.377 & 0.406 \\
\hline & $\mathrm{C}_{\Gamma-}$ & -0.198 & -0.206 & -0.294 \\
\hline & $\mathrm{C}_{\text {SKE }}{ }^{\prime \prime}$ & 0.015 & 0.014 & 0.024 \\
\hline & $\alpha_{2}{ }^{\prime \prime}$ [deg] & -56.1 & -56.4 & -56.5 \\
\hline & $\mathrm{Q}_{2} / \mathrm{Q}_{\mathrm{CL}}{ }^{\prime \prime}$ & 2.39 & 2.31 & 2.34 \\
\hline & AVR' & 1.00 & 1.00 & 0.98 \\
\hline & $Q$ & 4.7 & 6.1 & 6.7 \\
\hline \multirow{9}{*}{ 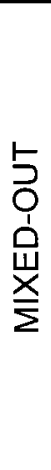 } & $\mathrm{C}_{\mathrm{PO}}$ "JOUTLET & -0.186 & -0.188 & -0.197 \\
\hline & $\mathrm{C}_{\text {P" }}{ }^{\prime \prime}$ TOTAL & -0.150 & -0.154 & -0.162 \\
\hline & C $_{\text {PO')PROFILE }}$ & -0.086 & -0.098 & -0.077 \\
\hline & $C_{P O}{ }^{\prime \prime}$ SECONDARY & -0.065 & -0.056 & -0.085 \\
\hline & $\alpha_{2}{ }^{\prime \prime}[\mathrm{deg}]$ & -56.6 & -56.9 & -57.2 \\
\hline & $Y_{\text {TOTAL }}$ & 0.064 & 0.068 & 0.071 \\
\hline & $Y_{\text {PROFILE }}$ & 0.036 & 0.042 & 0.032 \\
\hline & $Y_{\text {SECONDARY }}$ & 0.028 & 0.026 & 0.039 \\
\hline & $\mathrm{Q}_{2} / \mathrm{Q}_{\mathrm{CL}}{ }^{\prime \prime}$ & 2.34 & 2.26 & 2.27 \\
\hline
\end{tabular}




\section{Appendix E}

\section{Summary of Experimental Results at Off-Design Incidence}

This appendix provides the summary of mass-averaged results for all three airfoils at off-design incidence and two FSTIs. Tables E.1, and E.2 present the result summary for $-5^{\circ}$ and $+5^{\circ}$ at FSTI of $1.5 \%$, respectively. Similarly, Table E.3 presents the results at $+5^{\circ}$ at FSTI of $4.0 \%$.

The profile losses and midspan AVR were obtained from Popovic (2005). 
Table E.1: Summary of Results at $-5^{\circ}, \mathbf{F S T I}=\mathbf{1 . 5 \%}$

\begin{tabular}{|c|c|c|c|c|}
\hline & & PAKB & PAKD-A & PAKD-F \\
\hline \multirow{12}{*}{ 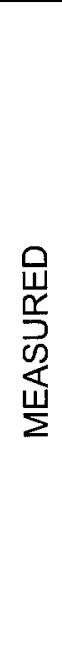 } & $\mathrm{C}_{\mathrm{PO}}{ }^{\prime \prime}$ )INLET & -0.008 & -0.009 & -0.009 \\
\hline & $\mathrm{C}_{\mathrm{PO}}$ "joutLeT & -0.150 & -0.163 & -0.140 \\
\hline & $\mathrm{C}_{\text {P0")TOTAL }}$ & -0.142 & -0.154 & -0.131 \\
\hline & $Y_{\text {TOTAL }}$ & 0.056 & 0.060 & 0.049 \\
\hline & Y PROFILE & 0.038 & 0.050 & 0.034 \\
\hline & $Y_{\text {SECONDARY }}$ & 0.018 & 0.010 & 0.015 \\
\hline & $\mathrm{C}_{\Gamma+}$ & 0.261 & 0.359 & 0.470 \\
\hline & $\mathrm{C}_{\mathrm{T}-\mathrm{-}}$ & -0.195 & -0.209 & -0.369 \\
\hline & $\mathrm{C}_{\text {SKE }}$ & 0.014 & 0.016 & 0.032 \\
\hline & $\alpha_{2}^{\prime \prime}$ & -56.0 & -54.4 & -56.4 \\
\hline & $\mathrm{Q}_{2} / \mathrm{Q}_{\mathrm{CL}}{ }^{\prime \prime}$ & 2.53 & 2.56 & 2.70 \\
\hline & AVR' & 0.99 & 0.99 & 0.98 \\
\hline \multirow{9}{*}{ 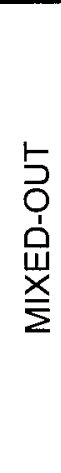 } & $\mathrm{C}_{\mathrm{PO}}$ ")OUTLET & -0.168 & -0.201 & -0.184 \\
\hline & $\mathrm{C}_{\text {PO")TOTAL }}$ & -0.159 & -0.192 & -0.175 \\
\hline & $\mathrm{C}_{\mathrm{PO} \text { ')PROFILE }}$ & -0.106 & -0.129 & -0.095 \\
\hline & CPO")SECONDARY $_{\text {P }}$ & -0.053 & -0.063 & -0.081 \\
\hline & $\alpha_{2} "$ & -56.4 & -55.1 & -57.2 \\
\hline & $Y_{\text {TOTAL }}$ & 0.065 & 0.078 & 0.067 \\
\hline & $Y_{\text {PROFILE }}$ & 0.039 & 0.051 & 0.035 \\
\hline & $Y_{\text {SECONDARY }}$ & 0.026 & 0.027 & 0.032 \\
\hline & $\mathrm{Q}_{2} / \mathrm{Q}_{\mathrm{CL}}{ }^{\prime \prime}$ & 2.49 & 2.48 & 2.61 \\
\hline
\end{tabular}


Table E.2: Summary of Results at $+5^{\circ}$, FSTI $=1.5 \%$

\begin{tabular}{|c|c|c|c|c|}
\hline & & PAKB & PAKD-A & PAKD-F \\
\hline \multirow{12}{*}{ 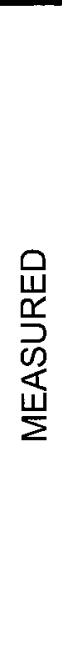 } & $\left.\mathrm{C}_{P O}{ }^{\prime \prime}\right)$ INLET & -0.011 & -0.014 & -0.012 \\
\hline & $\mathrm{C}_{\text {PO"JOUTLET }}$ & -0.135 & -0.643 & -0.162 \\
\hline & $\mathrm{C}_{\text {PO")TOTAL }}$ & -0.124 & -0.629 & -0.150 \\
\hline & $Y_{\text {TOTAL }}$ & 0.061 & 0.340 & 0.072 \\
\hline & YROFILE $_{\text {PR }}$ & 0.038 & 0.519 & 0.049 \\
\hline & $Y_{\text {SECONDARY }}$ & 0.023 & -0.179 & 0.023 \\
\hline & $\mathrm{C}_{\Gamma+}$ & 0.375 & 0.623 & 0.567 \\
\hline & $\mathrm{C}_{\Gamma-}$ & -0.307 & -0.286 & -0.452 \\
\hline & $\mathrm{C}_{\mathrm{SKE}} "$ & 0.027 & 0.030 & 0.044 \\
\hline & $\alpha_{2}^{\prime \prime}$ & -56.6 & -53.1 & -55.3 \\
\hline & $\mathrm{Q}_{2} / \mathrm{Q}_{\mathrm{CL}}{ }^{\prime \prime}$ & 2.048 & 1.849 & 2.064 \\
\hline & AVR' & 0.99 & 1.01 & 1.00 \\
\hline \multirow{9}{*}{ 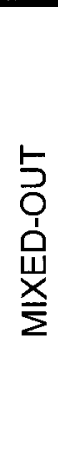 } & $\mathrm{C}_{\mathrm{PO}}{ }^{\prime \prime}$ OUTLET & -0.171 & -0.704 & -0.227 \\
\hline & $\mathrm{C}_{\mathrm{PO}}{ }^{\prime \prime}$ )TOTAL & -0.160 & -0.690 & -0.214 \\
\hline & C $_{\text {PO')PROFILE }}$ & -0.082 & -0.933 & -0.108 \\
\hline & $\mathrm{C}_{\mathrm{PO}}{ }^{\prime \prime}$ )SECONDARY & -0.078 & 0.243 & -0.107 \\
\hline & $\alpha_{2}^{\prime \prime}$ & -57.4 & -54.9 & -56.8 \\
\hline & $Y_{\text {TOTAL }}$ & 0.081 & 0.405 & 0.110 \\
\hline & Y PROFILE & 0.040 & 0.628 & 0.052 \\
\hline & $Y_{\text {SECONDARY }}$ & 0.041 & -0.223 & 0.058 \\
\hline & $Q_{2} / Q_{C L}{ }^{\prime \prime}$ & 1.98 & 1.70 & 1.94 \\
\hline
\end{tabular}


Table E.3: Summary of Results at $+5^{\circ}$, FSTI $=\mathbf{4 . 0 \%}$

\begin{tabular}{|c|c|c|c|c|}
\hline & & PAKB & PAKD-A & PAKD-F \\
\hline \multirow{12}{*}{ 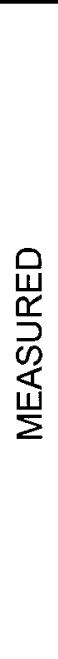 } & $\mathrm{C}_{\mathrm{PO}}{ }^{\prime \prime}$ )INLET & -0.029 & -0.031 & -0.030 \\
\hline & $\mathrm{C}_{\mathrm{PO} \text { JOUTLET }}$ & -0.165 & -0.204 & -0.193 \\
\hline & $\mathrm{C}_{\mathrm{PO}}{ }^{\prime \prime}$ )TOTAL & -0.136 & -0.173 & -0.162 \\
\hline & $Y_{\text {TOTAL }}$ & 0.067 & 0.087 & 0.078 \\
\hline & $\mathrm{Y}_{\text {PROFILE }}$ & 0.041 & 0.064 & 0.047 \\
\hline & $Y_{\text {SECONDARY }}$ & 0.026 & 0.023 & 0.031 \\
\hline & $\mathrm{C}_{\Gamma+}$ & 0.346 & 0.441 & 0.449 \\
\hline & $\mathrm{C}_{\Gamma-}$ & -0.234 & -0.314 & -0.355 \\
\hline & $\mathrm{C}_{\text {SKE" }}$ & 0.018 & 0.022 & 0.028 \\
\hline & $\alpha_{2}^{\prime \prime}$ & -56.2 & -55.7 & -56.1 \\
\hline & $\mathrm{Q}_{2} / \mathrm{Q}_{\mathrm{CL}}{ }^{\prime \prime}$ & 2.027 & 1.983 & 2.086 \\
\hline & AVR' & 1.01 & 1.00 & 0.99 \\
\hline \multirow{9}{*}{ 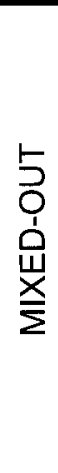 } & $\mathrm{C}_{\mathrm{PO}}$ ")OUTLET & -0.188 & -0.237 & -0.231 \\
\hline & $\mathrm{C}_{\text {PO")TOTAL }}$ & -0.159 & -0.206 & -0.201 \\
\hline & C $_{\text {PO'JPROFILE }}$ & -0.088 & -0.132 & -0.105 \\
\hline & $\mathrm{C}_{\mathrm{PO}}{ }^{\prime}$ )SECONDARY & -0.071 & -0.075 & -0.096 \\
\hline & $\alpha_{2}{ }^{\prime \prime}$ & -56.8 & -56.6 & -56.9 \\
\hline & $Y_{\text {TOTAL }}$ & 0.080 & 0.108 & 0.100 \\
\hline & $\mathrm{Y}_{\text {PROFILE }}$ & 0.043 & 0.067 & 0.050 \\
\hline & $Y_{\text {SECONDARY }}$ & 0.037 & 0.041 & 0.050 \\
\hline & $\mathrm{Q}_{2} / \mathrm{Q}_{\mathrm{CL}}{ }^{\prime \prime}$ & 1.98 & 1.91 & 2.01 \\
\hline
\end{tabular}




\section{Appendix F}

\section{Total Pressure Distribution}

The total pressure coefficient, $C_{P 0}$, contours for all three blades at design and offdesign incidences and both FSTIs are presented in this appendix. The total pressure coefficient was defined in equation 3.6 in Chapter 3 and its contours were discussed in greater detail in Chapters 4 and 5 .

The appendix begins with comparison of $C_{P 0}$ contours for the PAKB blade at three incidences examined $\left(-5^{\circ}, 0^{\circ},+5^{\circ}\right)$ and FSTI of $1.5 \%$. It continues with comparison of PAKB contours for two FSTIs (1.5\% and $4.0 \%)$ at design and positive off-design incidence. The same scheme is followed for the remaining two blades, PAKD-A, and PAKD-F. Lastly all three blades are compared at all incidences and FSTIs examined. 


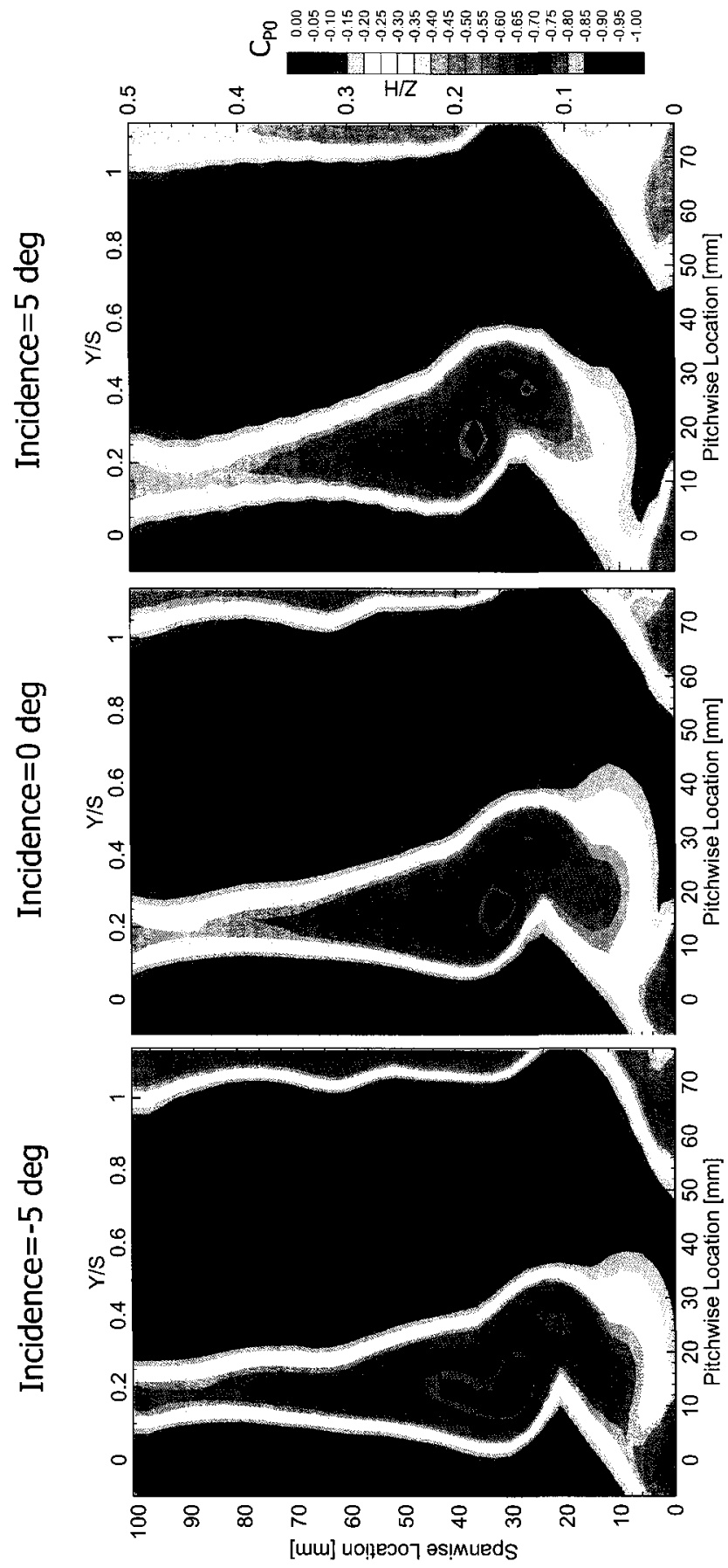

Figure F.1: PAKB: Total Pressure Contours at Design and Off-Design Incidence at $\mathrm{FSTI}=1.5 \%$ 

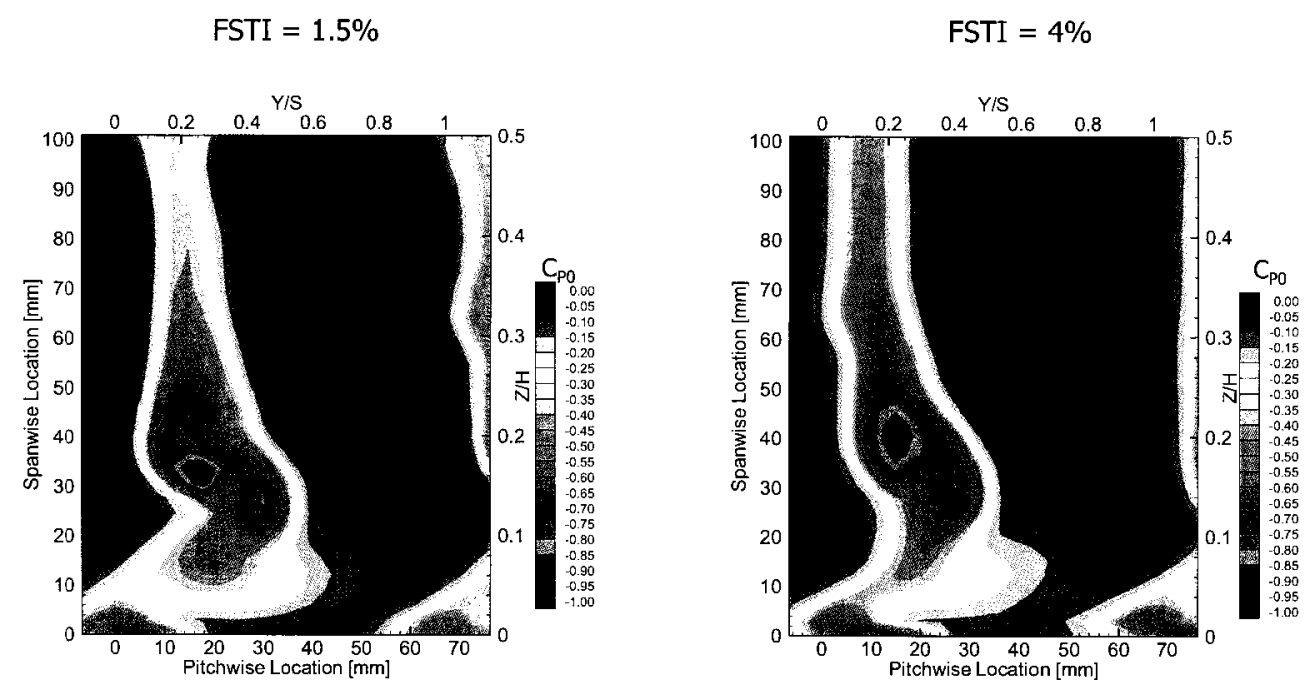

Figure F.2: PAKB: Total Pressure Contours at Design Incidence at FSTI=1.5\% and $4.0 \%$

$\mathrm{FSTI}=1.5 \%$

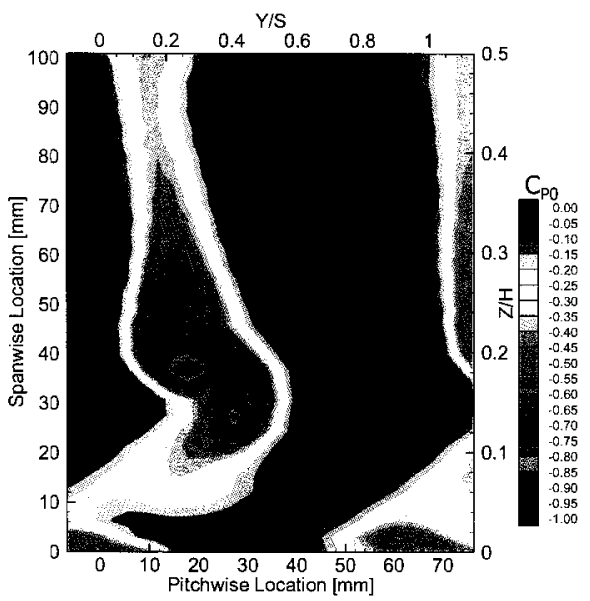

$\mathrm{FSTI}=4 \%$

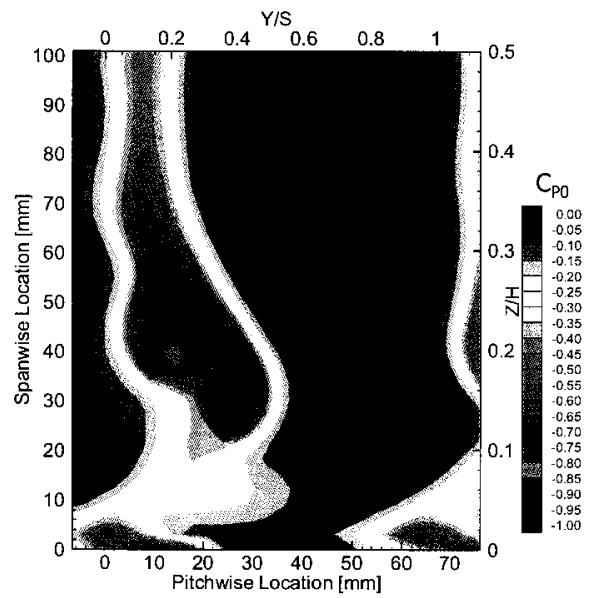

Figure F.3: PAKB: Total Pressure Contours at $+5^{\circ}$ Incidence at FSTI $=1.5 \%$ and $4.0 \%$ 

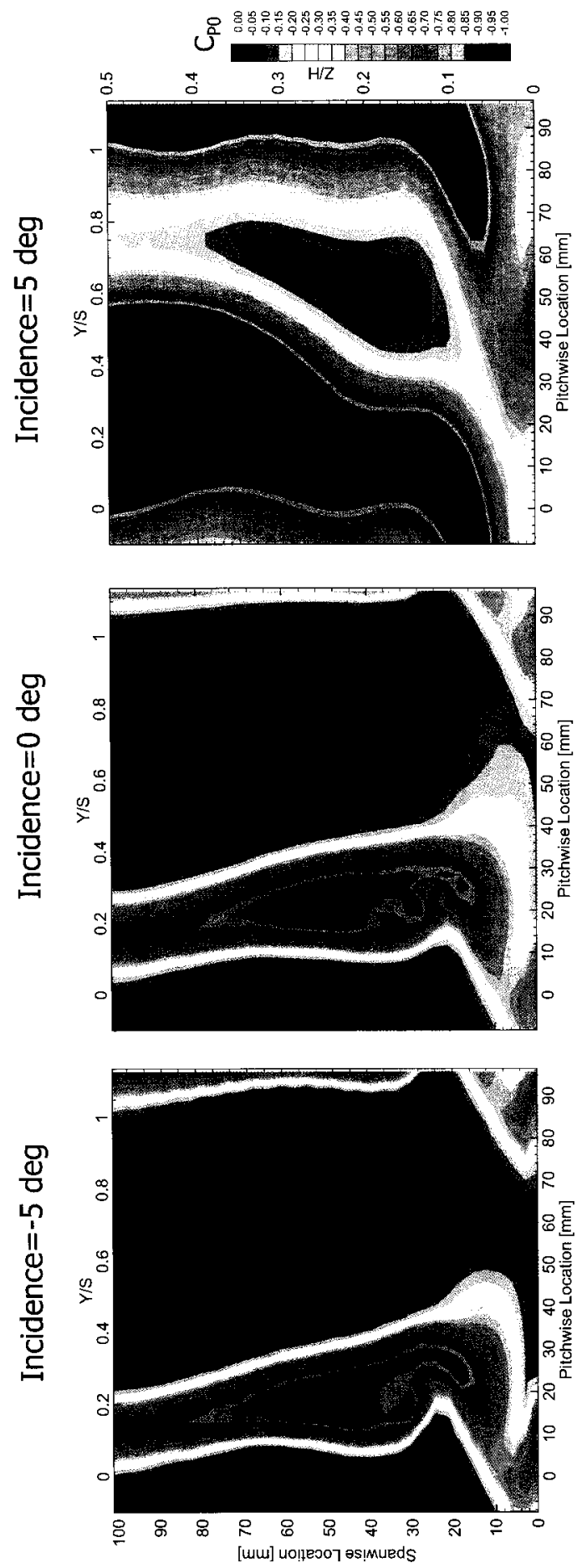

Figure F.4: PAKD-A: Total Pressure Contours at Design and Off-Design Incidence at $\mathbf{F S T I}=1.5 \%$ 

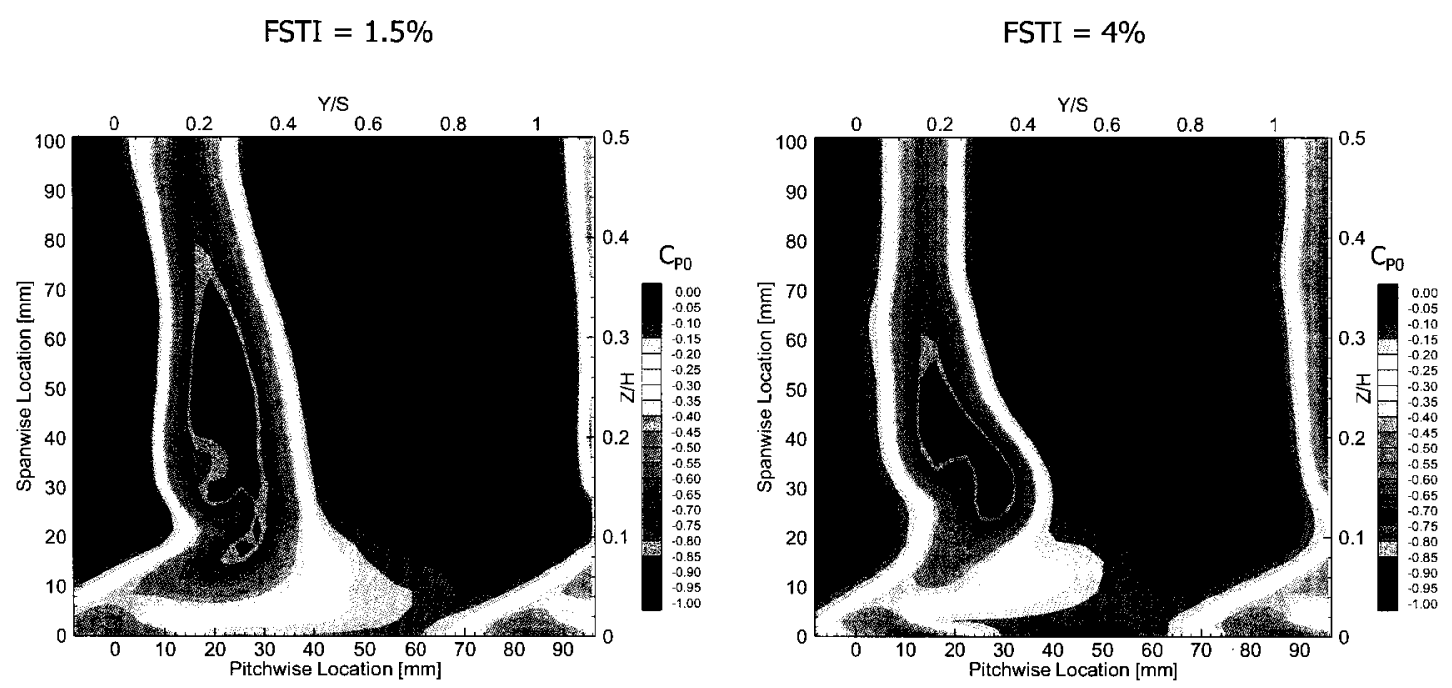

Figure F.5: PAKD-A: Total Pressure Contours at Design Incidence at FSTI $=1.5 \%$ and $4.0 \%$

$\mathrm{FSTI}=1.5 \%$

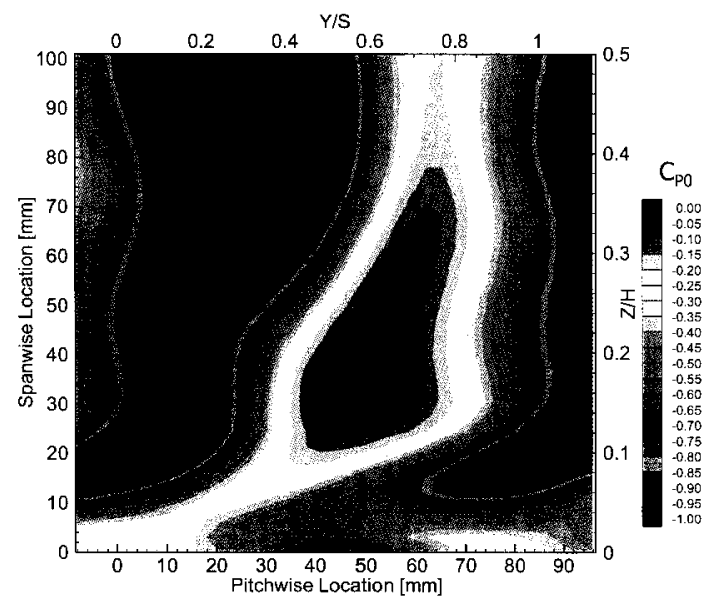

$\mathrm{FSTI}=4 \%$

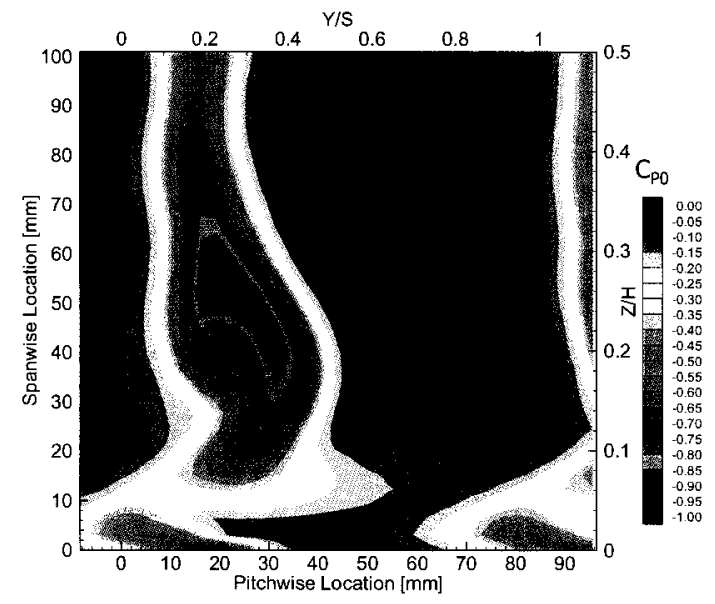

Figure F.6: PAKD-A: Total Pressure Contours at $+5^{\circ}$ Incidence at FSTI $=1.5 \%$ and $4.0 \%$ 

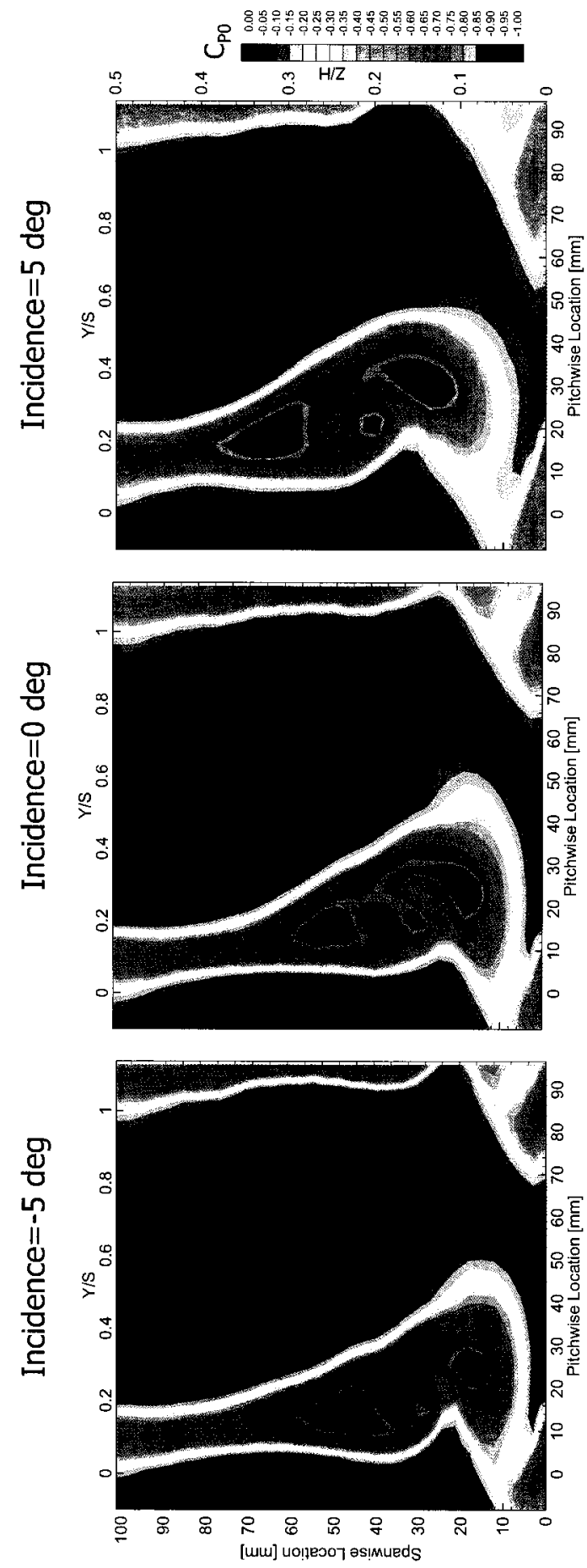

Figure F.7: PAKD-F: Total Pressure Contours at Design and Off-Design Incidence at FSTI $=1.5 \%$ 

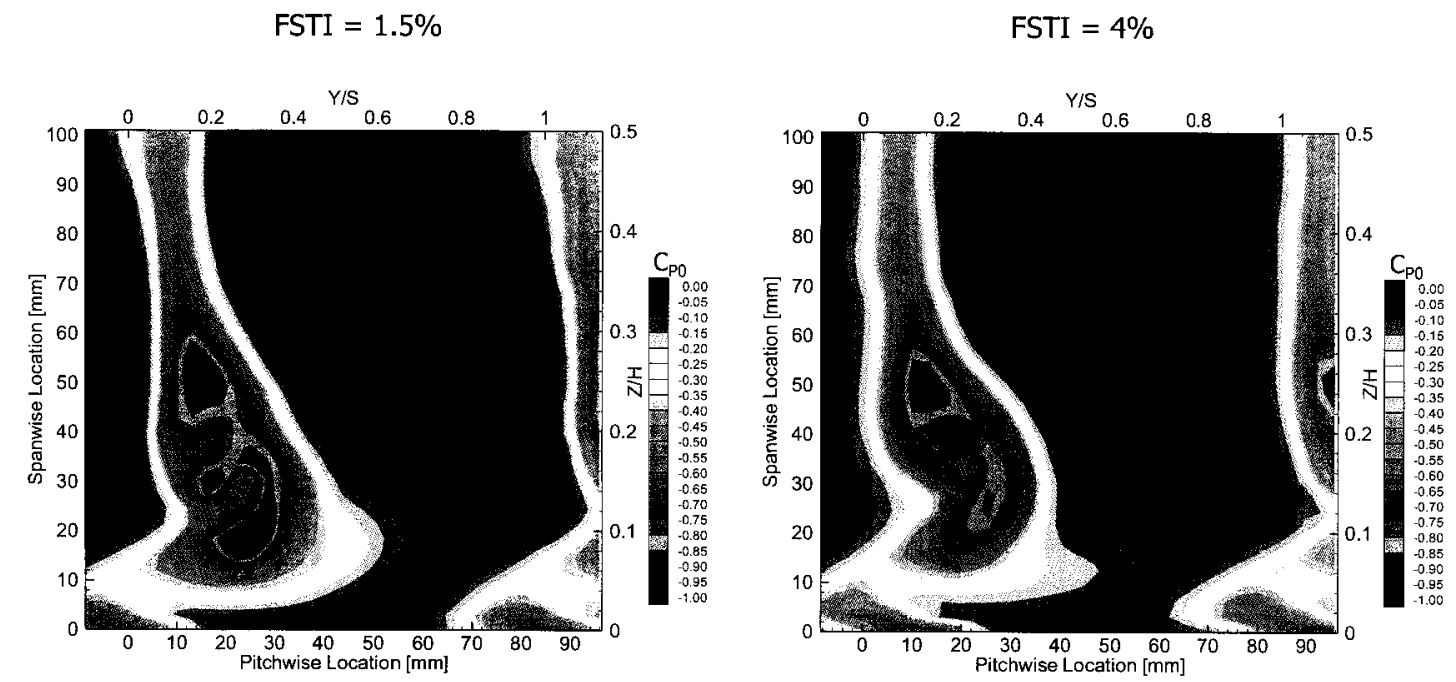

Figure F.8: PAKD-F: Total Pressure Contours at Design Incidence at FSTI $=1.5 \%$ and $4.0 \%$

$\mathrm{FSTI}=1.5 \%$

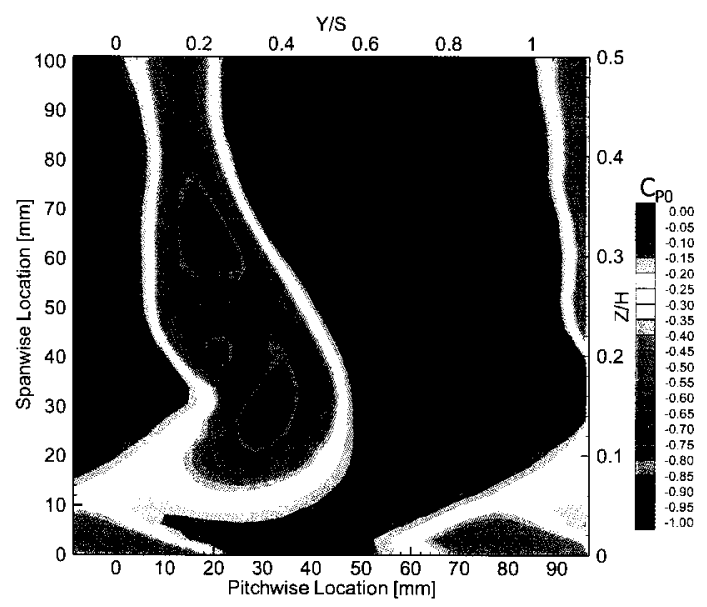

$\mathrm{FSTI}=4 \%$

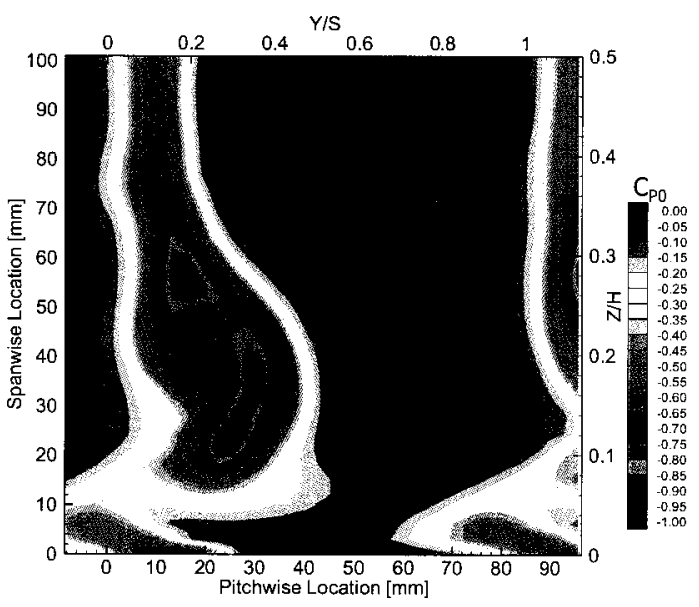

Figure F.9: PAKD-F: Total Pressure Contours at $+5^{\circ}$ Incidence at FSTI $=1.5 \%$ and $4.0 \%$ 

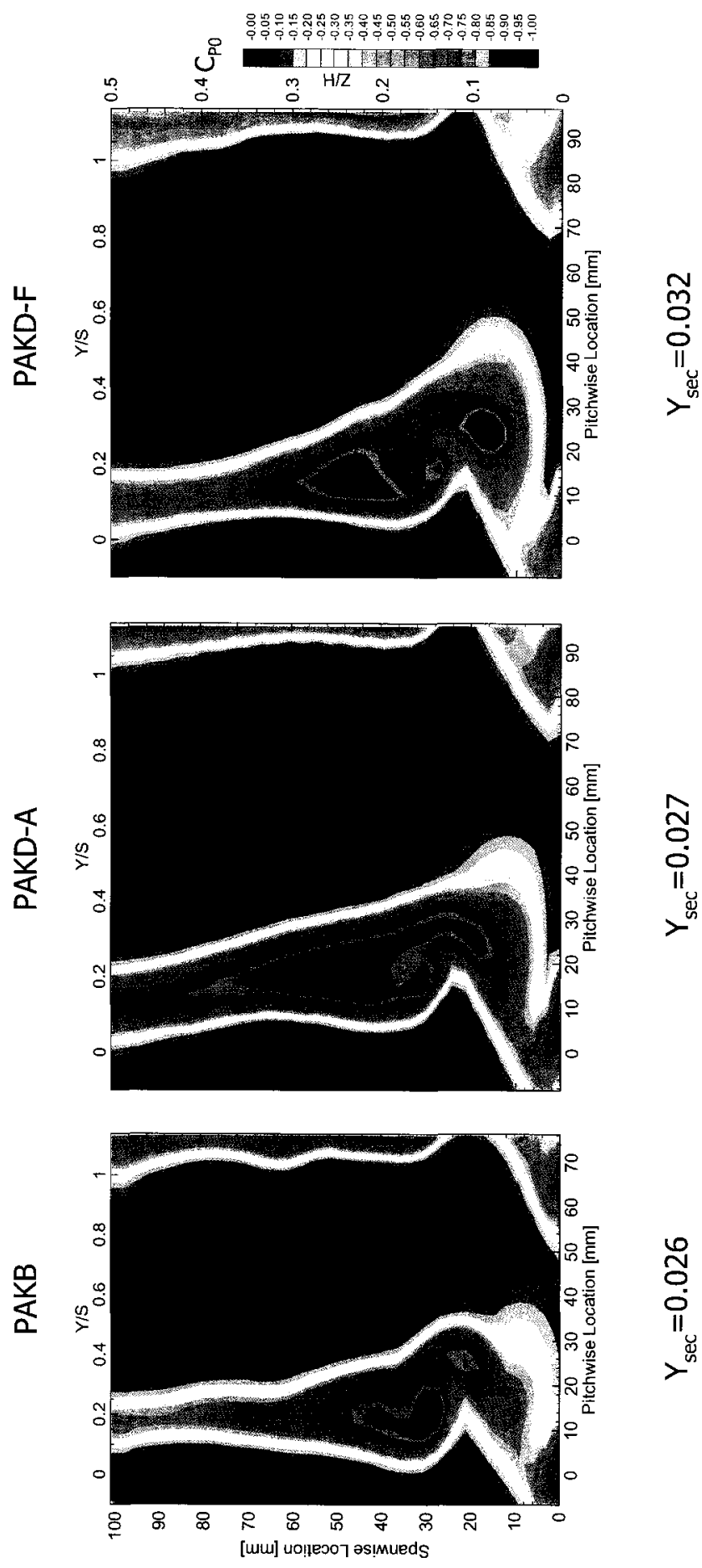

Figure F.10: Total Pressure Contours at $-5^{\circ}$ Incidence at FSTI $=1.5 \%$ 

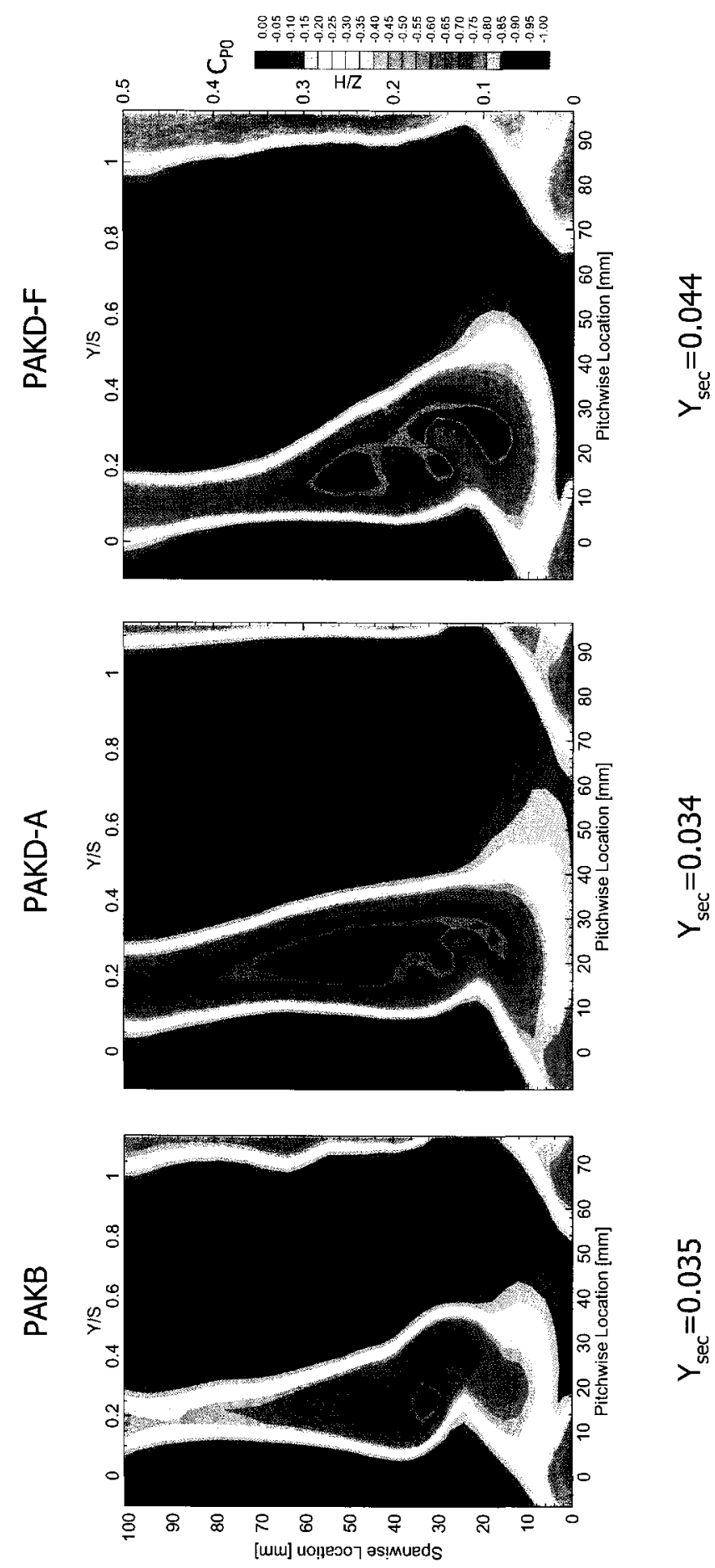

Figure F.11: Total Pressure Contours at $0^{\circ}$ Incidence at FSTI $=\mathbf{1 . 5 \%}$ 

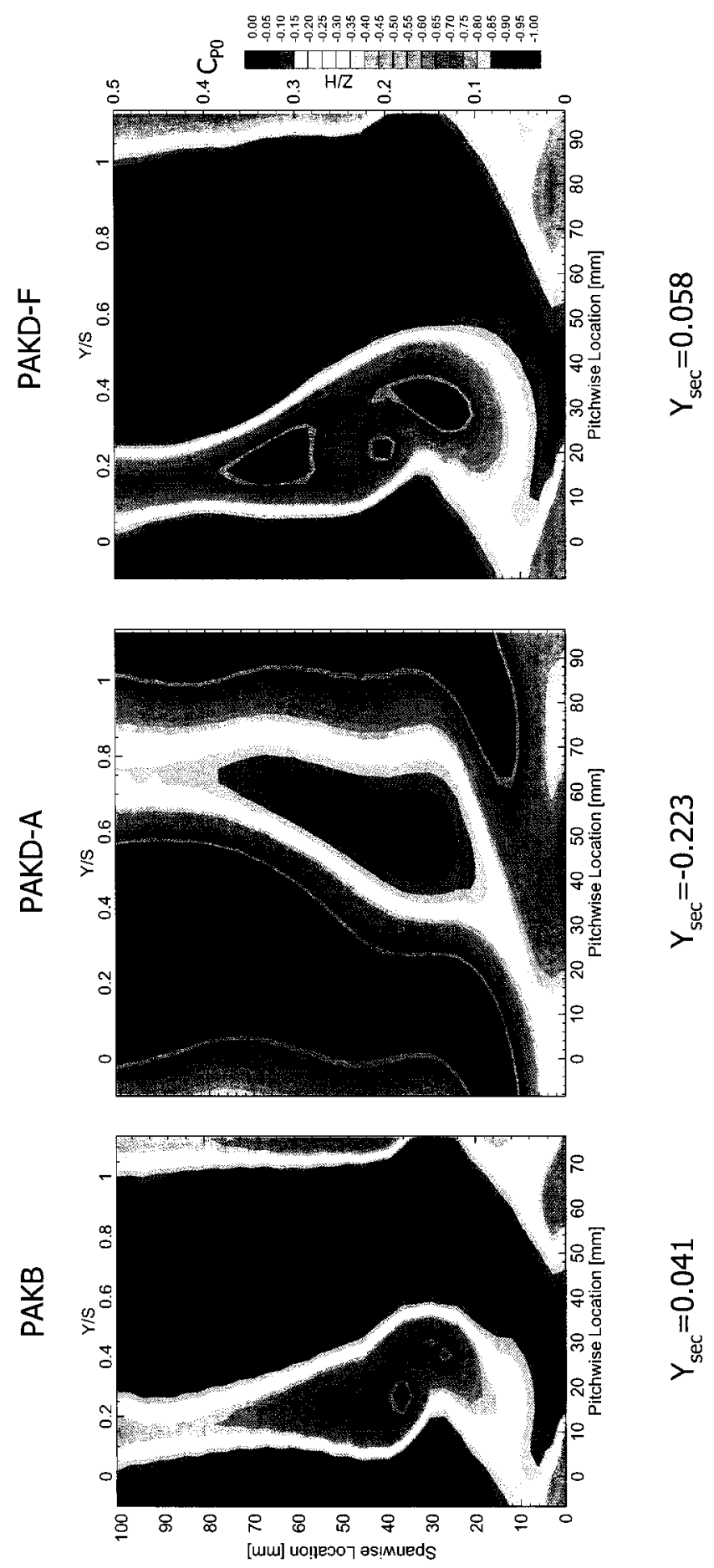

Figure F.12: Total Pressure Contours at $+5^{\circ}$ Incidence at FSTI $=1.5 \%$ 

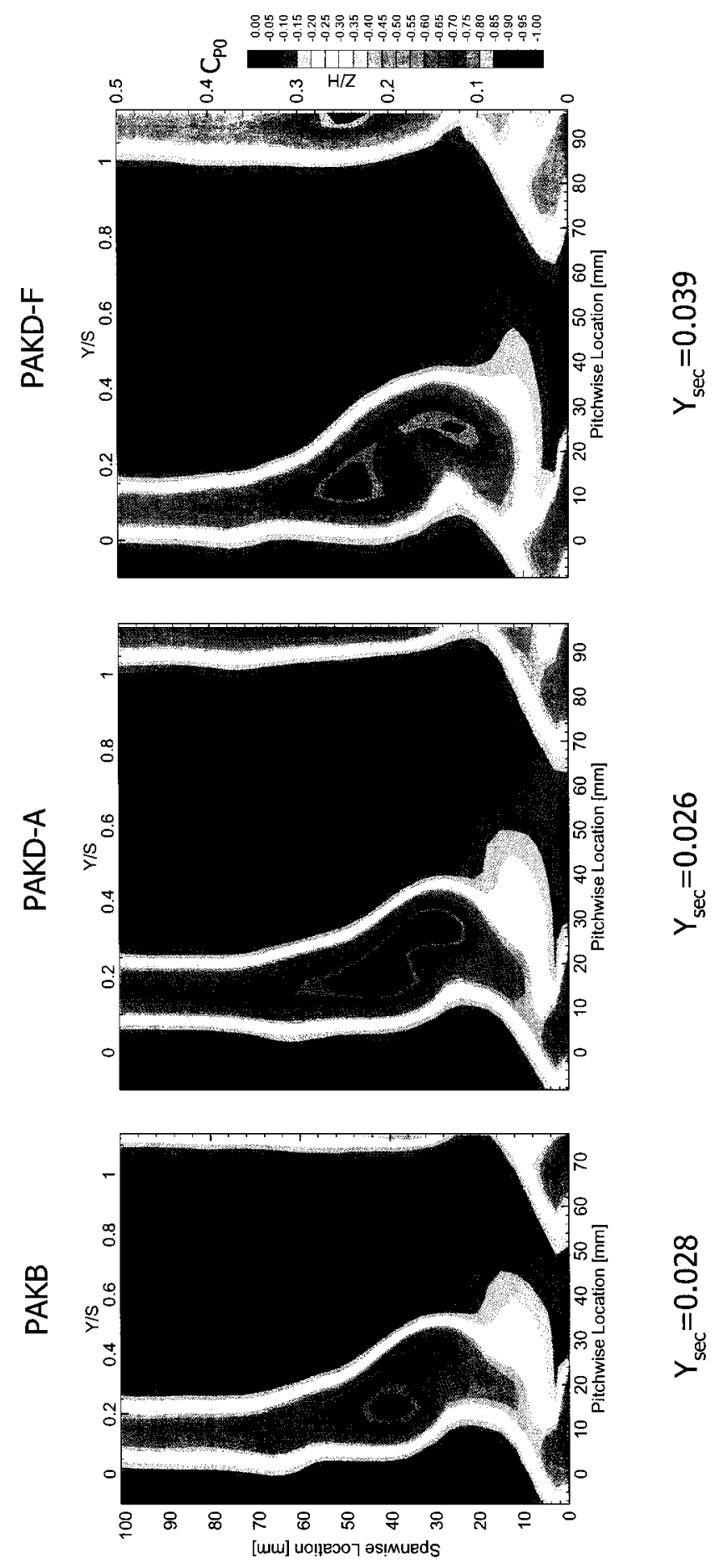

Figure F.13: Total Pressure Contours at $0^{\circ}$ Incidence at FSTI $=4.0 \%$ 

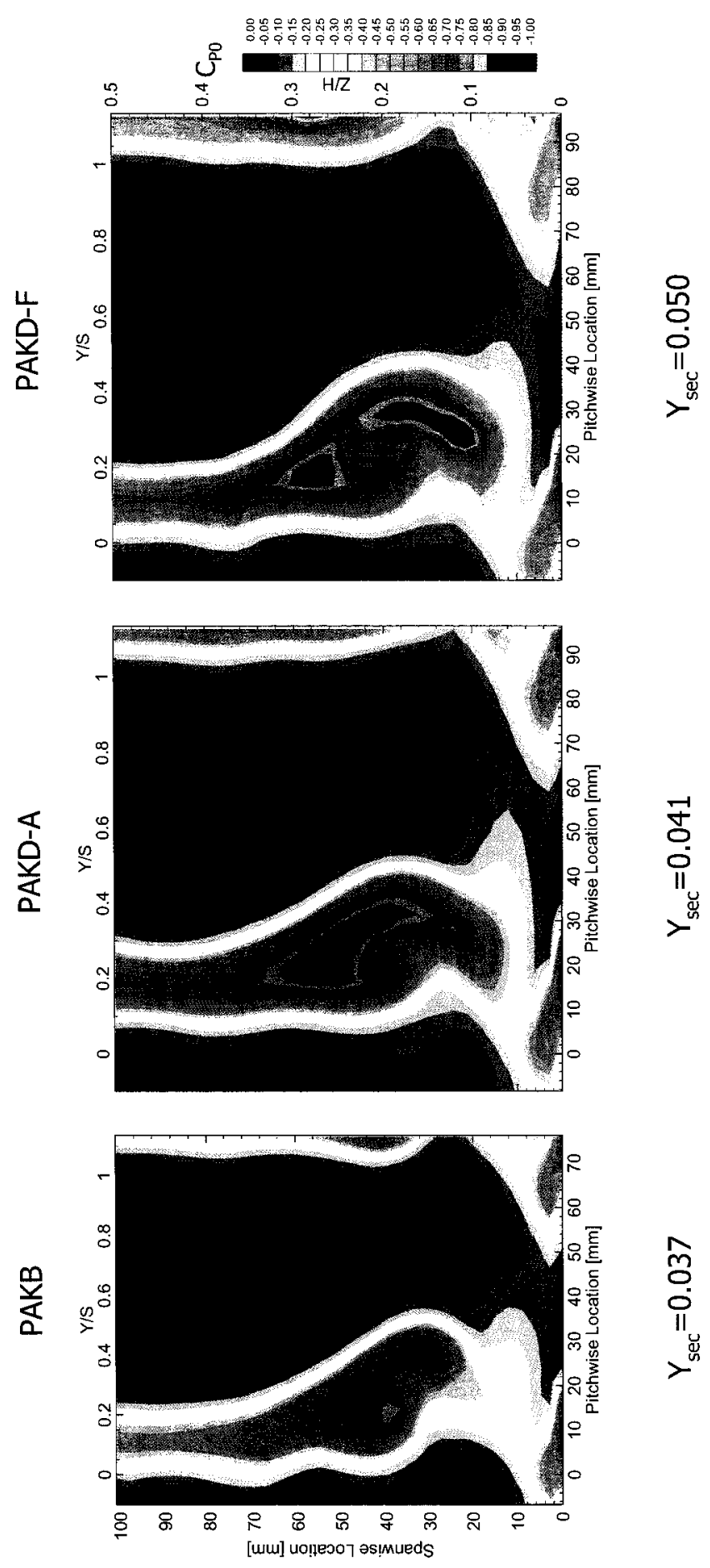

Figure F.14: Total Pressure Contours at $+5^{\circ}$ Incidence at FSTI $=4.0 \%$ 


\section{Appendix G}

\section{Positive Vorticity}

This appendix presents the positive vorticity superimposed with total pressure coefficient line contours. Streamwise vorticity was introduced in Chapter 3 and was later discussed in Chapters 4 and 5. As was previously mentioned, positive vorticity is rotating counterclockwise and is associated with counter and corner vortices. The corner vortex is located in the corner between the blade suction surface and the endwall, whereas the counter vortex is also located close to the suction surface, but farther away from the endwall and closer to the blade midspan.

The appendix is organized in the same way as the previous one. It begins with comparison of positive non-dimensional vorticity, $C_{\omega}$, contours for the PAKB blade at three incidences examined $\left(-5^{\circ}, 0^{\circ},+5^{\circ}\right)$ and FSTI of $1.5 \%$. It continues with comparison of PAKB contours for two FSTIs (1.5\% and 4.0\%) at design and positive off-design incidence. The same scheme is followed for the remaining two blades, PAKD-A, and PAKD-F. Lastly all three blades are compared at all incidences and FSTIs examined. 

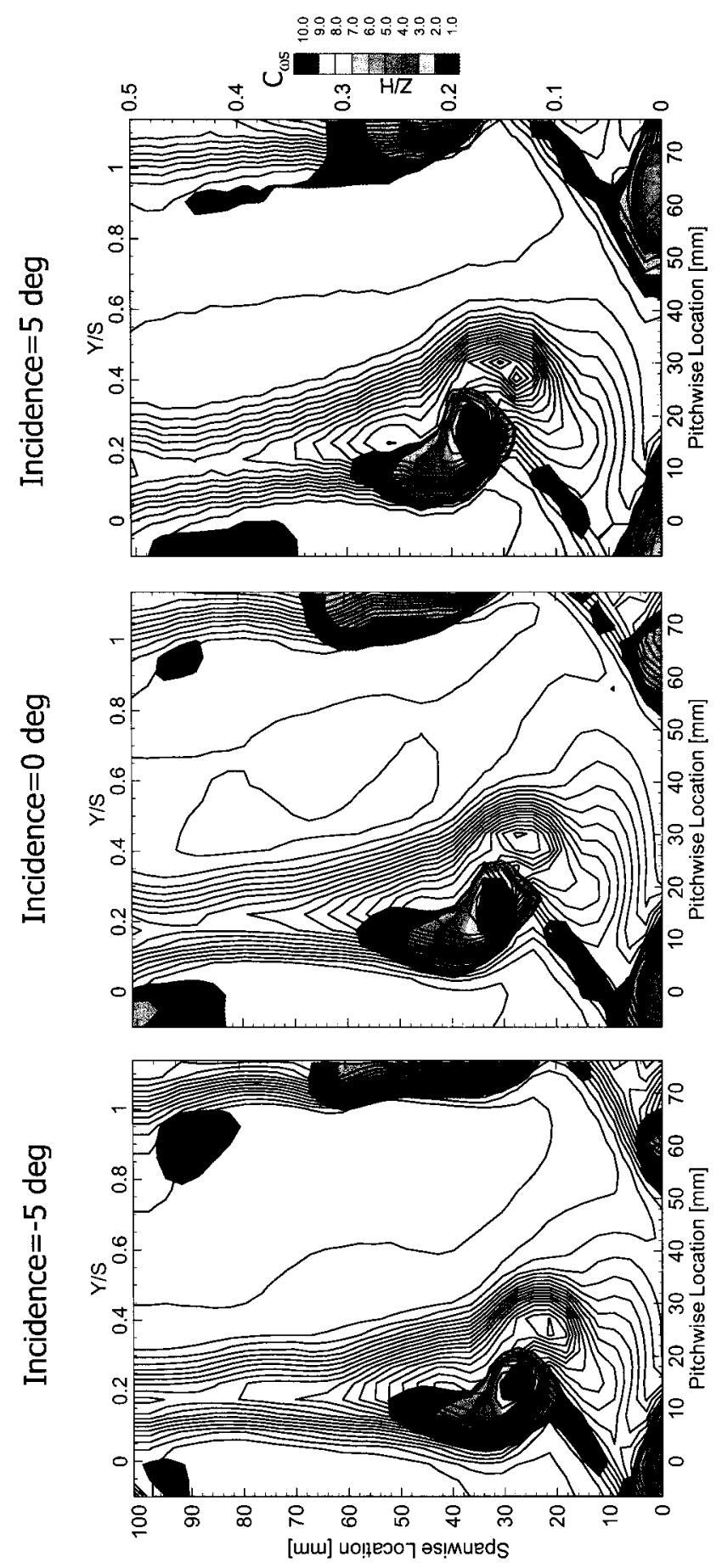

Figure G.1: PAKB - Line Contours of Total Pressure Coefficient Superimposed on Flood Contours of Positive Vorticity at Design and Off-Design Conditions at $\mathrm{FSTI}=1.5 \%$ 
$\mathrm{FSTI}=1.5 \%$

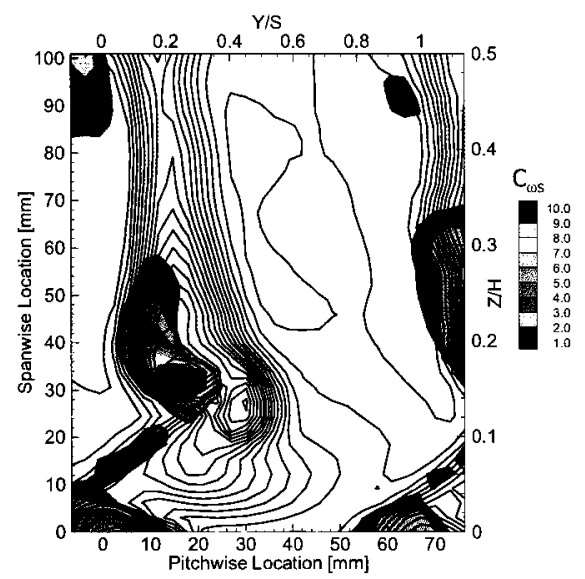

$\mathrm{FSTI}=4 \%$

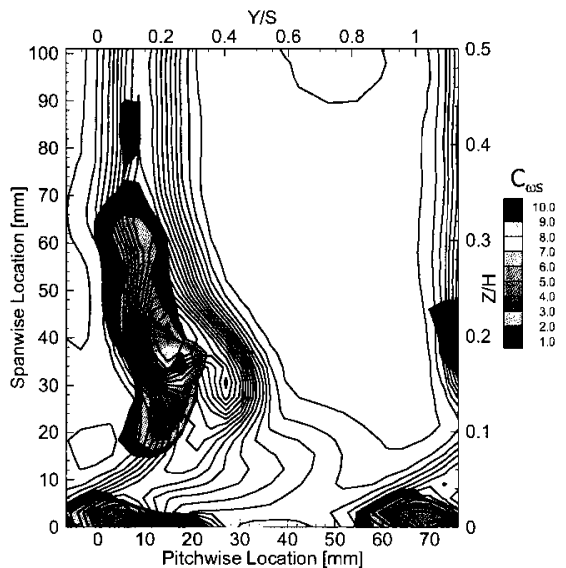

Figure G.2: PAKB - Line Contours of Total Pressure Coefficient Superimposed on Flood Contours of Positive Vorticity at Design Incidence at FSTI $=1.5 \%$ and $4.0 \%$

$\mathrm{FSTI}=1.5 \%$

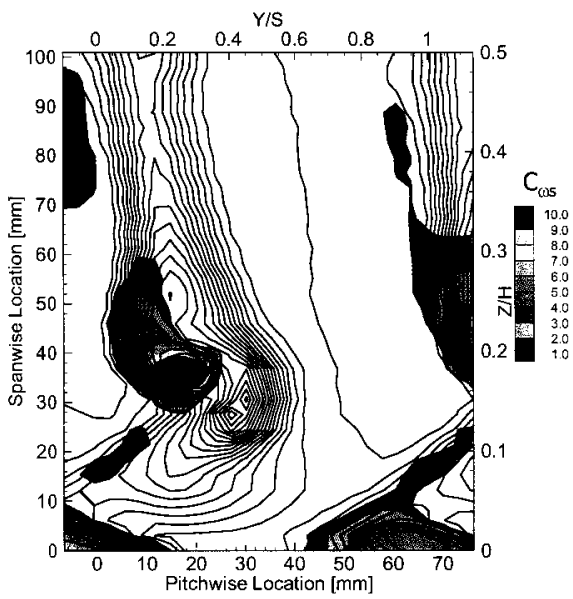

$\mathrm{FSTI}=4 \%$

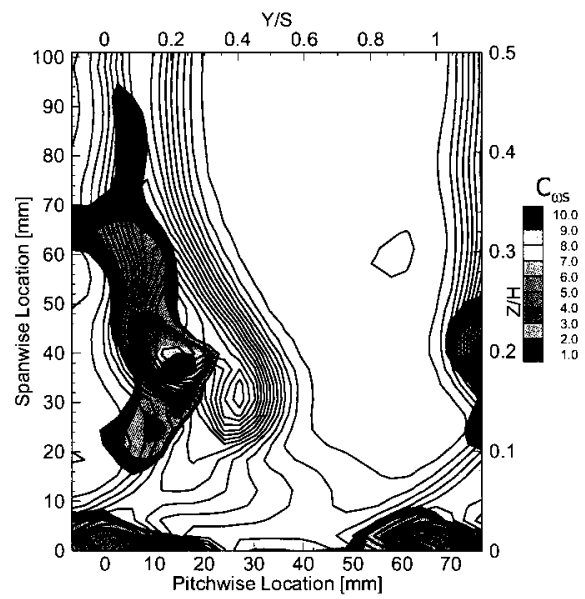

Figure G.3: PAKB - Line Contours of Total Pressure Coefficient Superimposed on Flood Contours of Positive Vorticity at $+5^{\circ}$ Incidence at FSTI $=1.5 \%$ and $4.0 \%$ 

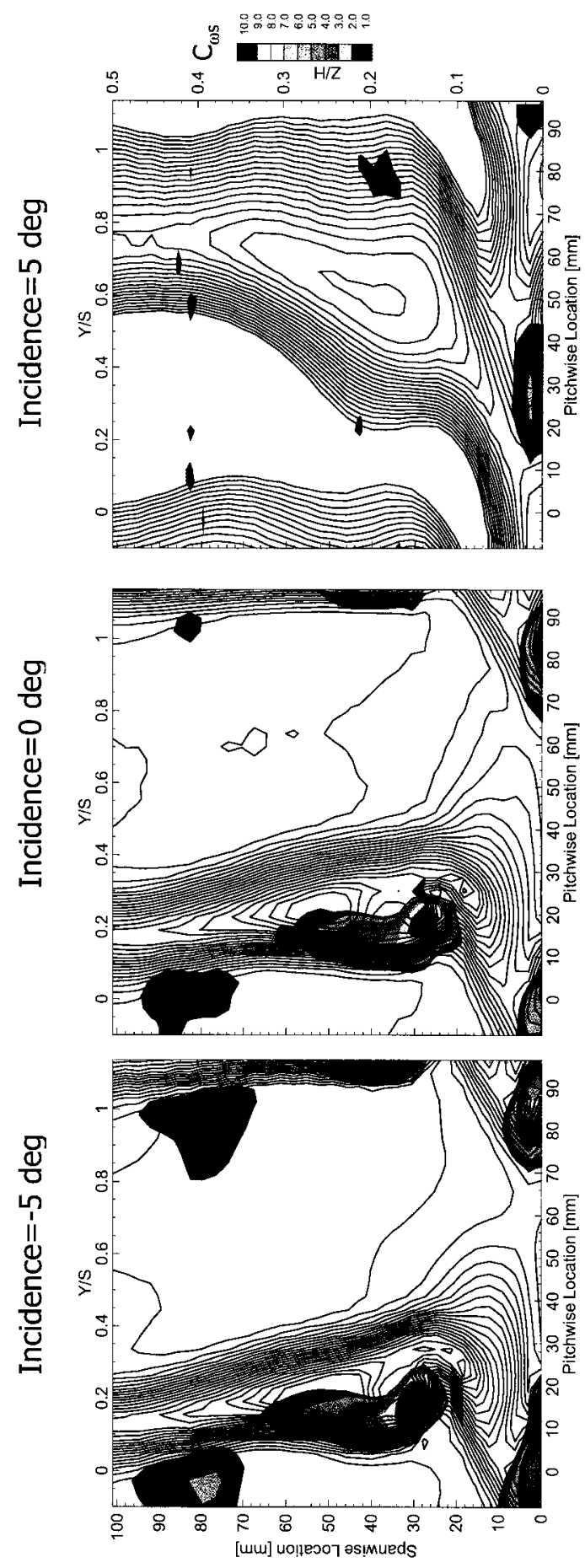

Figure G.4: PAKDA - Line Contours of Total Pressure Coefficient Superimposed on Flood Contours of Positive Vorticity at Design and Off-Design Conditions at $\mathrm{FSTI}=1.5 \%$ 

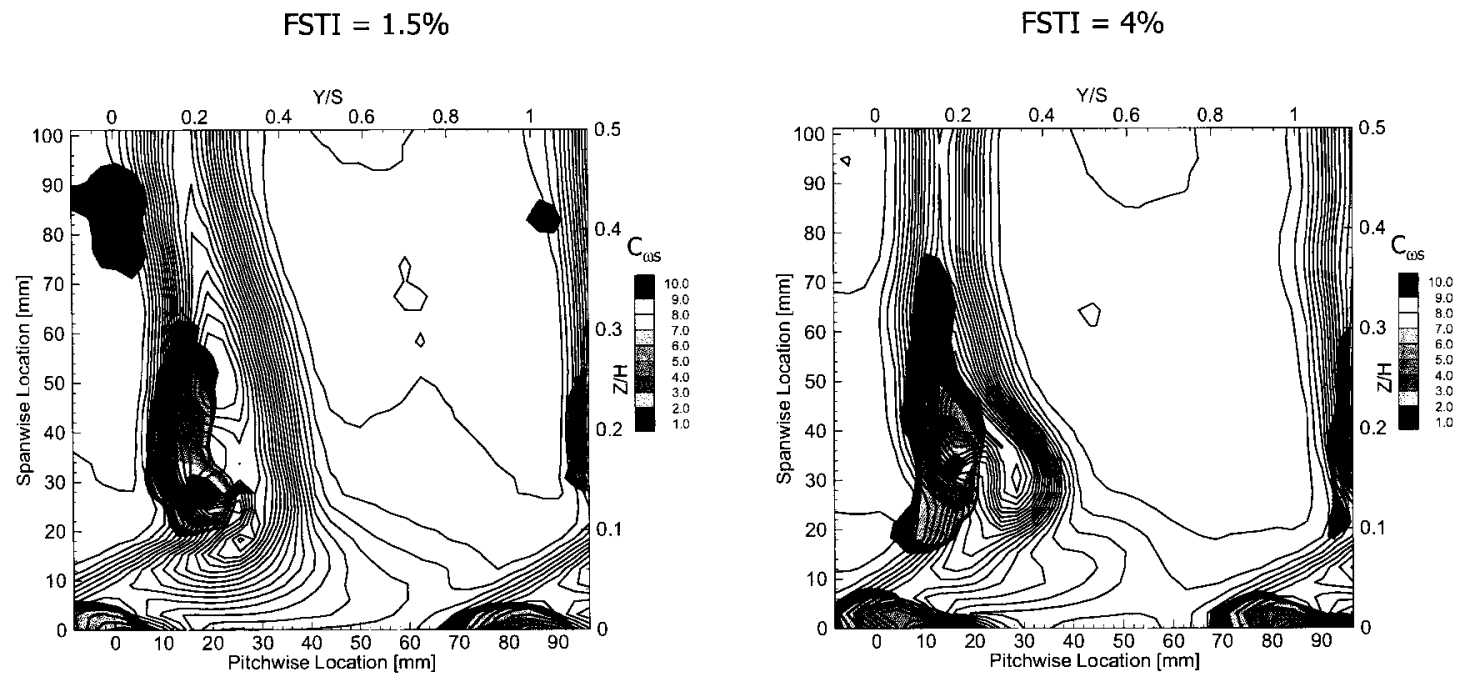

Figure G.5: PAKDA - Line Contours of Total Pressure Coefficient Superimposed on Flood Contours of Positive Vorticity at Design Incidence at FSTI $=1.5 \%$ and $4.0 \%$

$\mathrm{FSTI}=1.5 \%$

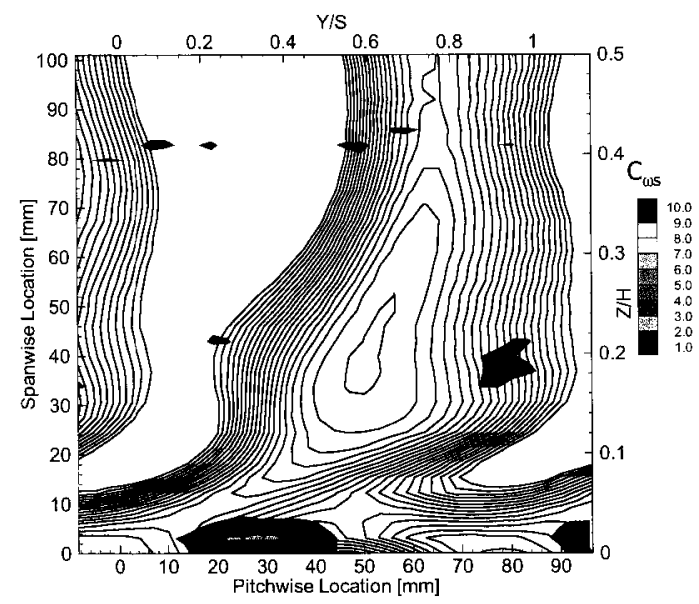

$\mathrm{FSTI}=4 \%$

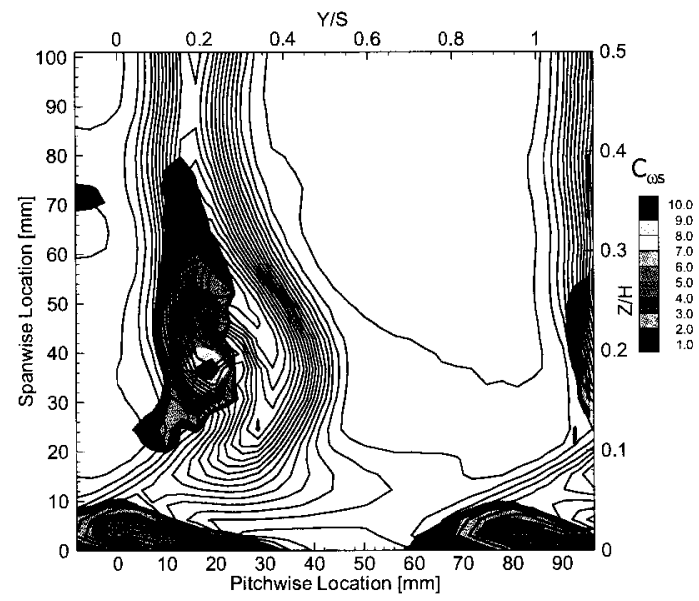

Figure G.6: PAKDA - Line Contours of Total Pressure Coefficient Superimposed on Flood Contours of Positive Vorticity at $+5^{\circ}$ Incidence at FSTI $=1.5 \%$ and $4.0 \%$ 

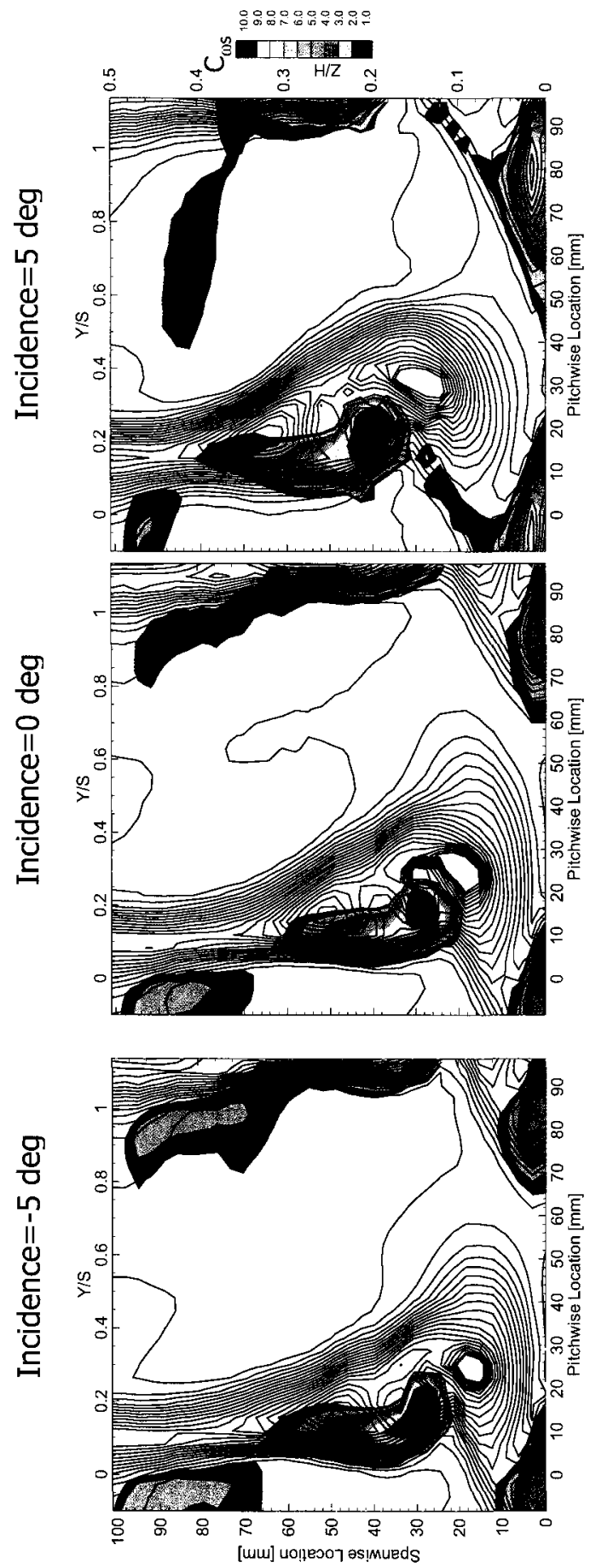

Figure G.7: PAKDF - Line Contours of Total Pressure Coefficient Superimposed on Flood Contours of Positive Vorticity at Design and Off-Design Conditions at $\mathrm{FSTI}=1.5 \%$ 

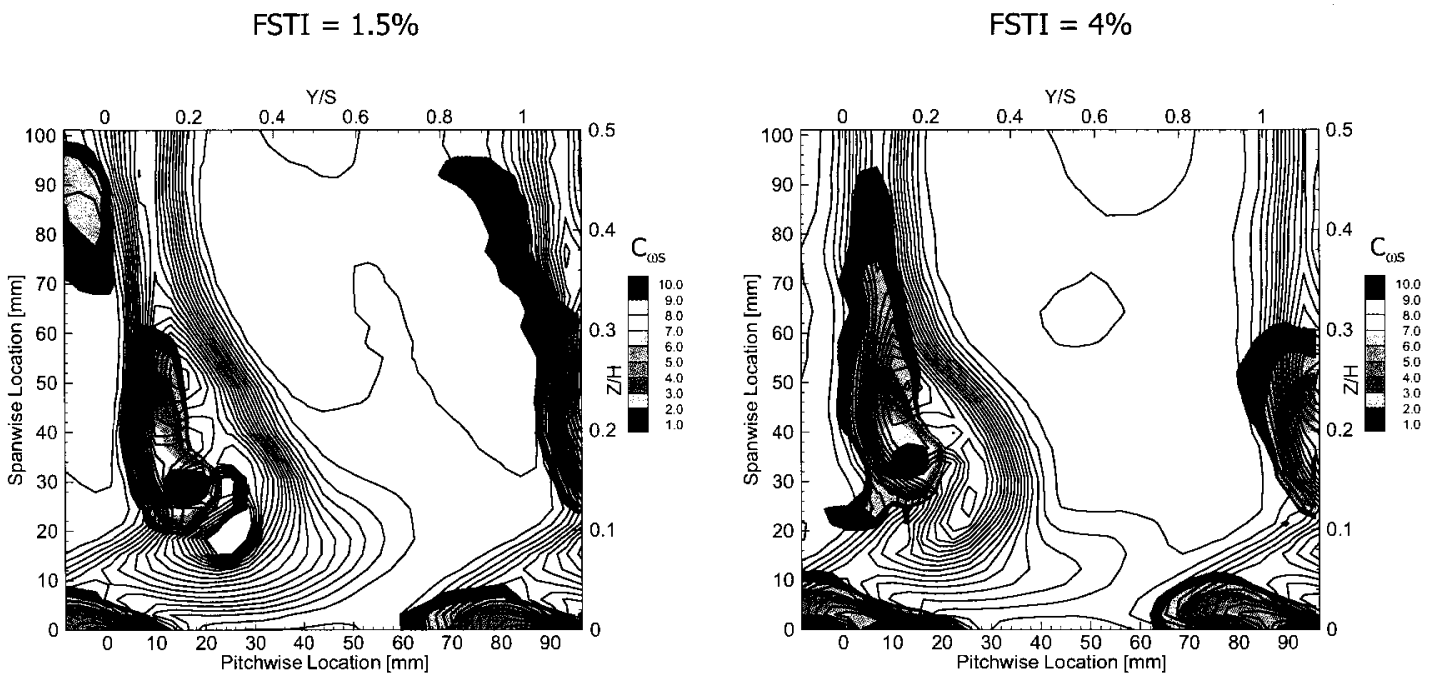

Figure G.8: PAKDF - Line Contours of Total Pressure Coefficient Superimposed on Flood Contours of Positive Vorticity at Design Incidence at FSTI $=1.5 \%$ and $4.0 \%$

$\mathrm{FSTI}=1.5 \%$

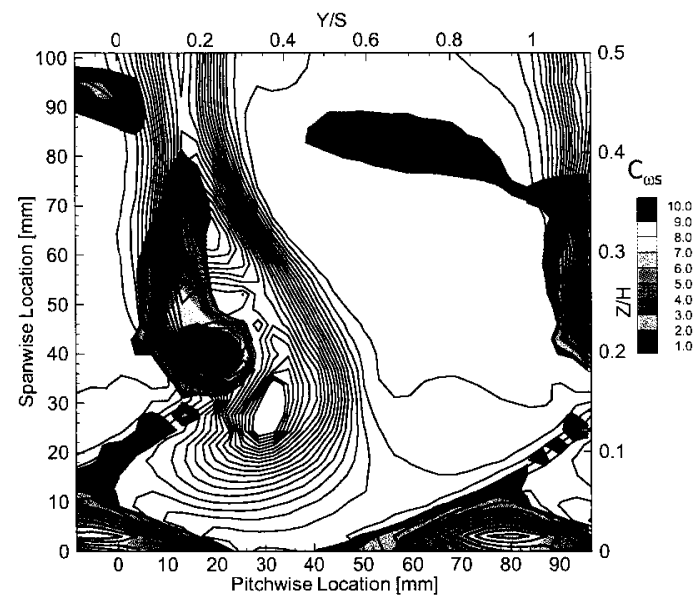

$\mathrm{FSTI}=4 \%$

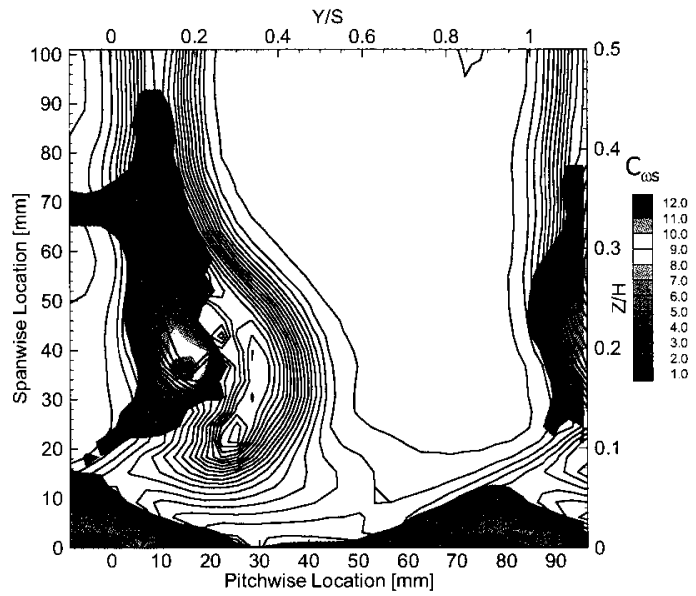

Figure G.9: PAKDF - Line Contours of Total Pressure Coefficient Superimposed on Flood Contours of Positive Vorticity at $+5^{\circ}$ Incidence at FSTI $=1.5 \%$ and $4.0 \%$ 

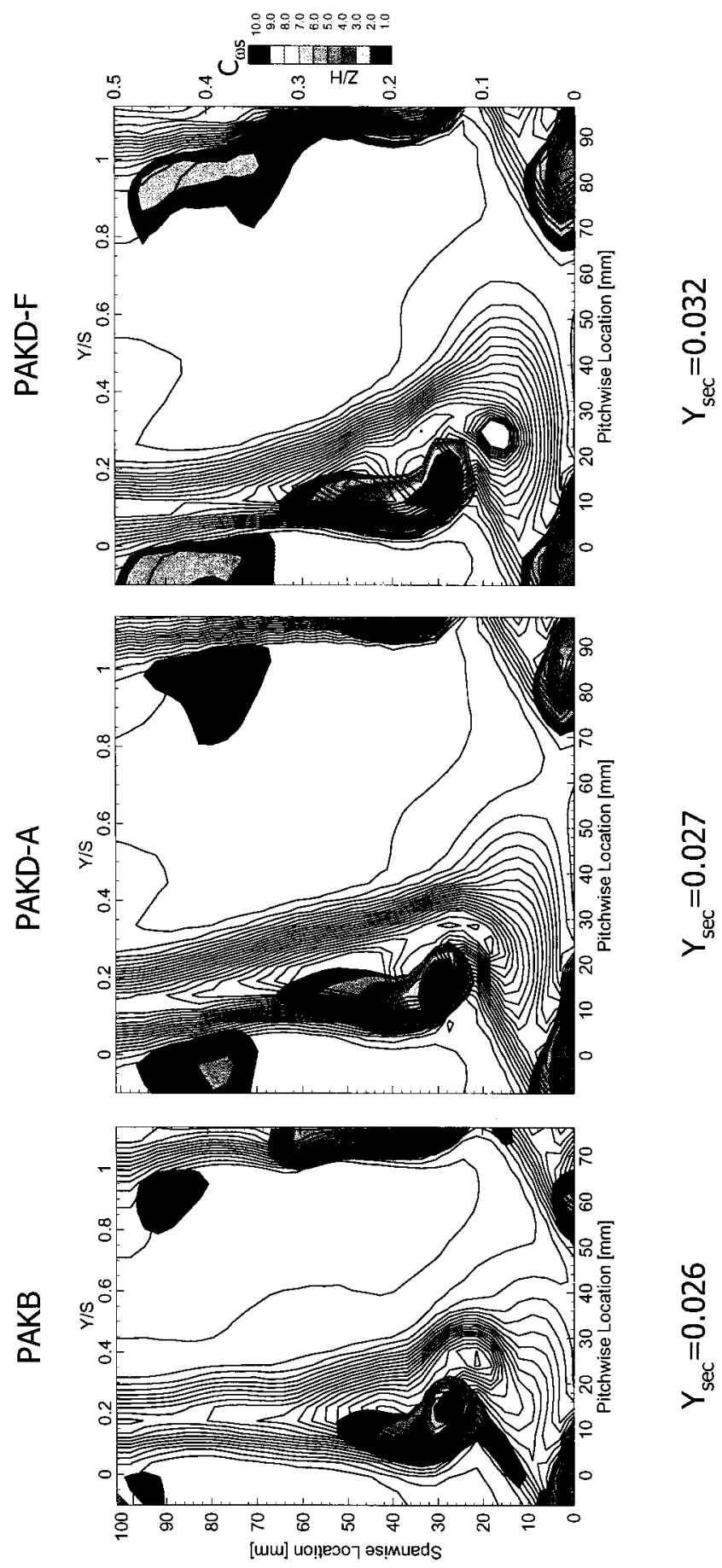

Figure G.10: Line Contours of Total Pressure Coefficient Superimposed on Flood Contours of Positive Vorticity at $-5^{\circ}$ Incidence at FSTI $=1.5 \%$ 

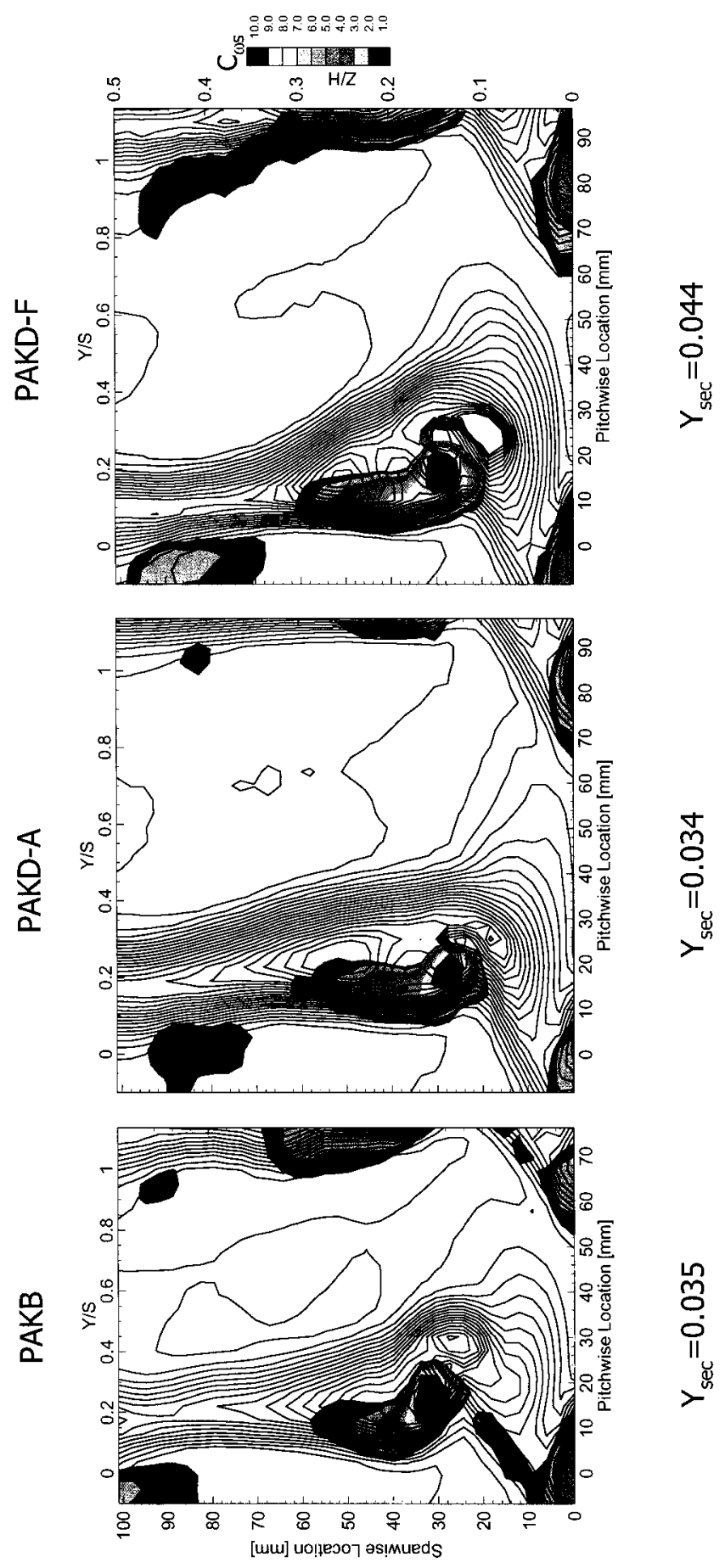

Figure G.11: Line Contours of Total Pressure Coefficient Superimposed on Flood Contours of Positive Vorticity at $0^{\circ}$ Incidence at FSTI $=1.5 \%$ 

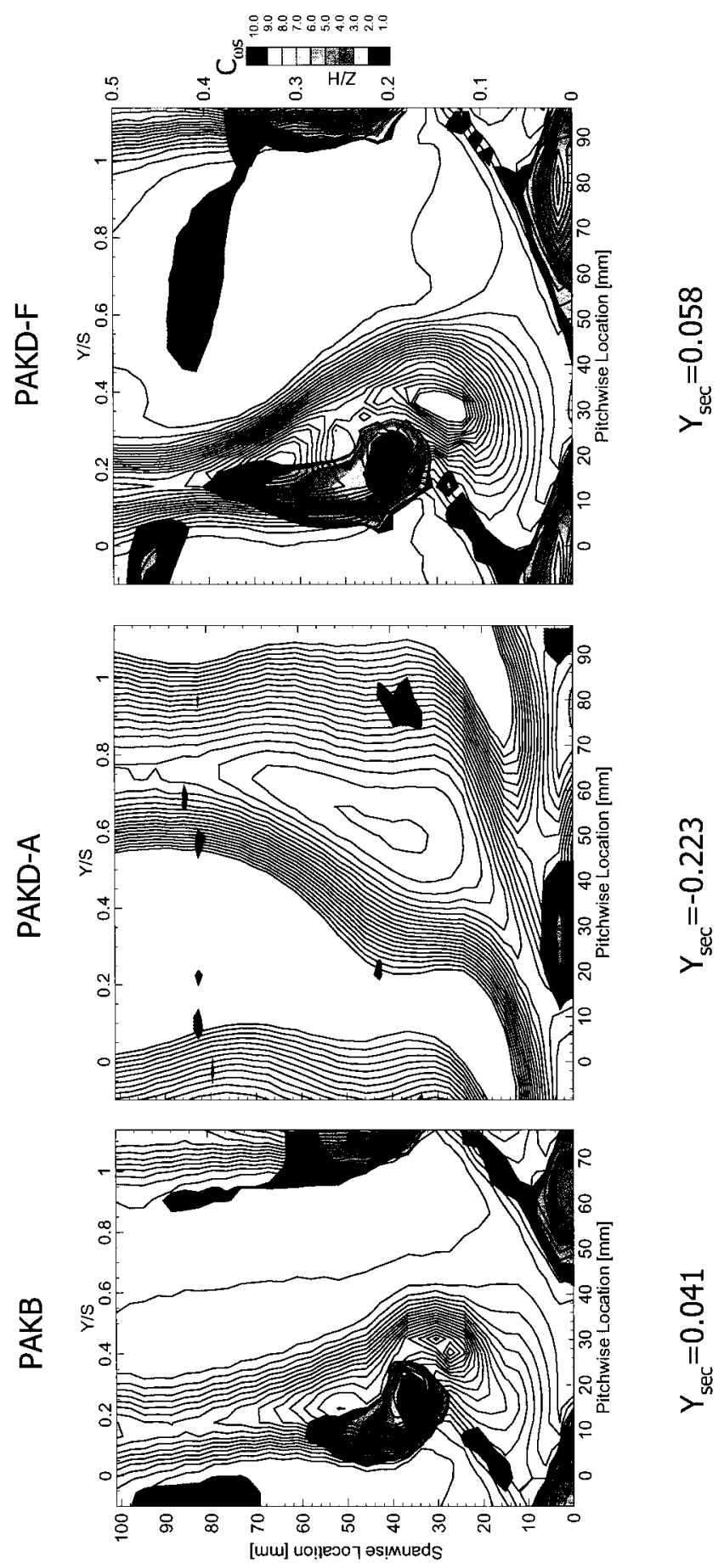

Figure G.12: Line Contours of Total Pressure Coefficient Superimposed on Flood Contours of Positive Vorticity at $+5^{\circ}$ Incidence at FSTI $=1.5 \%$ 

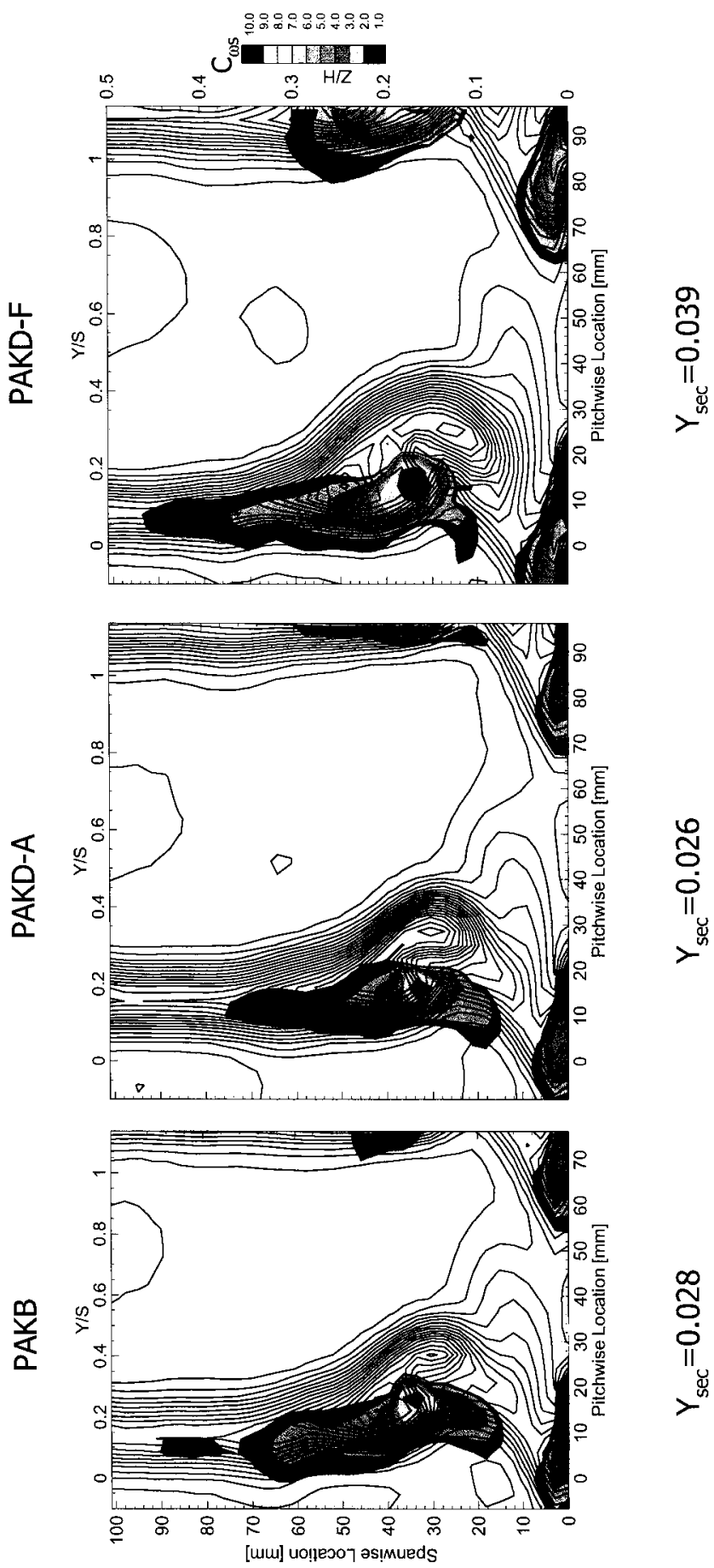

Figure G.13: Line Contours of Total Pressure Coefficient Superimposed on Flood Contours of Positive Vorticity at $0^{\circ}$ Incidence at FSTI $=4.0 \%$ 

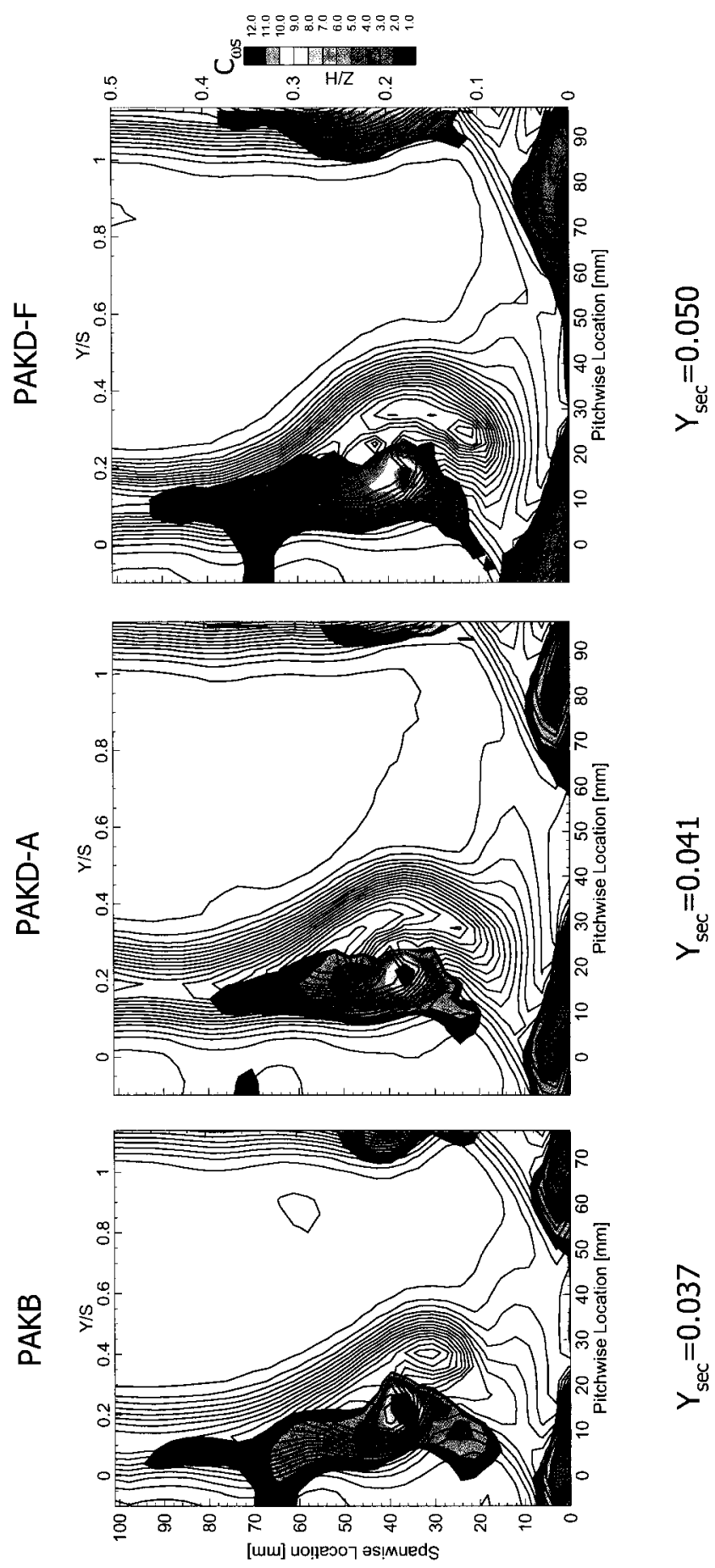

Figure G.14: Line Contours of Total Pressure Coefficient Superimposed on Flood Contours of Positive Vorticity at $+5^{\circ}$ Incidence at FSTI $=4.0 \%$ 


\section{Appendix $\mathrm{H}$}

\section{Negative Vorticity}

Negative vorticity superimposed with total pressure coefficient line contours is presented in this appendix. As in the case of positive vorticity, the definition of streamwise vorticity was given in Chapter 3 and discussed in Chapters 4 and 5, respectively. The negative vorticity is rotating in clockwise direction and is associated with the passage vortex. It is located close to the endwall.

The appendix is organized exactly in the same way as the previous ones. It begins with comparison of negative non-dimensional vorticity, $C_{\omega}$, contours for the PAKB blade at three incidences examined $\left(-5^{\circ}, 0^{\circ},+5^{\circ}\right)$ and FSTI of $1.5 \%$. It continues with comparison of PAKB contours for two FSTIs (1.5\% and 4.0\%) at design and positive off-design incidence. The same scheme is followed for the remaining two blades, PAKD-A, and PAKD-F. Lastly all three blades are compared at all incidences and FSTIs examined. 

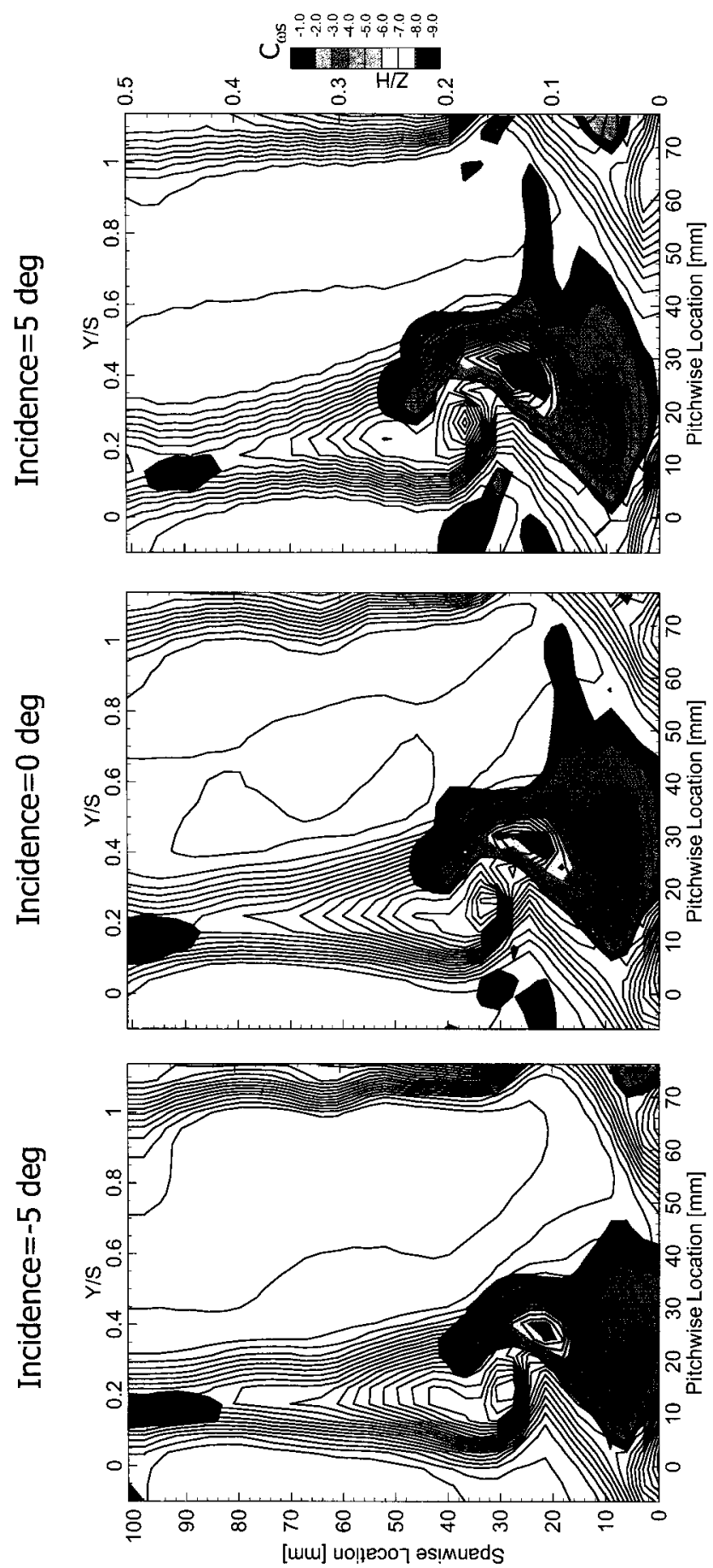

Figure H.1: PAKB - Line Contours of Total Pressure Coefficient Superimposed on Flood Contours of Negative Vorticity at Design and Off-Design Conditions at $\mathrm{FSTI}=1.5 \%$ 

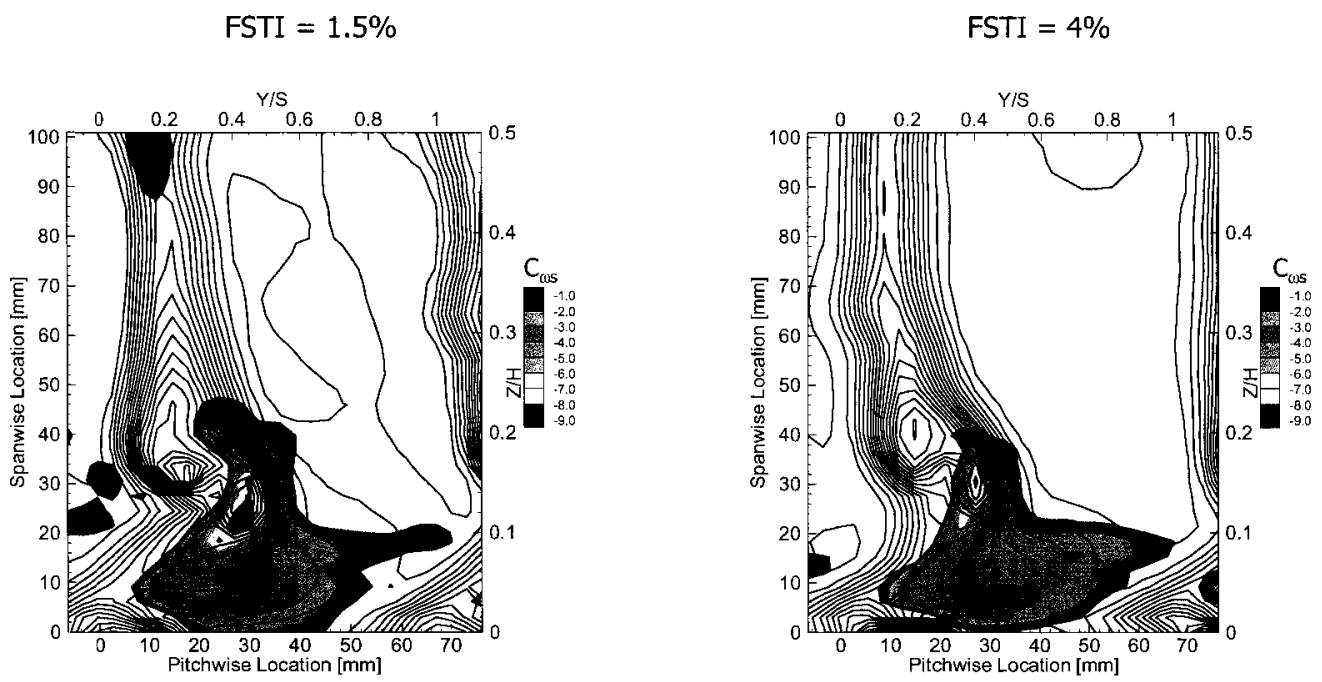

Figure H.2: PAKB - Line Contours of Total Pressure Coefficient Superimposed on Flood Contours of Negative Vorticity at Design Incidence at FSTI $=1.5 \%$ and $4.0 \%$

$\mathrm{FSTI}=1.5 \%$

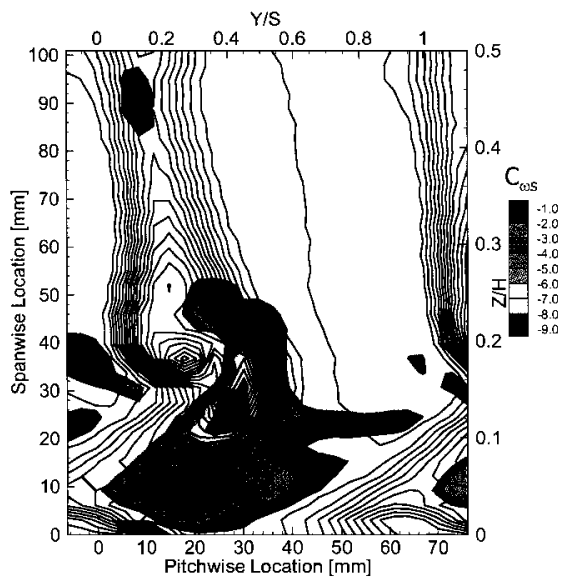

$\mathrm{FSTI}=4 \%$

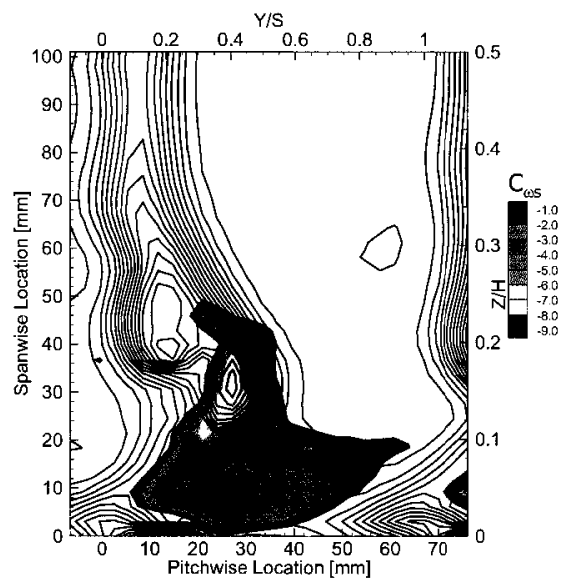

Figure H.3: PAKB - Line Contours of Total Pressure Coefficient Superimposed on Flood Contours of Negative Vorticity at $+5^{\circ}$ Incidence at FSTI $=1.5 \%$ and $4.0 \%$ 

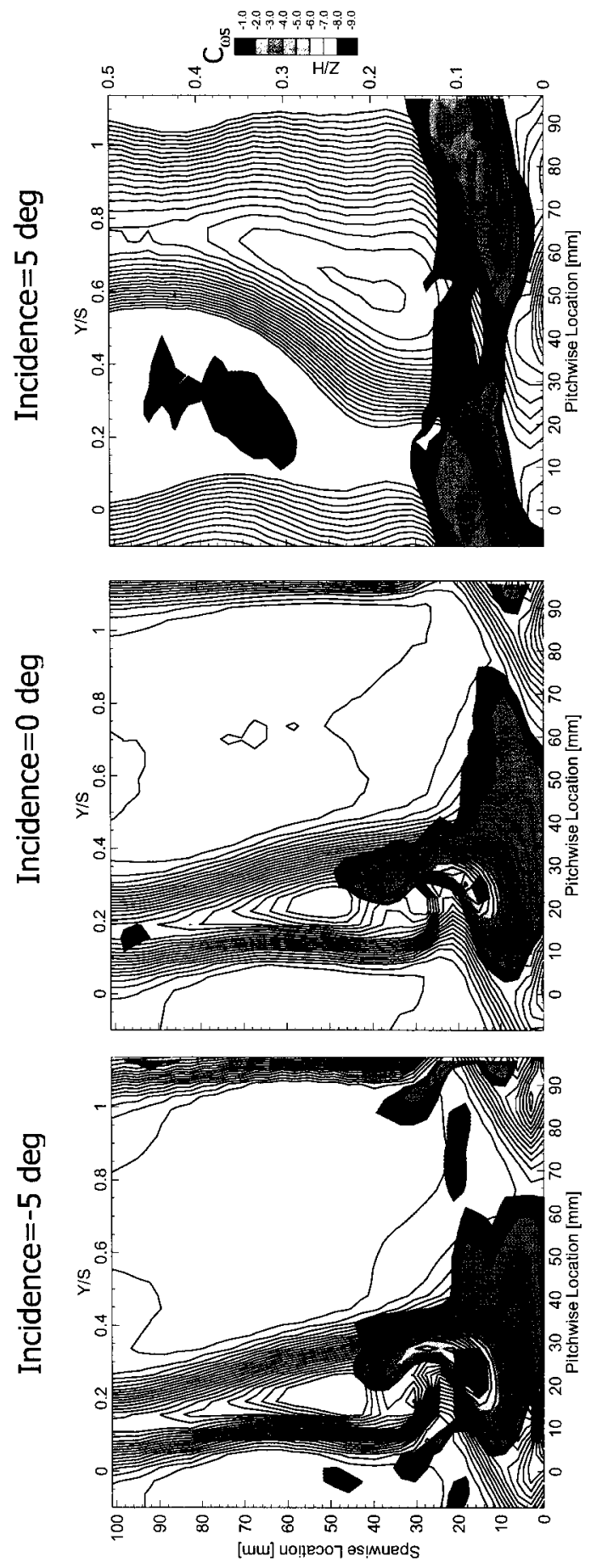

Figure H.4: PAKDA - Line Contours of Total Pressure Coefficient Superimposed on Flood Contours of Negative Vorticity at Design and Off-Design Conditions at $\mathrm{FSTI}=1.5 \%$ 

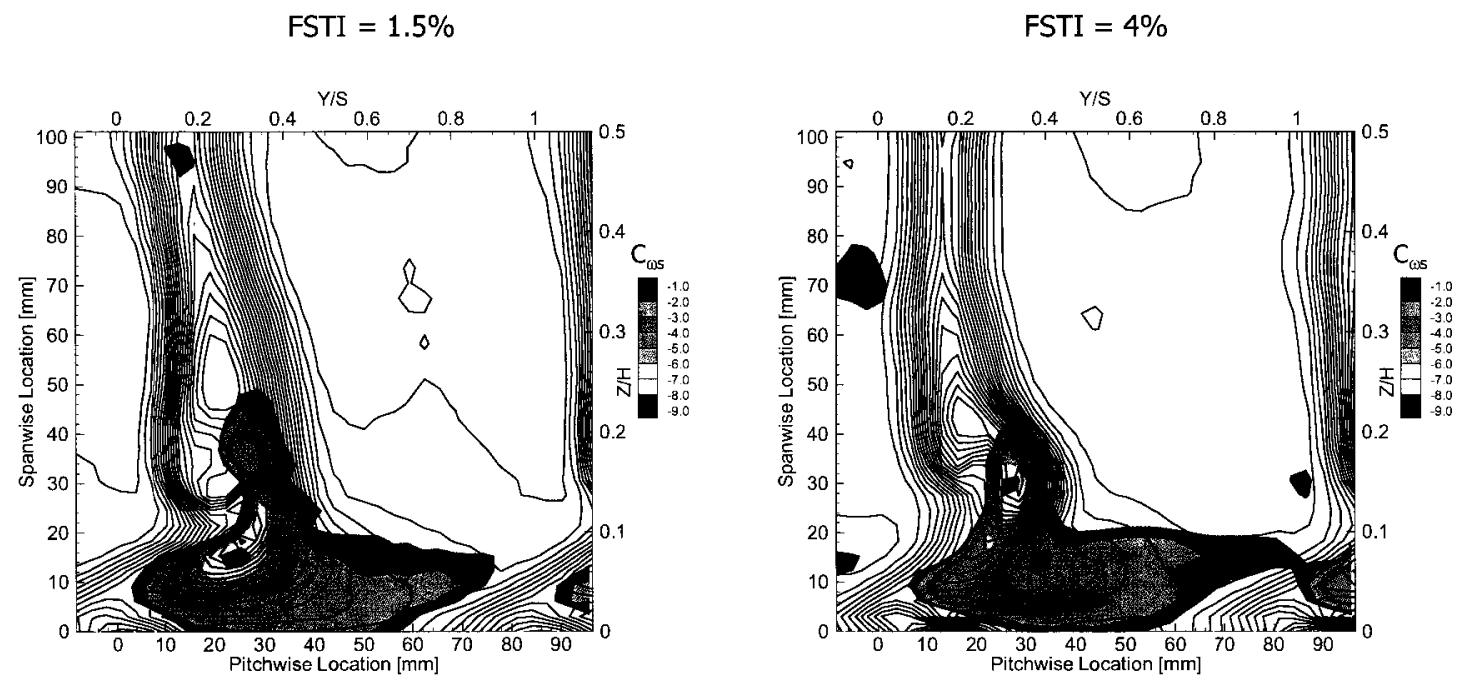

Figure H.5: PAKDA - Line Contours of Total Pressure Coefficient Superimposed on Flood Contours of Negative Vorticity at Design Incidence at FSTI $=1.5 \%$ and $4.0 \%$

$$
\mathrm{FSTI}=1.5 \%
$$

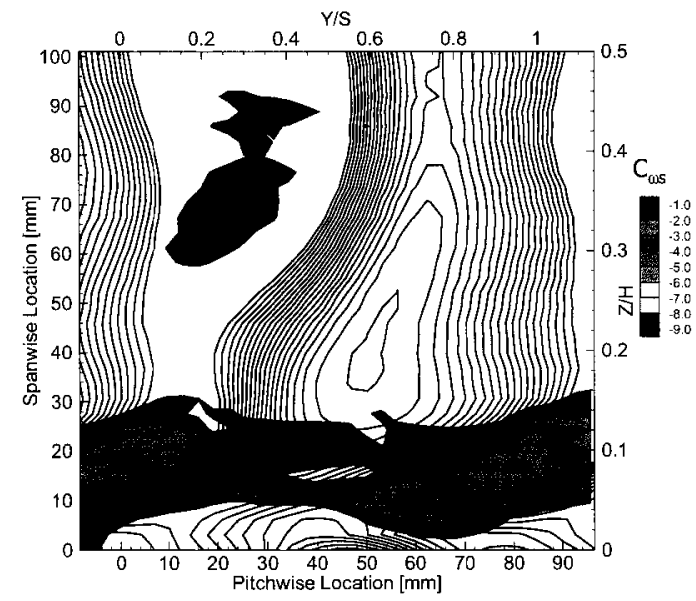

$\mathrm{FSTI}=4 \%$

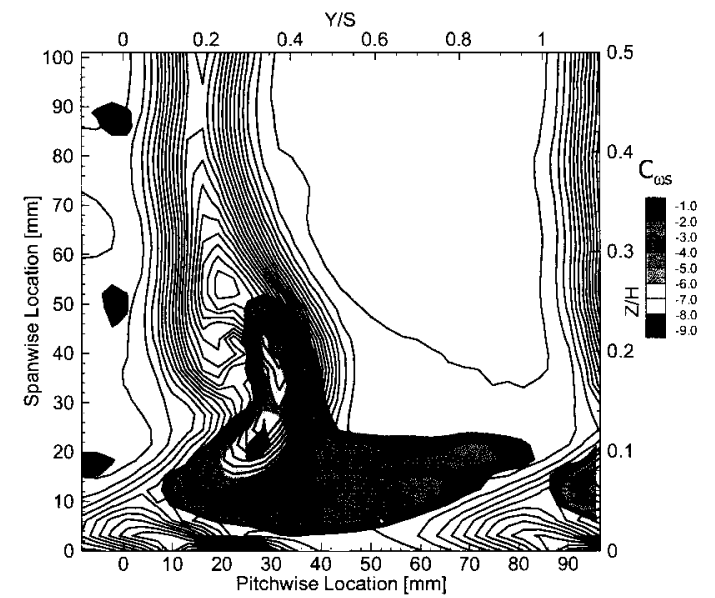

Figure H.6: PAKDA - Line Contours of Total Pressure Coefficient Superimposed on Flood Contours of Negative Vorticity at $+5^{\circ}$ Incidence at FSTI $=1.5 \%$ and $4.0 \%$ 

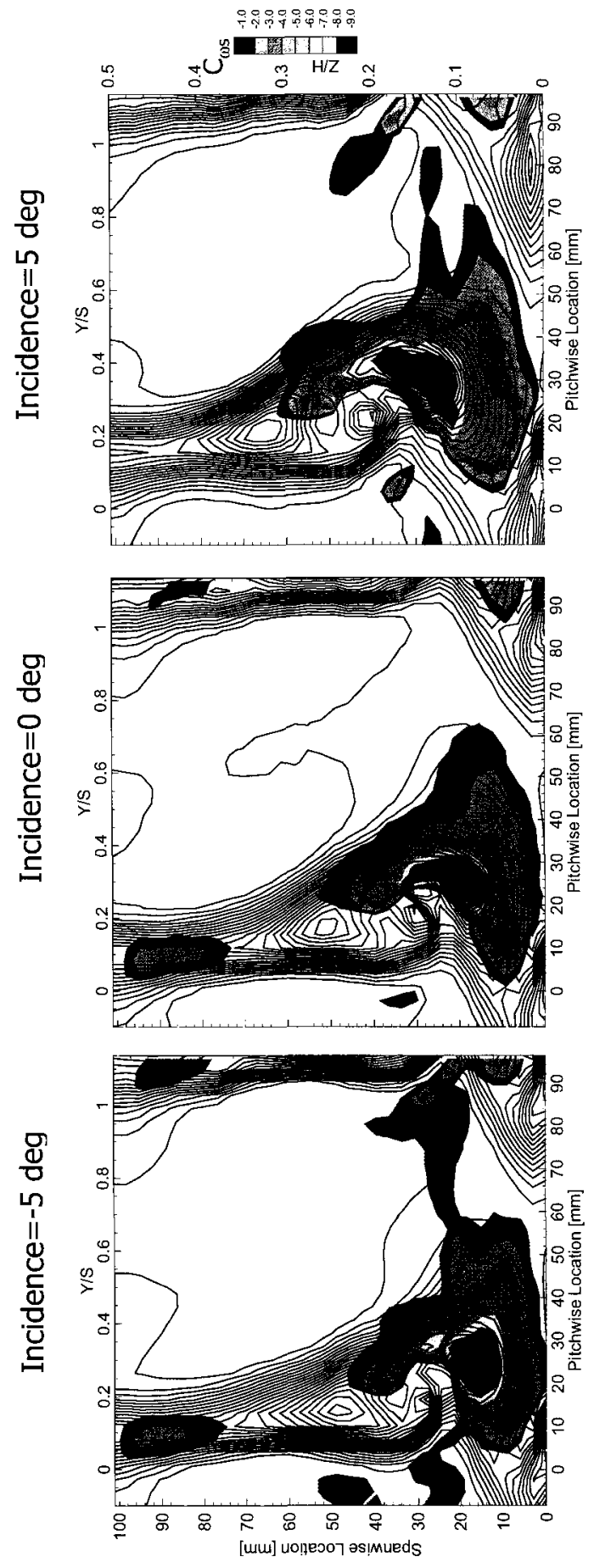

Figure H.7: PAKDF - Line Contours of Total Pressure Coefficient Superimposed on Flood Contours of Negative Vorticity at Design and Off-Design Conditions at $\mathrm{FSTI}=1.5 \%$ 
$\mathrm{FSTI}=1.5 \%$

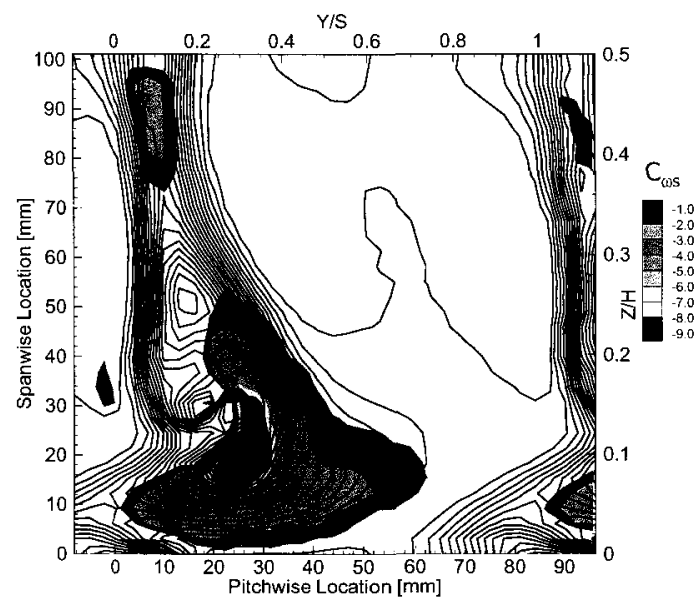

$\mathrm{FSTI}=4 \%$

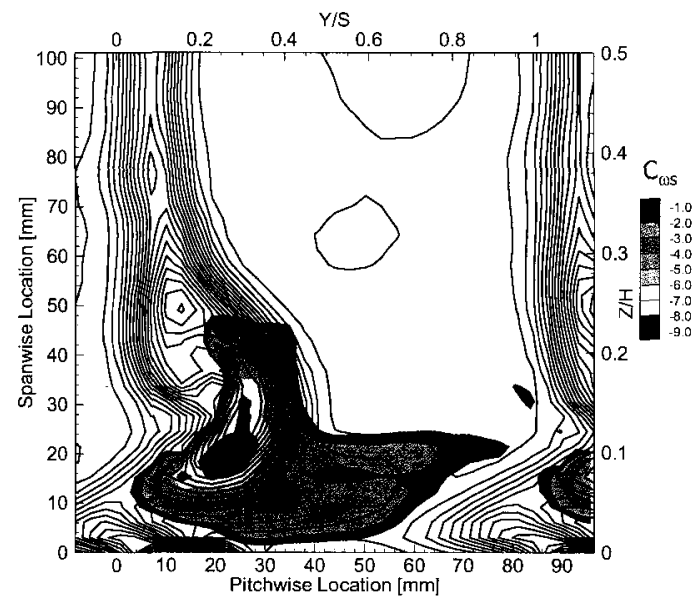

Figure H.8: PAKDF - Line Contours of Total Pressure Coefficient Superimposed on Flood Contours of Negative Vorticity at Design Incidence at FSTI $=1.5 \%$ and $4.0 \%$

$$
\text { FSTI }=1.5 \%
$$

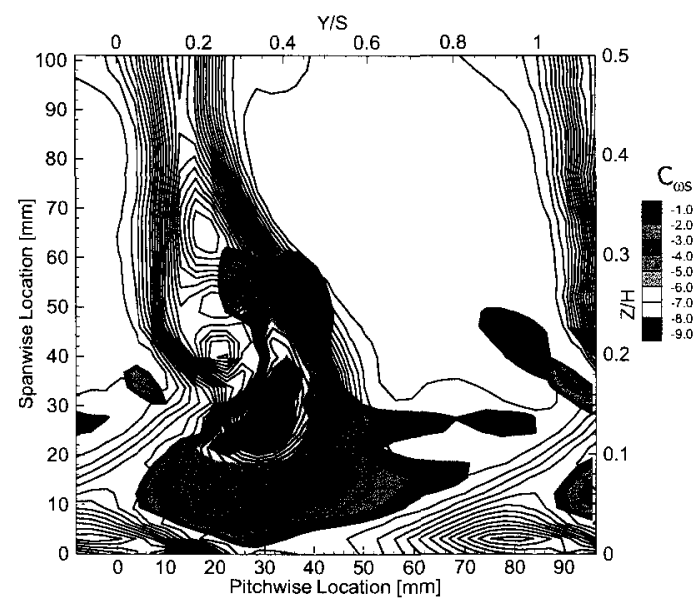

$\mathrm{FSTI}=4 \%$

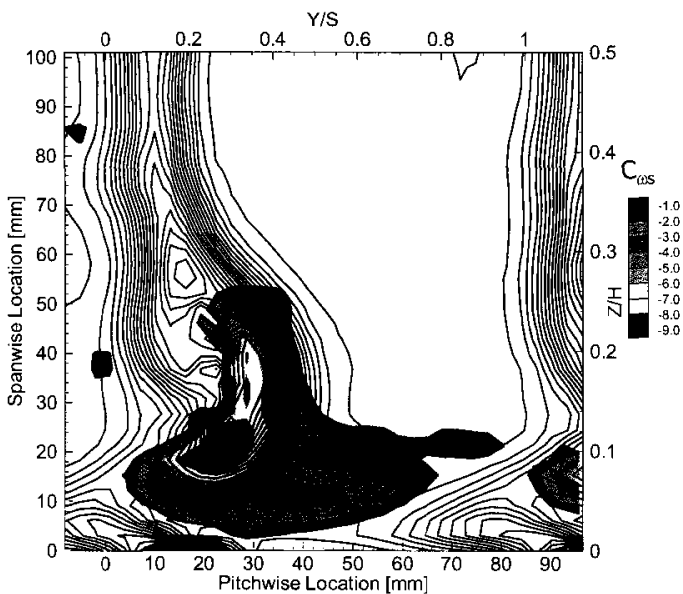

Figure H.9: PAKDF - Line Contours of Total Pressure Coefficient Superimposed on Flood Contours of Negative Vorticity at $+5^{\circ}$ Incidence at FSTI $=1.5 \%$ and $4.0 \%$ 

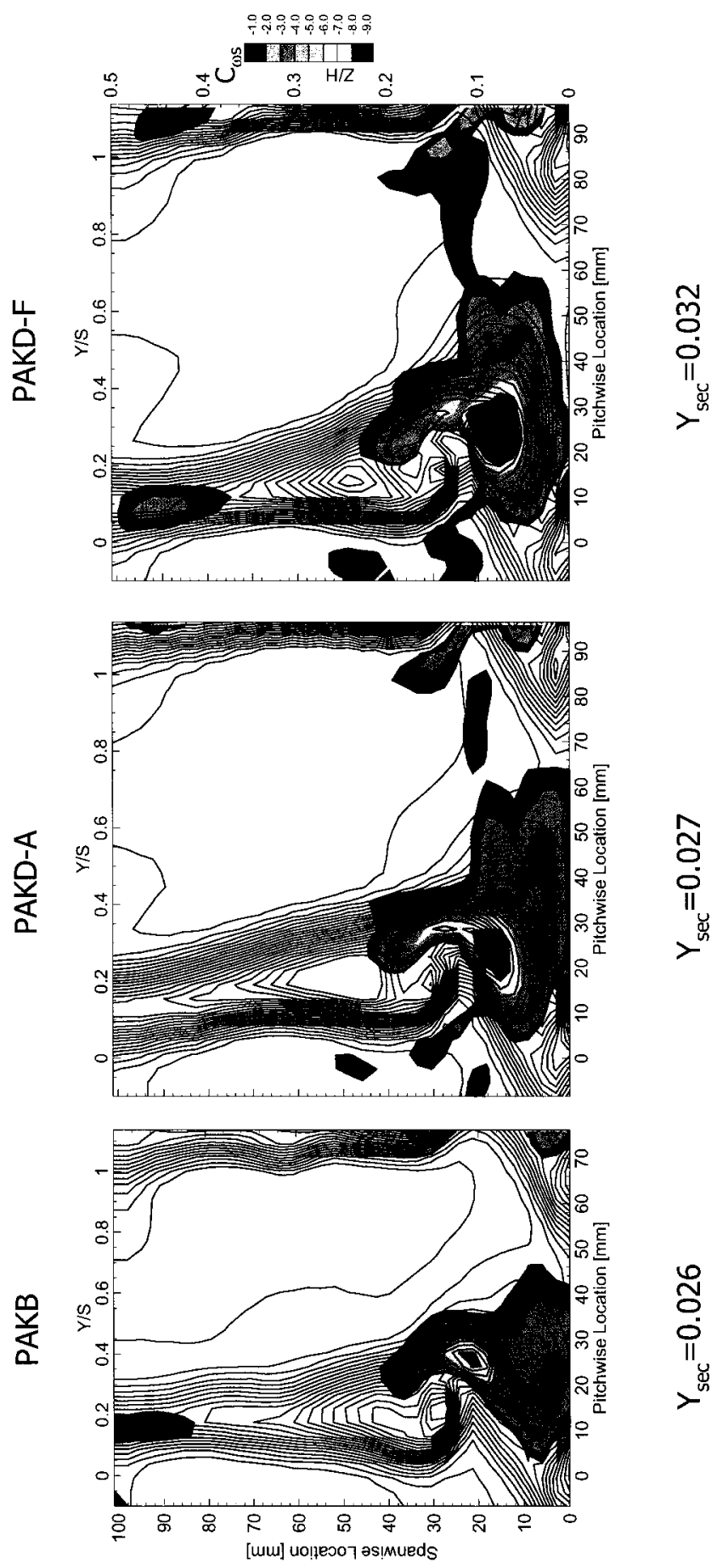

Figure H.10: Line Contours of Total Pressure Coefficient Superimposed on Flood Contours of Negative Vorticity at $-5^{\circ}$ Incidence at FSTI $=1.5 \%$ 

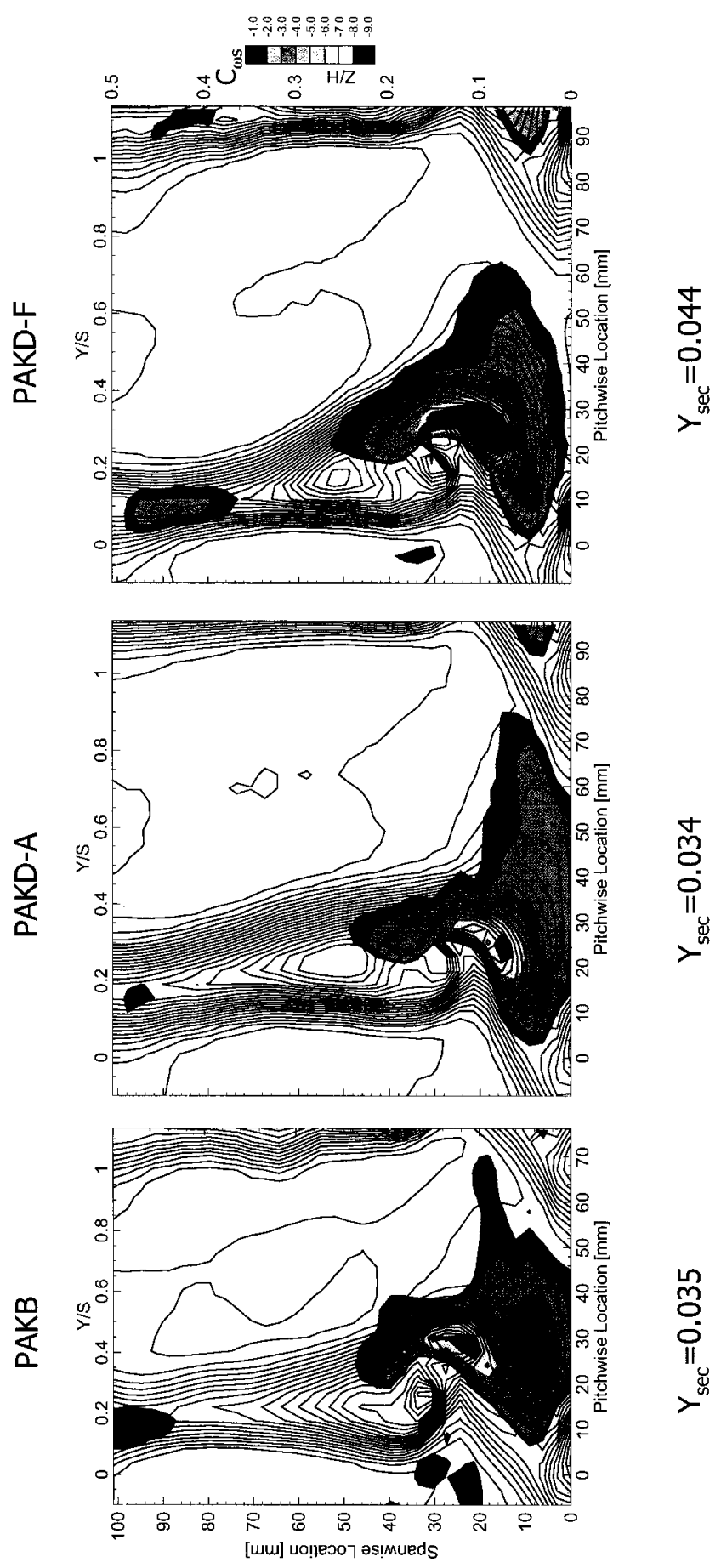

Figure H.11: Line Contours of Total Pressure Coefficient Superimposed on Flood Contours of Negative Vorticity at $0^{\circ}$ Incidence at FSTI $=1.5 \%$ 

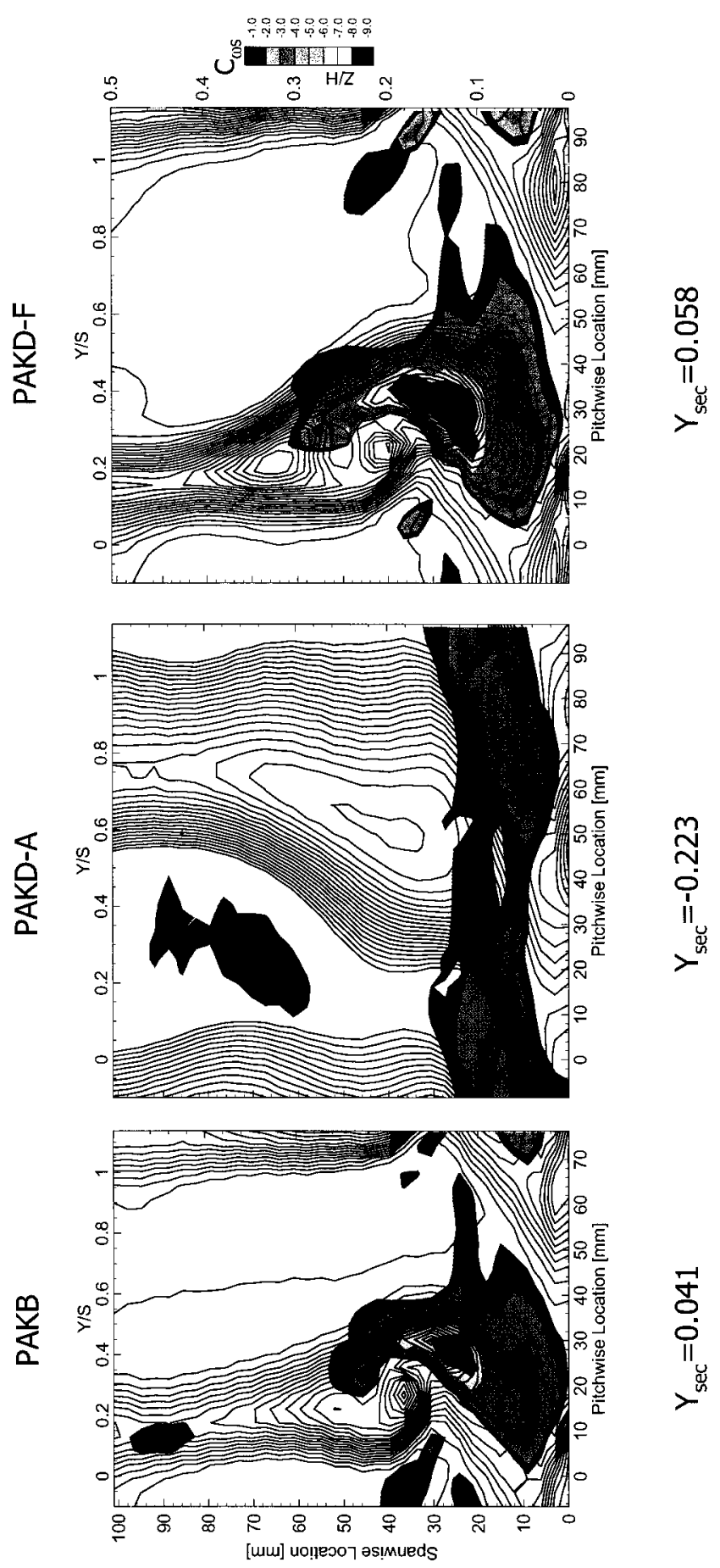

Figure H.12: Line Contours of Total Pressure Coefficient Superimposed on Flood Contours of Negative Vorticity at $+5^{\circ}$ Incidence at FSTI $=1.5 \%$ 


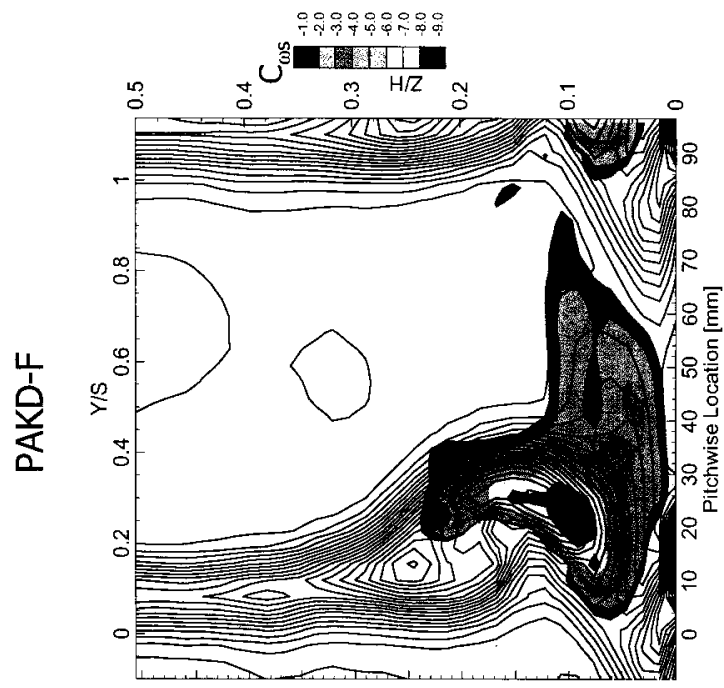

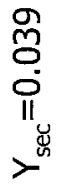

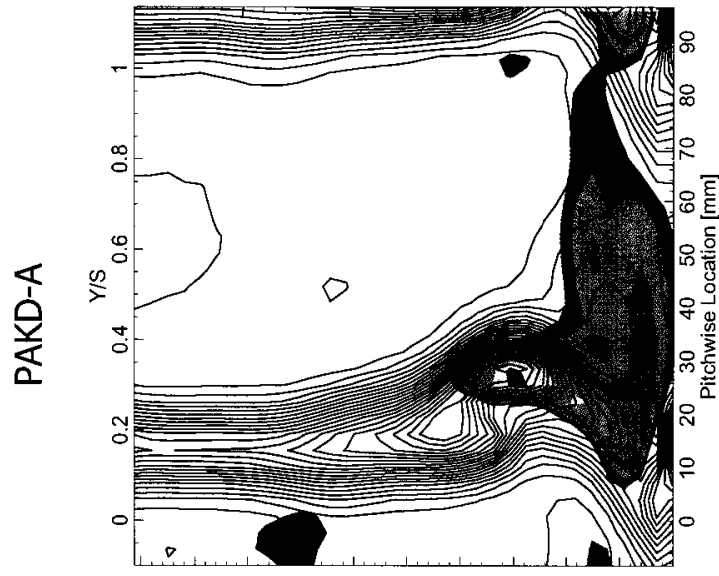

0
0
0
$I_{u}$
un
$\nu$

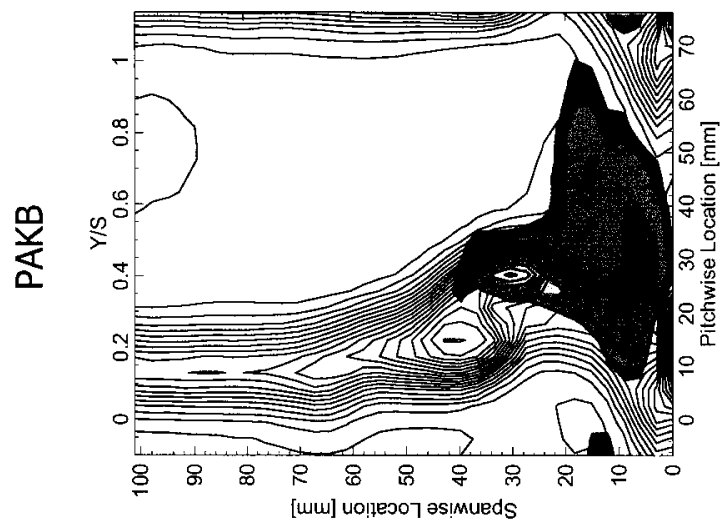

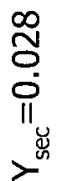

Figure H.13: Line Contours of Total Pressure Coefficient Superimposed on Flood Contours of Negative Vorticity at $0^{\circ}$ Incidence at FSTI $=4.0 \%$ 


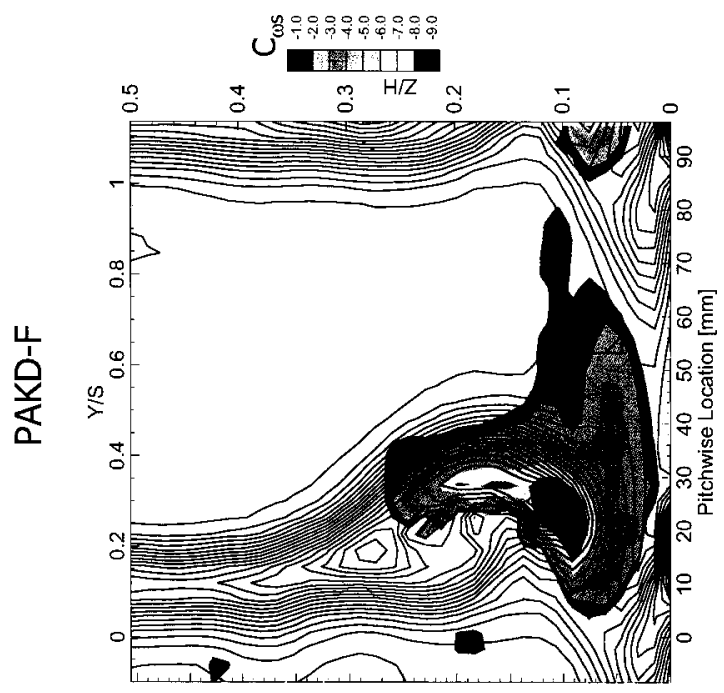

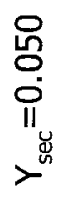

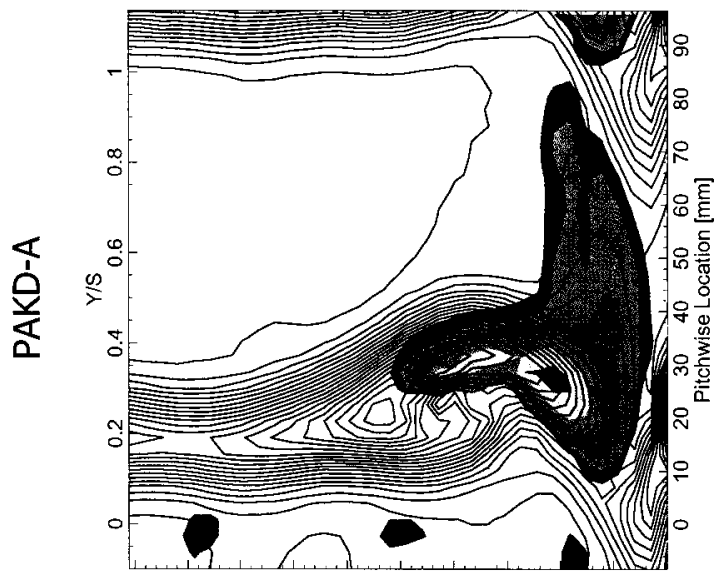

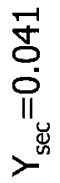

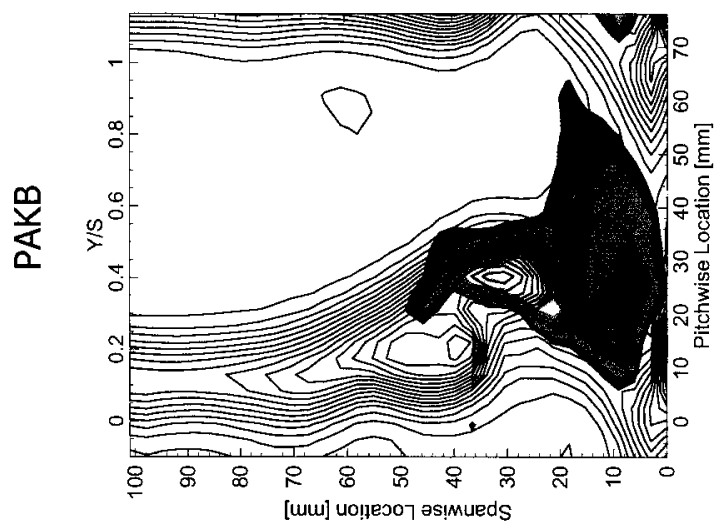

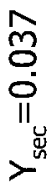

Figure H.14: Line Contours of Total Pressure Coefficient Superimposed on Flood Contours of Negative Vorticity at $+5^{\circ}$ Incidence at FSTI $=4.0 \%$ 


\section{Appendix I}

\section{Axial Velocity Distribution}

The axial velocity ratio distribution $(A V R)$, contours for all three blades at design and off-design incidences and both FSTIs are presented in this appendix. AVR was defined in equation 3.19 in Chapter 3 and discussed in Chapters 4 and 5.

The appendix format is the same as in appendices F, H, and, H. First the comparison of $A V R$ contours for the PAKB blade at all examined incidences and FSTI of $1.5 \%$ is shown. Following that, a comparison of PAKB $A V R$ contours for two FSTIs (1.5\% and 4.0\%) at design and positive off-design incidence is presented. The same layout is applied for PAKD-A and PAKD-F. Finally all three blades are compared at all incidences and FSTIs examined. 

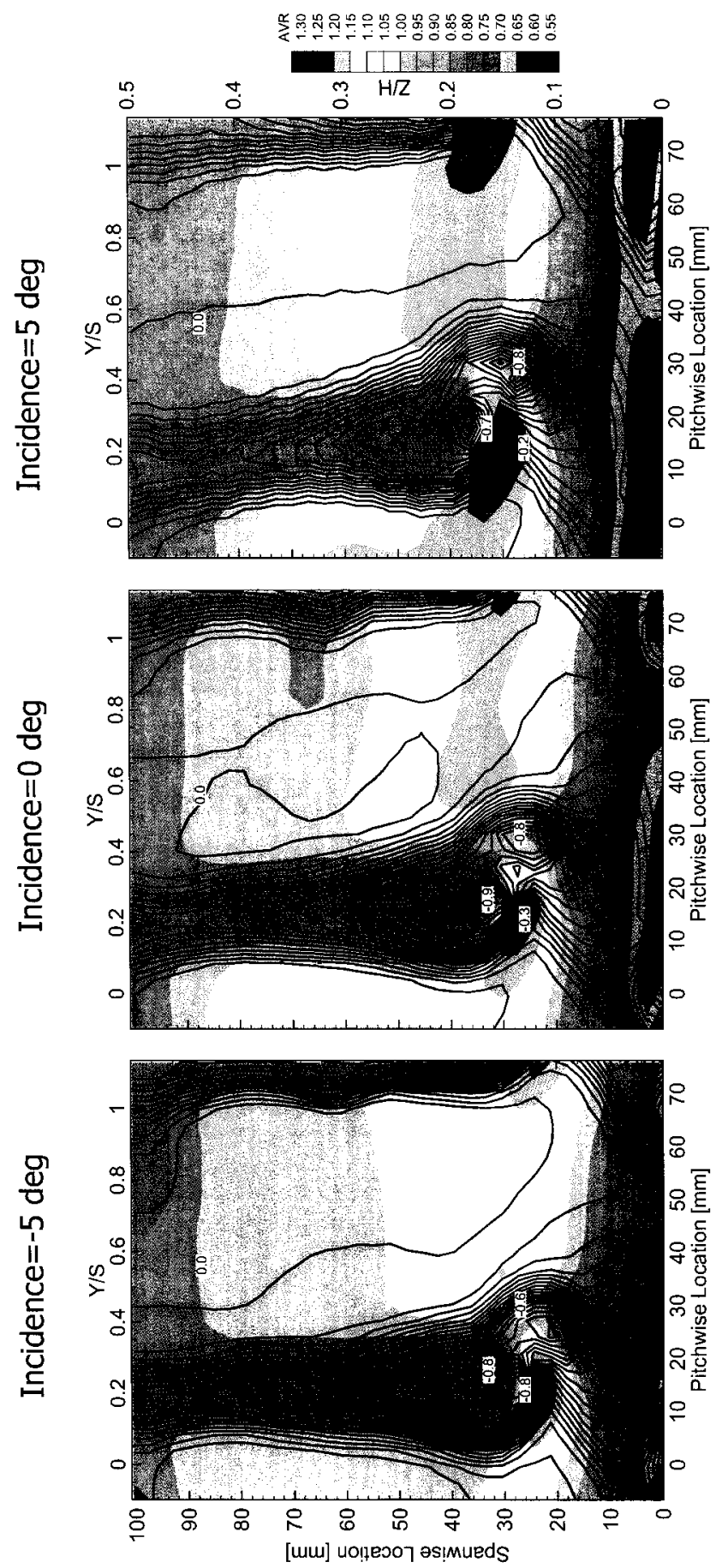

Figure I.1: PAKB - Line Contours of Total Pressure Coefficient Superimposed on Flood Contours of AVR at Design and Off-Design Conditions at FSTI=1.5\% 

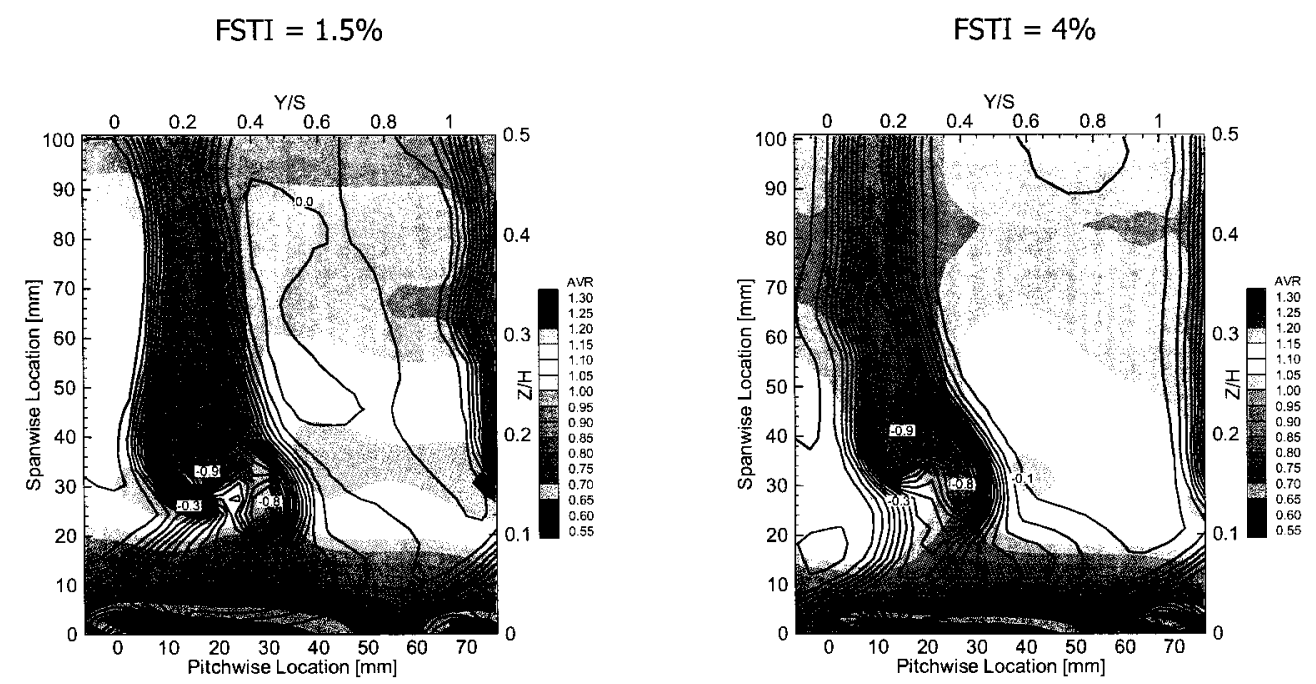

Figure I.2: PAKB - Line Contours of Total Pressure Coefficient Superimposed on Flood Contours of AVR at Design Incidence at FSTI $=1.5 \%$ and $4.0 \%$
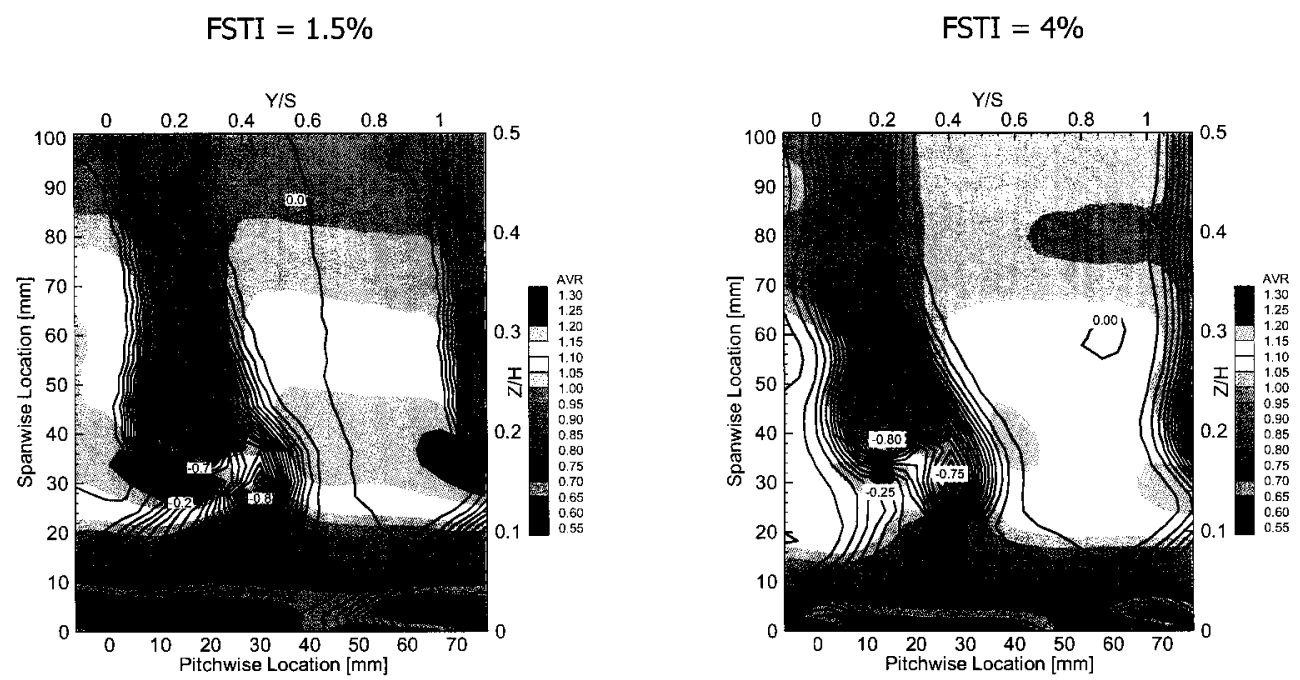

Figure I.3: PAKB - Line Contours of Total Pressure Coefficient Superimposed on Flood Contours of AVR at $+5^{\circ}$ Incidence at FSTI $=1.5 \%$ and $4.0 \%$ 

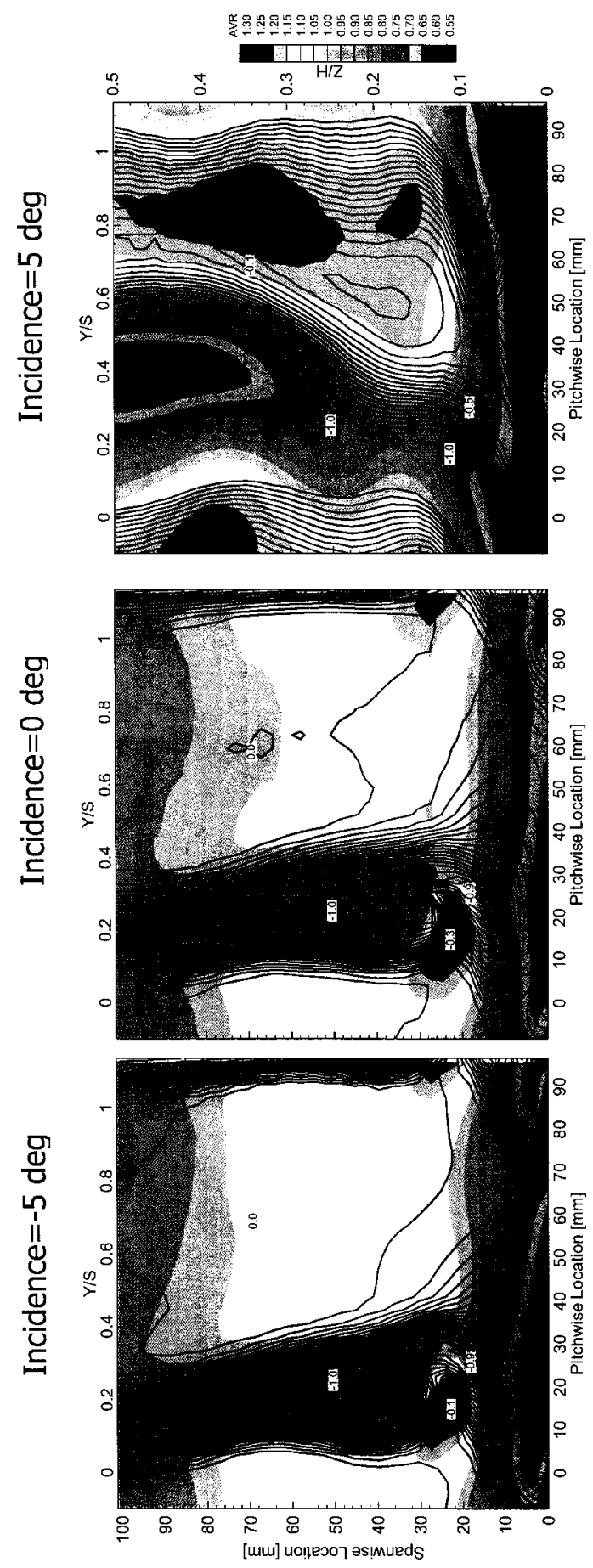

Figure I.4: PAKDA - Line Contours of Total Pressure Coefficient Superimposed on Flood Contours of AVR at Design and Off-Design Conditions at FSTI=1.5\% 

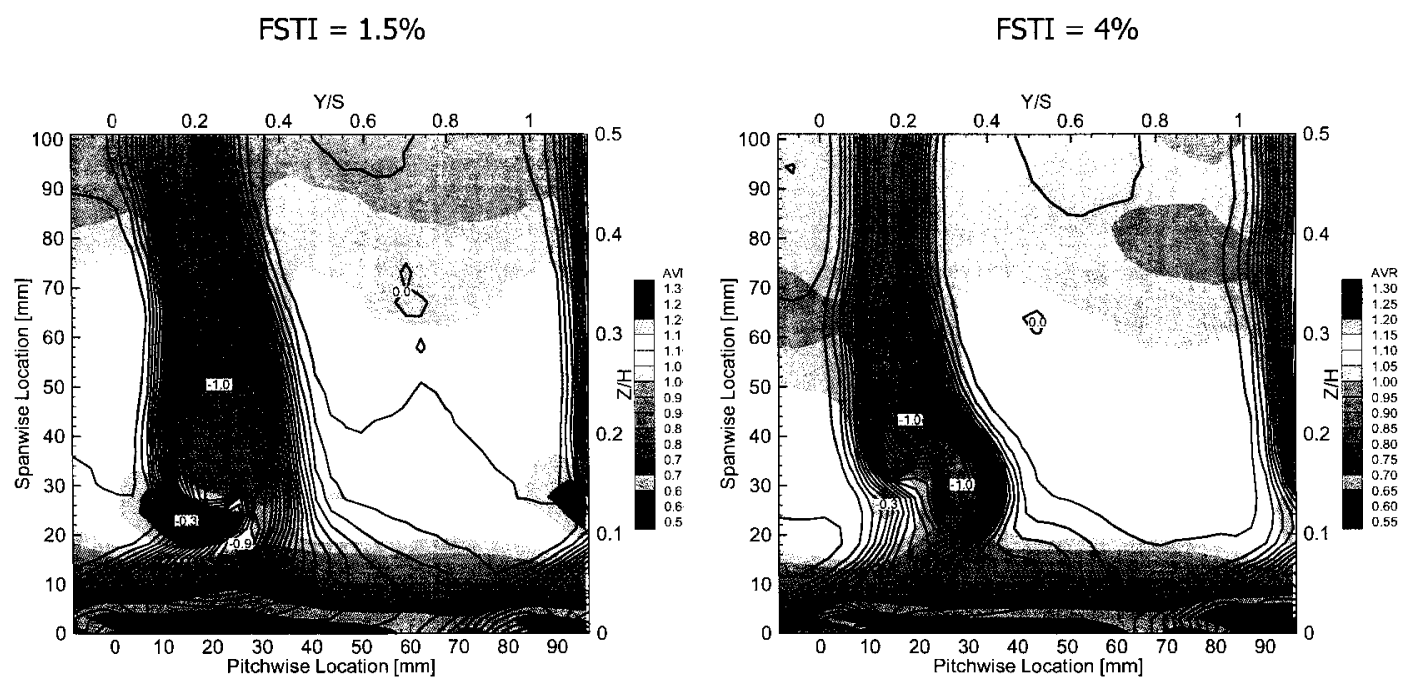

Figure I.5: PAKDA - Line Contours of Total Pressure Coefficient Superimposed on Flood Contours of AVR at Design Incidence at FSTI $=1.5 \%$ and $4.0 \%$

$$
\mathrm{FSTI}=1.5 \%
$$

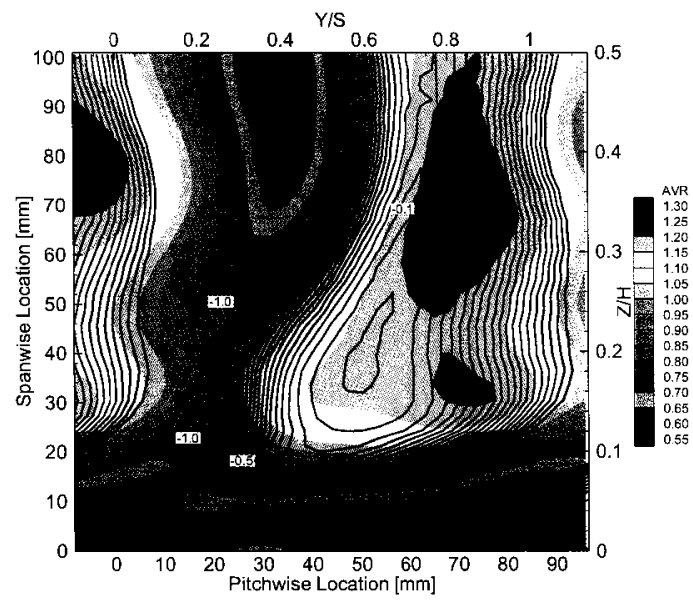

$\mathrm{FSTI}=4 \%$

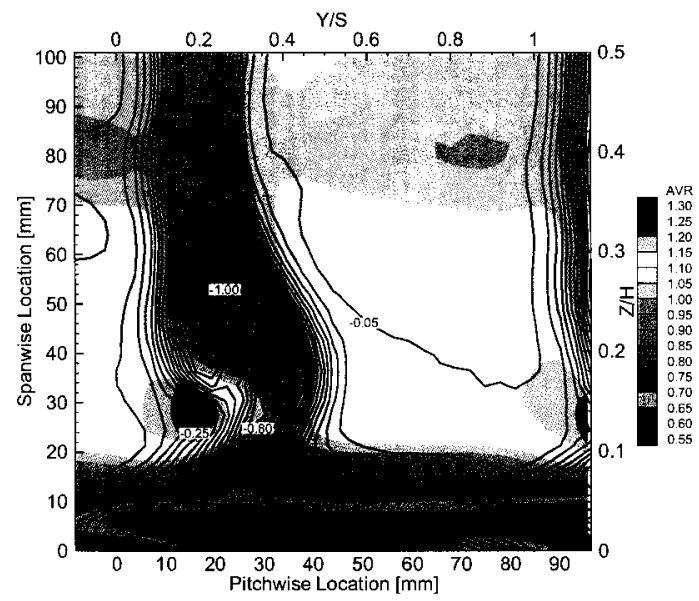

Figure I.6: PAKDA - Line Contours of Total Pressure Coefficient Superimposed on Flood Contours of AVR at $+5^{\circ}$ Incidence at FSTI $=1.5 \%$ and $4.0 \%$ 

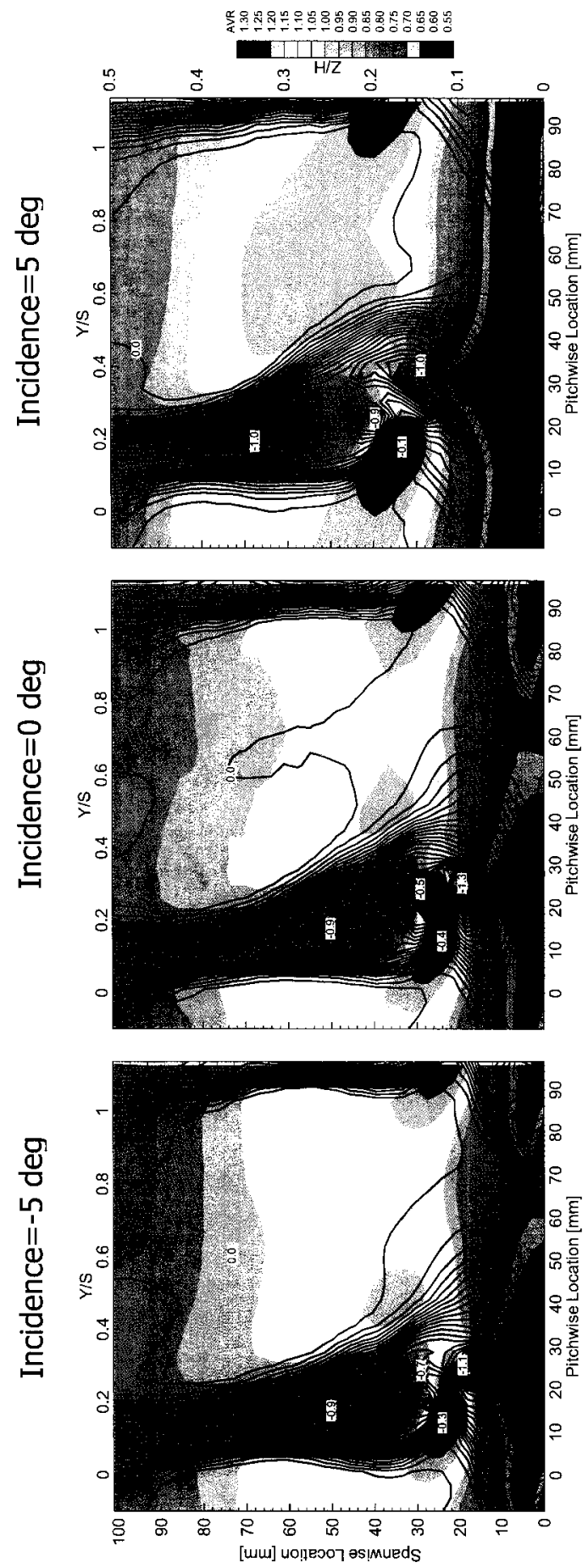

Figure I.7: PAKDF - Line Contours of Total Pressure Coefficient Superimposed on Flood Contours of AVR at Design and Off-Design Conditions at FSTI $=1.5 \%$ 

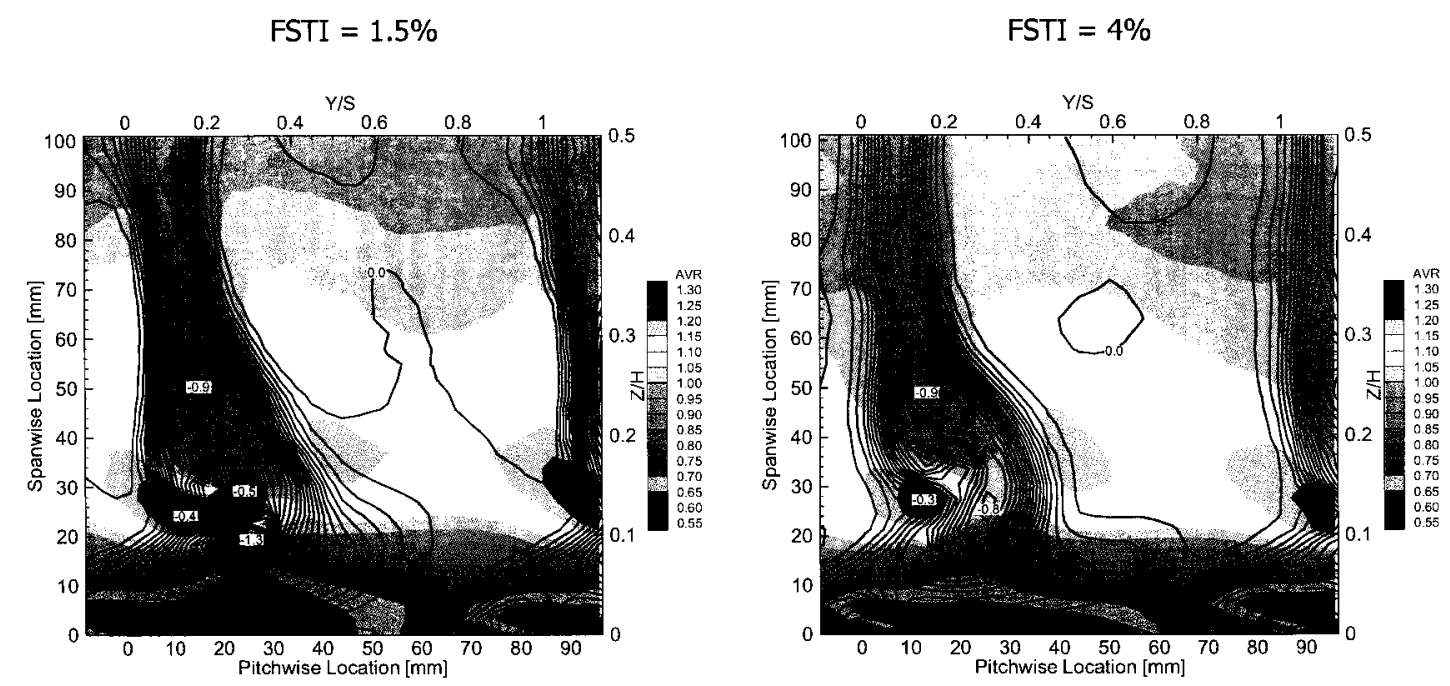

Figure I.8: PAKDF - Line Contours of Total Pressure Coefficient Superimposed on Flood Contours of AVR at Design Incidence at FSTI $=1.5 \%$ and $4.0 \%$
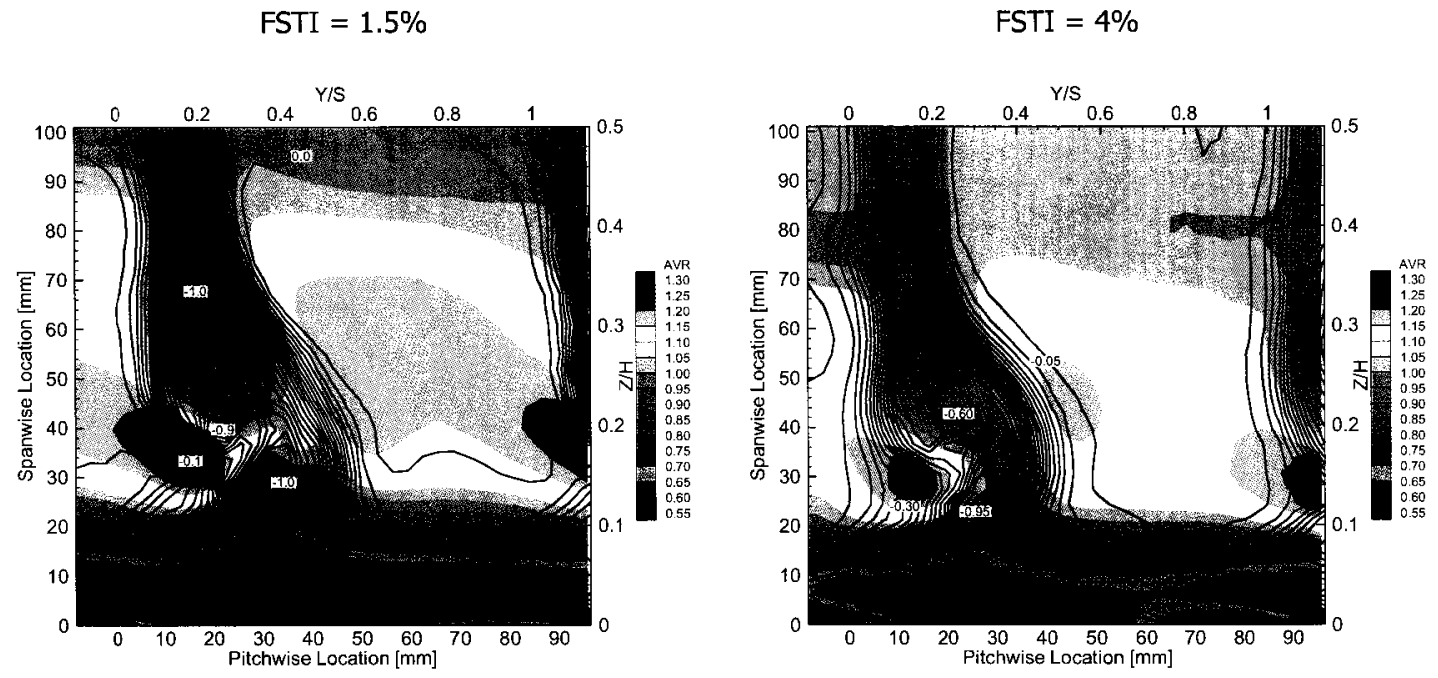

Figure 1.9: PAKDF - Line Contours of Total Pressure Coefficient Superimposed on Flood Contours of AVR at $+5^{\circ}$ Incidence at FSTI $=1.5 \%$ and $4.0 \%$ 

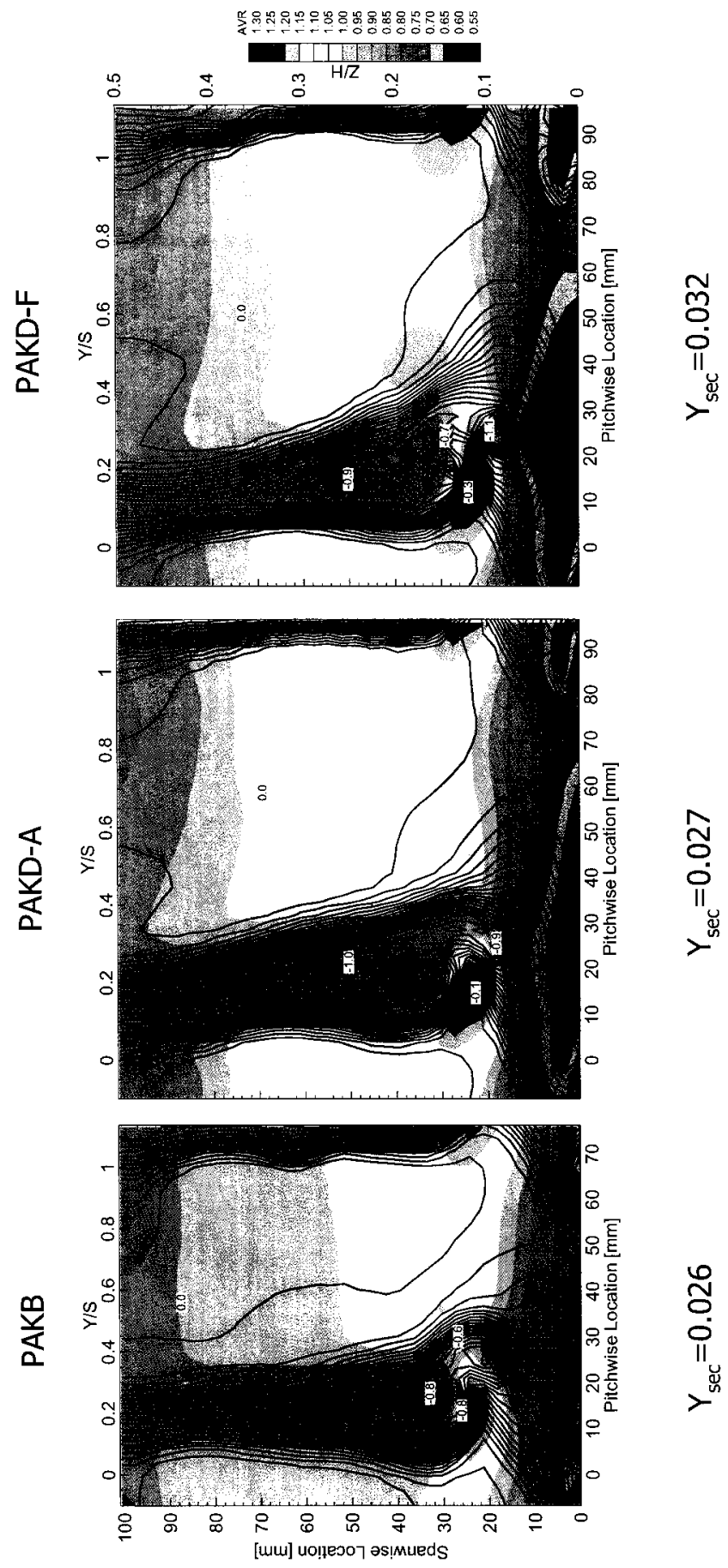

Figure I.10: Line Contours of Total Pressure Coefficient Superimposed on Flood Contours of AVR at $-5^{\circ}$ Incidence at FSTI $=1.5 \%$ 

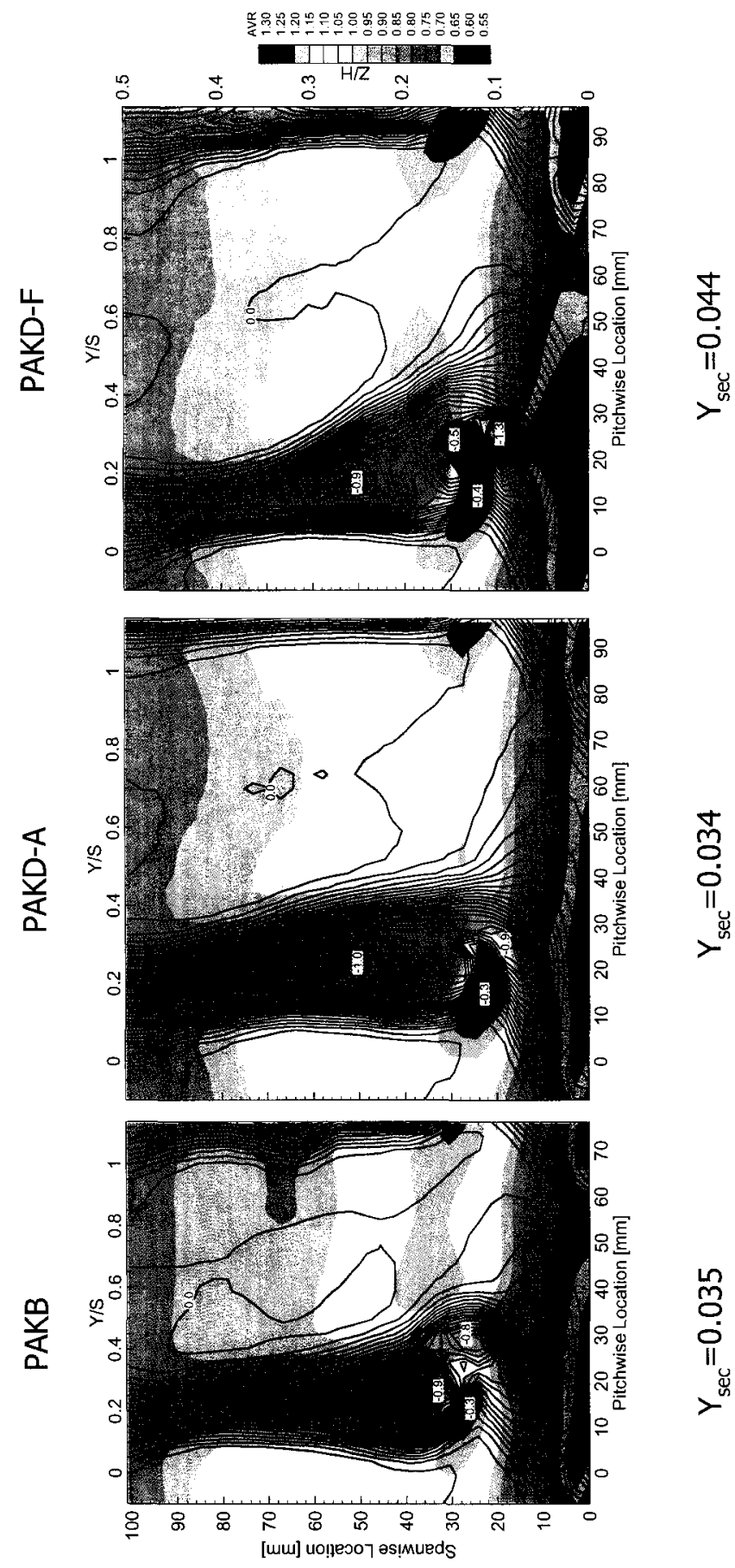

Figure 1.11: Line Contours of Total Pressure Coefficient Superimposed on Flood Contours of AVR at $0^{\circ}$ Incidence at FSTI $=1.5 \%$ 

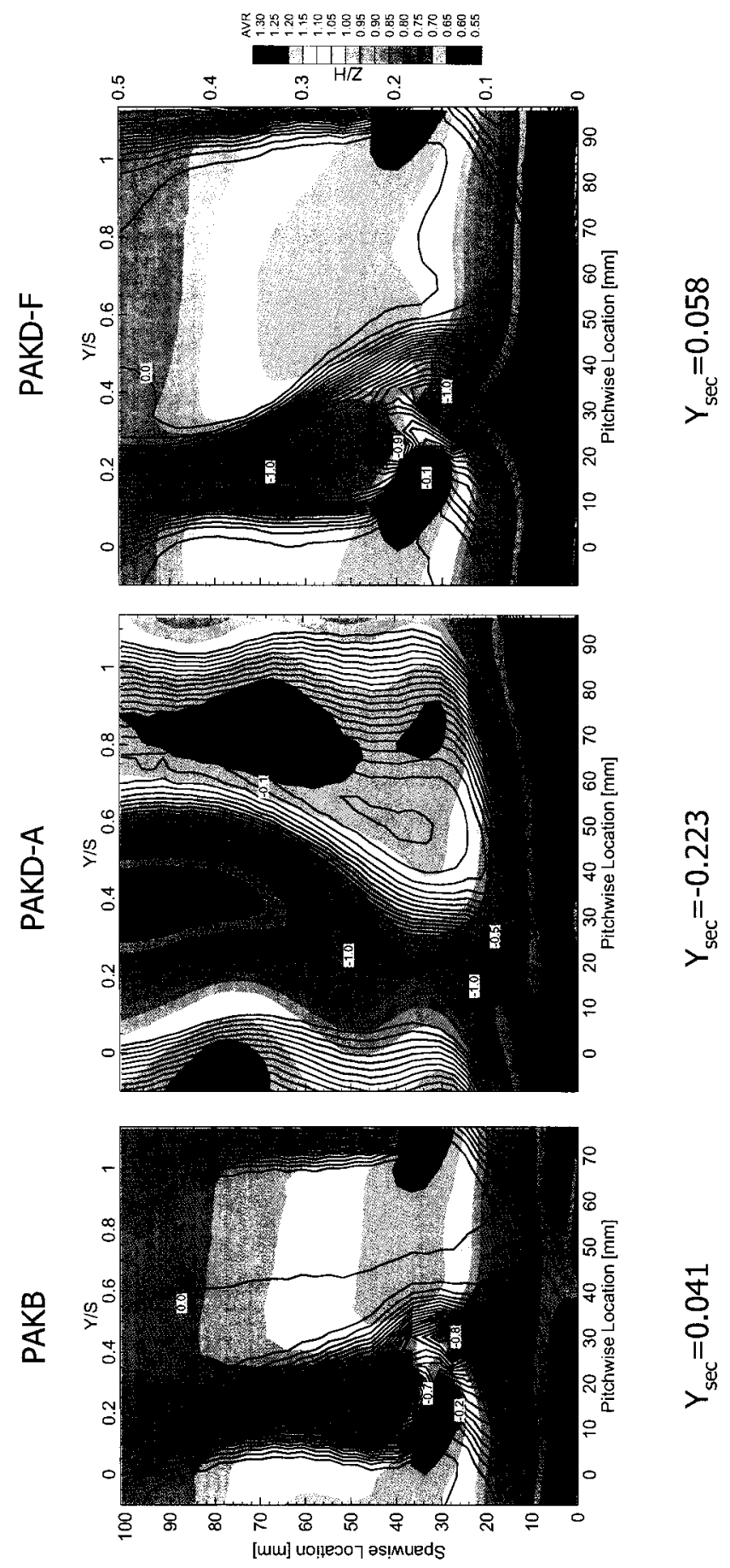

Figure I.12: Line Contours of Total Pressure Coefficient Superimposed on Flood Contours of AVR at $+5^{\circ}$ Incidence at FSTI $=1.5 \%$ 

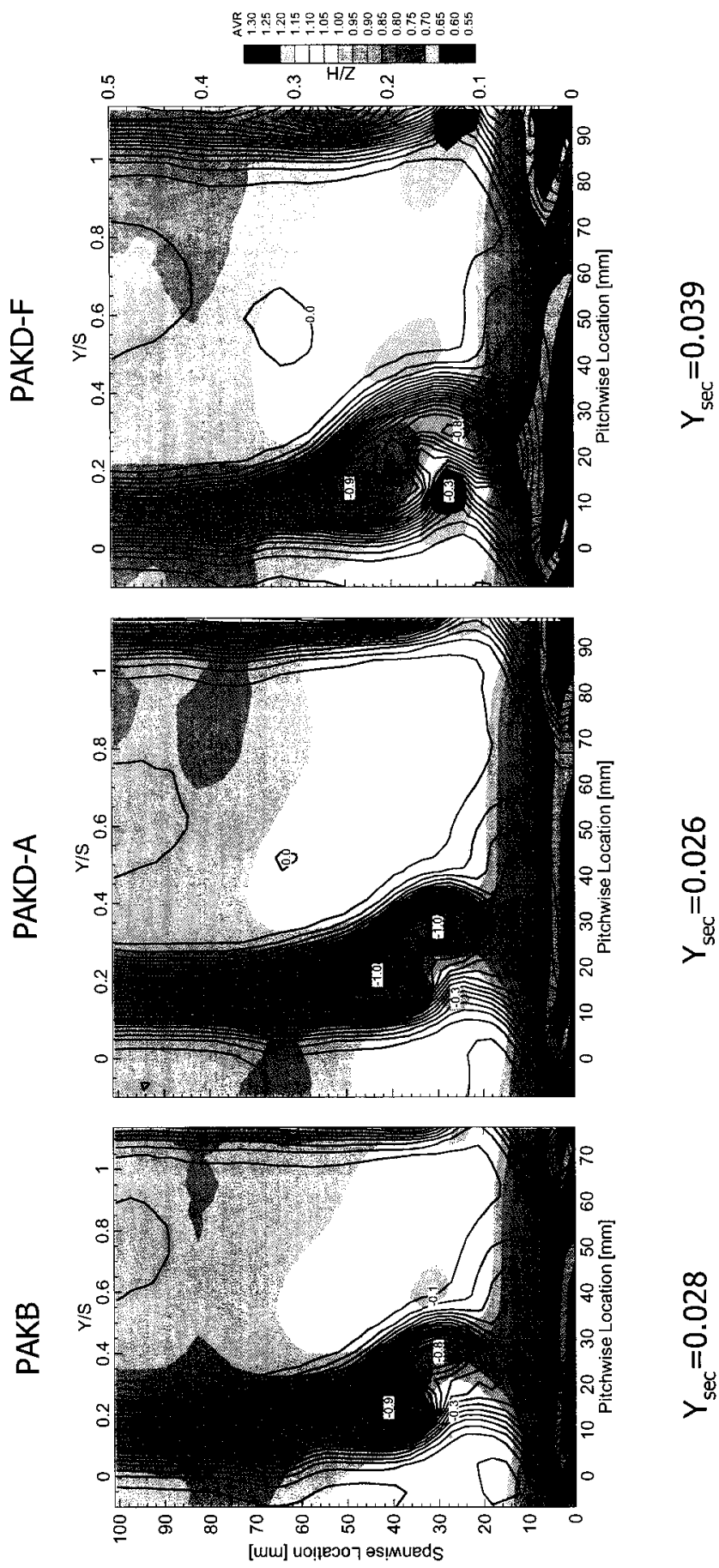

Figure I.13: Line Contours of Total Pressure Coefficient Superimposed on Flood Contours of AVR at $0^{\circ}$ Incidence at FSTI $=4.0 \%$ 

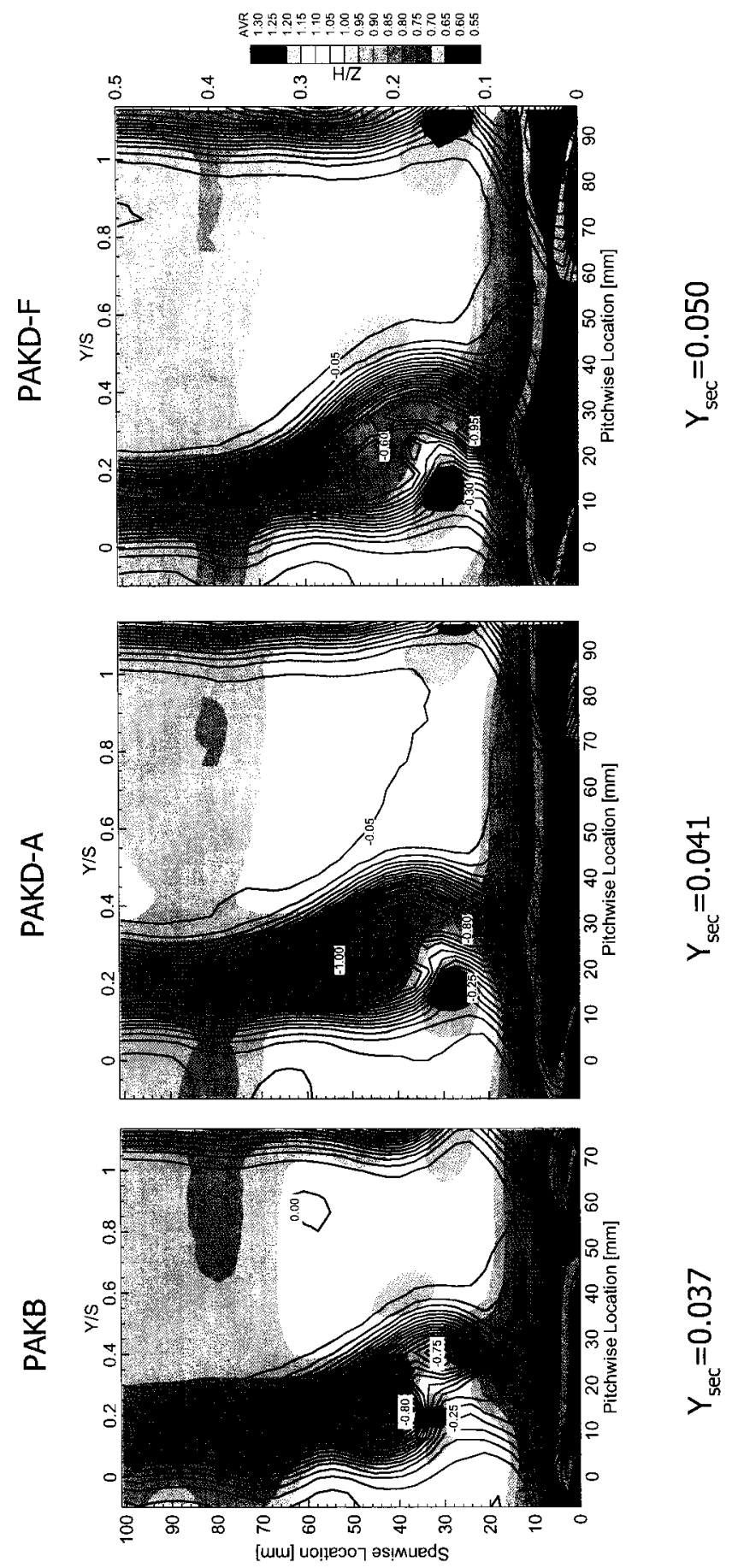

Figure 1.14: Line Contours of Total Pressure Coefficient Superimposed on Flood Contours of AVR at $+5^{\circ}$ Incidence at FSTI $=4.0 \%$ 